Schriftenreihe zum deutschen, europäischen und internationalen Wirtschaftsstrafrecht

Elisa Hoven | Michael Kubiciel

\title{
Das Gesetz gegen Doping im Sport in der Praxis - Eine Evaluierung
}

The act against doping in sport in practice An evaluation 
Schriftenreihe zum deutschen, europäischen und internationalen Wirtschaftsstrafrecht

Herausgegeben von

Prof. Dr. Dr. h.c. Uwe Hellmann, Universität Potsdam

Prof. Dr. Elisa Hoven, Universität Leipzig Prof. Dr. Dr. h.c. Michael Kubiciel, Universität Augsburg Prof. Dr. Christian Schröder, Martin-Luther-Universität Halle-Wittenberg

Band 46 
Elisa Hoven | Michael Kubiciel

\section{Das Gesetz gegen Doping im Sport in der Praxis - Eine Evaluierung}

The act against doping in sport in practice An evaluation

\section{Nomos}


Diese Publikation wurde unterstützt durch den Open-Access-Publikationsfonds der Universität Leipzig.

Die Deutsche Nationalbibliothek verzeichnet diese Publikation in der Deutschen Nationalbibliografie; detaillierte bibliografische Daten sind im Internet über http://dnb.d-nb.de abrufbar.

The Deutsche Nationalbibliothek lists this publication in the Deutsche Nationalbibliografie; detailed bibliographic data are available on the Internet at http://dnb.d-nb.de

ISBN 978-3-8487-8106-5 (Print) 978-3-7489-2524-8 (ePDF)

British Library Cataloguing-in-Publication Data

A catalogue record for this book is available from the British Library.

ISBN 978-3-8487-8106-5 (Print)

978-3-7489-2524-8 (ePDF)

Library of Congress Cataloging-in-Publication Data

Hoven, Elisa | Kubiciel, Michael

Das Gesetz gegen Doping im Sport in der Praxis - Eine Evaluierung

The act against doping in sport in practice - An evaluation

Elisa Hoven | Michael Kubiciel

309 pp.

Includes bibliographic references.

ISBN

978-3-8487-8106-5 (Print)

978-3-7489-2524-8 (ePDF)

1. Auflage 2021

() Elisa Hoven | Michael Kubiciel

Publiziert von

Nomos Verlagsgesellschaft $\mathrm{mbH} \& \mathrm{Co}$. KG

Waldseestraße 3-5 | 76530 Baden-Baden

www.nomos.de

Gesamtherstellung:

Nomos Verlagsgesellschaft mbH \& Co. KG

Waldseestraße 3-5 | 76530 Baden-Baden

ISBN 978-3-8487-8106-5 (Print)

ISBN 978-3-7489-2524-8 (ePDF)

DOI https://doi.org/10.5771/9783748925248

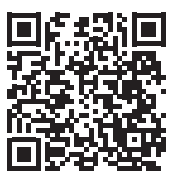

Onlineversion Nomos eLibrary

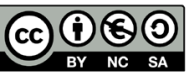

Dieses Werk ist lizenziert unter einer Creative Commons Namensnennung

- Nicht kommerziell - Weitergabe unter gleichen Bedingungen 4.0 International. 


\section{Vorwort}

Das Buch fasst die wesentlichen Erkenntnisse einer Evaluierungsstudie zur praktischen Anwendung des Anti-Doping Gesetzes zusammen und will diese sowohl dem nationalen als auch dem internationalen rechts- und sportpolitischen Dialog zur Verfügung stellen. Aus diesem Grund erscheint der Text sowohl in deutscher als auch in englischer Sprache.

Die Studie geht zurück auf einen vom Deutschen Bundestag im Jahr 2019 erteilten Beratungsauftrag im Zusammenhang mit der Evaluierung des Anti-Doping Gesetzes. Die Verfasser danken herzlich den Mitarbeiterinnen und Mitarbeitern der beteiligten Bundesministerien, zuvorderst Frau Ministerialrätin Susanne Bunke und Frau Oberregierungsrätin Sarah Thomé vom Bundesministerium der Justiz und für Verbraucherschutz sowie Frau Regierungsdirektorin Heike Mahlstedt (Bundesministerium des Inneren) und Frau Regierungsdirektorin Pia Finken (Bundesministerium für Gesundheit). Ein besonderer Dank gilt allen in den Landesjustizverwaltungen und den Staatsanwaltschaften beteiligten Stellen und Personen, die uns - trotz des damit verbundenen Aufwands und in kürzester Zeit - die relevanten Verfahrensakten übersandt oder diese in ihren Behörden zur Einsichtnahme bereitgestellt und uns vor Ort freundlich empfangen haben. Herzlich danken wir auch den Expertinnen und Experten, mit denen wir aufschlussreiche Interviews führen konnten. Zudem standen uns Strafverteidiger und Richter für weitere Hintergrundgespräche zur Verfügung - auch dafür sind wir dankbar.

Dank schulden die Verfasser nicht zuletzt jenen Personen, die sie administrativ und bei der Auswertung der Akten unterstützt haben. Zu nennen sind Frau Hannah Heuser, Herr Lennart Kretschmer, Frau Alexandra Witting (Leipzig) sowie Frau Inge Rystau und Dr. Matthias Wachter (Augsburg). Frau Sophia Hügel und Frau Annika Kubisch (Leipzig) haben sich um die Übersetzung des deutschen Textes verdient gemacht, Herr Jonathan Wehrstein (Augsburg) hat die Bibliographie erstellt. Ihnen allen sei an dieser Stelle sehr herzlich gedankt.

Während der Drucklegung des Bandes wurde bekannt, dass die Bundesregierung eine erste Empfehlung unserer Evaluierung aufgegriffen hat und die Einführung einer Kronzeugenregelung in des Anti-Doping Gesetz 
plant. Wir halten dies für einen ersten wichtigen Schritt, um das Potenzial des Gesetzes auch im Leistungssport zur vollen Entfaltung zu bringen.

\author{
Elisa Hoven \\ Michael Kubiciel
}




\section{Preface}

This book summarizes the main findings of an evaluation study on the practical application of the German Anti-Doping Act. It aims to make them available to both the national and the international legal and sports policy dialogue and is therefore published in German and English.

The study originates from an advisory mandate issued by the German Bundestag in 2019 in connection with the evaluation of the German AntiDoping Act. The authors would like to express their sincere thanks to the personnel of the participating federal ministries for their outstanding efforts - first and foremost to Ms. Susanne Bunke and Ms. Sarah Thomé (Federal Ministry of Justice and Consumer Protection) as well as Ms. Heike Mahlstedt (Federal Ministry of the Interior) and Ms. Pia Finken (Federal Ministry of Health).

Special thanks are extended to all employees involved in the state justice administrations and the public prosecutors' offices for sending us the relevant files promptly or kindly making them available for inspection in their offices, in spite of the effort involved. We would also like to thank both the experts with whom we were able to conduct informative interviews and the defense attorneys and judges, who granted us opportunities for further background discussions.

Last but not least, the authors would like to express their deepest gratitude to those who supported them administratively and in the evaluation of the files: Hannah Heuser, Lennart Kretschmer, Alexandra Witting (Leipzig), Inge Rystau and Dr. Matthias Wachter (Augsburg). Mrs. Sophia Hügel and Mrs. Annika Kubisch (Leipzig) have rendered outstanding services in the translation of the German text, and Mr. Jonathan Wehrstein (Augsburg) has prepared the bibliography. We would like to take this opportunity to thank them all very much.

While the volume was going to print, the federal government took first steps to implement one of the recommendations of our evaluation as it planned to introduce a leniency program in the Anti-Doping Act. We consider this to be an important first step in order to fully develop the potential of the law in competitive sports.

Elisa Hoven

Michael Kubiciel 


\section{Inhaltsverzeichnis/Table of Content}

Abkürzungen der Interviewpartner/Abbreviations for interview partners

Das Gesetz gegen Doping im Sport in der Praxis Eine Evaluierung

A. Grundlagen der Untersuchung 21

I. Hintergründe und Ziele der Evaluation 21

II. Methodische Umsetzung 24

B. Ergebnisse der Evaluierung 32

I. Das Verbot des Selbstdopings ( $\$ 3$ ) 32

II. Die Verbotsnormen des $₫ 2$

III. Übergreifende Aspekte 121

C. Abschließende Stellungnahme der Sachverständigen 144

I. Allgemeine Bewertung 144

II. Konkrete Empfehlungen der Sachverständigen 147

Annex: Leitfaden Staatsanwälte 157

The act against doping in sport in practice An evaluation

A. Fundamentals of the study 175

I. Background and objectives of the evaluation 175

II. Methodological implementation 178

B. Results of the evaluation 185

I. The prohibition of self-doping (section 3) 185

II. The prohibition norms of section 2 
$\begin{array}{ll}\text { III. Overarching aspects } & 267\end{array}$

$\begin{array}{ll}\text { C. Final opinion of the experts } & 287\end{array}$

$\begin{array}{ll}\text { I. General assessment } & 287\end{array}$

II. Specific recommendations of the experts 289

Annex: Guideline for public prosecutors 297

$\begin{array}{ll}\text { Literaturverzeichnis/Bibliography } & 307\end{array}$ 


\section{Abkürzungen der Interviewpartner/Abbreviations for interview partners}
A Athletenvertreter / Athletes association representative
BKA Vertreter des Bundeskriminalamts / Federal Criminal Police
Office representative
D Vertreter des Deutschen Olympischen Sportbundes / German Olympic Sports Confederation representative
$\mathrm{N} \quad$ Vertreter der NADA / NADA representative
R Richter/judge
StA Staatsanwalt / public prosecutor
Zoll Vertreter der Zollfahndung und Zollverwaltung / Customs Authorities representative 
Das Gesetz gegen Doping im Sport in der Praxis Eine Evaluierung 


\section{Inhaltsverzeichnis}

A. Grundlagen der Untersuchung 21

I. Hintergründe und Ziele der Evaluation 21

II. Methodische Umsetzung 24

1. Auswertung von Verfahrensakten 25

a) Sample 25

b) Kodierung quantitativ und qualitativ 26

c) Auswertung 27

2. Experteninterviews 28

a) Sample 28

b) Design der Interviewleitfäden 29

c) Durchführung und Auswertung der Interviews 30

B. Ergebnisse der Evaluierung 32

I. Das Verbot des Selbstdopings ( $(3)$

1. Erscheinungsformen von Selbstdoping 32

a) Betroffene Sportarten $\quad 32$

b) Angaben zu den Beschuldigten 33

c) Dopingmittel und -methoden 34

2. Materiell-rechtliche Fragen 35

a) Tathandlungen $\quad 35$

aa) Legislativer Kontext 35

bb) Quantitative Auswertung $\quad 36$

cc) Dopingmethoden 36

dd) Präzision und Nachvollziehbarkeit der

Tatbestandsformulierungen $\quad 37$

ee) Mengenunabhängige Kriminalisierung des Erwerbes
und Besitzes von Dopingmitteln

ff) Zusammenfassung 40

b) Weitere Strafbarkeitsvoraussetzungen 41

aa) Medizinische Indikation 41

(1) Legislativer Kontext 41

(2) Erkenntnisse der Studie 42 
bb) Vorteilsverschaffungsabsicht in einem Wettbewerb des organisierten Sports

(1) Auslegung des Merkmals „Wettbewerb des organisierten Sports“

(2) Absicht einer Vorteilsverschaffung

(3) Zwischenergebnis

c) Strafbarkeitseinschränkungen in $\$ 4$ Abs. 7

aa) Testpool-Athleten 48

bb) Einnahmen von erheblichem Umfang 49

(1) Teleologische Angemessenheit der Strafbarkeitseinschränkung 49

(2) Anwendungsschwierigkeiten

(3) Bestimmung und Bestimmbarkeit der Formulierung

(4) Ermittlungen

(5) Zusammenfassung 54

cc) Allgemeine Bewertung von $\$ 4$ Abs. 7

(1) Systematik 55

(2) Grund für geringe Fallzahlen und seltene Anklagen

(3) Ungleichbehandlung von Sportlern 56

d) Tätige Reue 58

aa) Legislativer Kontext 58

bb) Ergebnisse der Studie $\quad 59$

3. Erkenntnisse zu prozessualen Fragen 59

a) Kenntniserlangung $\quad 59$

aa) Wege der Kenntniserlangung 59

bb) Kronzeugenregelung $\quad 61$

b) Ermittlungsmaßnahmen $\quad 62$

c) Abschluss der Verfahren 63

aa) Keine Verurteilungen wegen Selbstdopings 65

bb) Einstellungen nach $₫ 170$ Abs. 2 StPO oder $₫ 153$ StPO 65

cc) Einstellung wegen eines fehlenden Vorsatznachweises bezüglich der Einnahme von Dopingmitteln $\quad 67$

dd) Einstellung ohne erkennbare Gründe $\quad 71$

d) Strafbefehle und Strafzumessung $\quad 73$

4. Bewertung 74

a) Allgemeine Bewertung der strafrechtlichen Regelung von Selbstdoping

aa) Überblick 
bb) Zur Angemessenheit der Kriminalisierung des

Selbstdopings

cc) Zur Angst vor vorsätzlichen Falschbeschuldigungen $\quad 77$

dd) Zur Sorge um die Bedeutung der Sportgerichtsbarkeit $\quad 77$

ee) Generelle Bewertung 78

b) Vorschläge aus der Praxis zur Reform der strafrechtlichen Regelungen zum Selbstdoping

aa) Einführung einer speziellen Kronzeugenregelung $\quad 79$

bb) Verbesserter Schutz von Hinweisgebern 82

II. Die Verbotsnormen des $₫ 2$

1. Phänomen 84

a) Erkenntnisse zu Tätern und Tathandlungen 84

b) Erkenntnisse zu Dopingmitteln $\quad 87$

c) Zusammenfassung $\quad 88$

2. Feststellungen zu materiell-rechtlichen Aspekten von $₫ 2$

a) Die Tatbestände und ihre Voraussetzungen 88

aa) Legislativer Kontext $\quad 88$

bb) Herstellung, Handeltreiben, Inverkehrbringen, Verschreiben ( $\$ 2$ Abs. 1) 91

cc) Anwendung von Dopingmitteln und -methoden ( $\$ 2$ Abs. 2)

dd) Erwerbs-, Besitz- und Durchverbringung nach $₫ 2$ Abs. 3

(1) Allgemeine Bedeutung 94

(2) Erwerb und Besitz nicht geringer Mengen 94

(3) Verbringen in den Geltungsbereich und durch den Geltungsbereich

ee) Zum Zweck des Dopings beim Menschen im Sport $\quad 99$

ff) Fahrlässigkeitsstrafbarkeit nach $\$ 4$ Abs. $6 \quad 101$

b) Qualifikationstatbestände 103

c) Zusammenfassung 105

3. Erkenntnisse zu prozessualen Fragen 105

a) Anfangsverdacht 106

b) Ermittlungsmaßnahmen 107

c) Abschluss der Verfahren 111

d) Besonderheiten des Gerichtsverfahrens 112

4. Bewertung 114

a) Allgemeine Bewertung 114

b) Gesetzgeberische Umsetzung 116 
c) Verbesserungsvorschläge 118

aa) Materielles Recht 118

bb) Prozessrecht und Ermittlungsbefugnisse 119

III. Übergreifende Aspekte 121

1. Strafrahmen und Verjährung 121

a) Strafrahmen in $\$ 4 \quad 121$

aa) Anhebung der Höchststrafe in $\mathbb{4} 4$ Abs. 1

bb) Inkonsistenz der geltenden Strafrahmen? 124

b) Verjährung 125

c) Bewertung 126

aa) Höchststrafe 126

bb) Erwerbs- und Besitzstrafbarkeit 127

cc) Verjährungsregelungen 127

2. Einrichtung von Schwerpunktstaatsanwaltschaften und -gerichten 128

a) Die Forderung von NADA und Athletenverbänden 128

b) Zur Einrichtung von Schwerpunktstaatsanwaltschaften 129

aa) Die Sicht der befragten Experten aus der Justiz 129

bb) Erkenntnisse aus der Aktenauswertung 133

c) Zur Einrichtung von Schwerpunktgerichten 133

d) Bewertung 136

3. Zusammenarbeit mit der NADA und $\$ 8$ AntiDopG 138

4. Schulungen 141

C. Abschließende Stellungnahme der Sachverständigen 144

I. Allgemeine Bewertung 144

1. Selbstdoping $(\$ 3)$

2. Unerlaubter Umgang mit Dopingmitteln und unerlaubte Anwendung von Dopingmethoden $(\$ 2)$

II. Konkrete Empfehlungen der Sachverständigen 147

1. Materiell-rechtliche Änderungen 147

a) Selbstdoping 147

b) Unerlaubter Umgang mit Dopingmitteln $(\$ 2) \quad 152$

2. Allgemeine prozessuale und institutionelle Änderungen 155

a) Kronzeugenregelung und Hinweisgebersysteme 155

b) Schwerpunktstaatsanwaltschaften und
Schwerpunktgerichte

Annex: Leitfaden Staatsanwälte 157 


\section{Hinweise}

1. Sämtliche Paragraphen ohne nähere Bezeichnung verweisen auf das Gesetz gegen Doping im Sport (AntiDopG).

2. Der gelegentliche Gebrauch des generischen Maskulinums ist der Lesbarkeit der Studie geschuldet und schließt Personen aller Geschlechter ein. 


\section{A. Grundlagen der Untersuchung}

\section{Hintergründe und Ziele der Evaluation}

Das Gesetz zur Bekämpfung von Doping im Sport (AntiDopG) vom 10.12.2015 soll die Integrität des Sports, die Fairness und Chancengleichheit im Wettbewerb sowie die Gesundheit der Sportler schützen, indem es den Einsatz von Dopingmitteln und Dopingmethoden sowie verschiedene Formen der Vorbereitung unter Strafe stellt (vgl. $\$ 1$ AntiDopG ${ }^{1}$ ). Erwartet wird vom AntiDopG ein „negativer Gegenanreiz“, um die „Dopingaffinität“, die mit der wachsenden Kommerzialisierung des Sports einhergeht, auszugleichen. ${ }^{2}$ Neben diesem Abschreckungseffekt wird den strafbewehrten Verboten des AntiDopG auch eine generalpräventive Wirkung zugeschrieben: Sie sollen dazu beitragen, das Verbot des Dopings, insbesondere des Selbstdopings, stärker und tiefer als bisher sozialethisch zu verankern. Damit sollen die Bemühungen der Sportverbände unterstützt werden, denen - anders als dem Staat - wichtige Mittel zur Durchsetzung des Doping-Verbots fehlen. ${ }^{3}$

Die bis zum Dezember 2015 geltenden Regeln hatten sich nach Auffassung des Gesetzgebers als nicht ausreichend erwiesen, da die seinerzeit existierenden dopingspezifischen Strafvorschriften insbesondere mit Blick auf Vorbereitungshandlungen, den Besitz geringer Mengen von Dopingmitteln zur Eigen-Applikation und - vor allem - in Bezug auf das sog. Selbstdoping Lücken aufwiesen. ${ }^{4}$ Das AntiDopG hat daher nicht nur die bereits bestehenden Regelungen des AMG übernommen und ihnen damit eine neue - u.U. generalpräventiv nützliche - Sichtbarkeit verliehen. Das Recht ist auch um weitere Verbote bzw. Straftatbestandsvarianten ergänzt worden, insbesondere um das strafbewehrte Verbot des Selbstdopings in Wettbewerben des organisierten Sports, neue Vorfeldtatbestände sowie die Erweiterung von Qualifikationstatbeständen mit daran anknüpfenden weiterreichenden Ermittlungsbefugnissen. Das AntiDopG hat damit den Kreis des verbotenen Verhaltens sowie die strafprozessualen Möglichkeiten zur

1 Im Folgenden sind alle nicht näher bezeichneten Normen solche des AntDopG.

2 Siehe dazu Rössner, in: Lehner/Nolte/Putzke, AntiDopG, 2017, Vor $\$ 1$ Rn. 30.

3 Dazu schon Steiner, NJW 1991, 2729, 2733.

4 BT-Drs. 18/4898, S. 2. 
Durchsetzung des Rechts erweitert. Darüber hinaus hat es auch das funktional-institutionelle Arrangement verändert: Das betrifft insbesondere den strukturierten Informationsaustausch zwischen der Nationalen Anti Doping Agentur (NADA) und den Staatsanwaltschaften. Hinzu treten informelle Wirkungen: Drohen Sportlerinnen, Trainern und anderen im organisierten Sport tätigen Personen neue strafrechtliche Risiken, dürften auch die Verbände (und Vereine) ihre Präventionsmaßnahmen angepasst und damit dem Gesetz zusätzliche präventive Wirkung verliehen haben.

Fast fünf Jahre nach Inkrafttreten des Gesetzes stellt sich die Frage, wie die Praxis die veränderten Möglichkeiten nutzt, und ob das AntiDopG der Erreichung der genannten Ziele nähergekommen ist.

In der Rechtswissenschaft finden sich dazu einander widersprechende Aussagen. Während sich manche angesichts der vergleichsweise geringen Anzahl abgeschlossener Strafverfahren dezidiert "pessimistisch“ zeigen, ${ }^{5}$ verweisen andere auf die präventive Wirkung, die sich nicht an Verfahrenszahlen ablesen lasse. ${ }^{6}$ Von Athletinnen und Athleten hört man gelegentlich die Sorge, die Weite der Straftatbestände könne zur Einleitung eingriffsintensiver Ermittlungsverfahren führen, die sich letztlich als gegenstandslos erweisen. ${ }^{7}$ Ermittler halten dem entgegen, das Gesetz ermögliche zielgerichtete Ermittlungen; die anfänglich geringe Anzahl von Strafverfahren sei nicht ungewöhnlich, sondern liege an der Neuheit der Rechtsmaterie und der Art der Verfahren. ${ }^{8}$

Die Studie dient als Grundlage für die in Art. 8 des Gesetzes zur Bekämpfung von Doping im Sport vorgeschriebene Wirkungsevaluation des neuen AntiDopG. Sie soll die Ermittlung und Bewertung der Auswirkungen der Gesetzesänderungen auf die strafrechtliche Ahndung von Dopingverstößen wissenschaftlich unterstützen und dabei mögliche Schwachstellen identifizieren, die bei der Gesetzesanwendung ersichtlich werden, um Verbesserungsmaßnahmen zu ermöglichen. Leitend für die Studie ist daher die Frage, ob und wie sich die vom AntiDopG herbeigeführten Rechtsänderungen auf die Rechtspraxis ausgewirkt haben. Neben der Untersuchung der strafrechtlichen Verfolgungspraxis soll dabei auch die Wahrnehmung der gesetzlichen Änderungen durch Vertreterinnen und Vertreter

5 So Jahn, in: Hoven/Kubiciel, Korruption im Sport, 2018, S. 117, 123 ff.; skeptisch bereits Kreuzer, ZRP 2013, 181, $182 \mathrm{f}$.

6 So Rössner, zitiert nach Armenat, in: Hoven/Kubiciel (Fn. 5) S. 135 ff., 139 ff.

7 Kassner, in: Hoven/Kubiciel (Fn. 5), S. 135 ff.

8 Nachweise im Diskussionsbericht von Armenat, in: Hoven/Kubiciel (Fn. 5), S. 139, 144. 
externer Organisationen wie der NADA oder den Athletenverbänden beleuchtet werden.

Die zentralen Leitfragen der Evaluation lauteten:

I. Phänomen

- Welche Formen von Doping sind in der Praxis der Strafverfolgung relevant (Sportart, Täterperson, Art des Dopings etc.)?

II. Strafverfolgung von Selbstdoping

- Wie sieht die Strafverfolgung von Selbstdoping in der Praxis aus?

o Wie wird ein Anfangsverdacht generiert?

o Welche Ermittlungsmaßnahmen werden durchgeführt?

o Wegen welcher Tathandlungen wird ermittelt und ggf. angeklagt und verurteilt?

o Wie lange dauern die Verfahren?

o Wie lassen sich die Beschuldigten im Verfahren ein?

o Wie werden Ermittlungsverfahren abgeschlossen - und mit welcher Begründung?

o Welche Beweisprobleme stellen sich im Ermittlungsverfahren und vor Gericht?

- Wie werden die subjektiven Tatvoraussetzungen nachgewiesen, also etwa die Vorteilsverschaffungsabsicht in $₫ 3$ Abs. 1, 2 oder das Wissen um fehlende Indikation in $\$ 3$ Abs. 2, 4?

- Wie ließ sich belegen, dass ein Dopingmittel bzw. eine Dopingmethode „ohne medizinische Indikation“ angewendet worden ist?

- Welche zusätzlichen strafprozessualen Möglichkeiten werden für sinnvoll erachtet? (Bsp.: Kronzeugenregelung)

o Welche rechtlichen Fragen stellen sich bei der Anwendung des AntiDopG?

- Wie wird bestimmt, ob ein Wettbewerb des organisierten Sports vorliegt?

- Wie wird der durch $₫ 4$ Abs. 7 beschriebene Adressatenkreis verstanden?

- Wie werden die "Einnahmen von erheblichem Umfang" in $\$ 4$ Abs. 7 Nr. 2 bestimmt?

o Gibt es Fälle tätiger Reue nach $₫ 4$ Abs. 8?

o Welche Sanktionen wurden verhängt?

III. Strafverfolgung von $\$ 2$ i.V.m. $\$ 4$

- Wie sieht die Strafverfolgung von $₫ 2$ in der Praxis aus?

o Wie wird ein Anfangsverdacht generiert?

o Welche Ermittlungsmaßnahmen werden durchgeführt? 
o Welche Änderungen haben sich durch die Einführung von $₫ 2$ für die Praxis ergeben?

- Spielt die Erweiterung des Tatbestandes um zusätzliche Tathandlungen eine Rolle?

- Spielt es eine Rolle, dass das Gesetz nunmehr auch die unerlaubte Anwendung von Dopingmethoden erfasst?

- Wie wichtig und wie praktikabel sind die Fahrlässigkeitstatbestände $\$ 4$ Abs. 6 ?

- Wie wirkt sich die Einschränkung der Besitzstrafbarkeit nach $\$ 2$ Abs. 3 auf Fälle „nicht geringer Mengen“ in der Praxis aus?

- Hat die Einführung von Qualifikationstatbeständen (anstelle von besonders schweren Fällen) in der Praxis eine Änderung bewirkt?

- Hat sich in der Praxis etwas dadurch geändert, dass die Taten jetzt mögliche Vortaten für die Geldwäsche sind?

o Wie werden die Verfahren abgeschlossen?

IV. Allgemeine Fragen und Einschätzungen

- Wie werden Strafrahmen und Verjährungsfristen beurteilt?

- Wie wird die Einsetzung von Schwerpunktstaatsanwaltschaften und -gerichten bewertet?

- Welche Bedeutung hat die Kooperation mit der NADA nach $\$ 8$ ?

- Wie bewerten PraktikerInnen die strafrechtlichen und strafprozessualen Änderungen im AntiDopG insgesamt?

\section{Methodische Umsetzung}

Bei der Studie handelt es sich um eine reine Hellfelduntersuchung. Da Ziel der Studie nicht die Erforschung der tatsächlichen Verbreitung von Doping, sondern allein die Erfassung der Auswirkungen des AntiDopG auf die strafrechtliche Ahndung von Dopingverstößen ist, spielt das Dunkelfeld für die vorliegende Untersuchung keine entscheidende Rolle.

Folgende Methoden kamen zum Einsatz:

- Qualitative und quantitative Auswertung sämtlicher Verfahrensakten zum Selbstdoping

- Experteninterviews zu praktischen Erfahrungen mit dem AntiDopG 
1. Auswertung von Verfahrensakten

a) Sample

Die Aktenauswertung beschränkt sich nach den Vorgaben des Bundesministeriums der Justiz und für Verbraucherschutz (BMJV) auf Verfahren zum Selbstdoping nach $\$ 3$. Ziel war eine Totalerhebung aller thematisch einschlägigen Akten von Ermittlungsverfahren aus nicht-laufenden Verfahren seit Einführung der Strafbarkeit von Selbstdoping. Hierzu wurden zunächst von Seiten des BMJV die Landesjustizministerien um Auskunft über einschlägige Verfahren in den verschiedenen Staatsanwaltschaften ihres Bundeslandes gebeten. Anschließend kontaktierten die Sachverständigen erneut sämtliche Landesjustizministerien sowie die gemeldeten Staatsanwaltschaften und stellten Antrag auf Akteneinsicht nach $\$ 476$ StPO. Alle Staatsanwaltschaften stellten die bei ihnen vorhandenen Akten zur Verfügung. Nicht alle den Sachverständigen zur Verfügung gestellten Akten betrafen den Untersuchungsgegenstand; vielmehr wurden auch Akten zu Verfahren übermittelt, die ausschließlich wegen Verstößen gegen \2 AntiDopG geführt wurden.

Zur Verfügung gestellt wurden insgesamt 526 Akten. Davon hatten tatsächlich 103 Verfahren Selbstdoping zum Gegenstand. Erfasst wurden dabei sowohl Verfahren, die ausdrücklich nach $\$ \mathbb{S} 3,4$ geführt wurden als auch Verfahren, in denen keine explizite Nennung des Tatbestandes erfolgte, bei denen im Rahmen der Ermittlungen allerdings erkennbar die Voraussetzungen des Selbstdopings geprüft wurden. Nur diese Verfahren gingen in die Auswertung ein. Die Akten verteilen sich wie folgt auf die verschiedenen Staatsanwaltschaften.

\begin{tabular}{|l|l|l|}
\hline Bundesland & Zur Verfügung gestellt & Einschlägig \\
\hline Baden-Württemberg & 35 & 35 \\
\hline Bayern & 27 & 7 \\
\hline Berlin & 9 & 0 \\
\hline Brandenburg & 19 & 3 \\
\hline Bremen & 5 & 1 \\
\hline Hamburg & 1 & 1 \\
\hline Hessen & 12 & 8 \\
\hline Mecklenburg-Vorpommern & 21 & 4 \\
\hline
\end{tabular}




\begin{tabular}{|l|l|l|}
\hline Bundesland & Zur Verfügung gestellt & Einschlägig \\
\hline Niedersachsen & 39 & 7 \\
\hline Nordrhein-Westfalen & 248 & 29 \\
\hline Rheinland-Pfalz & 7 & 1 \\
\hline Saarland & 1 & 1 \\
\hline Sachsen & 71 & 0 \\
\hline Sachsen-Anhalt & 5 & 2 \\
\hline Schleswig-Holstein & 12 & 1 \\
\hline Thüringen & 14 & 3 \\
\hline Gesamt & $\mathbf{5 2 6}$ & $\mathbf{1 0 3}$ \\
\hline
\end{tabular}

Die geringen Fallzahlen entsprechen auch der Einschätzung der Staatsanwaltschaften:

„Es hat bei uns die Anwendung des $\$ 3$ noch so gut wie keine Rolle gespielt, zumindest in meiner Tätigkeit. Da wüsste ich nicht, dass ich irgendwo mal einen Beschuldigten nach \$3 gehabt hätte." (StA 5)

b) Kodierung quantitativ und qualitativ

Die Einsichtnahme in die Akten erfolgte teilweise in den Räumen der Staatsanwaltschaften, teilweise wurden die Akten an die Universitäten Augsburg oder Leipzig versendet. Für die Kodierung der Akten wurde - in Abstimmung mit den beteiligten Ministerien - ein qualitativer Kodierbogen (in Excel) entwickelt, der 32 Kategorien zu Phänomen, Ermittlungsund Gerichtsverfahren sowie den aufgeworfenen Rechts- und Beweisfragen enthielt. Die Festlegung der Kategorien folgte den zuvor erarbeiteten Forschungsfragen, zu denen detaillierte Unterfragen formuliert wurden. Um durch die getroffenen Vorannahmen keine für die Evaluation relevanten Aspekte auszuklammern, wurde eine offene Kategorie („Sonstige Besonderheiten") aufgenommen.

Für die quantitative Auswertung wurde ein Codebuch angelegt, in dem numerisch zu erfassende Daten - etwa die Häufigkeit der betroffenen Sportarten oder die Form des Verfahrensabschlusses - festgehalten wurden.

Um eine größtmögliche Übereinstimmung in der Kodierung zu gewährleisten, wurden die ersten Verfahrensakten von den Sachverständigen ge- 
meinsam kodiert. Anschließend wurden die Analyseergebnisse mit Blick auf etwaige Abweichungen verglichen. Auf diese Weise konnten frühzeitig Unterschiede in der Kodierung offengelegt und Divergenzen behoben werden. Erst nachdem eine Kodierübereinstimmung von über 90 Prozent erreicht wurde, erfolgte eine selbständige Übertragung der Akten in den Analysebogen. ${ }^{9}$

\section{c) Auswertung}

Die Erkenntnisse der qualitativen Aktenanalyse wurden in eine gemeinsame Excel-Tabelle übertragen. Die Tabelle konnte anschließend vertikal und horizontal ausgewertet werden. Die vertikale Betrachtung ermöglichte eine verfahrensübergreifende Analyse bestimmter Merkmale und Kategorien; die horizontale Lesart zeichnete das vollständige Bild eines konkreten Verfahrens.

Die Auswertung der quantitativen Daten erfolgte mittels des Statistikprogrammes IBM SPSS Statistics 26. Zunächst wurden die Ergebnisse der qualitativen Datenerhebung entsprechend der Vorgaben aus dem Codebuch in einer separaten Excel-Tabelle kodiert. Der Datensatz wurde anschließend in SPSS importiert und auf seine Vollständigkeit verifiziert. Die Kodierung der Informationen erfolgte weitestgehend in einer nominalen Skalierung. Mehrfachnennungen waren in Einzelfällen möglich (z.B. im Rahmen der angewendeten Zwangsmaßnahmen). Für die Bestimmung von Zeitabständen (z.B. der Dauer des Ermittlungsverfahrens) wurde eine metrische Skalierung gewählt.

Nach der Datenaufbereitung wurde der vollständige Datensatz ( $\mathrm{n}=103$ ) mittels deskriptiver Statistik verdichtet, um Messzahlen sowie Tabellen und Grafiken zu den oben benannten Forschungsfragen zu liefern. ${ }^{10} \mathrm{Da}$ die vorliegenden Ergebnisse auf einer Vollerhebung aller in Deutschland durchgeführten Ermittlungsverfahren wegen des Anfangsverdachts auf Selbstdoping beruhen, ist - vorbehaltlich der Zusendung aller einschlägi-

9 Nach Rössler/Geise gilt für inhaltliche Kategorien zu komplexen Konstrukten eine Übereinstimmung mit 80 Prozent als Mindeststandard; siehe Rössler/Geise, in: Möhring/Schlütz (Hrsg.), Handbuch standardisierte Erhebungsverfahren in der Kommunikationswissenschaft, 2013, S. 269.

10 Vgl. zum methodischen Vorgehen Kosfeld/Eckey/Türck (Hrsg.), Deskriptive Statistik, 6. Aufl., 2016, S. 37 ff. 
gen Verfahrensakten - eine Prognose durch induktive Statistik nicht erforderlich.

Die nominal skalierten Daten wurden auf ihre absolute sowie relative Häufigkeitsverteilung untersucht. Dabei wurden die Messzahlen im Hinblick auf ihre relative Häufigkeit sowohl gegen den gesamten Datensatz $(\mathrm{n}=103)$ als auch gegen die Anzahl aller einschlägigen Verfahren ( $\mathrm{n}=$ variabel) gerechnet. Zur besseren Veranschaulichung wurden die Messzahlen zusätzlich durch Kreis- oder Balkendiagramme visualisiert. Die metrisch skalierten Daten sind im Hinblick auf Mittel- sowie Minimal- und Maximalwert ausgewertet worden. Die Ausweisung des Medians war aufgrund der geringen Schwankungen nicht erforderlich.

\section{Experteninterviews}

Die Durchführung von Experteninterviews diente zum einen der Evaluation von $\$ 2$, der nicht Gegenstand der Aktenuntersuchung war. Zum anderen konnten die Gespräche die Aktenauswertung zum Selbstdoping in wichtigen Punkten ergänzen: Erfahrungsgemäß sind Strafakten in ihrem Informationsgehalt lückenhaft und nehmen entscheidende Strategien und Hintergründe, wie Absprachen, faktische Hindernisse und Verteidigungsstrategien, nur unzureichend auf.

a) Sample

Interviews wurden mit folgenden Experten ${ }^{11}$ geführt:

- 1 Vertreter der NADA

- 1 Vertreter Zollfahndungsamt Frankfurt a.M.

- 1 Vertreter Hauptzollamt Frankfurt a.M.

- 1 Vertreter Zollkriminalamt

- 1 Vertreter BKA

- 1 Vertreter Neue Athletenvertretung

- 1 Vertreter Athletenvertretung bei DOSB

- 1 Vertreter DOSB

- 2 Staatsanwälte StA Frankfurt aM

11 Für die Experten wird das generische Maskulinum verwendet, um keine Rückschlüsse auf die Person des Befragten zu erlauben; erfasst sind Personen jeden Geschlechts. 
- 2 Staatsanwälte SchwerpunktStA Zweibrücken

- 2 Staatsanwälte SchwerpunktStA Freiburg

- 1 Staatsanwalt SchwerpunktStA München ${ }^{12}$

- 1 Richter AG Zweibrücken ${ }^{13}$

- 1 Richter LG München

Die konkrete Auswahl einzelner Interviewpersonen geschah größtenteils durch Anfrage bei den Behörden bzw. Vereinigungen. Die Gefahr einer strategischen Selektion durch die auswählende Mittelsperson ${ }^{14}$ erschien hier gering, da sich die Benennung der Experten weitgehend aus den Verantwortungsbereichen innerhalb der jeweiligen Institution ergab. Tatsächlich ist in keinem Fall der Verdacht einer strategischen Auswahl entstanden. Nach dem Eindruck der Sachverständigen haben die Mittelspersonen jene Experten benannt, die in ihrem Zuständigkeitsbereich über die größte Erfahrung mit der Doping-Bekämpfung verfügten.

b) Design der Interviewleitfäden

Um Antworten auf alle relevanten Forschungsfragen zu erhalten, wurden die Interviews durch Gesprächsleitfäden strukturiert, die die Sachverständigen in Abstimmung mit den beteiligten Ministerien erarbeitet haben. Für jede zu befragende Gruppe wurden eigene Leitfäden entwickelt, die allerdings, um eine Vergleichbarkeit der Ergebnisse zu gewährleisten, im Kern dieselben Fragen enthielten, aber jeweils auf den konkreten Erfahrungsbereich der Interviewperson zugeschnitten waren.

In den Experteninterviews war eine stärkere Steuerungs- und Strukturierungsfunktion unschädlich, da weniger die subjektiven Relevanzsysteme der Befragten als vielmehr fachspezifische Fragestellungen im Vordergrund standen. ${ }^{15}$ Die Orientierung an einem Leitfaden gewährleistete eine

12 Ein geplantes zweites Interview kam wegen Terminschwierigkeiten, unter anderem in Folge der Corona-Pandemie, nicht mehr zustande.

13 Abgesehen wurde von Interviews mit Richtern am LG Zweibrücken sowie einem weiteren Richter am AG Zweibrücken, da nach Auskunft der dortigen Justiz bislang lediglich ein Richter am AG Zweibrücken über nennenswerte Erfahrungen mit dem AntiDopG verfügt.

14 Kruse, Einführung in die Qualitative Interviewforschung, 2011, S. 93.

15 Kruse, Einführung in die Qualitative Interviewforschung, 2011, S. 70. Zur Konstruktion von Leitfäden bei Experteninterviews: Bogner/Littig/Menz, Interviews mit Experten, 2014, S. 27 f. 
Vergleichbarkeit der Interviews im Rahmen der späteren Analyse. ${ }^{16}$ Um trotz des vergleichsweise hohen Strukturierungsniveaus dem qualitativen Prinzip der Offenheit Rechnung zu tragen, ${ }^{17}$ enthielt der Leitfaden zunächst thematisch fokussierte, aber narrativ offene Erzählaufforderungen, die durch mögliche detailliertere Nachfragen ergänzt werden konnten. ${ }^{18}$

Beispiel für eine offene Fragestellung:

„Welche Ermittlungs- und Zwangsmaßnahmen setzen Sie für die Aufklärung von Doping-Fällen ein und mit welchem Erfolg?"

Beispiel für eine mögliche Nachfrage:

„Hat die Zulässigkeit der TKÜ Ihre Ermittlungsmöglichkeiten verbessert?“"

Die Übersetzung der allgemeinen Forschungsfragen in konkrete und für die Experten nachvollziehbare Interviewfragen ${ }^{19}$ erfolgte im Wege des von Helfferich entwickelten methodisierten „SPSS“-Verfahrens..$^{20}$ Der Leitfaden für die Interviews mit den Experten enthielt verschiedene modulare Inhaltsbereiche zu Phänomen und Verfolgung von Doping. ${ }^{21}$ Ein exemplarischer Leitfaden ist als Anlage beigefügt.

\section{c) Durchführung und Auswertung der Interviews}

Die Interviews wurden zum größten Teil telefonisch, zum kleineren Teil in persönlicher Anwesenheit geführt und mit Zustimmung der Interviewpersonen aufgezeichnet. Die Aufnahmen der im Schnitt etwa 30-40 Minuten dauernden Gespräche wurden anschließend für die Auswertung transkribiert. Die Auswertung der Interviews erfolgte rein qualitativ und nach denselben Grundsätzen wie die Analyse der Akten. Die Leitfäden wurden

16 Helfferich, in: Baur/Blasius (Hrsg.), Handbuch Methoden der empirischen Sozialforschung, 4. Aufl. 2014, S. 559, 566.

17 Hoffmann-Riem, Kölner Zeitschrift für Soziologie und Sozialpsychologie 1980, 325; Kruse, in: Gredig/Schnurr (Hrsg.), Forschen in der Sozialen Arbeit, 2011, S. 158.

18 Damit wurde im Design der Leitfäden das von Helfferich formulierte Prinzip „So offen wie möglich, so strukturierend wie nötig" umgesetzt; Helfferich, in: Baur/ Blasius, Handbuch Methoden der empirischen Sozialforschung, 4. Aufl. 2014, S. 559,560 .

19 Kaiser, Qualitative Experteninterviews, 2014, S. 52.

20 Helfferich, Qualität qualitativer Daten, 2005.

21 Exemplarisch findet sich der Leitfaden für die Interviews mit den Staatsanwaltschaften im Annex der Arbeit. 
in Kategorien übersetzt und die Antworten in eine gemeinsame Excel-Tabelle übertragen. 


\section{B. Ergebnisse der Evaluierung}

\section{Das Verbot des Selbstdopings (\$3)}

1. Erscheinungsformen von Selbstdoping

Die einschlägigen Verfahren weisen die nachfolgend skizzierten phänomenologischen Merkmale auf.

\section{a) Betroffene Sportarten}

Die untersuchten Verfahren betrafen insbesondere den Bereich des Kraftsports $\left(20,4 \%{ }^{22}\right)$ und des Bodybuildings $(18,4 \%) .{ }^{23}$ Die übrigen Verfahren verteilten sich auf Sportarten wie den Kampfsport (13,6\%), den Radsport (4,9\%), die Leichtathletik (5,8\%) oder den Marathon (3,9\%).

22 Prozentangaben werden auf eine Stelle hinter dem Komma gerundet.

23 Dem Kraftsport zugerechnet werden u.a. der Kraftdreikampf, das Bankdrücken und Gewichtheben, während beim Bodybuilding die Präsentation des Körpers und nicht die Erbringung von Kraftleistungen im Fokus steht. 
Abb. 1: Verteilung der Sportarten im untersuchten Sample



Kraftsport Bodybuilding Kampfsport $\square$ Leichtathletik Marathon Bradsport $\square$ Eisschnellauf $\square$ Sonstiges Nkeine Angabe

Sonstige von der NADA unter der Risikogruppe A erfasste Sportarten ${ }^{24}$ wie Eisschnelllauf, Kanusport, Schwimmen, Triathlon oder Rudern finden sich im Sample nicht oder nur in jeweils einem einzelnen Verfahren.

b) Angaben zu den Beschuldigten

Die Beschuldigten waren mehrheitlich männlich $(86,1 \%)$ und wurden überwiegend in den neunziger $(37,9 \%)$ und achtziger $(28,2 \%)$ Jahren geboren. Unter den Beschuldigten waren zwei Jugendliche und 14 Heranwachsende. Lediglich ein Verfahren wurde wegen einer Teilnahme am Selbstdoping geführt; in den übrigen 102 Verfahren wurde den Beschuldigten eine täterschaftliche Begehung vorgeworfen.

24 https://www.nada.de/fileadmin/user_upload/nada/DKS/160729_UEbersicht_Risi kogruppen.pdf (letzter Abruf: 26. Mai 2020). 
c) Dopingmittel und -methoden

In 96,1\% der Verfahren bezogen sich die Ermittlungen ausschließlich auf verbotene Dopingmittel, in 2,9\% der Verfahren auf Dopingmethoden und in $1 \%$ auf beides. Die vergleichsweise geringe Bedeutung der Dopingmethoden bestätigte sich auch in den Interviews.

Fragesteller: „Es sind gesetzlich auch Dopingmethoden erfasst, nicht nur Dopingmittel. Hatte das für Ihre Arbeit schon mal eine Bedeutung?"

StA 5: "Nein. Tatsächlich in der Tat keine. Ich habe zwar jetzt auch zwei Verfahren, wo vielleicht Ärzte involviert sind. Dabei handelt es sich tatsächlich aber weniger um Ärzte, die wirklich Dopingmethoden anwenden, als vielmehr Ärzte, die möglicherweise ohne echte medizinische Indikation Dopingmittel verabreicht haben."

Bei den verwendeten Dopingmitteln handelte es sich überwiegend um Anabolika $\left(45,6 \%^{25}\right)$. Diuretika spielten lediglich in 2,9\% der Fälle eine Rolle. Stimulantien wurden in 25,2\% der Fälle relevant; hierunter fielen neben dem als Sportdroge bekannte DMAA auch etwa THC, Kokain, Ritalin oder Amphetamin. In 14,6\% der Verfahren waren Mittel von Bedeutung die selbst keine unmittelbar leistungssteigernde Wirkung entfalten sollten, sondern zur „Maskierung“ der unerlaubten Einnahme z.B. anaboler Steroide eingesetzt wurden.

Dopingmittel und -methoden in absoluten Zablen:

\begin{tabular}{|l|l|}
\hline Anabolika & 47 \\
\hline - davon Testosteron/-derivate & 23 \\
\hline Stimulantien & 26 \\
\hline - davon DMAA & 8 \\
\hline Maskierung & 15 \\
\hline Diuretika & 3 \\
\hline Methode & 4 \\
\hline
\end{tabular}

25 In einigen Fällen wurden verschiedene Mittel kombiniert (z.B. Anabolika und Stimulantien), sodass hier Mehrfachnennungen möglich sind. 
2. Materiell-rechtliche Fragen

a) Tathandlungen

aa) Legislativer Kontext

$\$ 4$ enthält ein differenziertes System strafbarer Handlungen, hinter dem unterschiedliche Unrechtskonzeptionen stehen. Während $\mathbb{\$} 4$ Abs. 1 Nr. 1-3 Verstöße gegen die in $\mathbb{2} 2$ enthaltenen Verbote sanktionieren, beziehen sich die in $\mathbb{S} 4$ Abs. 1 Nr. 4,5 und $\$ 4$ Abs. 2 enthaltenen Tathandlungen auf das von $\mathbb{3} 3$ verbotene Selbstdoping. Strafbar handelt danach, wer entgegen $₫ 3$ Abs. 1 S. 1 ein Dopingmittel oder eine Dopingmethode

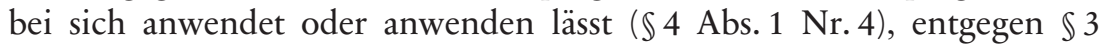
Abs. 2 an einem Wettbewerb des organisierten Sports teilnimmt ( $\$ 4$ Abs. 1 Nr. 5) sowie wer entgegen $₫ 3$ Abs. 4 ein Dopingmittel erwirbt oder besitzt ( $₫ 4$ Abs. 2).

Besonders umstritten war die von $₫ 4$ Abs. 1 Nr. 4 geschaffene Strafbarkeit des Selbstdopings, bei dem es sich nach Auffassung des Gesetzgebers um „einen tragenden Eckpfeiler der mit dem Anti-Doping-Gesetz verfolgten Dopingbekämpfungskonzeption“ handelt. ${ }^{26}$ Das Verbot der Teilnahme an einem sportlichen Wettbewerb unter Dopingeinfluss ist im Laufe des Gesetzgebungsverfahrens eingefügt worden, um Strafbarkeitslücken zu schließen, etwa in Fällen, in denen sich der Täter im Ausland selbst dopt, um im Inland an einem Wettbewerb teilzunehmen. ${ }^{27}$ Die Besitzstrafbarkeit erfasst auch geringe Mengen, wenn die Dopingmittel zum Zwecke des Selbstdopings erworben oder besessen werden. Dies sei, so die Gesetzesbegründung, „auch unter Berücksichtigung der Ultima-ratio-Funktion des Strafrechts gerechtfertigt, weil bereits mit dem Erwerb oder dem Besitz der Dopingmittel eine erhebliche Schutzgutgefährdung eintritt." Nur so sei es möglich, „effektiv gegen Dopingsünder vorzugehen und damit die Integrität des organisierten Sports zu schützen." 28

26 BT-Drs. 18/4898, S. 29.

27 Zustimmend MüKo-StGB/Freund, 3. Aufl. 2018, AntiDopG \$S 1-4 Rn. 36.

28 BT-Drs. 18/4898, S. 29. 
bb) Quantitative Auswertung

Die Auswertung der Akten ergab folgende Verteilung der Ermittlungsverfahren auf die selbstdopingbezogenen Tatbestandhandlungen.

Abb. 2: Verteilung der Verfahren nach Tathandlung gem. $\$ 4$

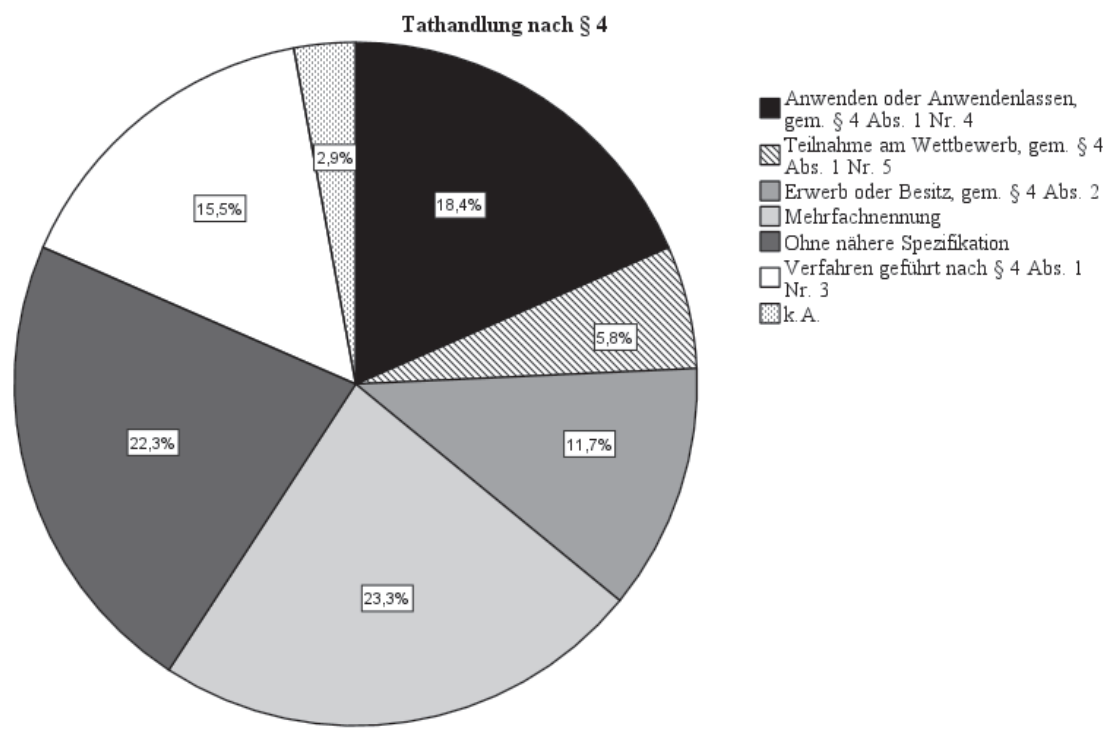

Die Tatvariante „Teilnahme an einem organisierten Wettbewerb“ ( $\$ 4$ Abs. 1 Nr. 5) ist deutlich unterrepräsentiert. In einem erheblichen Anteil der Verfahren fehlten Angaben zur Tathandlung oder waren nicht spezifiziert.

cc) Dopingmethoden

Auffällig in der Evaluierung war zum Ersten, dass die tatbestandlich erfassten Dopingmethoden in den Ermittlungen bislang keine Rolle spielten (siehe dazu bereits oben 1.c): Nur in drei Verfahren ging es ausschließlich um die Anwendung von Dopingmethoden (2,9\%); in einem Verfahren sowohl um Dopingmittel als auch um Dopingmethoden (1,0\%). Dennoch wird die Erfassung von Dopingmethoden allgemein als sinnvoll erachtet.

„Ich meine, dass es generell sinnvoll ist." (A1) 
dd) Präzision und Nachvollziehbarkeit der Tatbestandsformulierungen

Auffällige Unterschiede zeigten sich auch bei den Antworten auf die Frage, ob die Straftatbestände des Selbstdopings ausreichend präzise und nachvollziehbar formuliert seien. Während etwa StA 4 die Frage umstandslos mit " $J a$ " beantwortete, deutete ein Vertreter eines Verbandes Verständnisbzw. Vermittlungsschwierigkeiten bei bzw. gegenüber Athleten an:

„Natürlich ist das juristisches Hochreck. (...) Ich glaube, diese ganzen unterschiedlichen Tatbestände sind schon relativ kompliziert. Aber ich finde schon, dass man Athleten sehr gut erklären kann, was erlaubt ist und was nicht." $(D)$

In einem anderen Interview klangen die Bedenken noch stärker an:

„Grundsätzlich lässt sich damit arbeiten, wenngleich natürlich auch die Verweise innerhalb der Norm manchmal durchaus schwierig sind. Das merken wir auch dann, wenn wir Staatsanwaltschaften anschreiben, die eben keinen Schwerpunkt haben, dann fällt es manchmal selbst dem geübten Staatsanwalt schwer, tatsächlich die Verbindungsnorm und die entsprechenden Verweise richtig zu lesen. Also von daher könnte da sicherlich eine gewisse Klarheit nochmal dazu beitragen. " $(N)$

Besonders deutlich wird die Kritik bei einem befragten Richter:

„Es mag sein, dass sie ausreichend präzise formuliert sind. Praktisch sind sie sehr schlecht. Ich bin der Auffassung, dass Straftatbestände beim erstmaligen Durchlesen - jedenfalls für jemand, der schon seit vielen Jahren Strafrecht macht - sofort verständlich sein müssen. Denn sie müssen ja auch für den, an den sie sich richten, verständlich sein." (R1)

Die Auswertung der Akten bestätigt die Bedenken jedenfalls teilweise. Wie die Statistik (oben a) zeigt, machten die Staatsanwaltschaften in einer auffällig hohen Anzahl von Fällen keine oder keine spezifischen Angaben zur Tathandlung. Diese Verfahren wurden schlicht mit Verweis auf "\$4 AntiDopG“, „\$ 4 Abs. 1 AntiDopG“ oder gar „Verstoß gegen AntiDopG“ eingeleitet und teilweise auch fortgeführt, ohne dass erkennbar auf eine Tatvariante Bezug genommen oder wenigstens zwischen einem Verstoß gegen die Verbote des $\$ 2$ oder einem Fall des Selbstdopings gem. $\$ 3$ unterschieden wurde. Angesichts der Tatsache, dass die verschiedenen Tatvarianten des $\$ 4$ auf unterschiedlichen Unrechtskonzeptionen basieren und für sie andersartige Tatbestandsvoraussetzungen und Strafrahmen gelten, ist ein sol- 


\section{B. Ergebnisse der Evaluierung}

ches Vorgehen problematisch. Dementsprechend wenig zielgenau verliefen oftmals die Ermittlungen und wurden rasch eingestellt.

Auch die Mehrfachnennungen von Tathandlungen sind nicht unproblematisch: In nicht wenigen Akten fanden sich textbausteinartige Formulierungen wie „Selbstdoping; Anwenden oder Anwendenlassen von Dopingmitteln; Teilnahme am Wettbewerb“, teilweise kombiniert mit „Erwerb oder Besitz entgegen $\mathbb{3}$ Abs. 4 “. Solche Formulierungen kaschieren nur, dass der konkrete Bezugspunkt der Ermittlungen letztlich unklar ist.

Auch ein zweites Muster legt nahe, dass die Systematik der Tatbestände mit ihren verschiedenartigen Schutzzwecken und Anwendungsvoraussetzungen nicht immer im notwendigen Ausmaß nachvollzogen wird. So begründeten Staatsanwaltschaften den Anfangsverdacht einer Besitzstrafbarkeit nach $\$ 4$ Abs. 2 in einigen Fällen bereits mit der Bestellung eines Dopingmittels, obwohl weder die ausgeübte Sportart erkennbar war noch Erkenntnisse zu Wettkampfteilnahmen oder Einnahmen vorlagen. Die Verfahren wurden dann - in der Regel ohne nähere rechtliche Ausführungen - mit Verweis auf die Einmaligkeit des Vergehens und die geringe Schuld eingestellt. In einem Verfahren wurde dem Beschuldigten mitgeteilt:

„Da das mögliche Verschulden mir verhältnismäßig gering erscheint und ich davon ausgehe, dass es sich allenfalls um einen einmaligen Verstoß gegen ein Strafgesetz handelt, sehe ich ausnabmsweise von einer weiteren Verfolgung ab. Das gezeigte Verhalten rüge ich jedoch nachdrücklich. Im Wiederholungsfall können Sie mit einer erneuten Einstellung nicht rechnen." (Aktenauszug)

Eine solche Einstellungsbegründung kann dem Beschuldigten weder den Grund des Strafverfahrens noch die Konturen des strafbaren Verhaltens vermitteln, so dass spezialpräventive Ziele verfehlt werden. Vor allem aber begründet die Einstellungsbegründung auch Zweifel daran, dass sich der Staatsanwalt mit den gesetzlichen Anforderungen in der notwendigen Differenziertheit auseinandergesetzt hat.

Schließlich stellten die Staatsanwaltschaften in 17 Fällen auf $\$ 4$ Abs. 1 Nr. 3 ab, obwohl sich die Ermittlungen inhaltlich mit Selbstdoping befassten. 
ee) Mengenunabhängige Kriminalisierung des Erwerbes und Besitzes von Dopingmitteln

Der Erwerb oder Besitz auch einer geringen Menge von Dopingmitteln ist nach $\mathbb{} 4$ Abs. 2 strafbar, wenn dies dem Ziel des Selbstdopings ( $\$ 3$ Abs. 4) dient. Während des Gesetzgebungsverfahrens ist gelegentlich die Sorge geäußert worden, eine mengenunabhängige Kriminalisierung des Erwerbs und Besitzes von Dopingmitteln könne dazu führen, dass Fehler des Athleten oder seines medizinischen oder sportlichen Umfeldes bei der Beschaffung von Medikamenten zu Ermittlungsverfahren führen oder dass Konkurrenten vorsätzlich Dopingmittel bei einem Athleten platzieren, um diesem zu schaden. ${ }^{29}$ Diese Bedenken haben sich im Rahmen der Evaluierung nicht bestätigt. So sagte ein Athletenvertreter:

„Bisher ist mir keiner der geschilderten Fälle bekannt und deshalb will ich $z u$ diesem Zeitpunkt sagen, dass sich die Bedenken nicht bestätigt haben." (A2)

$\mathrm{Ob}$ die Kriminalisierung geringer Mengen auch praktisch notwendig ist, ist hingegen unklar. So sagte ein Staatsanwalt einer Schwerpunktstaatsanwaltschaft:

"Mir ist jetzt kein Fall aus meiner Praxis bekannt, in dem es darauf ankam." (StA 4)

Jedenfalls bei Spitzensportlern ist die Strafbarkeit von Besitz und Erwerb auch geringer Mengen von Dopingmitteln nach Auffassung eines anderen Ermittlers nicht relevant, da diese Dopingmittel typischerweise nicht selbst beschaffen, sondern sich diese von anderen (etwa Sportärzten) in deren Praxis oder an einem anderen Ort verabreichen lassen:

„Aber im Bereich des Testpools, die besitzen ja in der Regel selber nicht, sondern bekommen es zum Konsum praktisch übergeben oder werden praktisch unter ärztlicher Aufsicht dann versorgt." (StA 3)

Dennoch äußerten sich Staatsanwälte überwiegend zustimmend zur Ausgestaltung des Gesetzes:

„Der Wegfall der nicht geringen Menge beim Leistungssportler, finde ich schon, dass sich das praktisch bewährt hat. Zum einen war es gesetzgeberisch

29 Siehe etwa Stellungnahme Anti-Doping Gesetz durch die Athletenkommission im DOSB, 2015, S. 2. Siehe auch Peukert, npoR 2015, 95, 99. 
und auch vom Ziel gesetzten Zweck her war es ja so gewünscht, hat sich auch in meinem Gefübl und Empfinden so bewährt. Aber auch da ist es so, es vereinfacht halt die Strafverfolgung beim Leistungssportler, weil der Anfangsverdacht schneller bejaht werden kann, um halt dann strafprozessual alle Maßnahmen zu ergreifen. Wenn man mal Fälle in dem Bereich hat, reicht diese Formulierung, um einen Anfangsverdacht zu begründen, weil nämlich andererseits im Kraftsportbereich ja beim Leistungssportler nicht der Anfangsverdacht besteht, da es ja ein Dopingmittelbesitz ist, der über eine nicht geringe Menge hinausgeht." (StA 3)

„Aber gleichwobl sozusagen, um das nochmal rauszustellen, der Athlet darf eben überhaupt keine Dopingmittel besitzen, das halte ich durchaus für sinnvoll“ (StA 1)

Ein Staatsanwalt machte hingegen Bedenken geltend:

„Wir machen $\$ 153$ oder $\$ 153$ a StPO. Ich glaube, da wird zu viel kriminalisiert, was jetzt nicht unbedingt der Fall sein sollte. Vielleicht sollte man die nicht geringen Mengen nochmals anpassen" (StA 2)

\section{ff) Zusammenfassung}

Im Ergebnis lassen sich vernehmbare Zweifel an der Art der gesetzgeberischen Ausgestaltung feststellen, die sowohl quantitativ als auch durch zwei bei der Führung der Ermittlungen feststellbare problematische Muster bestätigt werden. Daher sollte über eine Vereinfachung der Tatbestandstrukturierung nachgedacht werden, insbesondere durch eine deutlichere Unterscheidung zwischen jenen Tatbestandsalternativen, die sich auf Verstöße gegen $\ 2$ beziehen, und solche zum Selbstdoping, für die überdies die täterbezogene Beschränkung durch Abs. 7 gilt. Nimmt man die Schwierigkeiten durch die Täterkreiseinschränkung nach $₫ 4$ Abs. 7 hinzu (dazu unten 2., b., bb), sollte eine Präzisierung der Straftatbestände erwogen werden (dazu unten C II 1 a). 
b) Weitere Strafbarkeitsvoraussetzungen

aa) Medizinische Indikation

\section{(1) Legislativer Kontext}

Doping meint die Anwendung von Dopingmitteln und -methoden zu einem aus medizinischer Sicht nicht angezeigten Eingriff in den Körper. ${ }^{30}$ Da viele Dopingmittel zugleich (zugelassene) Arzneimittel sind, muss das AntiDopG zwischen unzulässigen und verbotenen Anwendungen solcher Arzneimittel unterscheiden. Nach $₫ 3$ Abs. 1 liegt ein verbotenes und gem. \4 Abs. 1 Nr. 4 strafbares Selbstdoping daher nur dann vor, wenn ein Sportler Dopingmittel bzw. -methoden „ohne medizinische Indikation“ bei sich anwendet oder anwenden lässt. Wird ein Dopingmittel oder eine Dopingmethode zu therapeutischen Zwecken angewendet, ist das Verhalten sozialadäquat, selbst wenn damit als Nebenfolge eine Steigerung der sportlichen Leistungsfähigkeit einhergeht. ${ }^{31}$ Hier wirkt sich die Dualität der Schutzrichtungen des AntiDopG aus, das nicht nur die Integrität des Sports, sondern auch die Gesundheit der Sportlerinnen und Sportler schützt (vgl. \1). Dort aber, wo die Anwendung von Arzneimitteln medizinisch indiziert ist, ist für ein Verbot oder gar eine Bestrafung kein Platz. In der Literatur gilt die Unterscheidung zwischen einer medizinisch indizierten und einer nicht-indizierten Anwendung als „schwierig“. 32

30 Vgl. BT-Drs. 18/4898, S. 22; Weber BtMG/ders., 5. Aufl. 2017, AntiDopG $₫ 3$ Rn. 18.

31 Vgl. BT-Drs. 18/4898, S. 27. - Sportrechtlich ist für solche Fälle indes die Erteilung einer Medizinischen Ausnahmegenehmigung (Therapeutic Use Exemption, TEU) für den Einsatz verbotener Substanzen oder Methoden nach dem Welt AntiDoping Code (WADC) erforderlich; siehe dazu im Einzelnen den International Standard for Therapeutic Use Exemptions. Die Einnahme nicht verbotener Medikamente oder Substanzen ist bei der Dopingkontrolle auf dem Dopingkontrollformular anzugeben.

32 Lehner/Nolte/Putzke/Putzke, Anti-Doping-Gesetz, $\mathbb{3}$ Rn.10, mit der Begründung, dass sich nach Erkrankungen oder Unfällen ärztlich verschriebene Medikamente stets (und ggfs. bis in Wettbewerbe) leistungssteigernd auswirkten; dies verkennt aber, dass das Verbieten gerade nicht an der Leistungssteigerung ansetzt, sondern an der medizinischen Indikation. Was medizinisch indiziert ist, entscheidet der Stand der medizinischen Wissenschaft, zudem können Ausnahmegenehmigungen (TEU) eingeholt werden (siehe vorstehende Fußnote). 
(2) Erkenntnisse der Studie

Die Evaluierung hat vereinzelte Hinweise auf derartige Schwierigkeiten ergeben. Häufiger standen jedoch andere Tatbestandsmerkmale im Zentrum der Ermittlungen und die Verfahren wurden aus anderen Gründen (geringe Schuld, Auslandssachverhalt) eingestellt. Lediglich in einem Interview wurde die medizinische Indikation als problematischer Punkt in den Ermittlungen genannt:

„Bei der Häufigkeit ist es mit Sicherheit fehlende medizinische Indikation, weil natürlich im Breitensport auch häufig geltend gemacht wird, dass man die Präparate nicht zum Muskelaufbau oder zur Steigerung der Leistung im Breitensport bezieht, sondern aus medizinischen Gründen." (StA 7)

Allerdings schränkte derselbe Staatsanwalt seine Aussagen zum Selbstdoping-Verbot später ein:

„Da das so wenige Fälle sind, kann ich dazu keine valide Aussage treffen." (StA 7)

Nur in 25 Verfahrensakten fanden sich überhaupt - und zumeist kurze Hinweise darauf, dass die (fehlende) medizinische Indikation in den Blick genommen wurde. In 13 Fällen haben sich die Beschuldigten mit Hinweis auf eine medizinische Indikation verteidigt, sei es durch Vorlage eines entsprechenden Rezepts, sei es durch den allgemeinen Verweis auf eine Krankheit, ohne Vorlage einer ärztlichen Verordnung. Nur in einem Fall, in dem auch der Verein des Beschuldigten diverse medizinische Gutachten zu dem Spieler und seinen Erkrankungen vorlegte, wurden weitergehende Ermittlungen zur medizinischen Indikation angestellt; das Verfahren wurde letztlich aus anderen Gründen eingestellt.

In der Mehrheit der Verfahren prüfte die Staatsanwaltschaft das Tatbestandsmerkmal nicht näher. So lag in vier Fällen die fehlende Indikation auf der Hand, weil die Staatsanwaltschaft wegen der Einnahme von Kokain ermittelte, das in Deutschland kein zugelassenes Arzneimittel und - so die Ermittler - auch nicht Bestandteil zugelassener Arzneimittel ist. In zwei Verfahren räumten die Beschuldigten (von vornherein bzw. auf Nachfrage der Ermittler) ein, dass keine medizinische Indikation vorlag. Deutlich häufiger finden sich in den Akten jedoch Einschätzungen der NADA zur fehlenden Indikation, etwa der Hinweis, dass in Deutschland „derzeit keine Arzneimittel mit Dehyrochlormethyltestosteron als Wirkstoff im Humanbereich" zugelassen seien oder dass eine versehentliche Einnahme durch Medikamente „fast gänzlich ausgeschlossen“ sei. Ebenso 
verbreitet sind Hinweise der NADA auf das Fehlen oder Vorhandensein einer „Therapeutic Use Exemption“ bzw. Ausnahmegenehmigung. ${ }^{33}$

bb) Vorteilsverschaffungsabsicht in einem Wettbewerb des organisierten Sports

Der Täter muss an einem „Wettbewerb des organisierten Sports“ teilnehmen ( $\$ 3$ Abs. 2) oder die Absicht haben, sich durch das Selbstdoping einen Vorteil in einem solchen Wettbewerb zu verschaffen ( $\$ 3$ Abs. 1). Nach der Legaldefinition in $\$ 3$ Abs. 3 gilt als Wettbewerb des organisierten Sports jede Sportveranstaltung, die

1. von einer nationalen oder internationalen Sportorganisation oder in deren Auftrag oder mit deren Anerkennung organisiert wird und

2. bei der Regeln einzuhalten sind, die von einer nationalen oder internationalen Sportorganisation mit verpflichtender Wirkung für ihre Mitgliedsorganisationen verabschiedet wurden.

(1) Auslegung des Merkmals „Wettbewerb des organisierten Sports“

Durch das Tatbestandsmerkmal sollen private Turniere vom Anwendungsbereich des Verbots ausgenommen werden, da Selbstdoping im Freizeitbereich die Integrität des organisierten Sports nicht in einem Maße beeinträchtigt, das eine Strafbarkeit rechtfertigt. ${ }^{34}$ Nach der Gesetzesbegründung zählen zu den erfassten Wettbewerben „in erster Linie Sportwettbewerbe des Spitzen- und Leistungssports, wie Olympische und Paralympische Spiele oder Jugendspiele, Weltspiele (World-Games), nationale oder internationale Meisterschaften, Spiele oder Wettkampfbetriebe einer nationalen oder internationalen Liga, nationale oder internationale Pokalwettbewerbe oder internationale Freundschaftsspiele. Erfasst sind aber auch größere Laufveranstaltungen (z. B. Marathon) und regionale Ligen, Sportfeste und Sportveranstaltungen privater Veranstalter, wenn und soweit die-

33 Bei bestimmten Krankheitsbildern können die NADA bzw. die internationalen Sportdachverbände eine Ausnahmegenehmigung (Therapeutic Use Exemption, TEU) für den Einsatz verbotener Substanzen oder Methoden nach dem Welt AntiDoping Code (WADC) erteilen.

34 BT-Drs. 18/4898, 27; Erbs/Kohlhaas/Wußler, 232. EL August 2020, AntiDopG $₫ 3$ Rn. 9. 


\section{B. Ergebnisse der Evaluierung}

se von den jeweils zuständigen (nationalen oder internationalen) Sportorganisationen im Vorfeld anerkannt worden sind." ${ }^{35}$ Organisiert werden muss der Wettkampf von einer nationalen oder internationalen Sportorganisation, also einer juristischen Person oder einem Personenverband, deren Hauptaufgabe die Förderung sportlicher Betätigungen ist. ${ }^{36}$ Voraussetzung ist ein „gewisser Organisationsgrad“; zudem sind rein lokale Vereine nicht erfasst.

Nicht unter $₫ 3$ fallen daher etwa „reine Firmenläufe, Freizeitkickerturniere, rein privates Sporttreiben (z. B. Jogging im Park) oder Wettbewerbe, die ausschließlich im Rahmen des Schulsports (z. B. Spiele verschiedener Schulen gegeneinander) ausgetragen werden“. Im Schrifttum wird diskutiert, ob das Wettkampfbodybuilding dem Begriff des organisierten Wettbewerbs unterfällt. ${ }^{37}$

In 21 Fällen enthielten die Akten Angaben zum organisierten Wettbewerb; in den übrigen 82 Verfahren wurde das Tatbestandsmerkmal nicht thematisiert. In neun Verfahren beruhte die Annahme eines organisierten Wettbewerbs auf einer Mitteilung der NADA, die Dopingkontrollen im Rahmen von Wettbewerben durchführte.

In einem Verfahren trat die Staatsanwaltschaft an die NADA mit der Frage heran, ob es sich bei einer Pre-Season-Begegnung bereits um einen Wettbewerb des organisierten Sports i.S.d. $\$ 3$ Abs. 3 handele. Die NADA bejahte das Vorliegen eines Wettbewerbs mit der Begründung, dass es sich zwar um Spiele handele, „die dem Grunde nach zur Vorbereitung vor der eigentlichen Saison gehören“, diese Spiele allerdings "gerade nicht den Charakter von Freundschaftsspielen, bei denen ausschließlich das ,private Sporttreiben der Beteiligten' oder der Spaß an einer reinen Freizeitgestaltung im Vordergrund steht [haben]. Vielmehr geht es in den Begegnungen der Pre-Season um die gezielte und unmittelbare Saisonvorbereitung unter ,nahezu wettkampfgleichen Bedingungen“". Die Staatsanwaltschaft übernahm die Einschätzung der NADA.

In vier Verfahren wurde das Vorliegen eines Wettbewerbs mit Hinweis darauf bejaht, dass es sich um Bundeligaspieler handelte.

35 BT Drs. 18/4898, S. 28.

36 Erbs/Kohlhaas/Wußler, 232. EL Oktober 2020, AntiDopG $\$ 3$ Rn. 10.

37 MüKo-StGB/Freund, 3. Aufl. 2018, AntiDopG $\$ \$ 1$ 1-4 Rn. 39. Gegen die Anwendbarkeit von $₫ 3$ auf das Bodybuilding wird eingewandt, dass es sich hierbei nicht um eine Sportart, sondern um die Zurschaustellung von Körpern handele; Brill, SpuRt 2015, 153, 154; dazu Samson-Baudisch, Der Missbrauch von anabol-androgenen Steroiden im freizeitorientierten Bodybuilding, 2014, S. 18. Anders aber BGH NStZ 2010, 170. 
In sechs Fällen führte die Staatsanwaltschaft selbst Internetrecherchen durch und prüfte etwa über die Facebook-Seite der Beschuldigten, ob diese an Wettbewerben teilnahmen. Auf dieser Grundlage wurde in einem Fall ausdrücklich - allerdings ohne rechtliche Prüfung - die Teilnahme an Wettkämpfen des Bodybuildings für tatbestandsmäßig erklärt. In allen anderen gegen Bodybuilder geführten Verfahren wurde die Frage, ob Wettbewerbe in diesem Bereich in den Anwendungsbereich von $\$ 3$ fallen, nicht erörtert.

Die Auslegung des Wettbewerbsbegriffs wurde auch in den Interviews als ein Problem bei der Anwendung von $₫ 3$ genannt.

„Bei Veranstaltungen des organisierten Sportes da gibt es durchaus Streitmöglichkeiten in so, ich sag mal so Nischenbereichen. Ich denke da an eine größere Fight-Night zum Beispiel, die irgendwo organisiert wird. Oder man denkt an einen Stadtmarathon, jetzt in einer kleineren Stadt, wo man durchaus genau hingucken muss." (StA 1)

„Diese Definition ist natürlich schwierig. Was ist eine Sportorganisation? Wenn ich jetzt einen Wettbewerb von Bodybuildern habe. Beispielsweise Mr. und Mrs. Universe. Sind wir da im Anwendungsbereich? Ist natürlich eher schwierig. Es gibt natürlich in diesem Sinne keine Sportorganisation meiner Kenntnis nach im Bodybuildingbereich." (StA 5)

Gerade im Bereich des Bodybuildings zeigte sich eine unterschiedliche Vorgehensweise der Staatsanwaltschaften. Während der Wettbewerbscharakter meist offen blieb (und das Verfahren aus anderen Gründen eingestellt wurde, hierzu unten 3., c.), führten einige Staatsanwaltschaften Verfahren im Bereich des Bodybuildings grundsätzlich nicht wegen Selbstdopings, sondern allein wegen $\$ 2$.

„Wir prüfen $\$ 3$ hier gar nicht. Weil der letztendlich ja voraussetzt, dass man sich in einem Wettbewerb des organisierten Sports einen Vorteil verschaffen muss (...). Das war schon geklärt, bevor ich hier mit meiner Tätigkeit angefangen habe. Das hat die NADA hier schon gesagt. Das spielt für uns keine Rolle. (...) Wir lassen diese Verfahren dann unter dem Rabmen des $\$ 2$ laufen. Die Frage, ob jemand an Wettbewerben teilgenommen hat oder nicht, kann man natürlich strafschärfend werten im Verfahren. Da lässt der $\$ 2$ ja genug Raum." (StA 5) 
(2) Absicht einer Vorteilsverschaffung

Der Beschuldigte wird im Rahmen einer allgemeinen Wettkampfkontrolle anlässlich einer Bundesligabegegnung positiv auf Kokain getestet. Der Sportler ist Mitglied eines allgemeinen Testpools der NADA (ATP). Der Beschuldigte ist bereits in der ersten Vernehmung durch die Polizei geständig und erklärt, er habe aus privaten Gründen mit dem Gebrauch von Kokain begonnen, es aber niemals zur Leistungssteigerung im Wettkampf eingesetzt. Er sei seit einiger Zeit wegen seiner Kokainabhängigkeit in Therapie. Die Staatsanwaltschaft sieht die Schuld als gering an und stellt das Verfabren nach $\$ 153$ StPO ein.

Die Einnahme eines Dopingmittels muss mit der Absicht erfolgen, sich einen Vorteil in einem Wettbewerb des organisierten Sports zu verschaffen ( $(3$ Abs. 1 und 2). Während die Vorteilsverschaffungsabsicht in den meisten Fällen bereits durch die Art des Mittels indiziert wird, können insbesondere bei der Einnahme von Stimulanzien auch andere - teilweise nachvollziehbare - Motive in Betracht kommen. In mehreren Verfahren bestritten die Beschuldigten, die Mittel zur Erlangung eines sportlichen Vorteils eingenommen zu haben.

In dem eingangs skizzierten Verfahren überprüfte die Staatsanwaltschaft die Angaben des Beschuldigten nicht weiter, sondern stellte das Verfahren nach $\int 153$ StPO wegen geringer Schuld ein. Auch in den Interviews berichteten Staatsanwälte, dass eine Vorteilsverschaffungsabsicht von Beschuldigten bestritten wird. Entsprechende Einlassungen würden allerdings überwiegend als Schutzbehauptungen bewertet.

„Was ein Problem sein kann, ist die Frage, ob diese Mittel zu Dopingzwecken im Sport benutzt wurden. Es kommt nicht selten oder immer einmal wieder die Einlassung, das sei nicht zu Dopingzwecken im Sport, sondern zur Steigerung der Potenz, zur Linderung von Schmerzen. Darüber kann man diskutieren. Aus unserer Sicht sind es in der Regel Schutzbehauptungen, Versuche, wieder aus der Strafbarkeit herauszukommen. Aber das sind Punkte, über die gestritten wird. " (StA 4)

Allerdings werden die Einnahme eines Mittels oder die Anwendung einer Methode erst dann zu „Doping“, wenn sie tatsächlich leistungssteigernd eingesetzt werden. Das Merkmal der Vorteilsverschaffungsabsicht kann hier also ein Korrektiv bilden, um den Tatbestand auf tatsächlich dopingrelevante Verstöße zu beschränken. In einem Verfahren wurde dem Beschuldigten vorgeworfen, sich eine Infusion mit mindestens 100 Millilitern Flüssigkeit zum „Flüssigkeitsausgleich nach dem Gewichtmachen" für 
einen Ringerwettkampf gelegt zu haben. Gegen den Beschuldigten erging ein Strafbefehl, in der Hauptverhandlung wurde er jedoch freigesprochen, da ihm eine Vorteilsverschaffungsabsicht nicht nachzuweisen sei. Das Gericht kam zu dem Ergebnis, dass es „dem Angeklagten überhaupt keinen Vorteil für den Wettkampf [...] gebracht hätte, wenn er sich [...] zwei Tage vor dem Wettkampf und mindestens einen Tag vor dem Wiegen Flüssigkeit mittels Infusion hätte verabreichen lassen. Somit bleibt nach der durchgeführten Beweisaufnahme fraglich, wo für den Angeklagten der Vorteil [...] einer über $100 \mathrm{ml}$ Infusion sein sollte. Dieser Vorteil ist eine Tatbestandsvoraussetzung.“

\section{(3) Zwischenergebnis}

Die Untersuchung zeigt, dass das Merkmal des organisierten Wettbewerbs in der praktischen Anwendung bislang keine Konturen erhalten hat. Das Vorliegen eines organisierten Wettbewerbs ist fast durchweg nicht Gegenstand einer materiell-rechtlichen Prüfung. Dabei wäre es gerade im Bereich des Bodybuildings erforderlich, den Bezug zu einem sportlichen Wettbewerb zu untersuchen.

Die Vorteilsverschaffungsabsicht kann insbesondere bei der Einnahme illegaler Drogen zweifelhaft sein. Substanzen wie Kokain haben einen psychisch wie physisch leistungssteigernden Effekt und können daher unmittelbar vor einem Wettkampf zur Leistungssteigerung eingesetzt werden. Gleichzeitig ist Kokain eine verbreitete „Partydroge“, deren privater Konsum nicht außerhalb des Plausiblen liegt. Ein eindeutiger Nachweis der Vorteilsverschaffungsabsicht ist bei einem Bestreiten des Beschuldigten kaum möglich.

c) Strafbarkeitseinschränkungen in $₫ 4$ Abs. 7

$\$ 4$ Abs. 7 schränkt den Kreis möglicher Täter aus Gründen der Verhältnismäßigkeit ein. Auch diese Einschränkung wird auf den Gedanken gestützt, dass Manipulationen durch Doping nur dann „zu einem Vertrauensverlust in das Sportsystem und relevanten Schäden" führen, wenn es sich bei den Tätern um „herausgehobene Sportlerinnen und Sportler“ handele, die 
„ihren Sport leistungs- und wettkampforientiert auf hohem Niveau betreiben bzw. erhebliche Einnahmen aus der sportlichen Tätigkeit ziehen“..38

Es können sich damit nur Personen strafbar machen, die entweder Spitzensportler des organisierten Sports sind ( $\$ 4$ Abs. 7 Nr. 1) oder die aus der sportlichen Betätigung unmittelbar oder mittelbar Einnahmen von erheblichem Umfang erzielen ( $\$ 4$ Abs. 7 Nr. 2$).{ }^{39}$

aa) Testpool-Athleten

Als Spitzensportler gilt, wer als Mitglied eines Testpools im Rahmen des Dopingkontrollsystems Trainingskontrollen unterliegt. 11,7\% der Beschuldigten waren Mitglied eines Testpools der NADA, 74,8\% waren keine Testpool-Athleten und in 13,6\% der Verfahren erfolgte hierzu keine Angabe. Ob ein Athlet einem Testpool angehörte, wurde in allen einschlägigen Fällen bereits in der Anzeige der NADA mitgeteilt: „[Der Beschuldigte] unterfällt dem Anwendungsbereich der Sanktionsvorschriften des $\$ 4$ Abs. 7 Ziffer 1 AntiDopG. Er ist Mitglied des Testpools der NADA im Rahmen des Dopingkontrollsystems und unterliegt Trainingskontrollen."

Einer der befragten Staatsanwälte kritisierte, dass es den Ermittlungsbehörden nicht möglich sei, selbständig zu überprüfen, ob der Beschuldigte Mitglied eines Testpools sei.

„Wenn [an den Testpool] die Tätereigenschaft anknüpft, dann müsste es für Strafverfolgungsbehörden auch möglich sein, in der Tat genau überprüfen zu können: Wer ist denn in diesem Testpool? Da müsste es eine Zugriffsmöglichkeit für Strafverfolgungsbehörden geben (...). Wenn der Strafverfolger aber nicht selbständig überprüfen kann, ob eine bestimmte Person Mitglied eines Testpools ist, dann kann er ja letztendlich diese Strafbarkeit nicht verfolgen." (StA 7)

38 BT-Drs. 18/4898, S. 31

39 Teilweise wird vertreten, dass der Begriff des Spitzensportlers sowohl TestpoolAthleten als auch Sportler und Sportlerinnen mit Einnahmen von erheblichem Umfang erfasst, siehe etwa Erbs/Kohlhaas/Wußler, 228. EL Januar 2020, AntiDopG $₫ 4$ Rn. 5. Letztlich kommt es auf die begriffliche Zuordnung nicht an. 
bb) Einnahmen von erheblichem Umfang

(1) Teleologische Angemessenheit der Strafbarkeitseinschränkung

Nicht nur Sportler, die Trainings- und Wettkampfkontrollen unterworfen sind, können einem besonderen Anreiz ausgesetzt sein, verbotene Dopingmittel bzw. Dopingmethoden zu nutzen. Ein solcher Anreiz besteht vielmehr auch dann, wenn mit dem Sport Einnahmen von erheblichem Umfang generiert werden. Nach Auffassung des Gesetzgebers sollen die Strafvorschriften des Selbstdopings ( $\$ 4$ Abs. $1 \mathrm{Nr} .4,5$ ) sowie des Erwerbes und Besitzes von Dopingmitteln zur Vorteilsverschaffung im Wettbewerb ( $\$ 4$ Abs. 2 i.V.m. $\$ 3$ Abs. 4) daher auch Sportler erfassen, die aus ihrer sportlichen Betätigung unmittelbar oder mittelbar Einnahmen von erheblichem Umfang erzielen. Der Gesetzgeber begründet die Strafbarkeitseinschränkung zudem damit, dass auch diese Sportler Vorbilder seien und das Vertrauen in Anspruch nehmen, ,ihre sportlichen Erfolge mit lauteren Mitteln erlangt zu haben. " 40 Als Beispiele nennt die Gesetzesbegründung den organisierten Motorsport, das Profiboxen sowie die 3. Fußball-Liga der Herren. Auch dort stünden die Sportler typischerweise mit ihren sportlichen Leistungen in der Öffentlichkeit und könnten die Integrität des organisierten Sports untergraben.

Diese Strafbarkeitseinschränkung wird in der Literatur als willkürlich und mit dem Gesetzeszweck schwer vereinbar bezeichnet, ${ }^{41}$ von der Praxis aber als Ergebnis einer Ermessensausübung des Gesetzgebers akzeptiert:

„Das ist manchmal schwierig zu bestimmen, aber ich denke, es ist so gewollt, dass es auf diesen Kreis zugeschnitten ist. Das ist ja eine politische Frage, wen man davon erfasst haben möchte. Das ist möglicherweise schwierig zu bestimmen, das ist eine praktische Frage, die sich auch woanders ergibt." (StA 4)

(2) Anwendungsschwierigkeiten

Stimmen in der Literatur kritisieren den Begriff der „Einnahmen von erheblichem Umfang“ ferner wegen seiner unzureichenden Bestimmtheit. ${ }^{42}$

40 Dazu und zum Folgenden BT-Drs. 18/4898, S. $31 \mathrm{f}$.

41 Zum Ganzen MüKo-StGB/Freund, 3. Aufl. 2018, AntiDopG \$\$ 1-4 Rn 112 f.

42 Eising, Die Strafbarkeit des Eigendopings, 2018, S.210, der eine Klarstellung durch den Gesetzgeber fordert. 
Tatsächlich bereitet die Gesetzesformulierung in der Anwendung nicht unerhebliche Probleme. Auf die Frage, was die größten rechtlichen Schwierigkeiten bei der Beweisführung in Selbstdoping-Fällen seien, antwortete ein Staatsanwalt:

„Also im Bereich Spitzensport ist es sicherlich der $\$ 4$ Abs. 7, wenn wir eben keinen Athleten haben, der entsprechend in den Testpool fällt. (...) Einkommen von einer gewissen Erheblichkeit, diesen Nachweis zu führen, das ist oftmals schwierig. Dass man sagen muss, naja man kommt vielleicht jetzt doch an die Grenze, dass es eben nicht zu bejahen ist, das ist sicherlich schwierig (...). “ (StA 1)

In den ausgewerteten Verfahrensakten fanden sich nur wenige Beschuldigte, die eine Sportart auf einem Niveau ausüben, ab dem sich typischerweise eine größere Öffentlichkeit für sie interessiert (je ein Profi- und Kickboxer, die früher internationale Titel errungen hatten, sowie ein BundesligaRinger und ein Bundesliga-Gewichtheber). Häufiger richteten sich die Ermittlungen gegen weniger erfolgreiche Sportler (Kickboxer, Triathlet, Bodybuilder). Im Fall eines Arm-Wrestlers lässt sich ein Zeuge mit den Worten ein:

„Ich kann es [dass Beschuldigter erhebliche Einnabmen erzielt] mir auch nicht vorstellen, da bei uns Geld keine große Rolle spielt." (Aktenauszug)

Quantitativ sind Verfahren, in denen gegen Sportler mit Einnahmen von erheblichem Umfang ermittelt wurde, eher unterrepräsentiert. Zwar bestand in knapp der Hälfte der einschlägigen Verfahrensakten $(48,5 \%)$ der Verdacht, dass der Sportler bzw. die Sportlerin derartige Einnahmen erzielen. Tatsächlich feststellbar waren solche Einnahmen indes nur in 12,6\% der Fälle; ${ }^{43}$ und das, obwohl Staatsanwaltschaften ihrer Arbeit ein niedrigschwelliges Verständnis von „erheblichen Einnahmen“ zugrunde legen.

43 Dennoch endeten diese Verfahren in ihrer großen Mehrzahl mit Einstellungen, da in anderer Hinsicht kein hinreichender Tatverdacht bestand, die Schuld gering war oder sich die Beschuldigten in das Ausland abgesetzt hatten und ein Rechtshilfeersuchen mangels Strafbarkeit im Aufenthaltsstaat keinen Erfolg versprach. 


\section{(3) Bestimmung und Bestimmbarkeit der Formulierung}

Die Gesetzesbegründung nennt als Voraussetzung „das wiederholte Erlangen wirtschaftlicher Vorteile“; dabei müsse es sich um „maßgebliche Leistungen" handeln, die deutlich über eine bloße Kostenerstattung hinausgehen." 44 In der Literatur wird auf eine einzelfallbezogene Betrachtung ${ }^{45}$ bzw. sportartspezifische Anwendung abgestellt, der zufolge ein Sportler, der eine kostenintensive Sportart ausübe, auch größere Einnahmen erzielen können solle. ${ }^{46}$ Eine solche einzelfallbezogene Auslegung ließ sich in der Evaluation nicht feststellen. Vielmehr geht aus mehreren Verfahrensakten hervor, dass die Ermittlungsbehörden danach gefragt haben, ob Einnahmen in einer sozialversicherungspflichtigen Höhe erzielt wurden („450 Euro"). Auch in den Interviews bestätigten zwei Staatsanwälte, dass ihnen diese Summe als Anhaltspunkt diene. Ein anderer Staatsanwalt zieht Einnahmen und vermutete Ausgaben zusammen und operiert dann mit einem Betrag von ,im Bereich 900-1000 € (...) im Monat“ (StA 3).

Es klingt jedoch auch Unsicherheit über die Angemessenheit von vergleichsweise niedrigen Einnahmengrenzen an:

„Ein Problem hatte ich letztens mit der Frage, was Einnahmen von erheblichem Umfang sind. Der [Beschuldigte] war vom Football, hat $1.000 € \mathrm{im}$ Monat verdient, hat aber natürlich auch Kost und Logis zur Verfügung gestellt bekommen. Da habe ich mich schon gefragt: Hat jetzt ein FootballSpieler, der in der 1. Bundesliga spielt und nur $1.000 €$ im Monat bekommt, Einnahmen von erheblichem Umfang, ja oder nein? Soweit ich gesehen habe, gab es auch keine Rechtsprechung dazu. Deswegen wäre es schon interessant, wenn der Gesetzgeber das näher konkretisieren könnte." (StA 1)

Auch andere Befragte wünschten sich, dass der Gesetzgeber oder die höchstrichterliche Rechtsprechung begriffliche Klarheit schafft.

„Was da natürlich wünschenswert wäre, auch das wäre vielleicht eine Idee fürs AntiDopG, sich mal zu überlegen, ob man da einem nicht irgendwo eine Marge an die Hand gibt. Oder vielleicht kann man es auch der Ausgestaltung durch die Rechtsprechung überlassen (...).“ (StA 3)

44 BT-Drs. 18/4898, S. $31 \mathrm{f}$.

45 Erbs/Kohlhaas/Wußler, 228. EL Januar 2020, AntiDopG $\$ 4$ Rn. 8.

46 Lehner/Nolte/Putzke/Putzke, Anti-Doping-Gesetz, $\mathbb{4}$ Rn. 33, der im Übrigen aus durchschnittlichen Gehaltszahlungen von (Herren-)Fußballspielern der 3. Liga und der Regionalligen die Grenze bei Jahreseinnahmen von netto 18.000 Euro bzw. dreimaligen Zahlungen von 1.500 Euro zieht. 
"Ja, ich denke, insgesamt ist es [das AntiDopG] schon geglückt. Die Fälle im Spitzensport halten sich ja bisher noch in Grenzen, wenn ich das richtig verstanden habe, aber ich glaube schon, dass es insgesamt geglückt ist. (...) Was für uns wichtig ist (...), ist diese Spezifizierung des Einkommens. “(A 2)

Die Instanzgerichte scheinen bislang noch keine Erfahrung mit diesem Tatbestandsmerkmal gemacht zu haben:

„Nein, das haben wir noch nicht angewendet. Weiß ich nicht. Der Begriff ist unglücklich gewählt, weil er hinsichtlich dieser Einnahmen möglicherweise eine neue Kategorie im Strafrecht einführt. Ich wüsste jedenfalls keinen Straftatbestand sofort, wo dieses Kriterium auch auftaucht." ( $R$ 1)

Ein Vertreter des Verbandssports $(D)$ hält die Formulierung weiterhin für „zu unbestimmt“. Es überrascht daher nicht, dass die niedrige Schwelle, ab der Staatsanwaltschaften von erheblichen Einnahmen ausgehen, im Bewusstsein von Sportlerinnen und Sportlern noch nicht verankert zu sein scheint.

„Ehrlich gesagt, habe ich das so verstanden, dass das eigentlich sowieso dadurch nur Bundeskader-Athleten betreffen kann inkl. der Siegersportarten, also Nationalmannschaften, Bundeskader plus Ligabetrieb: Fußball, Basketball, Handball. Ich finde es schwierig, das zu beziffern. (...) Ich finde eine Zabl schwierig. Ich finde eher, man sollte sagen: auf professionellem, internationalem Level im Bundeskader oder Bundesligen. Die Bundesliga ist ja, glaube ich, immer die Grenze, ob jemand professioneller Spieler ist oder nicht und damit von seinem Gehalt leben kann. Da würde ich es vielleicht beziffern. Dass es ein Lebensunterhalt ist." (A 1)

Ein anderer Athletenvertreter plädierte für einen deutlich höheren Schwellenwert:

„Ein Kriterium bei der Sporthilfeförderung ist z.B. $45.000 €$ pro Jahr. Das wird (...) als Kriterium genommen dafür, ob jemand Förderung erbalten soll oder nicht. Ich glaube, das wäre als erste Orientierung ganz hilfreich. "(A 2)

\section{(4) Ermittlungen}

Durchsuchungs- und Beschlagnahmebeschlüsse werden zumeist auf die nicht näher begründete Annahme gestützt, dass der Beschuldigte erhebliche Einnahmen erziele. Beispielsweise stellte die Staatsanwaltschaft in einem gegen eine Bodybuilderin geführtem Verfahren lediglich fest: 
„Wobei sie in der Kategorie Frauen Physique den vierten Platz belegte. Sie erzielt daher durch ibre sportliche Betätigung nicht unerhebliche Einnahmen." (Aktenauszug)

Nähere Ausführungen zur Höhe der Einnahmen enthält die Ermittlungsakte indes nicht. Gelegentlich wurden im Vorfeld Internet-Recherchen zum Beschuldigten, einer etwaigen Anstellung und möglichen Sponsoren durchgeführt. Eine große Rolle scheint zudem die kriminalistische Erfahrung zu spielen. So wird in Verfahren gegen Kraftsportler oft gar nicht erst wegen eines Verstoßes gegen $\$ 3$ ermittelt, da viele Staatsanwälte davon ausgehen, dass diese weder Spitzensportler seien noch erhebliche Einnahmen erzielten.

"[D]as sind so die Erfahrungen, die man im Lauf der Jahre sich aneignet, d.h. Kraft-, Kraftdreikämpfer und Bankdrücker, Steinlupfer und wie sie alle heißen oder Armdrücker: Wenn man da durchsucht [..., zeigt sich], dass die alle zwar in geordneten und stabilen, aber nicht in Verhältnissen leben, die Angriffspunkte dafür bieten, dass hier erhebliche Einnahmen erzielt werden (...).“(StA 3)

Auch bei der Aktenauswertung zeigte sich, dass die Ermittlungen gegen Kraftsportler schnell auf Verstöße gegen $₫ 2$ fokussiert werden.

„Beim Kraftdreikämpfer, bei dem eine Anzeige über die NADA reinkommt, den wird man in der Regel auch überführen können, aber halt nicht über den $\$ 3$, sondern über den $\$ 2$, weil der eine nicht geringe Menge in der Regel besitzt." (StA 3)

Im weiteren Fortgang der Ermittlungen werden zumeist einfache Ermittlungsmaßnahmen durchgeführt. Dazu zählt die Auswertung von beschlagnahmten Kontoauszügen oder Arbeits-, Anstellungs- oder Sponsorenverträge sowie die Befragung des Beschuldigten und von Zeugen. Aufwändigere Finanzermittlungen durch die Einbeziehung von Banken kommen nur selten vor. Sie wurden dann betrieben, wenn der Fall dazu besonderen Anlass bot: In einem Verfahren schien dies der (zusätzliche) Bezug zu Verstößen gegen das BtMG gewesen zu sein, in anderen Fällen offenbar das kriminalistische Gespür der Ermittler, dass der Beschuldigte erhebliche Einnahmen erzielt hatte und auch die übrigen Tatbestandsmerkmale erfüllt sind.

In einem Fallbeispiel, in dem die Staatsanwaltschaft breit ermittelt und auch frühzeitig Finanzermittlungen durchgeführt hat, hatte zunächst die NADA über eine positive Dopingprobe am Rande der Deutschen Meister- 


\section{B. Ergebnisse der Evaluierung}

schaften im Kickboxen berichtet. Das Ermittlungsverfahren ist zunächst wegen $\$ \int 4$ Abs. 1 Nr. 3, 2 Abs. 3 geführt worden, da der Beschuldigte kein Testpool-Athlet war und auch erhebliche Einnahmen jedenfalls nicht offensichtlich vorlagen. Auf dieser Grundlage werden eine Durchsuchung und - durchaus ungewöhnlich - auch eine Blutentnahme $(\mathbb{S} 81 \mathrm{a}$ StPO) angeordnet. Letztere war für den Nachweis einer Besitzstrafbarkeit zwar nicht notwendig, aber rechtlich zulässig und hätte vor allem den Nachweis des Selbstdopings erleichtert, für den Fall, dass der Beschuldigte erhebliche Einnahmen erzielt und damit in den Anwendungsbereich des $\mathbb{} 4$ Abs. 1 Nr. 4, 5 gelangt. Um dies zu prüfen, hat die Staatsanwaltschaft durch die Anfrage bei Banken ermittelt, welche Einkünfte der Beschuldigte erzielt hatte. Dies hat sich letztlich nicht feststellen lassen, so dass ein Strafbefehl wegen $\int S 4$ Abs. 1 Nr. 3, 2 Abs. 3 beantragt worden ist. Ein solcher Ermittlungsaufwand ist indes nur in einer geringen Anzahl von Verfahren betrieben worden.

\section{(5) Zusammenfassung}

Zusammenfassend lässt sich festhalten, dass das Tatbestandsmerkmal trotz bzw. gerade wegen einer recht niedrig angesiedelten Schwelle - praktische Probleme bereitet. Den Sportlern scheint vielfach nicht klar zu sein, dass bereits Einnahmen in einer Höhe, die eine Sozialversicherungspflicht auslösen würden (450€), einen entsprechenden Anfangsverdacht begründen können. Auch Strafverfolger wiesen ausdrücklich auf praktische Schwierigkeiten hin oder ließen diese zumindest anklingen; mehrere Befragte wünschten sich ausdrücklich eine gesetzgeberische oder höchstrichterliche Klarstellung. Deutlich zeigt sich, dass der von den Staatsanwaltschaften weithin verwendete niedrige Schwellenwert dazu führt, dass auch gegen Sportler wegen Verstößen gegen das Selbstdopingverbot ermittelt wird, die nur geringe Einnahmen erzielen und nicht im Licht der Öffentlichkeit stehen. Dem geringeren Unrecht solcher Taten und dem dadurch entstehenden Missverhältnis zu weiteren Ermittlungen tragen die Staatsanwaltschaften (wie in anderen Fällen kleinerer Delinquenz) mit dem Mittel der Verfahrenseinstellung Rechnung.

Gerade Ermittlungen gegen Sportler mit Einnahmen an der Grenze zur Erheblichkeit können für die Staatsanwaltschaften aufwändig sein: Geringere Summen sind nicht nur per se weniger auffällig als größere Beträge. Leistungen kleineren Umfangs sind auch nicht immer als Zahlungseingänge auf einem Konto sichtbar, sondern können bspw. als Übernahme der 
Kosten für Kost und Logis erfolgen. Vor allem aber nimmt die Bedeutung kleinerer Aufwendungen für die Ausübung des Sports in dem Maße zu, je niedriger die Grenze für die Brutto-Einnahmen angesiedelt wird. Anders gewendet: Je niedriger der Nettowert ist, ab dem „Einnahmen von erheblichem Umfang “ angenommen werden, umso größer wird die Bedeutung der Aufwendungen und der diese betreffenden Ermittlungen.

cc) Allgemeine Bewertung von $\$ 4$ Abs. 7

(1) Systematik

In der Literatur umstritten ist die Frage, ob es sich bei $\$ 4$ Abs. 7 um ein Tatbestandsmerkmal oder eine objektive Bedingung der Strafbarkeit handelt. ${ }^{47}$ Für die Annahme einer objektiven Strafbarkeitsbedingung spricht vor allem die systematische Stellung der Regelung in $\$ 4$ und nicht in $\$ 3$. Dies deutet darauf hin, dass der Gesetzgeber durch die Begrenzung des Adressatenkreises eine objektive Einschränkung der Strafbarkeit vornehmen wollte. ${ }^{48}$

Eines der untersuchten Verfahren wurde von der Staatsanwaltschaft jedoch nach $\mathbb{1} 170$ Abs. 2 StPO mit der Begründung eingestellt, dass „nicht sicher feststellbar“ gewesen sei, ob der Beschuldigte „die Dopingmittel zu einem Zeitpunkt eingenommen“ habe, „in dem er schon Kenntnis von seiner Aufnahme in den Testpool und damit seiner Normadressateneigenschaft hatte."

Mit Blick auf die systematische Stellung des Täterkreises wurde kritisiert, dass eine so wesentliche Voraussetzung der Strafbarkeit nicht erst im siebten Absatz der Strafvorschrift erfolgen sollte.

"Aus rechtlicher Sicht, ich meine, es gibt natürlich viele Gesetze, die noch deutlich komplexer und komplizierter aufgebaut sind, nichtsdestotrotz aus der Sicht des Anwenders oder vor allem auch des Adressaten wünscht man sich natürlich vielleicht eine deutlichere Herausstellung, Hervorhebung oder

47 Siehe hierzu Erbs/Kohlhaas/Wußler, 228. EL Januar 2020, AntiDopG $₫ 4$ Rn. 11; MüKo-StGB/Freund, 3. Aufl. 2018, AntiDopG $\$ \$$ 1-4 Rn. 113.

48 So auch die Terminologie in der Gesetzesbegründung BT-Drs. 18/4898, 31. Kritisch mit Hinweis auf die Benachrichtigungspflicht über die Zugehörigkeit eines Sportlers oder einer Sportlerin zum Testpool Erbs/Kohlhaas/Wußler, 228. EL Januar 2020, AntiDopG $\$ 4$ Rn. 11. 
ein An-den-Anfang-Stellen des jeweiligen Adressaten- und Strafrechtskreises. " $(N)$

(2) Grund für geringe Fallzahlen und seltene Anklagen

Die Beschränkung des Adressatenkreises in $\$ 4$ Abs. 7 wurde als wesentlicher Grund für die geringen Fallzahlen im Bereich des Selbstdopings gewertet.

„Also sicherlich ist die Regelung des $\$ 4$ Abs. 7 ein Grund eben dafür, dass wir nur sehr wenige Verfahren im Bereich Spitzensport haben, weil man sich ja auch klar machen muss, die Anzahl der Atbleten, die eben in den Testpool fallen, auf der einen Seite und auf der anderen Seite die Athleten, die eben tatsächlich erhebliche Einkünfte aus ibrer sportlichen Tätigkeit erlangen, die ist jetzt nicht sehr groß. "(StA 1)

Dieser Eindruck wurde durch die Aktenuntersuchung bestätigt; Beschuldigte waren häufig keine Testpool-Athleten und auch die Erzielung von erheblichen Einnahmen konnte nicht nachgewiesen werden. Eine Absenkung der in $\$ 4$ Abs. 7 formulierten Hürden könnte dazu führen, dass mehr Verfahren zur Anklage gebracht werden.

„Sobald man eben keinen Testpool-Athleten hat, dann wird der Nachweis oftmals schwierig. Da wäre, wenn man insoweit mehr Fälle haben wollte, sicherlich eine Nachjustierung sinnvoll." (StA 1)

\section{(3) Ungleichbehandlung von Sportlern}

Begründet wurde die Einschränkung in $\$ 4$ Abs. 7 mit dem Hinweis darauf, dass die Integrität des organisierten Sports allein durch das Verhalten „der in der Öffentlichkeit wahrgenommenen Leistungssportlerinnen und Leistungssportler bedroht“ werde; „reine Freizeitsportler sollen daher, selbst wenn sie an Wettkämpfen des organisierten Sports teilnehmen (z. B. größere Laufveranstaltung), nicht von der Strafnorm erfasst werden. “49

Im Schrifttum ist $\$ 4$ Abs. 7 als „willkürliche“ Einschränkung des Tatbestandes kritisiert worden, die in „keinem sachlichen Zusammenhang mit

49 BT-Drs. 18/4898, 32. 
dem Unwertgehalt der begangenen Dopingtaten" stehe. ${ }^{50}$ Der Experte der NADA äußerte sich im Interview ähnlich kritisch.

"Was wir im Austausch mit den Staatsanwaltschaften natürlich gesehen haben, ist, dass wir natürlich jetzt den Testsportathleten und denjenigen, der jetzt doch deutlich ein Einkommen aus dem Sport erzielt, in eine Situation bringen, die ibn negativ privilegiert gegenüber allen anderen Sportlerinnen und Sportlern, die ggf. an dem gleichen Event oder an der gleichen Wettkampfveranstaltung teilnehmen. Das sollte man gg. nochmals in den Fokus rücken." $(N)$

Tatsächlich hat $\$ 4$ Abs. 7 zur Folge, dass Sportler, die am selben Wettbewerb teilnehmen, vom AntiDopG unterschiedlich behandelt werden. Die in der Gesetzesbegründung beschriebene Unterscheidung zwischen Leistungs- und Freizeitsportlern mag dabei in bestimmten Wettbewerben - etwa einem städtischen Marathon, der auch dem Breitensport offensteht Sinn machen. Allerdings reicht die Einschränkung in $₫ 4$ Abs. 7 deutlich über diese Fälle hinaus. So nehmen etwa an einer Deutschen Meisterschaft in der Regel ausschließlich Leistungssportler teil; Testpool-Athleten sind jedoch nur diejenigen, die bereits den Sprung in den entsprechenden (je nach Sportart: A- oder B-Kader) geschafft haben. Obwohl die Sportler im direkten Wettbewerb miteinander stehen, soll nur derjenige bestraft werden, der bereits dem Kader angehört - nicht hingegen derjenige, der durch seine Teilnahme versucht, in den Kader aufgenommen zu werden. ${ }^{51}$

Die Ungleichbehandlung von Teilnehmern desselben sportlichen Wettbewerbs ist - gerade wenn es sich um Veranstaltungen handelt, an der üblicherweise keine Freizeitsportler teilnehmen - mit Blick auf die Ziele von Integrität und Fairness des organisierten Sports nur schwer vereinbar. Es spricht vieles dafür, den Tatbestand nicht durch personenbezogene Merkmale einzuschränken, sondern durch eine klare Begrenzung der erfassten Wettbewerbe (siehe hierzu C II 1).

50 MüKo-StGB/Freund, 3. Aufl. 2018, AntiDopG \$\$ 1-4 Rn. 112.

51 So auch Freund, der die Situation wie folgt beschreibt: „Von zwei Sportlern, die sich im Vorfeld gedopt haben und daher sportrechtswidrig an demselben Wettkampf teilnehmen, wird nur derjenige bestraft, der vielleicht gerade erst in den Kreis der ,Spitzensportler' aufgerückt ist, während sein gleichermaßen gedopter Konkurrent straffrei bleibt, wenn er nur ganz kurz davor steht, in diesen ,erlauchten' Kreis aufgenommen zu werden." Siehe dazu MüKo-StGB/Freund, 3. Aufl. 2018, AntiDopG $\$ \$ S 1-4$ Rn. 112. 


\section{B. Ergebnisse der Evaluierung}

d) Tätige Reue

aa) Legislativer Kontext

Nach $\int 4$ Abs. 8 wird wegen eines Erwerbs oder Besitzes von Dopingmitteln nach $\int 3$ Abs. 4 nicht bestraft, wer freiwillig die tatsächliche Verfügungsgewalt über das Dopingmittel aufgibt, bevor er es anwendet oder anwenden lässt. Die Vorschrift war im Regierungsentwurf noch nicht enthalten, sondern gelangte durch Beschlussempfehlung des Sportausschusses in das Gesetz. ${ }^{52}$ Als persönlicher Strafaufhebungsgrund soll die Vorschrift Straffreiheit ermöglichen, wenn der Sportler oder die Sportlerin vom Selbstdoping Abstand nimmt und freiwillig dafür sorgt, dass er oder sie mit dem Dopingmittel nicht mehr der Integrität des Sportes schaden kann. Die Straffreiheit setzt voraus, dass der Sportler oder die Sportlerin noch vor Anwendung des Dopingmittels die Verfügungsgewalt über das Dopingmittel aufgibt. ${ }^{53}$ Der Sportler oder die Sportlerin muss hierfür eine nach außen sichtbare Handlung vornehmen, die dazu führt, dass er oder sie nicht mehr über das Dopingmittel verfügen kann (Entsorgung, Übergabe an Behörde etc.). ${ }^{54}$ Neben dieser schutzzweckbezogenen Erwägung sprechen auch spezialpräventive Gründe für den Verzicht auf Strafe in solchen Fällen: In Fällen, in denen der Sportler oder die Sportlerin durch die Besitzaufgabe von Selbstdopingabsichten erkennbar Abstand nimmt, ist eine Einwirkung durch die Strafe nicht erforderlich. Konsequenterweise setzt der Strafaufhebungsgrund indes weiter voraus, dass die Aufgabe der tatsächlichen Verfügungsgewalt freiwillig erfolgt. Der Sportler oder die Sportlerin muss aus selbstgesetzten Motiven heraus die Verfügungsgewalt über die Dopingmittel aufgeben und freiwillig dafür sorgen, dass er oder sie mit dem Dopingmittel nicht mehr der Integrität des Sportes schaden kann. 55

Die Schaffung eines Strafaufhebungsgrundes der tätigen Reue wurde von der Literatur begrüßt. ${ }^{56}$

52 Vgl. dazu und zum Folgenden BT-Drs. 18/6677, S. 4, 12 f.

53 Lehner/Nolte/Putzke/Putzke, Anti-Doping-Gesetz, $\$ 4$ Rn. 94.

54 Lehner/Nolte/Putzke/Putzke, Anti-Doping-Gesetz, $\$ 4$ Rn. 94.

55 Lehner/Nolte/Putzke/Putzke, Anti-Doping-Gesetz, $\$ 4$ Rn. 95.

56 MüKo-StGB/Freund, 3. Aufl. 2018, AntiDopG $\$ \$ 1$ 1-4 Rn. 75; ferner Lehner/Nolte/ Putzke/Putzke, Anti-Doping-Gesetz, $\mathbb{4}$ Rn. 97. 
bb) Ergebnisse der Studie

In den ausgewerteten Akten spielte die Vorschrift keine Rolle. Insbesondere war kein Fall feststellbar, in dem ein Strafverfahren (teilweise) eingestellt wurde, weil sich im Zuge der Ermittlungen herausgestellt hatte, dass ein Sportler oder eine Sportlerin die Verfügungsgewalt an Dopingmitteln, die sie erworben oder besessen haben sollen, vor Beginn der Ermittlungen wieder aufgegeben hatten. Ob und in welchem Umfang die Vorschrift individuelle Bedeutung für einzelner Sportlerinnen und Sportler hat, die mit Blick auf die strafrechtliche Privilegierung Dopingmittel entsorgen und deshalb von Dopingabsichten Abstand nehmen, lässt sich indes nicht feststellen.

Aus der geringen praktischen Bedeutung der Vorschrift zur tätigen Reue lässt sich indes nicht ableiten, dass der Strafaufhebungsgrund keine kriminalpolitische Berechtigung hätte. Im Gegenteil sind durchaus Fälle vorstellbar, in denen zunächst Indizien (etwa Aussagen aus dem Umfeld, Dokumente) den Anfangsverdacht eines Erwerbes oder Besitzes von Dopingmitteln nahelegen, sich aber in Folge der Ermittlungen herausstellt, dass der Sportler bzw. die Sportlerin die Verfügungsgewalt inzwischen freiwillig aufgegeben hat. Die Fortführung der Ermittlungen oder gar eine Bestrafung ist in solchen Fällen aus den oben unter aa) genannten Schutzzwecküberlegungen und spezialpräventiven Gründen nicht erforderlich. Im Übrigen kann in dem Strafaufhebungsgrund auch ein gewisses Korrektiv zur umfassenden, nicht mengenmäßig begrenzten Erwerbs- und Besitzstrafbarkeit nach $₫ 4$ Abs. 2 gesehen werden.

\section{Erkenntnisse zu prozessualen Fragen}

a) Kenntniserlangung

aa) Wege der Kenntniserlangung

Von möglichen Fällen des Selbstdopings erfuhren die Strafverfolgungsbehörden insbesondere durch Anzeigen oder informatorische Mitteilungen der NADA $(65,0 \%)$. Die NADA stützte ihre Mitteilungen überwiegend auf das Ergebnis einer positiven Dopingprobe (56 Verfahren) oder auf anony- 


\section{B. Ergebnisse der Evaluierung}

me Hinweise über ihr BKMS-Meldesystem (9 Verfahren). ${ }^{57}$ Nach einer Meldung durch die NADA leiteten die Staatsanwaltschaften meist binnen weniger Tage Ermittlungsverfahren ein. Die Bedeutung der NADA für die Kenntniserlangung von Straftaten im Bereich des Selbstdopings bestätigte sich auch in den Interviews.

„Also gerade in dem Spitzensportbereich ist wie gesagt praktisch die NADA die einzige Quelle etwaiger Anzeigen, insofern sind die Informationen schon bedeutsam. Die kommen halt im Bereich von 20 bis 30 pro Jabr rein und sonst gibt es in dem Bereich keine Starter für irgendwelche Ermittlungen. Deswegen ist auch die Zusammenarbeit mit der NADA sehr wichtig." (StA 3)

In 25,2\% der Fälle ergab sich der Anfangsverdacht der Staatsanwaltschaften aus anderen Verfahren, insbesondere aus Ermittlungen gegen Händler von Dopingmitteln nach $₫ 2 .{ }^{58}$ Die Strafverfolgungsbehörden leiteten hier Verfahren gegen die Abnehmer der Mittel ein, deren Daten sich aus den Bestelllisten der Händler ergaben. Zwischen den Staatsanwaltschaften zeigte sich hier eine uneinheitliche Praxis: Während einige allein wegen $\mathbb{} 2$ (Erwerb) ermittelten, ${ }^{59}$ führten andere Staatsanwaltschaften die Verfahren - auch ohne dass Hinweise auf eine Teilnahme an sportlichen Wettbewerben oder die Tätereigenschaft nach $\$ 4$ Abs. 7 bestanden - wegen Selbstdopings. Diese Verfahren wurden jedoch sämtlich ohne Auflagen eingestellt.

Fünf der Verfahren wurden den Staatsanwaltschaften durch den Zoll gemeldet, zwei Verfahren durch dritte Anzeigeerstatter. Hinweise durch Sportverbände gab es im untersuchten Sample nicht.

57 Zwei weitere Verfahren wurden von der NADA selbst angestoßen aufgrund medialer Berichterstattung bzw. öffentlicher Äußerungen des Athleten.

58 Ebenfalls eine Rolle spielten Zufallsfunde bei Wohnungsdurchsuchungen oder der Telekommunikationsüberwachung.

59 Diese Verfahren gingen in die hiesige Auswertung dann nicht ein. 
Abb. 3: Ursprung des Anfangsverdachts

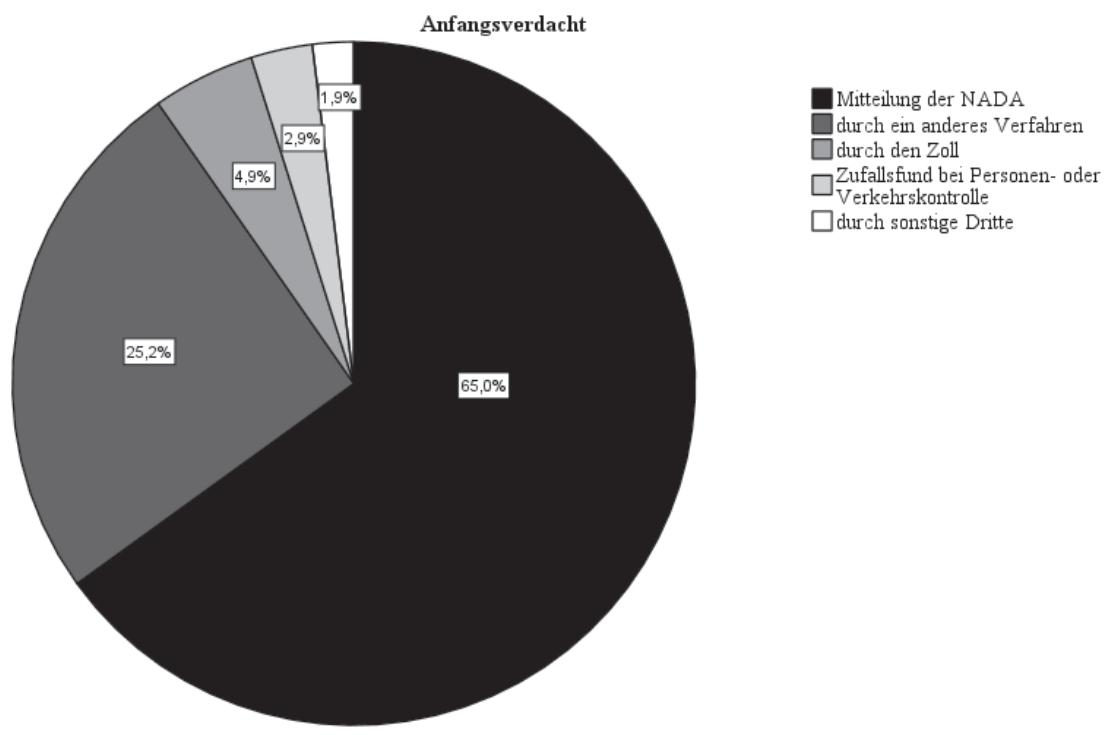

bb) Kronzeugenregelung

Im Zusammenhang mit der Kenntniserlangung von Fällen des Selbstdopings thematisierten die Interviewpersonen regelmäßig die Einführung einer Kronzeugenregelung. Eine Kronzeugenregelung für Sportler und Sportlerinnen, die des Selbstdopings beschuldigt werden, existiert bislang nicht. $\$ 46$ b I 1 StGB i.V.m. $\$ 100$ a Abs. 2 Nr. 3 StPO verweist allein auf den Qualifikationstatbestand der gewerbs- oder bandenmäßigen Begehung in $\$ 4$ Abs. 4 Nr. 2 lit. b und schließt damit den gedopten Sportler bzw. die Sportlerin vom Privileg einer Kronzeugenregelung aus. ${ }^{60}$

Angesichts der begrenzten Möglichkeiten der Kenntniserlangung sprachen sich einige der interviewten Staatsanwälte für eine Ausdehnung der Kronzeugenregelung auf den Bereich von $\$ 3$ aus. Die Aussicht auf eine Milderung oder das Absehen von Strafe könne dabei helfen, Strukturen aufzudecken und andere dopende Sportler und Sportlerinnen zu überführen.

60 Hierzu ausführlich und kritisch: Cherkeh, SpuRt 2019, S. 167, 168; Hauptmann/ Klarmann, SpuRt 2019, S. 197. 
„Also klar, ganz oben auf der Wunschliste wäre, eine Kronzeugenregelung in das Antidopinggesetz aufzunehmen, weil, wie bereits gesagt, wir gerade im Hochleistungssport keine Fälle bekommen. Ich hatte vorhin bereits gesagt, auf welchem Weg im Breitensport, im Kraftsport die Fälle kommen. Diese Möglichkeiten fallen ja praktisch alle weg und im Hochleistungssport kriegen wir keine Fälle. Entweder kommen Anzeigen aus der NADA und aus der Szene. [...] Man müsste schon den Athleten auch einen Anreiz verschaffen, sich den Strafverfolgungsbehörden gegenüber zu äußern. Zumal ja der Händler oder der Arzt die Möglichkeit hätte, sich über die allgemeine Kronzeugenregelung eine Strafrahmenmilderung zu verschaffen und der Atblet hat das einfach nicht. Das heißt, in meinen Augen ist das ein ganz wichtiges Signal an die Szene zu sagen, also die Kooperation lohnt sich." (StA 3)

Zur Einführung einer Kronzeugenregelung für dopende Sportler und Sportlerinnen siehe ausführlich unten (4.b.aa.).

\section{b) Ermittlungsmaßnahmen}

In $54,4 \%$ der Verfahren wurden keine eigenen Ermittlungen durch die Strafverfolgungsbehörden in den Akten dokumentiert, die über die Beschuldigtenvernehmung hinausgingen. Bei den durchgeführten Ermittlungsmaßnahmen handelte es sich häufig um Durchsuchungen von Wohnungen, Kraftfahrzeugen und Geschäftsräumen (38,8\%); seltener kam es zu Urin-, Blut- oder Haaranalysen (6,8 \%) und nur in je einem Fall zu einer Observation und einer Finanzermittlung. Unterhalb der Schwelle staatlicher Eingriffsmaßnahmen wurden vielfach Online-Recherchen durchgeführt, um einen Eindruck von den sportlichen Aktivitäten und potentiellen Einnahmen des Beschuldigten zu erhalten.

"Also oftmals sind es eben Durchsuchungsmaßnahmen sowobl im Breitenals auch im Spitzensport, dass eben durchsucht wird, sowobl bei dem beschuldigten Athlet zu Hause als auch teilweise dann eben in seinem Sportverband entsprechend den Räumlichkeiten." (StA 1)

„Klar, die erste Maßnahme ist natürlich die Hausdurchsuchung. Da finden wir eigentlich immer etwas; wenn ein Anfangsverdacht besteht, mit sehr groBem Erfolg." (StA 2)

In der Bereitschaft zur Durchführung von Ermittlungsmaßnahmen zeigen sich erneut erhebliche Unterschiede zwischen den Staatsanwaltschaften. 
Teilweise wurden Ermittlungsmaßnahmen zu einem sehr frühen Stadium des Verfahrens angeordnet, ohne dass die rechtlichen Voraussetzungen für ein strafbares Selbstdoping - etwa die Teilnahme an einem Wettbewerb des organisierten Sports oder die Tätereigenschaft nach $\$ 4$ Abs. 7 geprüft wurden. Es erfolgten oft aufwendige - und für den Beschuldigten invasive - Wohnungsdurchsuchungen; die Verfahren wurden jedoch, selbst beim Auffinden von Mitteln, dann mit Blick auf die fehlenden rechtlichen Voraussetzungen von $\$ 3$ eingestellt. Hier würde sich, sowohl aus Gründen der Effektivität als auch mit Blick auf einen Schutz des Beschuldigten, eine umgekehrte Vorgehensweise anbieten.

Es zeigte sich jedoch auch das entgegengesetzte Bild. Trotz bestehender Hinweise auf eine Einnahme von Dopingmitteln wurden Verfahren ohne Durchführung von Ermittlungen mit der Begründung eingestellt, dass die rechtlichen Voraussetzungen nicht vorliegen würden. Eine tatsächliche Prüfung der Tatbestandsvoraussetzungen erfolgte allerdings nicht.

Die NADA erstattete Anzeige gegen einen Dartspieler, der am Rande des German Masters positiv auf Amphetamine getestet worden war. Es handelte sich nicht um einen Testpool-Athleten. Die Staatsanwaltschaft verzichtete auf weitere Ermittlungen. Das Verfahren wurde nach $\$ 170$ Abs. 2 StPO mit der Begründung eingestellt, dass kein Hinweis auf Einnahmen von erheblichem Umfang vorliege. Etwaige Einkünfte des Athleten wurden von der Staatsanwaltschaft allerdings nicht geprüft.

In anderen Verfahren wurde auf die Vernehmung von Beschuldigten verzichtet, „da aufgrund Erfahrungen aus anderen Ermittlungsverfahren damit zu rechnen ist, dass diese von ihrem Schweigerecht Gebrauch machen werden. Das Verfahren ist daher gemäß $\$ 170$ Abs. 2 StPO einzustellen.“ (Aktenauszug)

\section{c) Abschluss der Verfahren}

Sechs der untersuchten Verfahren wurden letztlich nicht als Selbstdoping, sondern als Verstöße gegen $\$ 2$ geahndet oder nach $\$ 153$ a StPO unter Auflagen eingestellt. ${ }^{61}$ Wegen des Vorwurfs des Selbstdopings erging in drei

61 In den Verfahren stand der Vorwurf des Selbstdopings im Raum, weshalb die Verfahren in das Sample aufgenommen wurden (siehe S.4). Es ergingen im Er- 


\section{B. Ergebnisse der Evaluierung}

Fällen ein Strafbefehl ${ }^{62}$ (über 30 bzw. 40 bzw. 120 Tagessätze). Die übrigen wegen Selbstdopings geführten Verfahren wurden eingestellt; davon lediglich vier Verfahren nach $\$$ 153a StPO unter Erteilung einer Geldauflage. Eine Verurteilung wegen Selbstdopings lag in dem untersuchten Sample nicht vor.

Abb. 4: Abschluss des Strafverfahrens

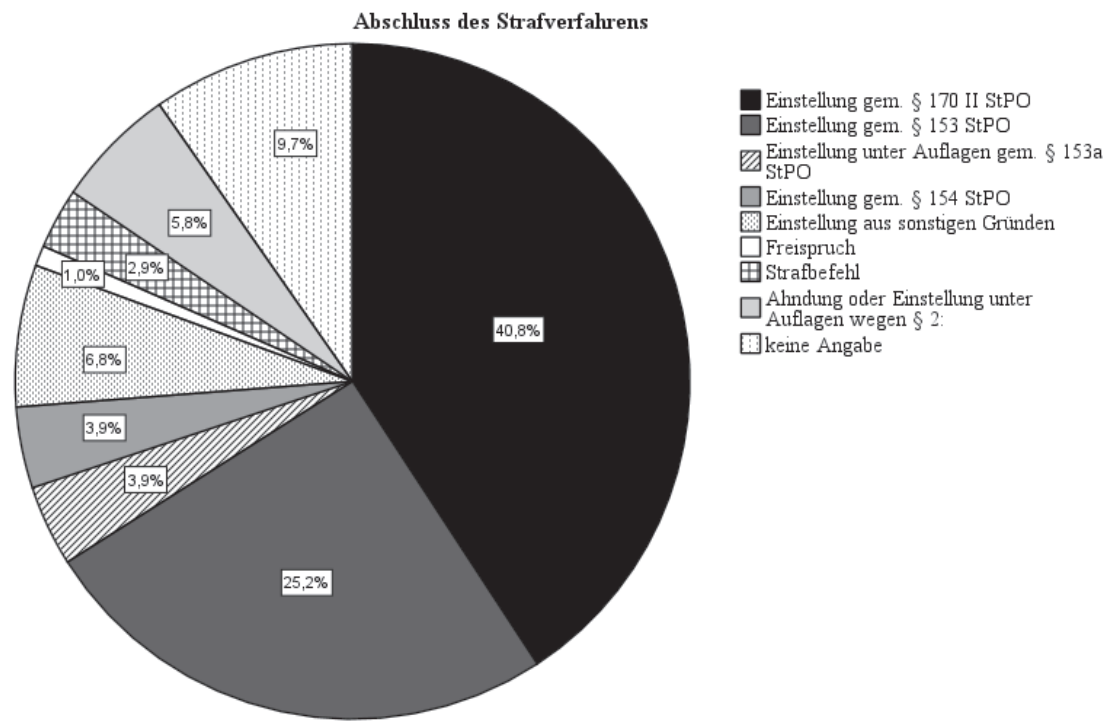

Auch in den Interviews bestätigte sich, dass Fälle des Selbstdopings bislang kaum zu den Gerichten gelangten.

R 2: $\quad$ "Also ich würde sagen, nein, da war nicht ein einziger Fall [des Selbstdopings] dabei."

Interviewer: „Dann ist das kein Tatbestand, der Sie in der Praxis beschäftigt, wo wir jetzt über die Auslegung der einzelnen Tatbestandsmerkmale sprechen könnten?"

R 2: NNee, das auf keinen Fall.“

gebnis zwei Verurteilungen, zwei Strafbefehle und zwei Einstellungen gegen Auflagen nach $₫ 153$ a StPO wegen Verstoßes gegen $\$ 2$.

62 In einem der beiden Strafbefehle werden $₫ 4$ Abs. 1 Nr. 4 und 5 benannt, in der Begründung wird allerdings ausschließlich auf $₫ 2$ Abs. 3 eingegangen. 
aa) Keine Verurteilungen wegen Selbstdopings

Die Auswertung der von den Staatsanwaltschaften zur Verfügung gestellten Verfahrensakten zeigt eine bemerkenswerte Auffälligkeit: Die Verfahren, die wegen eines Verstoßes gegen das Selbstdopingverbot geführt worden sind, wurden fast ausnahmslos nach $\$ 170$ Abs. 2, $\mathbb{1} 153$ StPO eingestellt.

Die Befunde der Aktenauswertung decken sich mit dem Ergebnis der Befragungen insoweit, als die Gesprächspartner bestätigen, dass es bislang keine Verurteilungen nach einer Hauptverhandlung wegen Verstößen gegen das Selbstdopingverbot gebe. Hinweise auf solche Urteile, die sich aus den vom Statistischen Bundesamt zur Verfügung gestellten vorläufigen Daten für 2017 und 2018 ziehen lassen, können die befragten Staatsanwälte nicht bestätigen. Stattdessen sagte ein Staatsanwalt einer Schwerpunktstaatsanwaltschaft zu zwei in seinem Zuständigkeitsbereich angeblich ergangenen Urteilen:

„Ich glaube, dass es sich um einen Fehler bei der statistischen Erfassung gehandelt hat." (StA 3)

Ein Staatsanwalt einer anderen Schwerpunktstaatsanwaltschaft teilte auf Anfrage zu einem möglicherweise in seinem Zuständigkeitsbereich ergangenen Urteil mit:

„Mir ist aktuell kein Verfahren der Schwerpunktstaatsanwaltschaft in Erinnerung, welches nach durchgeführter Hauptverhandlung bislang zu einer Verurteilung wegen Selbstdopings geführt hat." (StA 1)

Ein Richter antwortet auf die Frage, ob er rechtlichen Problemen bei der Anwendung der auf das Selbstdoping bezogenen Straftatbestände begegnet sei:

„Nein, weil nie angewendet." ( $R$ 1)

bb) Einstellungen nach $₫ 170$ Abs. 2 StPO oder $₫ 153$ StPO

Ob ein Verfahren nach $₫ 170$ Abs. 2 StPO oder $₫ 153$ StPO eingestellt wurde, hing weniger von den Einstellungsgründen als von der Praxis der je- 


\section{B. Ergebnisse der Evaluierung}

weiligen Staatsanwaltschaften ab. ${ }^{63}$ Insbesondere erfolgten Einstellungen nach $\$ 153$ StPO vielfach auch dann, wenn nach den Ermittlungsergebnissen die tatbestandlichen Voraussetzungen nicht vorlagen oder nicht nachweisbar waren. In der Begründung der Einstellungsverfügung wurde auf Zweifel an der Tatbestandsmäßigkeit nicht eingegangen, sondern auf fehlende Vorstrafen und die spezialpräventive Wirkung des Ermittlungsverfahrens hingewiesen.

„Der Beschuldigte ist bisher strafrechtlich nicht in Erscheinung getreten. Es kann erwartet werden, dass der Beschuldigte durch das bisherige Ermittlungsverfahren hinreichend beeindruckt und gewarnt ist. "64

Für die Einstellung von Verfahren wegen Selbstdopings zeigten sich in der Analyse unterschiedliche Gründe, etwa:

- Es handelte sich bei dem aufgefundenen Mittel um kein verbotenes Dopingmittel.

- Der Beschuldigte hat seinen Wohnsitz im Ausland und ein Rechtshilfeersuchen wurde nicht als erfolgsversprechend bewertet.

- Es erfolgte bereits „eine deutliche Sanktion seitens des Sportverbandes"65, die ein strafrechtliches Vorgehen nicht mehr erforderlich erscheinen lasse. ${ }^{66}$

- Das Verfahren dauerte länger als ein Jahr und wurde „unter Berücksichtigung des zwischenzeitlich eingetretenen weiteren Zeitablaufs“ eingestellt.

Der Nachweis aller Strafbarkeitsvoraussetzungen gestaltete sich in nahezu allen Verfahren als schwierig.

Nachweisprobleme bereitete insbesondere das Merkmal der „Einnahmen von erheblichem Umfang“. Die Staatsanwaltschaften stellten hier vielfach keine Ermittlungen an, sondern entschieden sich für eine Einstellung („Es kann nicht mit einem hinreichenden Grad an Gewissheit angenommen werden, dass der Beschuldigte Einnahmen erzielte.“).

63 Einige Staatsanwaltschaften stellten Verfahren grundsätzlich nicht nach $₫ 170$ Abs. 2 StPO ein, während andere ohne erkennbares Differenzierungskriterium entweder $\mathbb{1 5 3}$ oder $₫ 170$ Abs. 2 StPO wählten; wieder andere stellten nicht nach $\$ 153$ StPO ein, wenn ein Tatbestandsmerkmal nachweislich nicht vorlag.

64 Die Begründungen in den Einstellungsverfügungen nach $\mathbb{\$} 153$ StPO lesen sich hier fast wortgleich.

65 Alle folgenden Zitate sind Auszüge aus den untersuchten Akten.

66 In diesen Fällen erfolgte die Einstellung nach $\$ 153$ StPO. 
Ein weiterer Grund für die Einstellung von Verfahren war die Vorlage eines medizinischen Attestes. Eine Überprüfung des ärztlichen Attestes durch die Staatsanwaltschaften konnte den Akten nicht entnommen werden; teilweise wurde ausdrücklich darauf hingewiesen, dass die Einholung eines „kostenträchtigen Gutachtens“ „nicht im Verhältnis zur Bedeutung der Sache" stehe. Die Verfahren wurden eingestellt.

Ein besonderes Augenmerk möchten die Sachverständigen auf zwei Probleme bei der effektiven Verfolgung von Selbstdoping richten: die Einstellung wegen eines fehlenden Vorsatzes bezüglich der Einnahme von Dopingmitteln (cc) sowie die Einstellung ohne erkennbare Gründe (dd).

cc) Einstellung wegen eines fehlenden Vorsatznachweises bezüglich der Einnahme von Dopingmitteln

„Der Beschuldigte bestreitet, die ibm zur Last gelegte Tat begangen zu haben. Unmittelbare Tatzengen sind nicht vorhanden. Beweismittel, die eine bedenkenfreie Überführung ermöglichen könnten, liegen nicht vor. Nach Durchführung der umfangreichen Ermittlungen kann nicht mit der für eine Anklageerbebung erforderlichen Wabrscheinlichkeit festgestellt werden, dass der Beschuldigte die Substanz wissentlich und willentlich eingenommen hat."

Trotz des Vorliegens einer positiven Dopingprobe stellten Staatsanwaltschaften zwölf Verfahren mit der Begründung ein, dass dem Beschuldigten eine wissentliche Einnahme des Dopingmittels nicht nachzuweisen sei.

(a) Einstellungen erfolgten auch dann, wenn die Erklärung des Beschuldigten für den Nachweis der Substanz in seinem Blut oder Urin nur als fernliegend bezeichnet werden kann. So erklärte ein Radrennfahrer seine positive Dopingprobe in der Beschuldigtenvernehmung wie folgt:

„Zusammengefasst waren das Tabletten meiner an Krebs erkrankten Mutter gegen Bluthochdruck und zur Entwässerung. Meine Mutter wollte diese Tabletten in einen Dosierer bzw. Spender geben. Dabei sind aber ein paar Tabletten zu Boden gefallen. Ich habe eine dieser Tabletten aus dem Spender genommen und die anderen vom Boden gemampft. (...) Meine Mutter hat mir erst später erklärt, was das für Tabletten sind und welchen Wirkstoff sie haben. Das war Freitag vor dem Rennen." 
Der Staatsanwaltschaft genügt diese Einlassung für eine Einstellung nach \170 Abs. 2 StPO:

„Der Beschuldigte hat die ihm zur Last gelegte Tat bestritten und sich dahin eingelassen, er nehme 'so einen Scheiß' nicht und könne sich den Nachweis der Substanzen Amphetamin, Heptaminol und Hydrochlorothiazid in seinem Blut nicht erklären. Weitere objektive Beweismittel, welche zur Überführung des Beschuldigten geeignet sind, stehen nicht zur Verfügung. Bei dieser Sach- und Beweislage hat eine Erhebung der öffentlichen Klage keine Aussicht auf Erfolg, da mit einer Verurteilung des Beschuldigten angesichts des im Strafprozess geltenden Grundsatzes, dass bei verbleibenden Zweifeln stets zu Gunsten des Beschuldigten zu entscheiden ist, nicht zu rechnen ist."

(b) Mehrfach wurde von Beschuldigten vorgebracht, dass der Nachweis verbotener Substanzen auf verunreinigte Lebensmittel oder Trinkwasser zurückzuführen sei. Ein Verfahren soll hier exemplarisch skizziert werden.

Bei dem Beschuldigten handelte es sich um einen Testpool-Athleten. Im Rahmen einer unangekündigten Trainingskontrolle der NADA wurde im Urin des Athleten, einem Bahnradfahrer, Tamoxifen nachgewiesen. ${ }^{67}$ Die NADA brachte den Sachverhalt zur Anzeige. Der Beschuldigte bestritt die Einnahme und gab eine eidesstattliche Versicherung ab, nie wissentlich Dopingmittel zu sich genommen zu haben. Er gab an, dass der Stoff allenfalls durch verunreinigtes Trinkwasser in seinen Körper gelangt sein könnte, und stellte Zeitungsartikel zur Verfügung, die über eine Verunreinigung von französischem Trinkwasser durch Tamoxifen berichteten. Darüber hinaus erbrachte er den Nachweis, dass er kurz vor der Trainingskontrolle durch die NADA in einem belgischen Trainingscamp war. Der Trainer hatte noch die (teilweise angebrochenen) Wasserflaschen aufgehoben, die die Sportler im Trainingscamp benutzt hatten. Das Wasser aus diesen Flaschen wurde getestet - es konnte keine Menge an Tamoxifen darin nachgewiesen werden, die den positiven DopingTest erklärt hätte.

Die Staatsanwaltschaft stellte hierzu zusammenfassend fest:

„Die Mutmaßung des Beschuldigten, wonach er den Wirkstoff Tamoxifen unwissentlich über Nahrungsergänzungsmittel oder über verunreinigtes Wasser zu

67 Tamoxifen wird zum Doping verwendet, um Nebenwirkungen einer AnabolikaEinnahme (z.B. Brustwachstum bei Männern) entgegenzuwirken. Da Anabolika aber in der Regel schneller abgebaut werden als Tamoxifen, ist oftmals nur dieser Stoff nachweisbar. 
sich genommen haben will, ist in Anbetracht der durchgeführten chemischen Untersuchung fernliegend. Diesbezüglich war auch zu berücksichtigen, dass keiner der weiteren Sportler des Teams X in der Folge des Trainingsaufenthaltes in Belgien positiv auf den Wirkstoff Tamoxifen getestet wurde. Der NADA sind auch keine Fälle bekannt, in denen es zu Dopingfällen mit der Substanz Tamoxifen aufgrund von verunreinigtem (Trink-)Wasser gekommen ist. "

Gleichwohl stellte die Staatsanwaltschaft das Verfahren nach $\$ 170$ Abs. 2 StPO ein.

„Die durchgeführten strafprozessualen Maßnahmen haben hingegen neben dem positiven Dopingbefund keine weiteren Beweise erbracht, die den Schluss zuließen, dass der Beschuldigte das Dopingmittel wissentlich konsumiert oder sich hat wissentlich zuführen lassen. [...] Die Einlassung des Beschuldigten, wonach er die Substanz Tamoxifen zu keinem Zeitpunkt wissentlich bzw. absichtlich zur verbotenen Leistungssteigerung eingenommen habe, [ist daher] nicht mit der zur Anklageerhebung erforderlichen Sicherheit zu widerlegen. Insoweit war zu berücksichtigen, dass es im Profi-Radsport nicht ausgeschlossen ist, dass jemand aus dem Umfeld des Beschuldigten diesem das Mittel obne das Wissen des Beschuldigten beibrachte, um seine Leistung zu fördern, oder um ihm zu schaden. Damit ist der subjektive Tatbestand nicht nachweisbar."

Die Generalstaatsanwaltschaft kommentiert die Einstellung des Verfahrens wie folgt:

„Der zutreffende Bericht der Staatsanwaltschaft verdeutlicht, dass trotz der Erweiterung der Strafbarkeit im Bereich des Dopings auf das sogenannte Selbstdoping ein der Strafprozessordnung genügender Tatnachweise im Einzelfall schwierig bleiben wird. In den Fällen, in denen ein unmittelbarer Tatnachweis, etwa durch Beweissicherung bei Durchsuchungen oder Zeugenaussagen aus dem nahen Umfeld des Beschuldigten, nicht möglich ist, wird trotz positiver Blutproben eine Abndung vermutlich auch zukünftig häufig scheitern."

(c) Die Staatsanwaltschaften stellen hier mit Blick auf die subjektive Tatseite außergewöhnlich hohe Anforderungen an die Annahme eines hinreichenden Tatverdachts. In den untersuchten Verfahren drängte sich der Eindruck auf, dass es sich bei den Einlassungen der Beschuldigten um Schutzbehauptungen handelte. Dies gilt nicht nur für erkennbar abseitige Erklärungen - wie in Fall (a) -, sondern auch für pauschale Hinweise auf eine Verunreinigung konsumierter Lebensmittel, die durch keinerlei Untersuchungen und praktische Erfahrungen bestätigt werden (im Gegenteil: 


\section{B. Ergebnisse der Evaluierung}

in Fall (b) kann die Behauptung, das Dopingmittel sei durch verunreinigtes Trinkwasser aufgenommen worden, als widerlegt angesehen werden).

Die hier beobachtete Praxis liefe darauf hinaus, dass Verfahren wegen Selbstdopings selbst bei Vorliegen einer positiven Dopingprobe faktisch nicht zur Anklage gebracht werden können, ${ }^{68}$ sobald der Beschuldigte die Tat bestreitet. Dies gilt insbesondere dann, wenn man der Argumentation der Staatsanwaltschaft in Fall (b) folgt, der zufolge bereits die abstrakte Möglichkeit von Manipulationen durch Dritte ausreichen soll, um eine wissentliche Einnahme der Dopingmittel durch den Sportler oder die Sportlerin selbst in dubio pro reo zu verneinen.

Dass ein solcher Umgang mit der Berufung auf verunreinigte Lebensmittel keinesfalls zwingend ist, zeigt die Verurteilung des Boxsportlers Felix Sturm durch das Landgericht Köln wegen Selbstdopings (auf das Selbstdoping in Tateinheit mit der hierdurch begangenen Körperverletzung entfiel eine Freiheitsstrafe von sechs Monaten). Die 8. Strafkammer des Landgerichts Köln hatte die Eröffnung des Verfahrens zunächst mit der Begründung abgelehnt, ${ }^{69}$ dass „nach Aktenlage auch am Ende einer Hauptverhandlung ausgehend von den eingeholten sachverständigen Stellungnahmen die realistische Möglichkeit einer unvorsätzlichen Einnahme des Wirkstoffes bestehen bleibe, sodass nicht mit einer Verurteilung wegen Verstößen gegen das AntiDopG zu rechnen sei. “70 Das OLG Köln hob den Beschluss jedoch auf und ließ die Anklage gegen Sturm vor einer anderen Strafkammer zu. Die 12. Strafkammer des Landgerichts setzte sich ausführlich mit der Frage auseinander, ob das Dopingmittel Stanozolol ${ }^{71}$ vom Sportler unwissentlich aufgenommen worden sein könnte. Auf Basis von zwei Sachverständigengutachten schloss die Kammer sowohl die Fremdeinwirkungshypothese - es wurde vorgebracht, dass Mitglieder des gegnerischen Teams verunreinigte Wasserflaschen nach dem Kampf in die Kabi-

68 Dopingmittel in der Wohnung eines Testpoolathleten Tage oder Wochen nach erfolgter Kontrolle zu finden, erscheint wenig aussichtsreich. Das gleiche gilt für Zeugen, zumal hier mit der Begründung der Staatsanwaltschaft ein Belastungswille nicht ausgeschlossen werden könnte.

69 Beschluss der 8. großen Strafkammer des Landgerichts Köln vom 10.01.2019 - 108 KLs 17/18. Dazu Kubiciel, SpuRt 2020, 306 ff.

70 Zitiert nach dem Beschluss des OLG Köln vom 04.04.2019 - 2 Ws 122/19. Dazu Lorenz/Bade, JR 2020, 324.

71 Bei Stanozolol handelt es sich um ein exogen anaboles Steroid, das - wenn es über eine gewisse Dauer, etwa im Zuge der Wettkampfvorbereitung, eingenommen wird - den Aufbau von Muskelmasse mit dem Ziel eines Zuwachses an Maximal- und Schnellkraft begünstigt. 
ne gebracht hätten - als auch die Einnahme durch verunreinigte Lebensmittel und Nahrungsergänzungsmittel aus. ${ }^{72}$ Für die bewusste Anwendung des Dopingmittels sprachen die Bedeutung von Stanozolol als eines der "beliebtesten Wettkampfsteroide“, das „typischerweise im Boxsport“ eingesetzt wird, ${ }^{73}$ sowie die persönlichen und beruflichen Umstände des Athleten. Der Pressesprecher des Landgerichts wies zu Recht darauf hin, dass hier - wie auch in anderen Verfahren - der Beweis über Indizien erbracht werden musste und konnte:

„Wir haben in solchen Fällen einen ganz normalen Indizienbeweis. Und hier hat das Gericht die Einlassung des Angeklagten auch auf Grundlage der Sachverständigengutachten als Schutzbehauptung gewertet."

Das in Köln betriebene Verfahren war - bereits durch die Bestellung von zwei Sachverständigen und der umfassenden Würdigung ihrer Gutachten - aufwendig. Ob das Urteil künftig Staatsanwaltschaften und Gerichte auch in Verfahren mit weniger prominenten Beschuldigten - zu einer intensiveren Beweiserhebung und -würdigung veranlassen wird, bleibt daher abzuwarten.

dd) Einstellung ohne erkennbare Gründe

Einstellungen erfolgten in vielen Verfahren bereits bei ersten Nachweisschwierigkeiten. In einigen Fällen zeigte sich allerdings deutlich, dass von Seiten der Ermittlungsbehörden kein Verfolgungswille bestand. Beispielhaft sollen zwei Verfahren skizziert werden.

Der Beschuldigte - ein Marathonläufer - wird über das anonyme Hinweissystem der NADA gemeldet. Eine Auswertung seines Facebook-Profils durch die Staatsanwaltschaft ergibt, dass er sich wiederholt öffentlich über Dopingmittel und auch deren (Eigen-)Konsum äußert. Gegenüber Vereinsmitgliedern habe er, so der anonyme Hinweisgeber, ,zu jeder angemessenen und unangemessenen Zeit damit geprablt", Dopingmittel zu konsumieren.

72 Die Gutachten konnten belegen, dass die Mittel nicht kurz nach dem Wettkampf eingenommen worden waren; zudem wurde nachgewiesen, dass weder Fleisch noch die von dem Angeklagten verwendeten Nahrungsergänzungsmittel mit Stanozolol verunreinigt gewesen sein konnten.

73 Beschluss des OLG Köln vom 04.04.2019 - 2 Ws 122/19 Rn. 28. 


\section{B. Ergebnisse der Evaluierung}

Die Staatsanwaltschaft entscheidet zunächst, kein Verfahren zu eröffnen, da die Angaben eines anonymen Tippgebers nicht überprüfbar seien. Die NADA rügt das Vorgehen der Staatsanwaltschaft und weist darauf hin, dass der Tippgeber bereit ist, namentlich auszusagen. Daraufhin wird das Verfahren eingeleitet, allerdings kurze Zeit später nach $₫ 170$ Abs. 2 StPO eingestellt. Die Staatsanwaltschaft begründet die Einstellung damit, dass eine „Aussage-gegen-Aussage“-Konstellation vorliege. Die NADA wendet sich erneut an die Staatsanwaltschaft und kritisiert, dass die bestehenden Beweismittel nicht hinreichend gewürdigt worden seien. Daraufhin wird das Verfahren erneut aufgenommen.

Zwei Monate später wird das Verfahren erneut gem. $₫ 170$ Abs. 2 StPO eingestellt; dieses Mal mit dem Hinweis darauf, dass der Sportler an Wettbewerben im Ausland teilnahm und deutsches Strafrecht hier nicht anwendbar sei.

In der Akte findet sich zur Frage der Anwendbarkeit des AntiDopG keine rechtliche Prüfung. Das Ergebnis wäre auch nicht begründbar; schließlich ist Bezugspunkt der Tathandlung nach $\mathbb{} 3$ Abs. 1 die Anwendung des Mittels und nicht die Teilnahme an einem Wettbewerb. Für Taten nach $₫ 3$ Abs. 2 hätte die Staatsanwaltschaft die Voraussetzungen von $\$ 7$ StGB prüfen müssen.

Gegen einen Anbieter wurde wegen des Versandes von Dopingmitteln ermittelt. Der Zoll erhält eine Liste mit Bestellern und kann ein an den Beschuldigten adressiertes Paket abfangen. In dem Paket befindet sich eine Vielzahl illegaler Substanzen. Der Zoll leitet den Fund an die Staatsanwaltschaft weiter. Zudem wird die Staatsanwaltschaft darüber informiert, dass es sich bei dem Empfänger um einen Triathleten handelt, der an nationalen wie internationalen Wettbewerben teilnimmt und, was seine Homepage zeigt, durch verschiedene Sponsoren unterstützt wird. Das Verfahren wird von der Staatsanwaltschaft nach 4 Wochen gem. $\$ 170$ Abs. 2 StPO eingestellt, ohne dass Ermittlungsmaßnahmen eingeleitet wurden. Eine Begründung für die Einstellung lässt sich der Akte nicht entnehmen.

Die Lieferung des Dopingmittels an den Empfänger sowie die Hinweise auf seine Teilnahme an sportlichen Wettbewerben sowie die hieraus erzielten Einnahmen hätten zumindest Anlass für Ermittlungen geben müssen. 
Bemerkenswert erscheint auch, dass beide Staatsanwaltschaften (in Fall a und Fall b) sämtliche Verfahren, die bei ihnen wegen Selbstdopings geführt wurden, nach $\$ 170$ Abs. 2 StPO eingestellt haben.

\section{d) Strafbefehle und Strafzumessung}

Die den Sachverständigen zur Verfügung gestellten einschlägigen Verfahrensakten enthielten hinsichtlich des Selbstdopings lediglich drei Strafbefehle sowie ein freisprechendes Urteil, das nach Einspruch gegen einen Strafbefehl erging. Im letztgenannten Fall war ein Bundesliga-Ringer vom Vorwurf einer Straftat nach $₫ 4$ Abs. 1 Nr. 4 i.V.m. Abs. 7 Nr. 1 freigesprochen worden.

Zum einen fällt auf, dass zwei der Strafbefehle gegen Beschuldigte ergingen, die sich geständig eingelassen hatten. Bemerkenswert ist zum anderen, dass sich zwei in den Strafbefehlen ausgesprochene Strafen am untersten Rand des Strafrahmens bewegen: Im Fall eines Baseball-Spielers wurde wegen einer Straftat nach $\$ 4$ Abs. 1 Nr. 5 i.V.m. Abs. 7 Nr. 2 eine Geldstrafe von 40 Tagessätzen festgesetzt. Im Verfahren gegen einen Radsportler wurde wegen einer Straftat nach $₫ 4$ Abs. 1 Nr.3, Nr. 5, Abs. 7 Nr. 1 eine Geldstrafe von 30 Tagessätzen verhängt. Im Verfahren gegen einen Berufsboxer, der bei einem Wettkampf im Ausland positiv getestet worden ist, erging ein Strafbefehl über insgesamt 120 Tagessätze: 80 Tagessätze für einen „Verstoß gegen $₫ 3$ Abs. 1 S. 1“ sowie 60 Tagessätzen wegen eines „Verstoßes gegen $\$ 3$ Abs. 4 “. ${ }^{74}$ Gegen den Strafbefehl legte der Beschuldigte zunächst einen (nicht näher begründeten) Einspruch ein; dieser wurde aber am Tag der Hauptverhandlung ohne Einlassung in der Sache zurückgezogen.

Nicht ausgewertet werden konnte die Verfahrensakte des Ende April vom Landgericht Köln verurteilten Box-Profis Felix Sturm, da das Verfahren zum Zeitpunkt der Evaluation noch nicht abgeschlossen war. Von der Gesamtfreiheitsstrafe von drei Jahren entfielen auf die tateinheitlich begangenen Straftaten nach $₫ 4$ Abs. 1 Nr. 4 und 5 sowie eine damit im Zusammenhang stehende Körperverletzung eine Teilstrafe von sechs Monaten. Die schriftlichen Urteilsgründe lagen zum Zeitpunkt der Abfassung dieses Berichtes noch nicht vor.

74 Weshalb im Strafbefehl nicht die Summe von 80 Tagessätzen und 60 Tagessätzen (140), sondern 120 Tagessätze festgesetzt worden sind, ging aus den Akten nicht hervor. 
4. Bewertung

a) Allgemeine Bewertung der strafrechtlichen Regelung von Selbstdoping

aa) Überblick

Die während des Gesetzgebungsverfahrens gegen die Einführung des AntiDopG im Allgemeinen und das strafbewehrte Verbot des Selbstdopings im Besonderen vorgetragenen Bedenken haben sich teilweise aufgelöst. Auffällig ist, dass die Haltung der Athletenvertreterinnen und -vertreter positiver ist als sie es im Gesetzgebungsverfahren war. Weiterhin zeigt sich, dass die Mehrheit der befragten Staatsanwälte keine Bedenken gegen die Kriminalisierung hegt, während die befragten Richter die Tatbestände kritischer sehen; allerdings haben die Richter bislang keine Erfahrung mit Anklagen wegen strafbarer Verstöße gegen das Selbstdopingverbot sammeln können.

bb) Zur Angemessenheit der Kriminalisierung des Selbstdopings

Im Gesetzgebungsverfahren ist des Öfteren bemängelt worden, dass Fairness und sportliche Chancengleichheit zwar sportethische Werte, ${ }^{75}$ aber keine legitimen Schutzobjekte von Straftatbeständen seien. ${ }^{76}$ Bei einer Athletenvertreterin klingen diese Bedenken noch an:

„Ich finde das ein wenig schwierig, weil ja auch die Integrität des Sports für mich ein sehr hohes Gut ist. Aber es wurde ja auch mit dem AntiDopG überhaupt erst eingeführt. Ich weiß nicht, ob das die richtige Stelle ist, das Selbstdoping nochmals so sebr zu bestrafen, weil es für mich als Sportlerin eine viel höhere Strafe ist, dass man quasi zwei Jahre aus seinem Sport gesperrt wird plus alle Einnahmen aus der Sporthilfe zurückzablen muss. Das ist alles schon ein Rieseneinschnitt im Leben, den man auf gar keinen Fall auf sich nehmen will. (...) Deswegen fände ich es ein wenig kritisch zu sagen, dass man Selbstdoping in einem nationalen Gesetz bestraft, weil es eben schon anderweitig sebr hoch bestraft wird. Das ist wahrscheinlich nicht der Weg, wie man das verbindern kann." (A1)

75 DOSB, Stellungnahme zum Referentenentwurf eines Gesetzes zur Bekämpfung von Doping im Sport, S. 4.

76 Stellungnahme des Deutschen Richterbunds zum Referentenentwurf eines Gesetzes zur Bekämpfung von Doping im Sport, Nr. 7/15. 
Dennoch antwortete sie auf die Frage, ob die Strafverfolgung der unerlaubten Anwendung von Dopingmethoden und -mitteln sinnvoll sei, wie folgt:

„Ich meine, dass es generell sinnvoll ist, (...) auch aus gesundheitlichen Aspekten, gerade aus dem Aspekt, wie sich unsere Leistungsgesellschaft weiterentwickelt. Da besteht ja auch ein hohes Risiko, wenn jetzt junge Sportler das in ibrem Verein mitbekommen, auch im Freizeitbereich wird viel gedopt. (...) Deswegen finde ich es generell nicht schlecht, dass das verfolgt wird. Man muss halt die Rabmenbedingungen immer so klären, dass es verbältnismäßig ist. (A1)

Ein anderer Athletenvertreter zeigte sich im Ergebnis ebenfalls davon überzeugt, dass die Kriminalisierung sinnvoll sei:

"Ich tue mich ein bisschen schwer mit der Antwort, weil persönlich war ich immer sehr zwiegespalten, ob das der richtige Weg ist, und hatte eben Bedenken, dass diese Kriminalisierung stattfindet, auch weil ich eben viele Situationen sehe, wo junge Menschen in ein System geraten, Abhängigkeiten entstehen, Drucksituationen entstehen. Ich will nicht sagen, dass sie deswegen nicht schuldig sind, wenn sie dopen, aber dass es doch ein System bisweilen gibt, das das sehr begünstigt und das sie eben in eine sehr schwierige Situation auch bringt, wenn sie vielleicht dann nicht mitdopen, weil das auch z.T. in einer Gruppe geschieht. Deshalb war ich teilweise skeptisch, ob man diese Athleten tatsächlich noch mit einer Strafe belegen sollte, weil sie sowieso schon eine harte Strafe erdulden müssen, weil sie eigentlich ihre Karriere verlieren. Daher rührt so ein bisschen mein Zwiespalt. Im Grunde glaube ich aber schon, dass harte Mittel auch notwendig sind, weil wir ja doch sehen, dass, wenn jemand gewillt ist $z u$ dopen, es sehr schwer ist, die Leute dann auch aufzuhalten mit den Mitteln, die wir jetzt bei der Verfolgung beim An-



Auch die Mehrheit der befragten Staatsanwälte äußerte keine Zweifel an der Sinnhaftigkeit der Kriminalisierung und der Strafverfolgung von Selbstdoping.

„Also ich halte ihn durchaus für einen sehr wichtigen Tatbestand." (StA 1)

„Dass Personen, die eben mit Dopingmitteln arbeiten, mehr Trainingsfortschritt haben, mehr Erfolg haben, halte ich für ziemlich kontraproduktiv, was den Gedanken des Sports angeht. Und deswegen denke ich, dass eine prinzipielle Strafbarkeit solcher Sachverbalte sinnvoll ist, ja." (StA 5) 
Ein Staatsanwalt meldete hingegen Bedenken an:

„Ich denke, dass jeder selbstverantwortlich entscheiden muss, ob er Dopingmittel nimmt oder nicht. Gerade, wenn es z.B. darum geht - da hatte ich letztens einen Fall, da sollte jemand aus Georgien ausgeliefert werden, der mit Haftbefehl gesucht wird (die Georgier kennen dieses AntiDopG ja gar nicht und haben auch kein ähnliches Gesetz und alle sind immer überrascht, dass das ja bei uns strafbar ist). Wir gehen da wahrscheinlich so einen Sonderweg und ich meine, die Selbstverantwortung unter Sportlern dürfte ausreichen um zu sagen, nimmt man Dopingmittel oder nimmt man keine. Besitz von Dopingmitteln zum Eigengebrauch - ja, da weiß ich nicht, ob das unbedingt strafbar sein muss." (StA 2)

Allerdings hatte der Staatsanwalt nach eigenen Angaben bislang nur mit einem Fall von Selbstdoping zu tun. Gleiches gilt für die beiden Richter, die sich ebenfalls kritisch äußerten.

„Mit $\$ 3$ hatte ich ja noch nie etwas zu tun. Eine fundierte Meinung zu $\$ 3$ habe ich eigentlich noch nicht entwickelt, weil ich ibn noch nie selbst anwenden musste. Meine Meinung dazu ist ziemlich oberflächlich. $\$ 3$ halte ich ja weitgehend für überflüssig. (...) Dass sich Spitzensportler dopen oder dopen lassen, finde ich aus Sicht der Strafverfolgung völlig uninteressant. Ich wüsste nicht, warum man das nicht zulassen sollte. (...) Da habe ich meine Zweifel, weil es in erster Linie um Selbstgefährdung geht. Der einzige Grund für mich, dass man diesem Phänomen sein Augenmerk widmet, ist der Umstand, dass es ja auch minderjährige Sportler gibt, die auf alle Fälle geschützt werden müssen." ( $R$ 1)

Ein anderer gab zu bedenken:

„Warum soll ich einen Sportler davon abhalten? Ich meine, ich halte ja auch keinen davon ab, jeden Tag $10 \mathrm{~kg}$ Rindfleisch zu essen oder Schweinefleisch, und sich da irgendwie zu schaden. Also ist jetzt ein blödes Beispiel. Aber im Grunde genommen, also wenn die Selbstschädigung so in den Vordergrund tritt. Also ich würde sagen, das ist der Kern des Problems, ob man das wirklich macht oder ob man das lässt. Zumal, sagen wir mal so. Ich könnte doch besser damit leben, wenn der eigentliche Sinn, die eigentliche Sinngebung da den Sport, und zwar jetzt nicht... Doping, da würden die sagen, das ist auch Sport. Aber wenn ich das jetzt mal ausklammere, dass man da dieses mit Sicherheit bestehende Dopingproblem auf diese Art und Weise zumindest mithilft, einzuschränken. Aber da warte ich jetzt tatsächlich noch auf den ersten Fall und das ist schon ein bisschen merkwürdig, weil das kann ja fast nicht sein, wenn man genau guckt." ( $R$ 2) 
cc) Zur Angst vor vorsätzlichen Falschbeschuldigungen

Im Gesetzgebungsverfahren ist von Athletenvertretern die Befürchtung geäußert worden, Athleten könnten, beispielsweise von Konkurrenten, gezielt falsch verdächtigt werden, oder ihnen könnte eine geringe Menge Dopingmittel untergeschoben werden. Bislang ist Athletenvertreterinnen und Athletenvertretern indes kein entsprechender Fall bekannt geworden:

"Nein, ich habe kein illustratives Beispiel dafür und würde mir auch jetzt nicht die Aussage zutrauen, dass es die Athleten verunsichert hat." (A 2)

„Mir ist jetzt kein Fall bekannt." (A1)

Auf eine mögliche Verunsicherung der Athleten angesprochen, antwortete eine Vertreterin von Athleten:

„Nein, dafür habe ich kein Beispiel. (...). Aber das heißt ja trotzdem nicht, dass es o.k. ist. Es ist nur in der Wahrnehmung - glaube ich - nicht ganz so schlimm." (A1)

dd) Zur Sorge um die Bedeutung der Sportgerichtsbarkeit

Schließlich ergab die empirische Untersuchung auch keine Anhaltspunkte dafür, dass die Kriminalisierung des Selbstdopings und die staatliche Strafverfolgung zu einer Schwächung oder Entwertung der sportrechtlichen Verfahren geführt hat. Dazu sagte eine Vertreterin eines Verbandes:

„Es stimmt, dass wir das tatsächlich immer im Gesetzgebungsverfahren vorgebracht hatten und dass genau diese Schwächung auch die größte Sorge war. $\mathrm{Ob}$ das wirklich berechtigt war, können wir noch nicht so klar beantworten, weil wir noch keinen wirklichen Fall in Deutschland sehen, der unabhängig vom Breitensport so ein prominenter Fall wäre, und mit entsprechenden Strafen und Sanktionen, die das Antidopinggesetz hier vorsiebt, ausgeurteilt wäre, dass wir das überhaupt vergleichen könnten, also wo wir sagen würden, es gäbe wirklich eine Diskrepanz zwischen dem sportrechtlichen Sanktionssystem und dem strafrechtlichen. (D)

Die Athletenvertreter betonten sogar, dass die Angst vor sportrechtlichen Konsequenzen größer sei als die Angst vor Strafverfolgung. ${ }^{77}$

77 Siehe Zitate von A 1 auf S. 37 und 68. 
ee) Generelle Bewertung

Auf die Frage, ob das AntiDopG mit seinem Verbot des Selbstdopings geglückt sei, antworteten die befragten Athleten und Staatsanwälte in der Mehrzahl mit einer grundsätzlich positiven Tendenz. Beispielhaft:

„Ich glaube, insgesamt ist es schon geglückt. Ich meine eben, dass wir wirklich davon wegkommen müssen zu sagen, das AntiDopG löst die Probleme. (A1)

„Ja, ich denke, insgesamt ist es schon geglückt. Die Fälle im Spitzensport halten sich ja bisher noch in Grenzen, wenn ich das richtig verstanden habe, aber ich glaube schon, dass es insgesamt geglückt ist. "(A2)

„Es ist durchaus geglückt, einen wirklich sehr schwierigen und sehr, sehr kontrovers diskutierten Bereich strafrechtlich oder auch gesetzlich zu legitimieren. Das ist gut, das ist der richtige Weg. Natürlich ist es auch wichtig, dass man sich regelmäßig dieses Gesetz anschaut und entsprechende Nuancen verbessert." $(N)$

„Gelungen und keine Änderungswünsche. “(StA 4)

Indes werden diese positiven Aussagen von einigen mit Blick auf die geringe Anzahl von Fällen eingeschränkt:

„Also ich finde, das AntiDopG ist schon geglückt. Man kann jetzt nicht sagen, dass der Schuss nach hinten losgegangen ist. Es hat eine Vielzahl von positiven Erscheinungen mit sich gebracht. (...) Das sind alles Punkte, die die Antidopingverfolgung unterstützen, wenngleich sich tatsächlich an der ganzen Grundtendenz, dass man im Leistungssport keine Fälle bekommt durch das AntiDopG, sich die Lage nicht verbessert. (...) Aber wir haben einfach schlicht und ergreifend die Fälle nicht, denn da könnte das AntiDopG nämlich einen Anreiz oder die Möglichkeit bieten, die Fälle so zu bekommen, indem man halt dort ansetzt, wo die Informationen herkommen können. In meinen Augen kann es nur aus der Szene kommen und deswegen ist dieses Nichtvorhandensein einer sportspezifischen Kronzeugenregelung doch ein erhebliches Manko, es hat halt erheblichen Verbesserungsbedarf meines Erachtens." (StA 3)

„Ich glaube, das ist irgendwie total schwierig, das für uns zu bewerten, weil wir finden, dass es dafür zu wenige relevante Fälle gibt, an denen man etwas festmachen könnte, ob es wirkt. Wenn man jetzt - ich meine, das ist nicht wünschenswert insgesamt - aber wenn man jetzt mal so zehn, zwanzig richtige Anwendungsfälle hätte, woran man das einfach mal testet - das 
klingt ein bisschen komisch - aber woran das Gesetz wirklich einmal sozusagen durchgespielt werden würde, und damit meine ich jetzt nicht irgendwelche Bodybuilder aus irgendeinem $x$-beliebigen Studio, sondern wirklich diese Spitzensportlerinnen und Spitzensportler, um die es geht. Natürlich wünschen wir uns das nicht, völlig klar. Aber erst dann könnten wir, glaube ich, sagen: Es hat sich wirklich bewährt oder nicht." $(D)$

b) Vorschläge aus der Praxis zur Reform der strafrechtlichen Regelungen zum Selbstdoping

Trotz der grundsätzlich positiven Bewertung des Selbstdopingverbots durch die Mehrzahl der Befragten, unterbreiteten die Experten an verschiedenen Stellen Vorschläge für eine Verbesserung der strafrechtlichen Ahndung von Verstößen gegen das AntiDopG. In Bezug auf das materielle Recht stand dabei die Überarbeitung von $\$ 4$ Abs. 7 im Vordergrund, der insbesondere mit Blick auf die „Einnahmen von erheblichem Umfang“ als zu unbestimmt bewertet wurde (siehe hierzu oben 2.c.bb.). Am häufigsten wiesen die Experten jedoch auf die Einführung einer Kronzeugenregelung sowie einen besseren Schutz von Whistleblowern hin.

aa) Einführung einer speziellen Kronzeugenregelung

Wie bereits dargelegt (siehe oben 3.a.bb.), findet die Kronzeugenregelung in $₫ 46 \mathrm{~b}$ StGB auf den selbstdopenden Sportler bzw. die Sportlerin keine Anwendung. Eine eigenständige Kronzeugenregelung wie in $\$ 31 \mathrm{BtMG}$ existiert im AntiDopG bislang nicht. Für im Strafverfahren beschuldigte Sportler und Sportlerinnen besteht daher kein gesetzlicher Anreiz, eigenes Wissen über Strukturen, Hintermänner oder weitere Täter preiszugeben. Da das Selbstdoping nicht mit einer Mindeststrafe bedroht ist, haben die Staatsanwaltschaften allerdings bereits nach geltendem Recht die Möglichkeit, Verfahren im Falle einer entsprechenden Aussagebereitschaft aus Opportunitätsgründen einzustellen. Ebenso liegt es nahe, dass Gerichte eine Aufklärungshilfe im Rahmen ihrer Strafzumessungserwägungen berücksichtigen würden.

In ihren Stellungnahmen haben die NADA, der DOSB, der DAV und Athletenverbände die Einführung einer Kronzeugenregelung für Sportler 


\section{B. Ergebnisse der Evaluierung}

und Sportlerinnen gefordert. ${ }^{78}$ Es wird darauf hingewiesen, dass die Aussicht auf Strafmilderung oder Straffreiheit Athleten - die oftmals aus Scham, Angst oder falsch verstandener Loyalität schweigen - dazu motivieren könne, gegenüber den Ermittlungsbehörden weitere Angaben zu ihrem Umfeld machen. Auch im internationalen Vergleich habe sich gezeigt, dass die Kooperation mit den überführten Sportlern und Sportlerinnen eine wichtige Voraussetzung für die Aufdeckung von Dopingstrukturen sei.

„Die zuständigen Ermittler der Anti-Doping-Agenturen stellt dieser mangelnde Kooperationswille vor große Probleme. Sie sind oftmals weder in der Lage, alle Mitglieder des Doping-Netzwerks zu identifizieren, noch genügend rechtswirksame Beweise gegen Verdächtigte zu sammeln. (...) In der Vergangenheit haben sich solche Mechanismen als sehr hilfreich erwiesen. Kronzeugenregelungen halfen beispielsweise den amerikanischen Behörden dabei, den weitverbreiteten Dopingmissbrauch im ehemaligen Radsportteam US Postal aufzudecken." "79

Auch in der Literatur wird die Einführung einer Kronzeugenregelung für sämtliche in $\$ 4$ vorgesehene Straftatbestände gefordert. Argumentiert wird mit der Parallele zum Betäubungsmittelrecht, dessen spezielle Kronzeugenregelung in $\$ 31$ BtMG ihrer Ratio nach vergleichbar sei und sich in der Praxis bewährt habe. ${ }^{80}$ Flankiert werden sollte die strafrechtliche Kronzeugenregelung mit der Zusage einer Aufhebung sportrechtlicher Sperren bzw. einer Beendigung des Doping-Disziplinarverfahrens durch die zuständige Anti-Doping-Organisation. ${ }^{81}$

In den Experteninterviews ließen sich zwei Positionen zur Kronzeugenregelung unterscheiden. Einige der Experten bezweifelten die Notwendigkeit einer speziellen Kronzeugenregelung.

"[Ob eine Kronzeugenregelung sinnvoll wäre,] kann ich jetzt so nicht beantworten, weil wir uns sehr, sehr selten im Leistungssport bewegen und mir ist

78 Athleten Deutschland e.V., Stellungnahme in der Anhörung des Sportausschusses am 23.8.2019, S. 4; DAV, Stellungnahme Nr. 38/2019, S. 4; DOSB, Öffentliche Anhörung des Sportausschusses am 23. Oktober 2019, S. 2; NADA, Öffentliche Anhörung des Sportausschusses des Deutschen Bundestages, Änderungs- und Ergänzungsbedarfe im Anti-Doping-Gesetz (AntiDopG), S. 2 f.

79 Stellungnahme Athleten Deutschland e.V., S. 4.

80 Hauptmann/Klarmann, SpuRt 2019, 197.

81 Cherkeh, SpuRt 2019, 167. 
jetzt, ehrlich gesagt, keine Anwendung einer Kronzeugenregelung bei unseren Ermittlungsverfahren bekannt." (Zoll 3)

Interviewer: "Welche Rolle spielt die für $\$ 2$ anwendbare Kronzeugenregelung?

R 1: $\quad$ "Das hat bei uns bisher keine Rolle gespielt."

Interviewer: „Wäre eine Erweiterung der Kronzeugenregelung auf das Selbstdoping sinnvoll?"

R 1 „Nachdem sie bisher keine Rolle gespielt hat: nein."

„[Die Kronzeugenregelung] hat bei uns bis jetzt soweit für mich ersichtlich noch keine Rolle gespielt. Also in meiner Tätigkeit bislang noch nicht. Lässt sich für mich nicht beurteilen. "(StA 5)

„Interviewer: „Welche Rolle spielt die für $\$ 2$ anwendbare Kronzeugenregelung?

StA 7: $\quad$ "Die spielt bei mir keine Rolle.

Interviewer: „Wäre eine Erweiterung der Kronzeugenregelung auf das Selbstdoping sinnvoll?"

StA 7: $\quad$ "Nein, weil sie ja keine Rolle spielt für mich.“

Die Befragten kritisierten allerdings nicht die grundsätzliche Idee einer Kronzeugenregelung, sondern sahen mit Blick auf die eigene Tätigkeit keinen praktischen Anlass für eine Reform. In Anbetracht der derzeit noch geringen Verfahrenszahlen ist dieser Befund jedoch nicht überzubewerten: Da die Staatsanwaltschaften - und erst recht die Gerichte - nur äußerst selten mit einschlägigen Fällen von Selbstdoping konfrontiert waren, liegt auf der Hand, dass bislang kaum Bedarf nach einer Kronzeugenregelung bestand.

Gleichwohl sprachen sich in den Interviews neben den Vertretern von NADA und Athletenverbänden auch Staatsanwälte für eine spezielle Kronzeugenregelung im AntiDopG aus.

„Sie wäre sicherlich wünschenswert. Sie ist einfach für den Beschuldigten durchaus ein Signal, wenn man ibn entsprechend belehren kann, dass er 
durchaus vielleicht motiviert ist, ja weitergehende Angaben zu machen." $(\text { StA } 1)^{82}$

"In meinen Augen ist das AntiDopG ein gutes Gesetz. Man kann mit dem AntiDopG wirklich arbeiten, aber das ist eine echte Lücke. Da kann man wirklich nachbessern. (...) Ich finde, das ist eine ganz zentrale Geschichte. Also wenn man da was machen will, wenn man Dopingverfolgung optimieren will, dann muss man da diese Kronzeugenregelung reinmachen." (StA 3)

bb) Verbesserter Schutz von Hinweisgebern

In den Stellungnahmen der NADA und der Athletenverbände wird darüber hinaus ein verbesserter Schutz von „Whistleblowern“ gefordert. ${ }^{83}$ Dabei geht es um Personen, die Informationen über Dopingverstöße preisgeben möchten, ohne selbst mit einem strafrechtlichen Vorwurf konfrontiert zu sein. Die NADA weist darauf hin, dass „alle Enthüllungen der größten, aktuellen Dopingskandale [...] auf den Aussagen von Hinweisgebern/-innen [basieren]". ${ }^{84}$ Auch in den Interviews wurde von den Vertretern dieser Stellen die Bedeutung eines besseren Hinweisgeberschutzes betont.

Hinweisgeber riskieren durch die Aufdeckung eines Dopingverstoßes schwerwiegende berufliche, finanzielle und private Folgen, da noch immer die Gefahr besteht, im sportlichen Umfeld als „Nestbeschmutzer“ zu gelten. ${ }^{85}$

„Für uns ist aber wichtig, dass auch Personen, die etwas sehen, hinreichend geschützt sind. Auch in dem Skandal von Russland hat man ja gesehen, dass

82 Allerdings fügt der Experte an, dass es sich hierbei um eine allgemeine Einschätzung handelt, die nicht auf Erfahrungswerten beruht: „Wir haben aber, was die bestehenden Möglichkeiten anbelangt, bisher sehr sehr selten festgestellt, dass jetzt ein Beschuldigter allein aufgrund einer entsprechenden Belehrung mehr Angaben macht, aber gleichwohl würden wir es als sinnvolles Signal erachten für den Beschuldigten."

83 Athleten Deutschland e.V., Stellungnahme in der Anhörung des Sportausschusses am 23.8.2019, S. 4; NADA, Öffentliche Anhörung des Sportausschusses des Deutschen Bundestages, Änderungs- und Ergänzungsbedarfe im Anti-Doping-Gesetz (AntiDopG), S. 3.

84 NADA, Öffentliche Anhörung des Sportausschusses des Deutschen Bundestages, Änderungs- und Ergänzungsbedarfe im Anti-Doping-Gesetz (AntiDopG), S. 2.

85 Hauptmann/Klarmann, SpuRt 2019, 191. 
es Länder gibt, wo Sportler sehr, sehr großen Gefahren ausgesetzt sind. Selbst in Deutschland ist es so, dass teilweise kriminelle Strukturen dahinterstehen. Die Personen, die dann etwas sagen, müssen auf jeden Fall wissen, dass sie geschützt sind. Viele sagen auch aus Angst nichts. Das ist, glaube ich, ein großer Punkt, der verbessert werden muss. " (A 1)

In den Interviews wurde insbesondere von Seiten der Athletenvertreter darauf hingewiesen, dass bestehende Hinweisgebersysteme - etwa bei der NADA oder der World Anti-Doping Agency (WADA) - bislang kaum bekannt seien.

„Das Problem ist, dass es viel zu unbekannt ist. Die meisten Athleten wissen nicht einmal, dass es das gibt. (...) Wir hatten eine kleine interne Umfrage bei den Athletenvertretern, das sind die Personen, die sich schon mehr sportpolitisch interessieren und noch viel mehr E-Mails lesen und sich auf Seiten informieren, und von diesen 35 Personen kannten nur zwei Personen - oder drei Personen inkl. mir - das Whistleblowersystem. Man kann sich dann vorstellen, wie das bei Sportlern ist." (A 1)

Ein gutes Whistleblowersystem sollte nach Ansicht der Befragten vollständig anonym sein und gleichzeitig - etwa im Wege einer „Briefkastenfunktion“ - Rückfragen an den Hinweisgeber ermöglichen. Konkrete Vorschläge an den Gesetzgeber wurden von den Experten allerdings nicht unterbreitet. Die Einrichtung effektiver Hinweisgebersysteme wurde vorrangig als Aufgabe der Verbände und Anti-Doping-Agenturen gesehen:

„Ob das Anti-Doping-Gesetz [für Regelungen zum Hinweisgeberschutz] der richtige Ort ist, weiß ich nicht. Wir selber haben die Initiative auf den Weg gebracht, zu sagen, Whistleblower im Sport müssen grundsätzlich geschützt werden. Das ist eher eine Aufgabe aus dem Sportcomment. (...) Also, da fehlt mir so ein bisschen der konkrete Ansatz, wie sich ein Gesetzgeber an die Spitze stellen kann. Nichtsdestotrotz würde ich mir natürlich eine gewisse Initiative wünschen, wenn die möglich ist. " $(N)$

\section{Die Verbotsnormen des $\$ 2$}

\2 ist eine von zwei zentralen Verbotsnormen des AntiDopG. $\mathbb{2}$ Abs. 1 enthält Tatbestände, die die „Abgabeseite“86 des Dopings adressieren und die insbesondere die Herstellung, das Handeltreiben und das Inverkehr-

86 Treffend Weber BtMG/ders., 5. Aufl. 2017, AntiDopG $₫ 2$ Rn. 2. 


\section{B. Ergebnisse der Evaluierung}

bringen von Dopingmitteln verbieten. $\$ 2$ Abs. 2 erfasst die unerlaubte Anwendung von Dopingmitteln und -methoden bei anderen, während $\mathbb{} 2$ Abs. 3 den Erwerb und Besitz von Dopingmitteln in nicht geringer Menge zum Zweck des Dopings beim Menschen sowie deren Verbringung in bzw. durch den Geltungsbereich des AntiDopG verbietet. \4 Abs. 1 Nr. 1-3 stellt Verstöße gegen diese Verbotsnormen unter Strafe.

Diese Regelungen stehen nach Auskunft aller befragten Personen beim Zoll, den Staatsanwaltschaften und den Gerichten im Zentrum ihrer praktischen Arbeit mit dem AntiDopG. Repräsentativ ist folgende Aussage eines Staatsanwaltes:

„Die Verstöße gegen $\mathbb{S} 2$, also den Besitz von Dopingmitteln in nicht geringer Menge, die stellen ja den Schwerpunkt unserer Arbeit, unserer Ermittlungsverfahren dar. Also ohne diese Regelung hätten wir wenig Kontakt mit dem AntiDopG selbst (...). “(StA 6)

Bestätigt wird diese Aussage von einem anderen Staatsanwalt, der zugleich aufzeigt, welche Personengruppe den Großteil der Beschuldigten ausmacht:

„90\% der Fälle sind natürlich, dass am Frankfurter Flughafen viele Pakete vom Zoll, die Testosteronampullen o.̈. enthalten, festgestellt werden, die ein Freizeitsportler über ein Internetportal erworben hat (...) und das meistens aus Thailand, China oder den USA nach Frankfurt verfrachtet und das dann durch den Zoll abgefangen wird. Von diesen $90 \%$ würde ich wiederum sagen: $80 \%$ sind Freizeitsportler, die ins Fitnessstudio gehen, Muskelaufbau betreiben." (StA 2)

\section{Phänomen}

a) Erkenntnisse zu Tätern und Tathandlungen

Die Aussagen der interviewten Ermittler des Zolls, der Staatsanwaltschaften und von Richtern zu den Beschuldigten fielen einheitlich aus. Danach betreffen die Verfahren fast ausnahmslos „(d)ie Fitness-Szene im Allgemeinen, also sagen wir mal Breitensport, klassische Fitnessstudio-Besucher, also Kraftsport und Bodybuilding. “ (Zoll 3). Der „Bereich Muskelaufbau, Bodybuilding“" sei „wirklich der größte Teil unserer Verfahren. " (StA 1)

Diese Angaben lassen sich in zweifacher Hinsicht auffächern. Zum einen richten sich die Ermittlungsverfahren nicht nur gegen Kraftsportler, die „das wirklich auch wettkampfmäßig tu[en], sondern auch (gegen) Leute, die 
ibren Körper einfach in Form bringen wollen. Das ist so das Gros an Fällen, die wir haben." (StA 5)

„Der überwiegende Anteil der Verfahren, die wir in der Schwerpunktstaatsanwaltschaft bearbeiten, sind Verfahren, die Bodybuilder betreffen oder Kraftsport. Bodybuilder, die das teilweise auf Wettkampfniveau betreiben, aber der wesentliche Teil bei den Bodybuildern sind einfach Personen, die ins Fitnessstudio gehen und dort dem Sport nachgehen. Das ist so der zablenmäßig größte Teil." (StA 4)

„Also Hauptempfänger sind ganz klar der Bodybuildersport. Angefangen von Leuten, die es aktiv machen, die also Wettkämpfe betreiben, aber auch ein großer Teil Freizeitsport in den Fitnessstudios, die Kraftzuwächse erwarten, um dem aktuellen Lifestyle zu entsprechen. Und das ist zu über 90\% unser Empfängerkreis. " (Zoll 1)

Zum anderen zeigen die Interviews, dass Selbstnutzer den Großteil der Beschuldigten ausmachen, also Personen, die Dopingmittel zum Eigengebrauch erwerben, besitzen oder in den Geltungsbereich des Gesetzes verbringen. Diejenigen, die ausschließlich oder primär Dopingmittel an Dritte abgeben, diese herstellen oder mit ihnen Handel treiben, sind hingegen deutlich unterrepräsentiert. Im Zentrum der Strafverfolger stehen also „ganz klar Selbstnutzer" (StA 7); die Beschuldigten sind überwiegend „nur Verbraucher" (R 1).

Seltener kommt es vor, dass sich Ermittlungsverfahren gegen Hersteller oder Händler richten. Ein Befragter berichtete von einem besonders gelagerten Fall der Herstellung von Dopingmitteln und gewerbsmäßigem Handeltreiben:

„Ein konkreter Fall, der tatsächlich etwas größer ist, ist ein Ehepaar, das über einen noch nicht mal so ewig großen Zeitraum Mittel aus China bestellt hat. Rohstoffe aus China. Und hat dann - obne eine große Vorkenntnis in Hinsicht auf chemische Prozesse - Mischungen erstellt, maßgeblich Testosteronprodukte, und diese dann in einem erheblichen Maße über Ebay verkauft." (StA 5)

Häufiger sind indes Fälle, in denen ein Beschuldigter Dopingmittel zum Eigengebrauch erworben hat oder besitzt und zugleich damit (im geringen Umfang) Handel treibt. Ein Richter sagte dazu:

„Entweder war es der Bodybuilding-Studiobetreiber, der aber selbst das Zeug auch genommen hat und an seine Kunden weitergegeben hat oder der Bodybuilder selber. Da waren dann glaube ich auch ein oder zwei dabei, die das 
dann en gros bestellt haben und das dann weiterveräußert haben, so dass die dann in den Verbrechenstatbestand reingerutscht sind. Aber im Grunde genommen sind es die typischen Selbstschädiger. " $(R 2)$

„Handel treiben, das kommt immer mal, wie auch die Betäubungsmittelabbängigen, wenn die was einkaufen und Teile davon abgeben, um quasi sich das Geld für die nächste Charge zu besorgen." ( $R 2)$

Dessen ungeachtet werden strukturelle Parallelen zu anderen Formen der organisierten Kriminalität von Ermittlern des Zolls und auch einzelnen Staatsanwälten betont, insbesondere ein „arbeitsteiliges, sehr konspiratives Vorgehen." (Zoll 3). Es gebe „auch des Öfteren mal Berührungspunkte zur Rockerszene." (Zoll 3) Dabei werden vor allem die hohen Gewinnmargen als Grund dafür genannt, weshalb der organisierte Handel mit Dopingmitteln für Straftäter interessant sei:

„Ja, ganz klar. Die Gewinnmarge im Bereich mit Handel von Dopingmitteln ist höher als die im Handel mit Betäubungsmitteln. Bis vor ein paar Jahren war die Strafandrohung auch wesentlich geringer, so dass man davon ausgehen konnte, dass es sicherer war, mit Dopingmitteln Handel zu treiben. Da konnte man mehr Gewinne generieren als mit dem Rauschgifthandel und die Bestrafung war ja auch gar nicht vergleichbar mit der Bestrafung des Handels mit Betäubungsmitteln." (Zoll 1)

Bestehen entsprechende Anhaltspunkte für organisierte Tätergruppen, versuchen die Ermittler diese Netzwerke aufzudecken:

„Natürlich versuchen wir die Hintermänner zu bekommen. Wir versuchen insbesondere hier zu ermitteln. Ab einer gewissen Menge an Einfuhrvolumen oder auch an den Produkten kann man abschätzen, ob der Empfänger hier ein Konsument ist oder jemand, der es weiterverarbeitet. Unsere Bestrebung ist, die Hintermänner zu bekommen, insbesondere die hier auch den Reibach damit machen, das heißt, die Dopingmittelwirkstoffe umarbeiten und dann letztendlich damit Handel treiben." (Zoll 1)

„Wir haben am Frankfurter Flughafen auch schon große Frachtsendungen angehalten, die dann von einem Betreibernetzwerk sozusagen erlangt werden sollten. Die gibt es, aber in wenigen Fallzablen. Die kommen mal vor, man kann vielleicht sagen, alle eineinhalb Jahre machen wir Strukturermittlungen zu so großen Fällen." (StA 7) 
b) Erkenntnisse zu Dopingmitteln

Die Befragungen zu den verfahrensgegenständlichen Dopingmitteln ergaben drei wesentliche Erkenntnisse. Zum einen dominieren klassische Präparate zum Muskelaufbau:

"Letztendlich fangen wir an mit dem Klassischen, dem Testosteron, Nandrolon, aber auch die Sachen, die man braucht, um die Nebenwirkungen abzufedern von Tamoxifen über Clomifen. Also alles was man sich vorstellen kann. Unser Haupttäterkreis befasst sich mit Freizeitsport, sprich im Kraftsport, Bodybuilding - und alles, was Muskelzuwachs bewirkt, wird auch letztendlich eingeführt. Zum Teil als Fertigprodukt, zum Teil als Rohware als Wirkstoff, der dann weiter verarbeitet wird zu diesen entsprechenden Dopingmitteln."(Zoll 1)

Zum anderen lassen sich nach Auskunft der Ermittler auch Trends beobachten, in deren Folge neue Dopingmittel rasch große Beliebtheit erlangen:

„Es gibt jede Menge neue Fancy-Stoffe. (...) Seit einiger Zeit jede Menge von den neuen Sarms [selektive Androgenrezeptor-Modulatoren] zum Beispiel." (Zoll 3)

Zum Dritten betreffen Ermittlungen zunehmend Lifestyle-Präparate, die grundsätzlich zum Zweck des Dopings im Sport eingesetzt werden können, häufig aber anderen Zwecken dienen:

„Seit neuestem dieses DHEA. Und Epiandrosteron (...) kommt auch sehr oft vor. Wobei das dann weniger zum Muskelaufbau verwendet wird, sondern um das normale Altern und Sexualverlangen wieder in den Griff zu bekommen in einem gewissen Alter." (StA 2)

Nach Aussage eines Zollermittlers verursachen Verfahren, die solche Lifestyle-Präparate betreffen, erheblichen Aufwand. Die konkrete Feststellung, ob sie tatsächlich Dopingzwecken dienen sollen, sei arbeitsintensiv und mitunter kaum ermittelbar.

„Wir werden ganz stark damit frequentiert. (...) Wir haben jedoch keinerlei Erkenntnisse, dass dieses Präparat, obwobl es doch gelistet ist und wir dann (...) das Ermittlungsverfahren zu betreiben haben, (...) überhaupt zu sol- 
chen Zwecken herangezogen werden sollte. (...) Das bindet ganz schön Personal." (Zoll 2) ${ }^{87}$

c) Zusammenfassung

Im Zentrum der Strafverfolgung von Taten nach dem AntiDopG stehen Verstöße gegen $\mathbb{2}$. Innerhalb dieser Gruppe von Verstößen richten sich die Ermittlungen zumeist gegen Selbstnutzer von Dopingmitteln, die Bodybuilding und Fitness außerhalb von Wettkämpfen als Breitensport betreiben. Dementsprechend handelt es sich bei der großen Mehrheit der verfahrensgegenständlichen Dopingmittel um Präparate, die zum Muskelaufbau verwendet werden. Hinzu treten in zunehmendem Ausmaß neue „Fancy“-Präparate bzw. Lifestyle-Produkte, die oftmals nicht zu Dopingzwecken verwendet werden.

2. Feststellungen zu materiell-rechtlichen Aspekten von $₫ 2$

a) Die Tatbestände und ihre Voraussetzungen

aa) Legislativer Kontext

Gemäß $₫ 2$ Abs. 1 ist es verboten, ein Dopingmittel, das ein in der Anlage I des Internationalen Übereinkommens vom 19. Oktober 2005 gegen Doping im Sport in der vom Bundesministerium des Innern jeweils im Bundesgesetzblatt Teil II bekannt gemachten Fassung aufgeführter Stoff ist oder einen solchen enthält, zum Zwecke des Dopings beim Menschen im Sport

- herzustellen,

- mit ihm Handel zu treiben,

- es, ohne mit ihm Handel zu treiben, zu veräußern, abzugeben oder sonst in den Verkehr zu bringen oder zu verschreiben.

\$2 Abs. 2 verbietet es, ein solches Dopingmittel zum Zwecke des Dopings im Sport bei einer anderen Person anzuwenden. Schließlich ist es nach $\mathbb{} 2$ Abs. 3 verboten, ein solches Dopingmittel, das ein in der Anlage zum AntiDopG aufgeführter Stoff ist oder einen solchen enthält, in nicht geringer

87 Näher zu den Schwierigkeiten bei der Feststellung des Verwendungszwecks unten 2.a.ee. 
Menge zum Zwecke des Dopings beim Menschen im Sport zu erwerben, zu besitzen oder in oder durch den Geltungsbereich dieses Gesetzes zu verbringen.

$\$ 4$ Abs. 1 Nr. 1-3 stellen Handlungen, die diesen Verboten zuwiderlaufen, unter Strafe, wobei $\$ 4$ Abs. 5 auch fahrlässiges Handeltreiben unter Strafandrohung stellt.

Diese Regelungen dienen, so der Gesetzgeber, „in erster Linie dem Gesundheitsschutz“. ${ }^{88}$ Denn die Anwendung von Dopingmitteln und Dopingmethoden zum Zwecke des Dopings im Sport beruhe nicht auf einer medizinischen Indikation und führe daher zu einem aus medizinischer Sicht nicht angezeigten Eingriff in den Körper, der erhebliche Gefahren für die Gesundheit der betroffenen Sportlerinnen und Sportler mit sich bringe. Zahlreiche Todesfälle in der Vergangenheit und schwere Spätfolgen systematischen Dopings belegten die Schädlichkeit des Dopings. Auch minderjährige Sportlerinnen und Sportler gelangten an Dopingmittel und gebrauchten diese trotz der besonderen Gesundheitsgefahren, die die Anwendung dieser Mittel gerade bei jungen Menschen birgt. ${ }^{89}$ Betroffen seien nicht nur die Sportlerinnen und Sportler, sondern auch die Allgemeinheit, die die Kosten der Behandlung trage. Zudem verweist die Gesetzesbegründung auf den illegalen Handel mit Dopingmitteln, der eine alarmierende Dimension erreicht habe und sich organisierter Vertriebswege und Händlerstrukturen bediene, die mit denen im organisierten Rauschgifthandel vergleichbar sind.

$\$ 2$ übernimmt die zuvor im AMG enthaltenen Verbotsnormen und stellt diese - in veränderter Form - in den Kontext des Dopings im Sport. Die Ausgliederung der Verbotsnormen aus dem AMG und ihre Einbindung in das AntiDopG wird positiv bewertet:

"[Unsere Arbeit] hat sich in dem Sinne erleichtert, dass die Rechtsanwendung einfacher geworden ist und wir nicht mehr im Arzneimittelgesetz rumstöbern müssen." (Zoll 3)

88 BT-Drs. 18/4898, 23; Erbs/Kohlhaas/Wußler, 228. EL Januar 2020, AntiDopG $₫ 2$ Rn. 1.

89 BT-Drs. 18/4898, S. 53.; Eising, Die Strafbarkeit des Eigendopings, 2018, S. 86; Heger, medstra, 2017, 205, 212; vgl. insofern auch die Parallelvorschrift des $\mathbb{\$} 29 \mathrm{a}$ Abs. 1 Nr. 1 BtMG zur unerlaubten Abgabe von Betäubungsmitteln an Minderjährige: Körner/Patzak/Volkmer/Patzak BtMG, 9. Aufl. 2019, \29a Rn. 5. 
Auch die größere Normklarheit wird betont:

„Die (Verbotsnormen) sind sehr schmal und sehr klar verständlich (...).“ (Zoll 1)

Als ein Vorteil der Neuregelung wird gesehen, dass die Anwendbarkeit der Verbotsnormen nun nicht mehr an die Arzneimitteleigenschaft gekoppelt ist, sondern an den weitergefassten Begriff des „Dopingmittels“. Zur früheren Rechtslage nach dem AMG führte ein Ermittler aus:

„Um eine Strafbarkeit zu erreichen, mussten wir mit der [Tatsache] ,umgehen', dass es sich bei den Dopingmitteln oft um gefälschte Arzneimittel handelt. [...] Um eine Verurteilung zu erreichen, mussten wir uns damals Hilfsbrücken bedienen." (Zoll 1)

Das Gesetz definiert den Begriff des „Dopingmittels“ nicht. Die Gesetzesbegründung führt aber aus, dass sowohl Arzneimittel als auch sonstige Wirkstoffe erfasst sein sollen. ${ }^{90}$ Entscheidend für die Anwendbarkeit der $\$ 2$ Abs. 2 und 3 ist, dass Dopingmittel solche Stoffe enthalten, die in der Anlage 1 des Internationalen Übereinkommens gegen Doping im Sport vom 19. Oktober 2005, in der vom Bundesministerium des Inneren im Bundesgesetzblatt Teil II jeweils bekannt gemachten Fassung aufgeführt werden. Anders als nach $\$ 6$ a Abs. 2 S. 1 AMG a.F. werden also nicht sämtliche Änderungen der Anlage I des Übereinkommens in den Verbotstatbestand einbezogen. Vielmehr erlangen solche Änderungen auf internationaler Ebene innerstaatliche Wirkung für das AntiDopG erst durch eine Entscheidung des nationalen Verordnungsgebers. ${ }^{91}$

Der BGH hat in einem Beschluss vom 14.2.2019 darin ein ausreichendes „Maß an nationaler Gestaltungshoheit" gesehen ${ }^{92}$ und aus diesem Grund verfassungsrechtliche Bedenken gegen die Inbezugnahme der Anlage I des Internationalen Übereinkommens gegen Doping zurückgewiesen. ${ }^{93}$ Praktiker bewerten diesen Ansatz als positiv, weil man ,mit Hilfe der Stoffliste und der Verbotsliste" den Bereich des Strafbaren leicht bestimmen könne (Zoll 1).

Kritiker monieren indes, dass - anders als etwa im BtMG - keine Rechtsverordnung für die Bestimmung der inkriminierten Arzneimittel und

90 BT-Dr 18/4898 S. 23; krit. Weber BtMG/ders., AntiDopG $\$ 2$ Rn. 6.

91 BT-Drs. 18/4898, S. 24.

92 BGH v. 14.2.2019, 4 StR 283/18, StV 2020, 315, 317. Siehe ferner Finken, PharmR 2016, 445, 446: „Die Regelung begegnet deshalb keinen verfassungsrechtlichen Bedenken."

93 BGH v. 14.2.2019, 4 StR 283/19, StV 2020, 315, 317. 
Wirkstoffe erforderlich sei, sondern eine bloße Bekanntmachung der Übernahme der Geltung von Änderungen der Anlage 1d des Dopingübereinkommens: Eine Rechtsverordnung habe - auch im Hinblick auf das Erfordernis des Gesetzlichkeitsprinzips - einen anderen Status als eine Bekanntmachung und zudem andersartige Verfahrensvoraussetzungen. ${ }^{94} \mathrm{Ge}-$ fordert wird daher, dass im AntiDopG eine neue Verordnungsermächtigung zur Festlegung inkriminierter Stoffe geschaffen wird, um fortan im Wege der Rechtsverordnung - anstatt einer Bekanntmachung - die Tatobjekte des AntiDopG genauer zu bestimmen:

„Auch bei der Bekämpfung des Dopings im Sport wäre es jedenfalls ein Gewinn an Rechtsstaatlichkeit, wenn eine solche Verordnungsermächtigung geschaffen oder, solange dies nicht geschehen ist, statt einer Bekanntmachung von der Verordnungsermächtigung des $\$ 6$ Abs. 2 Gebrauch gemacht wür$d e .{ }^{\text {"9 } 95}$

Der Gesetzgeber verweist hingegen darauf, dass gerade bei der Dopingbekämpfung besondere Flexibilität nötig sei, um - auch „unterjährig“ und innerhalb weniger Monate - schnell und sachgerecht auf neue Entwicklungen reagieren zu können. In der Vergangenheit habe sich gezeigt, dass „eine hohe Gefahr besteht, hinter der raschen Entwicklung neuer Dopingmittel und Dopingmethoden zurückzubleiben, was den effektiven Kampf gegen Doping beeinträchtigt. ${ }^{\text {996 }}$

bb) Herstellung, Handeltreiben, Inverkehrbringen, Verschreiben $(\mathbb{2}$ Abs. 1)

Gemäß 6a AMG a.F. war es verboten, Arzneimittel zu Dopingzwecken im Sport in den Verkehr zu bringen, zu verschreiben oder bei anderen anzuwenden, sofern ein Doping bei Menschen erfolgt oder erfolgen soll. $\$ 2$ Abs. 1 hat diese Verbote in veränderter Form übernommen und um neue Tatbegehungsweisen erweitert. ${ }^{97}$ Nach Auffassung des Gesetzgebers hatten sich die Regelungen des AMG als zu eng erwiesen; auch das Herstellen,

94 Weber BtMG/ders., AntiDopG, 5. Aufl. 2017, 2 Rn. 14 f. Ferner Graf/Jäger/ Wittig/Eschelbach, Wirtschafts- und Steuerstrafrecht, 2. Aufl. 2017, $\$ 4$ AntiDopG Rn. 1, 11.

95 Weber BtMG/ders., 5. Aufl. 2017, AntiDopG $\$ 2$ Rn. 15.

96 BT-Drs. 18/4898, S. 25.

97 Weber BtMG/ders., 5. Aufl. 2017, AntiDopG $₫ 2$ Rn. 6. 


\section{B. Ergebnisse der Evaluierung}

das Handeltreiben, das Veräußern und die Abgabe von Dopingmitteln seien im Hinblick auf die verursachten Gesundheitsgefahren strafwürdig. ${ }^{98}$

Mit dem Verbot des Herstellens soll das Entstehen illegaler Märkte bekämpft werden. ${ }^{99}$ Das Handeltreiben wurde in den Katalog der Tatbegehungsweisen aufgenommen, da dieser eigennützigen, auf Umsatz gerichteten Tätigkeit nach Auffassung des Gesetzgebers eine große praktische Bedeutung zukomme. ${ }^{100}$ Mit der Aufnahme der Tatbestandsvariante der Veräußerung sollen die Fallgruppen abgedeckt werden, in denen die Täterin oder der Täter zwar entgeltlich, aber uneigennützig Dopingmittel abgibt. ${ }^{101}$ Um Regelungslücken bei nicht konkret nachweisbaren Entäußerungshandlungen zu schließen, wurde der Auffangtatbestand des sonstigen Inverkehrbringens in die Verbotsnorm mit aufgenommen. ${ }^{102}$

Die befragten Experten lobten die Neufassung aus zwei Gründen. Zunächst beseitige sie bisher bestehende Auslegungsunklarheiten bzw. Strafbarkeitslücken. Insbesondere die Aufnahme des Handeltreibens wird als positiv erachtet, da manche Fälle nicht von der Tathandlung des Inverkehrbringens erfasst werden:

„Was gut ist, dass das Handeltreiben aufgenommen worden ist, weil das in den Verkehr bringen ja immer so eine Art Vorratshaltung erfordert hat und damit Vorfälle im Vorfeld, wie z. B. eine Bestellung oder ein abgefangenes Paket immer schwierig war, rechtlich zu greifen. "(StA 3)

Die neue Rechtslage brächte eine Erleichterung ${ }^{103}$ mit sich, da sie auch „das verbale Handel treiben, also bis zur ernsthaften verbindlichen Einigung im Vorfeld als vollendetes Handeltreiben" (StA 3) erfasse, und damit alle Fälle „vom Packen eines Pakets, das aber nicht wegeschickt wird, bis hin zu einem Paket, das weggeschickt und von der Post abgefangen wird" (StA 3).

98 BT-Drs. 18/4898, S. 23; Lehner/Nolte/Putzke/Striegel, Anti-Doping-Gesetz, $\mathbb{} 2$ Rn. 12.

99 BT-Drs. 18/4898, S. 23; Graf/Jäger/Wittig/Eschelbach, Wirtschafts- und Steuerstrafrecht, 2. Aufl. 2017, AntiDopG $\ 4$ Rn. 5.

100 Der Begriff des Handeltreibens lehnt sich an die Begrifflichkeit des Betäubungsmittelrechts an, BT-Drs. 18/4898, S. 24; Weber BtMG/ders., 5. Aufl. 2017, AntiDopG $₫ 2$ Rn. 35.

101 BT-Drs. 18/4898, S. 24; MüKo-StGB/Freund, 3. Aufl. 2018, AntiDopG, $\mathbb{\$} \$ 1-4$ Rn. 50.

102 BT-Drs. 18/4898, S. 24; Weber BtMG/ders., 5. Aufl. 2017, AntiDopG $\ 2$ Rn. 55.

103 „Das war, finde ich, eine große Erleichterung.“ (StA 3) 
Ferner wird gelobt, dass sich die Tatbestandsformulierungen an etablierte Formulierungen des BtMG anlehnen:

„Die neue Rechtslage ist zu begrüßen, auch weil sie eine Nähe zum Betäubungsmittelstrafrecht herbeifübrt."

Dies sei zum einen wichtig für Verteidiger und andere Rechtsanwender, die „den ersten Fall wegen Verstoßes gegen das Anti-Doping-Gesetz" hätten und sich eher im BtMG „zu Hause füblen“. Zum anderen sei es von Vorteil, wenn man auf Begrifflichkeiten zurückgreifen könne, „die bereits durch die Rechtsprechung zum BtMG geprägt" sind. (StA 1)

Die Vorteile aus Sicht der Strafverfolgungsbehörden fasst ein Staatsanwalt wie folgt zusammen:

„Was natürlich für den $\$ 2$ spricht, ist, dass er klar formuliert ist. Es ist ja im Endeffekt auch ganz nah am BtMG angelehnt." (StA 5)

cc) Anwendung von Dopingmitteln und -methoden ( $\$ 2$ Abs. 2)

Das Verbot der Anwendung von Dopingmitteln (Nr.1) entspricht dem bisher geltenden Verbot in $\$$ 6a Absatz 1 AMG. Erweitert wird die Regelung um das Verbot der Anwendung von Dopingmethoden bei anderen (Nr. 2). Die Dopingmethoden ergeben sich - wie auch die Dopingmittel im Einzelnen aus der Anlage I zum Internationalen Übereinkommen gegen Doping im Sport in der vom Bundesministerium des Innern bekanntgemachten Fassung.

Mit der ausdrücklichen Erfassung auch von Dopingmethoden hat der Gesetzgeber beabsichtigt, Schutzlücken zu schließen. ${ }^{104}$ Dies begrüßt ein Staatsanwalt unter Verweis auf ein Fallbeispiel:

„Also [die Einbeziehung von Dopingmethoden] schließt Lücken und sie ist insoweit sehr zu begrüßen. (...) [Wir] hatten vor einiger Zeit in einem Verfahrenskomplex tatsächlich festgestellt, dass da verbotene Methoden angewandt wurden. Das Verfahren hätte es so nicht gegeben, weil es da tatsächlich nur um Methoden ging. Wenn man eben jetzt nur verbotene Stoffe gehabt hätte, hätte das Verfahren sofort zur Einstellung geführt. So hat das eben zur Beantragung von Strafbefehlen geführt."(StA 1)

104 BT-Drs. 18/4898, S. 25; Körner/Patzak/Volkmer/Volkmer, BtMG, 9. Aufl. 2019, AntiDopG vor $\mathbb{1} 1$ Rn. 21; Weber BtMG/ders., 5. Aufl. 2017, AntiDopG $\$ 1$ Rn. 2. 


\section{B. Ergebnisse der Evaluierung}

Ein anderer Staatsanwalt pflichtet dem bei und verweist auf Rechtsunsicherheiten zur früheren Rechtslage nach dem AMG und führt ebenfalls ein konkretes Ermittlungsverfahren an:

„Also was sicher wichtig war, war die Aufnahme von Dopingmethoden ins Gesetz. Zum Zeitpunkt des AMGs war die Erfassung der Methoden ja immer streitig; ob also eine Methode auch Arzneimittel sein kann. Insbesondere das Blutdoping, das ja auch bei der Operation ,Aderlass" eine Rolle spielt." (StA 3)

Kritisch äußerte sich lediglich ein Richter, der indes noch keine Erfahrung mit diesem Tatbestand gesammelt hat:

„Ich habe Zweifel an der Notwendigkeit. Nein, wir haben nie über die Anwendung bestimmter Dopingmethoden zu verhandeln gehabt." ( $R$ 1)

dd) Erwerbs-, Besitz- und Durchverbringung nach $₫ 2$ Abs. 3

(1) Allgemeine Bedeutung

Der Absatz übernimmt aus dem bisher geltenden $\$$ 6a Abs. 2a Satz 1 AMG das Verbot von Besitz und Erwerb nicht geringer Mengen bestimmter gesundheitsgefährdender Dopingmittel zu Dopingzwecken. Hinzu tritt das Verbot des Verbringens in oder durch den Geltungsbereich dieses Gesetzes, also in oder durch das Gebiet der Bundesrepublik Deutschland. Damit wird das Verbot des Verbringens von Arzneimitteln in den Geltungsbereich ( $\$ 73$ AMG) um die Variante des Verbringens von Dopingmitteln durch den Geltungsbereich des Gesetzes ergänzt.

(2) Erwerb und Besitz nicht geringer Mengen

In der Praxis scheint insbesondere den Tatvarianten des Erwerbs und Besitzes eine besondere Bedeutung zuzukommen. Darauf deuten die oben unter 1a) zitierten Aussagen hin, wonach Beschuldigte zumeist "ganz klar Selbstnutzer" (StA 7) seien. Zudem antwortet ein Ermittler des Zolls auf die Frage nach einem typischen Beispiel für seine Ermittlungstätigkeit:

„Im Fokus steht hier, glaube ich, $\mathbb{} 4$ Abs. 1 Nr. 3 (i.V.m. $\$ 2$ Abs. 3), nach dem wir agieren." (Zoll 2) 
Der Gesetzgeber hat Erwerb und Besitz nicht geringer Mengen Dopingmittel verboten, da solche Besitzmengen erfahrungsgemäß Vorstufen für einen Handel mit Dopingmitteln darstellten. ${ }^{105}$ Schutzgut der Verbotsnorm ist danach die Gesundheit der Allgemeinheit, die vor der Inverkehrgabe der Mittel zu Dopingzwecken bewahrt werden soll. Zu diesem Zweck sollen bereits Handlungen, die typischerweise nur der Vorbereitung für die Weitergabe dienen, verboten werden. ${ }^{106}$ Damit werden zugleich Beweisprobleme in Fällen gelöst, in denen ein Handeltreiben, Veräußern oder eine Abgabe nicht sicher nachweisbar sind.

Zentrale Bedeutung kommt in diesem Legitimationsmodell dem Merkmal der nicht geringen Menge zu. ${ }^{107}$ Wird die Grenze zur nicht geringen Menge überschritten, vermutet das Gesetz - nach seiner Ratio - unwiderleglich die Absicht der Weitergabe; unterhalb dieser Schwelle kann eine Verwendung zum Eigengebrauch nicht ausgeschlossen und folglich eine Absicht zur Weitergabe nicht unterstellt werden. ${ }^{108}$ Dieser Ansatz gilt in der Literatur grundsätzlich als unbedenklich. ${ }^{109}$ Gelegentlich werden hingegen verfassungsrechtliche Bedenken hinsichtlich der gesetzlichen Bestimmtheit (Art. 103 Abs. 2 GG) geäußert, weil $\$ 6$ Abs. 1 das Bundesministerium für Gesundheit ermächtigt, die nicht geringe Menge per Rechtsverordnung zu bestimmen, ohne dass deutlich würde, an welchem Maßstab der Verordnungsgeber seine Festlegungen vornehmen solle. ${ }^{110}$ Der BGH hält die Vorschrift hingegen für verfassungskonform. Der Gesetzgeber habe die Tathandlung und den Strafrahmen selbst konkret bestimmt: die Festlegung der nicht geringen Menge sei lediglich eine Spezifizierung der strafbegründenden Merkmale. ${ }^{111}$ Auch in den Befragungen wurden keinerlei Bedenken gegen die Zuständigkeit des Verordnungsgebers geäußert.

Die Evaluierung hat indes gezeigt, dass sich die Rechtsanwendung von der oben genannten Legitimationsgrundlage - Verbot des Erwerbs und Be-

105 BT-Drs. 18/4898, S. 25; MüKo-StGB/Freund, 3. Aufl. 2018, AntiDopG $\$ \mathbb{S} 1-4$ Rn. 58; Körner/Patzak/Volkmer/Volkmer, BtMG, 9. Aufl. 2019, AntiDopG, vor $\$ 1$ Rn. 21.

106 BT-Drs. 18/4898, S. 25 f.; BGH StV 2018, 302.

107 Dazu und zum Folgenden Graf/Jäger/Wittig/Eschelbach, Wirtschafts- und Steuerstrafrecht, 2. Aufl. 2017, $\$ 4$ AntiDopG Rn. 17.

108 Weber BtMG/ders., AntiDopG $\ 2$ Rn. 21.

109 Graf/Jäger/Wittig/Eschelbach, Wirtschafts- und Steuerstrafrecht, $\mathbb{4}$ AntiDopG Rn. 17.

110 Graf/Jäger/Wittig/Eschelbach, Wirtschafts- und Steuerstrafrecht, $\mathbb{} 4$ AntiDopG Rn. 1; Körner/Patzak/Volkmer/Volkmer BtMG, 9. Aufl. 2019, AntiDopG $\mathbb{} 4$ Rn. $50 \mathrm{f}$.

111 BGH StV 2020, 315, 317. 
sitzes nicht als Selbstzweck, sondern zur Unterbindung eines Handelns mit Dopingmitteln - deutlich entfernt. Dies aus zwei Gründen. Erstens soll nach Auffassung des BGH $₫ 2$ Abs. 3 auch den Besitz zum Eigendoping erfassen: Einer Strafbarkeit des Angeklagten nach $₫ 4$ Abs. 1 Nr. 3 stünde deshalb nicht entgegen, dass der Angeklagte die Dopingmittel grundsätzlich nur zum Zwecke des Eigendopings besaß und allenfalls zu einer nicht gewinnbringenden Weitergabe bereit war. ${ }^{112}$ Damit lässt sich die Vorschrift auch zur Bestrafung von selbstdopenden Wettkampfsportlern aktualisieren, die nicht die persönlichen Strafanwendungsvoraussetzungen des $\$ 4$ Abs. 7 erfüllen und Dopingmittel ausschließlich zur Selbstapplikation besitzen. Zweitens weisen viele Befragte darauf hin, dass der Schwellenwert für die Annahme einer geringen Menge äußerst niedrig sei. Aus diesem Grund ließ ein Staatsanwalt grundsätzliche Zweifel an der Legitimation der Norm durchscheinen:

„Ich glaube, dass sich der Gesetzgeber da so ein bisschen schwergetan hat, weil das Doping ist ja prinzipiell eine Selbstschädigungssache. (...) Der Gesetzgeber hat halt (...) gesagt, dass der Besitz einer größeren Menge immer die Gefahr birgt, dass es auf den Markt kommt. (...) Man sagt im Prinzip, ob der Besitz von Dopingmitteln strafbar sein muss, wissen wir nicht, aber jedenfalls, wenn da die Gefahr des Handelns oder des Abgebens besteht.

Das ist ein bisschen ambivalent, finde ich. Auf der anderen Seite muss ich sagen, rein vom Gerechtigkeitsempfinden, (...) wenn man sich mit den Mengen beschäftigt, dann ist eine geringe Menge wirklich nicht viel (...). Da muss ich sagen: , Ob das zu bestrafen ist?" (StA 5)

Andere Interviewpartner bestätigten, dass die Grenze zur nicht geringen Menge schnell überschritten werde und dass dieser Strafbarkeitseinschränkung praktisch keine große Bedeutung zukomme:

„In der Tat ist es ja eher so, dass die nicht geringen Mengen äußerst schnell erreicht sind." (StA 7)

„Also in der Regel würde ich sagen, im Kraftsportbereich ist diese nicht geringe Mengel relativ oft überschritten. (...) Da reicht ja praktisch eine 10 ml-Ampulle, also eine Durchstechampulle (...), um dann den Bereich der Strafbarkeit zu eröffnen." (StA 3)

112 BGH StV 2018, 302. 
„Es ist relativ selten, dass jemand so wenig hat, dass es straffrei ist. (...) In der Regel haben die Leute mehr, weil sonst macht es auch faktisch nicht so einen Riesensinn, um damit zu dopen." (StA 4)

„Also, die nicht geringe Menge ist für uns gar kein Problem. Die nicht geringe Menge ist so niedrig festgesetzt nach dem Antidopinggesetz, dass wir ja immer im Bereich der Straftaten sind. Man muss eher dazu sagen, sie ist manchmal so gering, dass schon kleinste Mengen zu einer Straftat fübren (...). “ (Zoll 1)

Lediglich ein Staatsanwalt gab an, dass die Berechnung, ob ein Dopingmittel in nicht geringer Menge vorliegt, bisweilen schwierig sei:

"Wenn das alles nachberechnet werden muss, ist das durchaus aufwendig für die Staatsanwaltschaften. "(StA 1)

Werde etwa nur eine einzelne Ampulle aufgefunden, bestehe zwar ein Anfangsverdacht nach $\$ 2$. Ob ein Verstoß aber tatsächlich vorliege, hänge davon $\mathrm{ab}$, wieviel Wirkstoff in der Ampulle noch enthalten sei. Das müsse man genau berechnen:

"Weil man ja nicht einfach feststellen kann, da hat man eine Ampulle aufgefunden, also liegt ein Verstoß vor, sondern man muss genau berechnen, wieviel ist da noch drin. Oftmals muss man entsprechende Gutachten einholen, vielleicht auch schon wegen der Füllmenge." (StA 1)

Letztlich bestätigt aber auch diese Stimme, dass die Schwellenwerte für nicht geringe Mengen so niedrig angesetzt sind, dass bereits der Fund einer (nicht ganz gefüllten) Ampulle Anlass für Ermittlungen biete. Strafrechtlich verfolgt werden damit vor allem Selbstnutzer, die Dopingmittel für sich selbst verwenden und diese - anders als es die Gesetzesbegründung vermutet - nicht handeln, veräußern oder an andere weitergeben wollen. Häufig richten sich die Verfahren gegen Bodybuilder, die nicht an Wettkämpfen teilnehmen, also nicht dopen, um sich Vorteile im sportlichen Wettbewerb zu verschaffen, sondern aus anderen (etwa: ästhetischen) Gründen.

(3) Verbringen in den Geltungsbereich und durch den Geltungsbereich

Das neue Verbot des Verbringens in den Geltungsbereich dieses Gesetzes ermöglicht es, Dopingmittel bereits an der Grenze der Bundesrepublik 
Deutschland bei der Einreise sicherzustellen ${ }^{113}$ und als Straftat zu ahnden. Unter Strafe steht zudem das Verbringen von Dopingmitteln durch den Geltungsbereich.

Zwei Staatsanwälte äußerten sich zu diesen Tathandlungsvarianten positiv. Die "Verbringungstatbestände" seien "gewinnbringend für uns gewesen" (StA 1) bzw. "ganz entscheidend" (StA 7). Begründet wird dies damit, dass die im früheren Recht vorausgesetzte „Einfuhr“ nur vorgelegen habe, wenn die Ware in den zollrechtlich freien Verkehr - also hinter die Zollkontrolle - verbracht werde. Geschah dies nicht, weil die Ware nicht nach Deutschland geliefert, sondern hier nur umgeschlagen und dann weiter transportiert wurde, fehlte es an einer strafbegründenden Einfuhr. So konnten Strafbarkeitslücken entstehen:

„Verbringen ist letztlich ein zollrechtlicher Begriff, der aber erforderlich ist, weil vorher im Arzneimittelrecht (...) bei den einschlägigen Normen nur von Einfuhr die Rede war. Einfuhr setzt aber den Übergang eines Präparats in den zollrechtlich freien Verkehr voraus, der in der Regel nicht erfolgt, wenn die zollrechtliche Kontrolle dieses Präparat entdeckt. Da damals im AMG keine Versuchsstrafbarkeit bei einigen dieser Normen gegeben war, die die Einfuhr vorsahen, führte das zu einer Straflosigkeit. Die Übernahme des Tatbestands des Verbringens von Dopingmitteln in nicht geringer Menge hat diese Gesetzeslücke geschlossen." (StA 7)

Zur praktischen Bedeutung sagten die Interviewpartner des Zolls:

„Wenn ich eine Ware von China, sage ich, mal nach Polen schicke und es wird am Frankfurter Flughafen kontrolliert, ist sie verbracht. Das heißt, straftatbestandsmäßig ist sie dann, wenn sie durchgeführt wird. Das hat bei uns ganz große Auswirkungen gebabt. (Zoll 1)

„Das ist eine Klarstellung und das hat auf jeden Fall sehr geholfen." (Zoll 3)

Die Vorschrift ist nach Auskunft zweier Interviewpartner des Zolls praktisch von erheblicher Relevanz:

„Ansonsten haben seitdem vor allen Dingen die sog. Durchfuhren an Ermittlungsverfahren zugenommen, also das sind einfach mehr geworden." (Zoll 3)

113 Dazu und zum Folgenden BT-Drs. 18/4898, S. 25; Lehner/Nolte/Putzke/Striegel, Anti-Doping-Gesetz, $\mathbb{2}$ Rn. 83 f. 
"Wir haben eine Vielzabl von Durchfuhrverfahren größter Mengen an Dopingmitteln, die wir im Rahmen der Durchfuhr, sprich des Verbringens jetzt sicherstellen, und auch Strafverfahren einleiten, die dann zum Teil (...) im europäischen Ausland fortgeführt werden (...). "(Zoll 1)

Ein anderer Ermittler wies indes darauf hin, dass es gerade hier schwierig sei, zulässige Formen des Verbringens in oder durch den Geltungsbereich von strafbaren Handlungen zu unterscheiden, da das erforderliche Tatbestandsmerkmal „zum Zweck des Dopings beim Menschen im Sport" nicht den Stoffen selbst anhafte, sondern erst durch weitere Ermittlungen festzustellen sei. Der Dopingzweck sei „manchmal ein bisschen schwierig zu erkennen“. Das betreffe auch „mal größere Sendungen von Arzneimitteln“ (Zoll 2). In solchen Fällen können transnationale Ermittlungen notwendig sein:

„Wenn dann so etwas hier durchgeführt werden soll, zu einem Empfänger, der in einem Drittland sitzt, dann muss der Fahnder die entsprechenden Ermittlungen führen, um Licht ins Dunkel zu bringen, was die Intention des Ganzen gewesen sein kann." (Zoll 2)

Während die Verbringungsverbote für den Zoll eine erhebliche praktische Bedeutung haben, spielen sie für die Strafjustiz bislang kaum eine Rolle. Ein befragter Richter hatte keine Erfahrungen mit Anklagen wegen verbotener Durchfuhr, was auf eine hohe Einstellungsquote hindeutet:

„Also Durchfubr hatten wir noch nie. Wir haben in hohem Maße Herstellen und Handeltreiben gehabt." ( $R$ 1)

Die Verbringungsverbote scheinen daher gegenwärtig vor allem den Zweck zu erfüllen, verdächtige Lieferungen zu prüfen und Dopingmittel sicherzustellen.

ee) Zum Zweck des Dopings beim Menschen im Sport

Die Normen des $\ 2$ und die darauf Bezug nehmenden Straftatbestände des $\$ 4$ Abs. 1 knüpfen die Verbote bzw. die Strafbarkeit an die Verwendung der Dopingmittel bzw. -methoden an den „Zweck des Dopings beim Menschen im Sport". ${ }^{114}$ Unter den Begriff des Sports soll dabei jede sportliche Betätigung fallen; neben dem Leistungs- und Wettkampfsport also ebenso

$114 \$ 2$ Abs. 2 spricht vom Zweck des Dopings im Sport „bei einer anderen Person“. 
der Freizeit- und Breitensport. ${ }^{115}$ Damit wird auch die Leistungssteigerung im Bereich des Bodybuildings durch unnatürlichen Masse- und Kraftzuwachs erfasst. ${ }^{116}$

Während die Einordnung einer Tätigkeit unter den weit gefassten Begriff des Sports keine praktischen Schwierigkeiten bereitet, ${ }^{117}$ kann sich der Nachweis gerade eines Dopingzwecks als schwierig gestalten. Dies betrifft zum einen die Fälle der Verbringung von Arzneimitteln durch den Geltungsbereich (dazu vorstehend dd. (3)) und zum anderen die bereits oben (unter 1.a.) erwähnten Lifestyle-Präparate. Diesbezüglich deutet die Untersuchung darauf hin, dass ein Hauptgewicht der Ermittlungen auf der Frage liegt, ob das Präparat als Dopingmittel „zum Zweck des Dopings beim Menschen im Sport“ dienen soll oder aus anderen - zulässigen - Motiven eingesetzt wird.

„Im Moment haben wir da so ein Präparat, das hier sehr vermehrt vorkommt, und zwar ist das DHEA (Dehydroepiandrosteronacetat). (...) Es ist so ein Lifestyle-Präparat, das auch so ein Heilsversprechen hat, für das es in den USA eine Zulassung gibt und das auch hier z.T. durch Ärzte in Deutschland verschrieben wird. (...) [D]as kann gegen Krebsleiden und Antistress und Anti-Aging eingesetzt werden und die Alterung der Zellen soll damit aufgehalten werden, die ganze Bandbreite; auch zu Wechseljahresstörungen bei der Frau (...) [D]ann taucht das hier überwiegend auf. (...) Das macht hier eine Heidenarbeit, man kommt hier dem grundsätzlichen Auftrag des AntiDopG nach, aber es ist in dem Bezug vielleicht jetzt nicht so zielführend. Man sieht, dass die Leute (...) in ein Ermittlungsverfahren hineinkommen können, die durch eine andere Intention (...) sich ein Arzneimittel bestell haben, meistens auch noch in Unwissenheit, dass da eine Arzneimitteleigenschaft vorliegt." (Zoll 2)

Wenn bei der Einreise Personen mit derartigen Präparaten angetroffen werden, scheinen die Ermittlungen durch eine Inaugenscheinnahme und Nachfragen zu erfolgen. Ein Ermittler schilderte beispielhaft, dass sich die Kolleginnen und Kollegen dann fragten:

„Hat man einen athletischen Menschen vor sich? (...) Was gibt er so von sich? Wenn die Person jetzt vor mir im Reiseverkehr stehen würde und ich

115 Körner/Patzak/Volkmer/Volkmer, BtMG, 9. Aufl. 2019, AntiDopG $\$ 4$ Rn. 24; Erbs/Kohlhaas/Wußler, 228. EL Januar 2020, AntiDopG \$2 Rn. 6.

116 BGH NStZ 2010, 170.

117 In der Literatur umstritten ist gegenwärtig vor allem die Frage, ob auch der sog. E-Sport als Sport zu verstehen ist, siehe dazu Kubiciel, ZRP 2019, 200, 203. 
hätte einen siebzigjäbrigen Rentner mit DHEA, dann frage ich ibn: Wofür ist das gedacht? Sagt er: Das ist für mich, ich komme gerade aus den USA, ich habe (gesundheitliche) Probleme und dafür nehme ich das ein. Dann (...) habe ich überhaupt keinen Anfangsverdacht einer strafbaren Handlung. Sie können es behalten, das AntiDopG ist raus. Die Arzneimittel im Reiseverkehr haben sie als Dreimonatsbedarf. Wenn der 100 Tabletten mitbringt, sage ich: ,Komm, eine am Tag sind 92, ich drück mal die Augen zu, nimm die 100 mit, schönen guten Tag noch!' Und dann lasse ich den Mann ziehen. "118

Nicht immer ist eine solche erste Einschätzung durch eine Inaugenscheinnahme möglich. Dann gestalten sich die Ermittlungen aufwändiger und binden Ermittlungsressourcen, insbesondere weil nicht wenige Beschuldigte sich offenbar in Internet-Foren über Verteidigungsmuster austauschen, um darzulegen, dass das Präparat nicht zum Zweck des Dopings im Sport verwendet wird:

„Was dann (...) vom Beschuldigten kommuniziert wird, da fragt man sich manchmal, welche Foren er angelesen hat, was er vielleicht sagen müsste, um da vielleicht mit einem blauen Auge rauszukommen." (Zoll 2)

ff) Fahrlässigkeitsstrafbarkeit nach $\$ 4$ Abs. 6


fahrlässige Begehung der Straftaten nach Absatz 1 Nr. 1-3. Der Gesetzgeber begründet die Kriminalisierung damit, dass es sich „um Gesundheitsgefährdungstatbestände“ handle, indem er $₫ 4$ Abs. 1 Nr. 1-3 mit so unterschiedlichen Tatbeständen wie der fahrlässigen Brandstiftung ( $\$ 306 \mathrm{~d}$ StGB), der Trunkenheit im Verkehr ( $\$ 316$ StGB) und der fahrlässigen Körperverletzung ( $\mathbb{2 2 9}$ StGB) vergleicht. Die Anordnung einer Fahrlässigkeitsstrafbarkeit sei daher „sachgerecht“. Relevant soll die Fahrlässigkeitsstrafbarkeit vor allem in Fällen sein, in denen der Täter nicht erkannt hat, dass es sich bei der jeweiligen Substanz um ein Dopingmittel im Sinne dieses Gesetzes handelt. ${ }^{119}$

118 Hier wurde auf eine Bezeichnung des Interviewten verzichtet, um keine Rückschlüsse auf dessen Identität zu ermöglichen.

119 BT-Drs. 18/4898, S. 25; Graf/Jäger/Wittig/Eschelbach, Wirtschafts- und Steuerstrafrecht, $\mathbb{} 4$ AntiDopG Rn. 34. 
Quantitativ betrachtet scheint die Bedeutung von $\$ 4$ Abs. 6 indes äuBerst gering zu sein.

„Wir hatten einmal eine Verurteilung wegen fahrlässiger Begehensweise. (...) Ein Fall, das ist jedenfalls die zablenmäßige Bedeutung." (R 1)

„Die Fahrlässigkeitstatbestände sind bei mir noch nicht groß in Erscheinung getreten." (StA 5 ebenso StA 1)

Als Grund wird dafür genannt, dass ein fahrlässiger Erwerb oder Besitz von Dopingmitteln zum Zweck des Dopings beim Menschen kaum vorstellbar seien.

„Das hängt damit zusammen, dass man ja, ich sag jetzt mal so, wie man fahrlässig an solche Produkte kommen soll, ist auch etwas schleierhaft. Also wenn man solche Produkte bestellt, dann tut man das meistens mit einem gewissen Hintergrund. "(StA 5)

Zudem berichteten die Befragten, dass in Fällen, in denen eine fahrlässige Begehung im Raum steht, meist von einer geringen Schuld des Täters ausgegangen und das Verfahren eingestellt wird. $\$ 4$ Abs. 6 spiele daher ,praktisch keine große Rolle, weil in solchen Fällen in der Regel mit $\$ 153 \mathrm{ff}$. im Bereich der Opportunitätsentscheidung gearbeitet wird. "(StA 3)

Dennoch wird die Existenz der Fahrlässigkeitstatbestände aus unterschiedlichen Gründen begrüßt. Ein Staatsanwalt nennt einen symbolischkommunikativen Grund:

„Also in der Praxis haben sie meiner Auffassung nach bislang keine große Rolle gespielt. (...) Gleichwohl halte ich sie für ein wichtiges Zeichen, ja dass man einfach auch klarstellt, dass eben das auch unter Strafe steht." (StA 1)

Zwei andere Staatsanwälte sehen die Fahrlässigkeitstatbestände als notwendige Auffangtatbestände für Fälle, in denen der Vorsatz nicht nachweisbar ist:

„Sicherlich ist der Abs. 6 aber sehr wichtig als Auffangtatbestand."(StA 2)

„Also sie sind wichtig, weil eine Fahrlässigkeitsstrafbarkeit muss immer geregelt werden, weil es schon Fälle gibt, in denen Menschen keinen bedingten Vorsatz haben (...)." (StA 3)

Ein anderer Staatsanwalt hält dem entgegen, dass ein Auffangtatbestand in der Praxis unnötig sei, da es "meistens auf einen bedingten Vorsatz hinausgelaufen" sei. (StA 2) 
b) Qualifikationstatbestände

\4 Abs. 1 sieht für Verstöße gegen die Verbote des $₫ 2$ Freiheitsstrafe bis zu drei Jahren oder Geldstrafe vor. Aufbauend auf diesen Grundtatbestand schafft $₫ 4$ Abs. 4 Qualifikationstatbestände, für die ein deutlich erhöhter Strafrahmen von Freiheitsstrafe von einem Jahr bis zu zehn Jahren gilt. Es handelt sich um Verbrechen im Sinne des $\$ 12$ Abs. 1 StGB. Die Erschwerungsgründe sollen besonders verwerfliche und sozialschädliche Verhaltensweisen erfassen. ${ }^{120}$ Sie entsprechen inhaltlich den Regelbeispielen besonders schwerer Fälle im bisherigen $\$ 95$ Abs. 3 Nr. 1 und $\$ 2$ AMG, werden aber um die Veräußerung der Dopingmittel an eine Person unter 18 Jahren, die Verschreibung eines Dopingmittels an sowie die Anwendung einer Dopingmethode bei einer solchen Person ergänzt. Für eine Umgestaltung in einen Qualifikationstatbestand macht die Gesetzesbegründung geltend, dass „praktisch keine Fälle denkbar (sind), in denen trotz Vorliegens der jeweiligen Voraussetzungen eine Strafschärfung nicht angemessen erscheint." 121

In der Praxis wird die Hochstufung begrüßt, nicht nur wegen der praktischen Folgen, sondern auch wegen der symbolischen Wirkung:

„Ja gut, das hat insofern Auswirkungen auf die Praxis, dass man da tatsächlich Verbrechenstatbestände drin hat und dass man im Prinzip über diese Schiene auch dem Doping auch so ein bisschen den Charakter vom Kavaliersdelikt genommen hat, weil es jetzt tatsächlich mehr ans BtMG gerückt ist (...)." (StA 5)

„Ich glaube, besonders begrüßenswert ist beim $\$ 4$ Abs. 4 die Hochstufung der Gewerbsmäßigkeit zum Verbrechenstatbestand. Das erlebt man auch immer wieder bei Gericht, wenn man dann verdeutlicht. Oftmals ist es ja nicht allen Verfahrensbeteiligten so klar auf die Schnelle, wenn man es dann noch einmal deutlich macht, das ist jetzt ein Verbrechenstatbestand, dann zeigt es einfach, wie der Gesetzgeber doch durchaus ja entsprechende Vorschriften aufgewertet hat, wie ja auch eben die Doping-Strafbarkeiten entsprechend hier höher bewertet und eben dieser Aspekt, den halte ich für sehr sinnvoll, ja. " (StA 1)

120 BT-Drs. 18/4898, S. 31; Weber BtMG/ders., 5. Aufl. 2017, AntiDopG $₫ 4$ Rn. 282; Graf/Jäger/Wittig/Eschelbach, Wirtschafts- und Steuerstrafrecht, 2. Aufl. 2017, AntiDopG $\$ 4$ Rn. 35.

121 BT-Drs. 18/4898, S. 30. 
Auch mit Blick auf die Verjährung wird die Regelung für notwendig erachtet:

„Die Verbrechenstatbestände - das spielt schon eine große Rolle (...), weil ja dadurch die Verjährungsfristen verlängert worden sind. Jetzt im Bereich 4 Abs. 4 AntiDopG haben die Verjährungsfristen ja zehn Jahre, während sie unter dem Bereich $\$ 95$ AMG damals noch fünf Jahre waren als besonders schwerer Fall." (StA 3)

Zudem wird auf die Möglichkeit der Telekommunikationsüberwachung als einer notwendigen besonderen Ermittlungsmaßnahme verwiesen:

„Die Gewerbsmäßigkeit oder als Mitglied einer Bande fortgesetzt oder Abgabe an Jugendliche. Das war schon früher im AMG auch Strafnorm. Es ist jetzt natürlich klarer und definierter, insbesondere auch die Möglichkeit, über $\$ 4$ Abs. 2 Nr. 2 dann auch in den $\$ 100 a$ [StPO] zu bekommen und dann auch leichter eine Telefonüberwachung zu bekommen. Das sind die Vorteile bei uns. Bei der Strafbarkeit und bei der Aburteilung hat sich da nicht viel geändert." (Zoll 1)

Die Hochstufung der bisherigen Regelbeispiele zu Verbrechenstatbeständen hat darüber hinaus zur Folge, dass die in diesem Gesetz genannten Straftaten beim Vorliegen der qualifizierenden Voraussetzungen dem Vortatenkatalog des Geldwäschetatbestands des $₫ 261$ Abs. 1 S. 2 StGB unterfallen. Dieser Aspekt scheint sich bislang allerdings kaum auszuwirken; die befragten Praktiker wussten von keinen Fallbeispielen zu berichten. Dies könnte auch daran liegen, dass der Großteil der Verfahren gegen Selbstnutzer geführt wird, bei denen Anschlussstraftaten wie eine Geldwäsche praktisch ausscheiden. Ein Ermittler deutete noch einen anderen Grund für diesen Befund an:

„Das ist ein großes Problem. Die Geldwäsche, das Abschätzen der Gewinne bereitet uns immer noch Schwierigkeiten in diesem Deliktsbereich, da wir nachweisen müssen, zu welchen Preisen die Rohstoffe im Ausland erworben wurden, mit welcher Gewinnspanne veräußert wurden." (Zoll 1)

Hinzu treten Ermittlungsschwierigkeiten, etwa wenn Social-Media-Kanäle zur Kommunikation genutzt werden:

„Das hat aber was damit zu tun, dass es sebr schwierig ist, wenn jemand nur per WhatsApp kommuniziert und die Absprachen, auch der Handel oft ja bei der Übergabe in Fitnessstudios läuft, dass da nichts verschriftet und es uns sehr schwerfällt, letztendlich eine Vermögensabschätzung nachher durchzufübren." (Zoll 1) 
Ein Staatsanwalt dachte darüber nach, ob nicht in Anlehnung an das BtMG auch ein Handeltreiben mit Waffen ein Qualifikationstatbestand sein solle:

„Was zu bemerken ist, es ist eine Sache im Unterschied zum BtMG: Es fällt tatsächlich auf, dass das Handeltreiben mit Waffen nicht unter Strafe gestellt ist (...) Dafür sind ja tatsächlich im Betäubungsmittelstrafrecht nicht unter fünf Jabren angesetzt, während es das hier nicht gibt. "(StA 5)

Auf die Frage, ob es für die Einführung eines solchen Qualifikationstatbestandes ein praktisches Bedürfnis gebe, antwortete der jedoch eher verneinend:

„Das ist die große Frage. (...) Die größten Vertriebswege sind im Kern die Fitnessstudios und Privatverkäufe im Internet. Also es ist die Frage, ob es einen Anwendungsbereich gäbe." (StA 5)

c) Zusammenfassung

Die Umgestaltung und Erweiterung der AMG-Tatbestände in Anlehnung an das BtMG werden überwiegend begrüßt. $\mathrm{Zu}$ vernehmen waren hingegen Zweifel an der Begründung der Erwerbs- und Besitzstrafbarkeit, zumal die Schwellenwerte für nicht geringe Mengen offenbar so niedrig sind, dass sie in aller Regel überschritten werden. Damit werden in großem Ausmaß auch Selbstnutzer kriminalisiert, bei denen die Absicht der Weitergabe von Dopingmitteln an Dritte nicht ohne weiteres vermutet werden kann. Das Verbot der Verbringung in oder durch den Geltungsbereich des Gesetzes wird weithin für gelungen erachtet. Schwierig scheint gerade in diesen Fällen aber der Nachweis des Dopingzweckes zu sein. Für richtig erachtet werden ferner die Einführung der Qualifikationstatbestände des Abs. 4 sowie die Erfassung fahrlässigen Handelns, wobei die Fahrlässigkeitstatbestände in der Praxis nur selten relevant werden.

3. Erkenntnisse zu prozessualen Fragen

Den Sachverständigen lagen für ihre Bewertung keine Akten vor; die Evaluation der prozessualen Fragen stützt sich daher auf die geführten Experteninterviews. 
a) Anfangsverdacht

Die Begründung eines Anfangsverdachts einer Straftat nach $\$ \mathbb{S} 4$ Abs. 1 Nr. 1-3 i.V.m. $\$ 2$ hängt naturgemäß von der konkreten Tatalternative ab. Dennoch lassen sich drei typische Situationen bzw. Anlasssituationen für die Begründung eines Tatverdachts nennen: das Auffinden mutmaßlicher Dopingmittel bei Zollkontrollen und im internationalen Post- und Warenverkehr; Ermittlungen gegen Vertriebsstrukturen und Handel im Internet, namentlich im sog. Darknet sowie Zufallsfunde, etwa bei Ermittlungen, die gegen andere Personen geführt werden.

Im Bereich des Zolls dominieren Funde bei der Einfuhrkontrolle sowie in Postverteilzentren:

„Also da wir hier auch zuständig sind für den Frankfurter Flughafen, aber auch für verschiedene Postverteilzentren ist unser Hauptaugenmerk natürlich die Einfuhrkontrolle und bei dieser Einfuhrkontrolle wird im Rabmen von körperlicher Kontrolle aber auch Röntgenkontrolle, Papierkontrolle festgestellt, dass Stoffe nach Deutschland gelangen, die verboten sind, sprich unter das Antidopinggesetz fallen. Die sind falsch deklariert oder versteckt oder es wird was verschleiert. " (Zoll 1)

„In der Regel hat sie der Endkonsument dabei, ganz normal im Gepäck oder im Auto, je nachdem, wo die Kontrollen stattfinden. Ansonsten kommen sie bei Bestellern im Internet oder Darknet mit der Post, in Brief-oder Paketsendungen oder auch Einschreibesendungen. " (Zoll 3)

Hinzu treten Informationen, die aus Ermittlungen gegen Vertriebsstrukturen und gegen Anbieter von Dopingmitteln im Internet gewonnen werden. Hier entstehen Ermittlungsansätze etwa, „wenn eine Vertriebsstruktur zerschlagen wurde, ein Server gespiegelt oder ein Untergrundlabor ausgehoben wurde. Nach Auswertung z.B. der elektronischen Speichermedien ergeben sich Abnehmerverfahren von den Präparaten. " (Zoll 3)

Auch ein Staatsanwalt einer Schwerpunktstaatsanwaltschaft bestätigte, dass „(...) insbesondere aus den Bestellisten aus dem Internet oder auch aus Darknet-Ermittlungen" (StA 1) weitere Ermittlungsverfahren generiert werden.

Schließlich sind „es natürlich auch Zufallsfunde“ (StA 1), also Anhaltspunkte für Verstöße gegen das AntiDopG, die im Zuge anderer Ermittlungen gewonnen werden:

„Also ganz oft haben wir auch entsprechende Verfahren, dass Beschuldigte, bei denen durchsucht wird wegen irgendeinem anderen Delikt und dass man 
dabei dann diverse Dopingmittel auffindet und so ein Verfabren einleitet." (StA 1)

In Bezug auf das Handeltreiben und Herstellen scheint der Anfangsverdacht oft aus einer Übersendung abgeleitet zu werden, sobald der Schwellenwert einer nicht geringen Menge überschritten wird:

„Wenn illegale Substanzen, Präparate oder Wirkstoffe aufgefunden werden, sei es beim Verbringen aus anderen EU-Mitgliedstaaten oder eben direkt aus dem Drittland, so dass sich dann, zumindest aufgrund der Überschreitung der nicht geringen Menge oder ansonsten auch direkt der Verdacht auf Handeltreiben oder Herstellung von Dopingmitteln ergibt. " (Zoll 3)

b) Ermittlungsmaßnahmen

Die Experteninterviews haben gezeigt, dass die Ermittler und Staatsanwälte häufig einem standarisierten Ermittlungsschema folgen. Dies gilt vor allem für Ermittlungen beim Zoll, die in der Regel wegen ähnlich gelagerter Sachverhalte eingeleitet werden. Hier werden einzelne Ermittlungsschritte bereits „vor Ort“ vollzogen, bevor die Staatsanwaltschaft einbezogen wird:

„Überwiegend sind das Aufgriffe bei uns im internationalen Postverkehr, d.h. Postsendungen mit kleineren Mengen von Dopingmitteln, die aufgrund von Risikokontrollen uns dann sozusagen vorgeführt werden. Die Kollegen öffnen das Ganze, schauen sich das an, bewerten die aufgefundenen Präparate nach Rechtsbereichen. Zum Beispiel haben wir jetzt hier Präparate vorliegen, die dem Arzneimittelgesetz unterliegen oder sind jetzt auch Präparate dabei, die auch die Liste nach der Dopingmittel-Mengen-Verordnung aufweist? Dann sind wir im AntiDopG. Dann wird die Berechnung vorgenommen, ob die nicht geringe Menge überschritten wurde, weil sich ja vom Grundsatz her der Verdacht einer strafbaren Handlung nach dem AntiDopG ergeben könnte, weil solche Dopingmittel widerrechtlich in die BRD verbracht wurden und theoretisch auch zur Anwendung im Sport herangezogen werden könnten. Dann wird die Sendung sozusagen strafvollzugshalber sichergestellt. Das ist eine Tätigkeit, die der Abfertigungsbeamte vor Ort dann macht. Dann geht es zu uns zur weiteren Sachbearbeitung weiter. Wir prüfen dann, ob das, was da gemacht wurde, also nochmals so eine Art Qualitätskontrolle, machen dann von hier eine schriftliche Anbörung. Das erfolgt dann auch in Absprache und im Auftrag der StA Frankfurt/Main [...] und wir geben dann dem Beschuldigten die Möglichkeit, zum Tatvorwurf, der ihm jetzt erstmal gemacht wird, im Rahmen des Ermittlungsverfahrens 
Stellung zu beziehen. Dann warten wir die entsprechende Rückmeldung ab oder auch nicht, ergänzen das Ganze mit den personenbezogenen Daten und machen das Verfahren dann bei der StA anhängig. " (Zoll 2)

Ausgangspunkt sind in vielen Fällen, in denen Dopingpräparate aufgefunden werden, Befragungen bzw. Anhörungen, die der Ermittlung des Verwendungszwecks dienen. Parallel dazu werden Internet-Recherchen oder Datenbankabfragen durchgeführt, um Verdachtsmomente zu prüfen oder Antworten des Beschuldigten in der schriftlichen Anhörung auf Plausibilität zu prüfen:

„Was meine Tätigkeit mit sich bringt mit diesen Kleinfällen ist immer dieser Weg der schriftlichen Anbörung. (...) Dann kann man auch einfach mal durch Internet-Recherchen oder Datenbankabfragen einmal versuchen, für den Fall Licht ins Dunkel zu bringen. Wenn man sieht, da passt irgendetwas nicht, ist das immer so ein bisschen vom Timing desjenigen abhängig, der den Vorgang der Tat aufdem Tisch hat oder was sich da auch manchmal daraus ergibt." (Zoll 2)

Danach entscheiden die Ermittler in Absprache mit der Staatsanwaltschaft das weitere Vorgehen:

„Ermittlungen in Form einer schriftlichen Anhörung von uns. Alles weitere, das ermittlungstaktisch im Raum stehen könnte, entscheidet die Zollfahndung sozusagen verhältnismäßig oder in Absprache mit der StA. " (Zoll 2)

Als häufigste Ermittlungsmaßnahme nach der Anhörung des Betroffenen wurde die Hausdurchsuchung genannt:

„Also regelmäßig zur Aufklärung solcher Fälle machen wir auf jeden Fall Durchsuchungen, Sicherstellungen und Beschlagnahmungen werden durchgeführt."(Zoll 1)

"Klar, die erste Maßnahme ist natürlich die Hausdurchsuchung. Da finden wir eigentlich immer etwas; wenn ein Anfangsverdacht besteht, mit sehr großem Erfolg. " (StA 2)

Andere Ermittlungsmaßnahmen werden seltener genannt und offenbar auch nur in Ausnahmefällen angewandt:

„Telefonüberwachungsmaßnahmen, klar, die gibt es auch in wenigen Fällen, sage ich jetzt mal, da findet man dann natürlich auch irgendwelche Abnehmer, die sich telefonisch beim jeweiligen Täter melden. Da hat man dann wieder neue Ermittlungsansätze. Im Rahmen des neuen Vermögensabschöpfungsrechts gibt es natürlich die Möglichkeit der Arrest-Pfändungsbe- 
schlüsse, was ich schon gemacht hatte, um einfach den Gewinn abzuschöpfen. Das sind so die meisten Zwangsmaßnahmen." (StA 2)

„Wenn man sich im Bereich des $\$ 4$ AntiDopG, also im Verbrechensbereich bewegt, dann sind diese dortigen Delikte in aller Regel Katalogtaten im Sinne der 100aff. der StPO, die uns dann erlauben, mit irgendwelchen Maßnabmen dann auch erst einmal operativ einzusteigen. Da werden dann Telefone überwacht, E-Mail-Accounts ausgelesen, da werden Observationen durchgeführt, Pakete beschlagnahmt. Da werden möglicherweise im Auto oder außerhalb von Wohnungen Abhörvorrichtungen installiert und ausgewertet." (StA 3)

Die Ermöglichung der TKÜ wird als Verbesserung der Ermittlungsmöglichkeiten empfunden, da der Tatnachweis bei Ermittlung wegen gewerbsmäßigen Handeltreibens im Untergrund schwer zu führen ist:

"Ja, natürlich, bei Großverfahren braucht man keine Hilfsbrücke mehr suchen, wie man es früher musste, sondern wir haben jetzt eine Strafnorm, die im $\ 100 a$ erwähnt ist. Das führt natürlich dazu, dass wir bei gewerblichen Fällen, das sind ja meistens die großen, dann wirklich auf die TKÜ-Maßnabmen zurückgreifen können." (Zoll 1)

„Gewerbsmäßiges Handeltreiben mit Dopingmitteln, da hat sie unsere Ermittlungsmöglichkeiten durchaus erweitert und durchaus greifen wir sehr gerne darauf zurück vor dem Hintergrund eben, gerade dass Labore oder Untergrundhandel sonst auch sehr, sehr schwierig festzustellen ist." (StA 1)

Als größte Schwierigkeit in der Beweisführung wurde der Zugriff auf Inhalte von Messenger-Diensten genannt:

"Ja, die Schwierigkeit in der Beweisführung ist zum einen, dass ganz viel (...) mittlerweile über Messenger-Dienste läuft. Das heißt, über WhatsApp und andere Dienste, die für uns nicht zu überwachen sind. "(Zoll 1)

Hinzu kommt die Kommunikation in geschlossenen Internet-Foren und für Dritte unzugänglichen Chatrooms:

„Zum anderen findet Kommunikation in Chatrooms, also abgeschotteten Chatrooms statt, zu denen wir keinen Zugang haben." (Zoll 1)

Schließlich wurden die Möglichkeiten der Verschlüsselung und Löschung von E-Mails als Ermittlungshindernis betont:

„Man bedient sich zum Beispiel Programmen, die eine Mail, nachdem sie abgeschickt ist, unmittelbar löscht." (Zoll 1) 
Häufiger erwähnten die Ermittler des Zolls, dass sie regelmäßig transnationale Ermittlungen durchführen. Auffällig ist dabei, dass die Zusammenarbeit mit den Behörden anderer EU-Staaten standardisiert zu sein scheint und offenbar ohne größere Schwierigkeiten verläuft:

„Es gibt da dieses Medium der Spontanmitteilung. Sie müssen sich das so vorstellen. Ich bekomme jetzt gleich eine E-Mail vom Frankfurter Flughafen, dass $50 \mathrm{~kg}$ Testosteron versteckt in Fässern sichergestellt wurden und der Empfänger sitzt irgendwo in Frankreich und der Versender aus China. Am Frankfurter Flughafen ist es aufgefallen. Daraufhin habe ich ja alle Daten vorliegen, fertige unmittelbar eine Spontanmitteilung übers Zollkriminalamt an die französischen Kollegen, so dass die noch am gleichen Tag zum Teil, wenn nicht am nächsten Tag in die Lage versetzt werden, Maßnahmen $z u$ treffen oder das Verfahren anzuziehen oder aber auch zu sagen, wir machen eine kontrollierte Lieferung dieser Sendung weiter nach Frankreich, damit die feststellen können, wer eigentlich dort Empfänger ist und dann eventuell auch strafprozessual dort vorgehen können." (Zoll 1)

„In der Regel werden diese Erkenntnisse über Absender, Empfänger und Menge an den Empfangsstaat mitgeteilt, zum einen, ob Interesse an der Übernahme des Verfahrens besteht, und zum anderen, um z.B. die Person zu identifizieren, weil eine Staatsanwaltschaft mehr Punkte bekommt für ein Strafverfahren gegen bekannte Personen als gegen unbekannte Personen. Ansonsten werden Sachverhalte übermittelt über unsere Rechts- und Amtshilfeabteilung, Zentralstelle im Rahmen von Neapel-II-Ersuchen, das ist von Zoll zu Zoll, aber auch auf der Polizeischiene und vereinzelt auch über Europol." (Zoll 3)

Ein Problem scheint hingegen zu sein, dass Rechtshilfe-Ersuchen, die an Nicht-EU-Staaten gerichtet werden, oft am Fehlen einer gegenseitigen Strafbarkeit scheitern, da der in Rede stehende Wirkstoff im Ausland frei verkäuflich ist und kein Dopingmittel darstellt oder gar kein dem $\ 2$ entsprechendes strafbewehrtes Verbot existiert:

„Man muss klar sagen, dass es in den Ländern außerhalb der EU oftmals so ist, dass die Sachen, die bei uns unter das Antidopinggesetz fallen, dort nicht unbedingt mit Strafe bedroht sind. Das heißt, nicht jeder Dopingstoff, den wir hier wegen Doping sicherstellen, würde auch dort im Ausland überhaupt $z$ u einem Strafverfahren führen, sondern ist vielmehr frei verkäuflich." (Zoll 1) 
c) Abschluss der Verfahren

Die Befragung der Staatsanwälte und Richter ergab keine Besonderheiten beim Abschluss der Verfahren. Wie auch in anderen Deliktsbereichen der leichten und mittleren Kriminalität werden viele der Verfahren eingestellt. Anders als beim Selbstdoping kommt es jedoch auch regelmäßig (und teilweise sogar überwiegend) zu Strafbefehlen und - in schweren Fällen - zu Anklagen.

"Ich sag aber auch und das ist tatsächlich die Realität, es werden relativ viele Verfahren nach \$153 eingestellt." (StA 5)

„Überwiegend enden die mit Strafbefehl und einer Geldstrafe. In Einzelfällen wird auch mal Anklage erhoben, wenn es richtig viel ist oder entsprechende Vorstrafen da sind. Dann enden die oft mit Bewährungsstrafen und viele Verfahren werden auch nach Opportunitätsvorschriften eingestellt, also gegen Zablung einer Geldauflage oder teilweise auch obne Auflage." (StA 6)

Neben einschlägigen Vorstrafen spielt für die Staatsanwälte vor allem die Menge der aufgefundenen Dopingmittel eine maßgebliche Rolle.

"Prinzipiell geht es zuerst mal darum, erstens um das Wievielfache überschreiten die aufgefundenen Dopingmittel die nicht geringe Menge? Und der Faktor der nicht geringen Menge ist für uns tatsächlich der erste Anhaltspunkt, in welche Richtung die Reise geht. Der zweite Faktor ist, ist der Beschuldigte schon mal einschlägig in Erscheinung getreten?" (StA 5)

Eine Einstellung kommt für die Ermittler insbesondere dann in Betracht, wenn die „nicht geringe Menge“ nur unwesentlich überschritten wurde. Hier zeigt sich, dass die Grenze derzeit so niedrig angesetzt wird, dass selbst bei Besitz des Doppelten der geringen Menge ein Handeltreiben als fernliegend angesehen wird.

„Ich habe zum Beispiel jemanden, der hat irgendwie das Zweifache der nicht geringen Menge bestellt. Da muss ich mir schon überlegen (...) gewerbsmäBiges Handeltreiben, halte ich da eher für abwegig. Was kommt da am Schluss raus?" (StA 5)

Die Überführung der Regelbeispiele des AMG in die mit einer Mindestfreiheitsstrafe von einem Jahr bedrohten Qualifikationstatbestände des $\$ 4$ Abs. 4 hat zur Folge, dass Einstellungen aus Opportunitätsgründen in diesen Fällen nicht mehr möglich sind. 
„Die Hauptänderung war möglicherweise, dass wir wegen des höheren Strafrahmens überhaupt mehr solcher Fälle bekommen haben. " $(R 1)$

Gleichwohl gaben die befragten Richter an, dass sie nur selten mit Verfahren nach $\mathbb{} 2$ i.V.m. $\mathbb{} 4$ befasst sind. Dies galt auch für den befragten Richter am Schwerpunktgericht.

Interviewer: „Wie hoch ist denn ungefähr der Anteil der Dopingverfahren an den Gesamtverfahren, die Sie haben?"

$R^{122}$ : $\quad$ "Das ist ein ziemlich kleiner Anteil. Das sind vielleicht $5 \%$, aber mehr ist es auf gar keinen Fall."

Der Richter, der nicht an einem Schwerpunktgericht eingesetzt ist, berichtete von fünf Dopingfällen im Laufe der fünfeinhalb Jahre, in denen er für Dopingverfahren zuständig ist.

Die vor Gericht verhandelten Verfahren enden - wenn es zu einer Verurteilung kommt - in aller Regel mit Geldstrafen; Freiheitsstrafen werden nach Auskunft des Richters nur selten verhängt, insbesondere bei einer Vielzahl einschlägiger Vorstrafen. Auch hier unterscheidet sich die Praxis nicht erkennbar von anderen Deliktsbereichen.

d) Besonderheiten des Gerichtsverfahrens

„Viele Verfahren, die jetzt wirklich zum Gericht durchkommen, sind einfach gelagert. Also das sind oft Strafbefehlsverfahren, wo dann Einspruch eingelegt wird. Dann kommt es zur Hauptverhandlung. In der Hauptverhandlung selbst sind die Beschuldigten oft geständig. Oft wird vorgetragen: "Ja, das war so. Aber warum stehe ich jetzt hier? Ich bin doch kein Verbrecher. Ich tu doch keinem weh." Und dann werden die relativ zügig und einfach durchgeführt.

Größere Verfahren, wenn es um Handel geht, die gestalten sich im Grunde genommen ähnlich wie im Betäubungsmittelverfahren, wobei man sagen muss, dass die Klientel so ein bisschen anders ist. So ein Betäubungsmittelhändler oder entsprechende Verteidiger, die dann da auch oft auftreten, sind sagen wir mal eher Profis. Im Dopingbereich sind das eher Leute, die sind da halt irgendwie drauf gestoßen und haben gedacht, ah, da lässt sich ganz gut mit ein bisschen Geld verdienen und das tut ja keinem weh. Und wenn ich das im Fitness-

122 Eine Nummer wird hier nicht genannt, um Rückschlüsse auf die Antworten des Richters am Schwerpunktgericht auf andere Fragen zu vermeiden. 
studio da meinen Kumpels auch irgendwie besorge, ist das ja kein Problem. Und dann lassen sich diese Verfahren auch vor Gericht eigentlich ganz gut händeln. Also zügig. "(StA 6)

Nach Auskunft der Experten werden vor Gericht ganz überwiegend Verfahren aus dem Bereich des Bodybuildings oder dem Breitensport verhandelt. Tatvorwürfe nach $\$ 2$ stehen damit offenbar selten im Kontext von Doping im Rahmen des Leistungssports.

„Entweder war es der Bodybuilding-Studiobetreiber, der aber selbst das Zeug auch genommen hat und an seine Kunden weitergegeben hat oder die Bodybuilder selber. Da waren dann glaube ich auch ein oder zwei dabei, die das dann en gros bestellt haben und das dann weiterveräußert haben, so dass die dann in den Verbrechenstatbestand reingerutscht sind. Aber im Grunde genommen sind es die typischen Selbstschädiger. Ich warte auch noch drauf, dass der erste, keine Abnung, Leichtathlet oder irgend so etwas kommt, aber bislang..." (R 2)

Mit Blick auf den Ablauf der Verfahren stellten die befragten Experten keine Besonderheiten fest.

„Zu Gerichtsverfahren gibt es keine Besonderheiten. Also es läuft so ab wie bei jedem anderen Verfabren auch. Betäubungsmittel. Da gibt es keine Unterschiede." (StA 5)

Die Verfahren waren in der Regel einfach gelagert und wurden meist zügig erledigt.

"Ja. Also in der Tendenz würde ich sagen, sind das eher einfach gelagerte Verfabren, die sich schnell vor Gericht erledigen. Also mit wenigen Terminen, oftmals mit einem. Dass es da jetzt Verfahren gibt, die lange bei Gericht anhängig sind mit vielen Hauptverhandlungsterminen, kann ich Ihnen aus eigener Erfabrung da gar nichts sagen." (StA 6)

„Die dauern nicht übermäßig lange. Die werden genauso schnell oder langsam abgearbeitet wie alle anderen Deliktsfelder." (StA 7)

Beweisprobleme stellten sich in den Gerichtsverhandlungen überwiegend nicht.

„Nein, also die Verfahren, die wir bisher hatten, die waren auch alle sozusagen unstreitig. Gut, was will man auch bestreiten? Wenn das Zeug bei einem gefunden wird. (...) Und ich kann mich nicht an ein Verfahren erin- 
nern, wo tatsächlich ernsthaft mal überhaupt Beweis erhoben worden wäre. Aber wie gesagt, das liegt natürlich daran, dass, wenn sie dann rein gehen und die Sachen sicherstellen, was will man dann auch bestreiten?" ( $R$ 2)

Aus diesem Grund sind auch Absprachen in der Hauptverhandlung äuBerst selten.

"Also die Mehrzabl der Verfahren ist ja im kleineren Bereich: kleinere Kriminalität, Besitz von Dopingmitteln, da geht es ja immer um eine überschaubare Anzahl von Tagessätzen. Die Beweislage ist klar, die Dopingmittel wurden gefunden, das sind nicht unbedingt Verfahren, die sich zu einer Verständigung eignen. " (StA 4)

„Nein. Wir machen keine Deals in diesen Verfahren." (StA 6)

Allerdings sind häufig Sachverständigengutachten erforderlich, um „die Dopingmittel zu quantifizieren und zu qualifizieren" (StA 3). Dies kann - jedoch offenbar nur in Einzelfällen - zu Verzögerungen führen.

„Wenn Gerichte darauf bestehen, dass jede einzelne Ampulle oder jedes einzelne Präparat auf den Wirkstoffgehalt überprüft wird, dann dauert es natürlich. Dann müssen nochmals Sachverständige und Wissenschaftler tätig werden. Das kann natürlich das Verfahren verlängern; aus meiner Sicht ist es nicht immer erforderlich, alle Ampullen zu untersuchen. Manche Gerichte sehen das anders." (StA 7)

\section{Bewertung}

a) Allgemeine Bewertung

Stimmen in der strafrechtswissenschaftlichen Literatur kritisieren die Verbotsnormen des $₫ 2$ ganz grundsätzlich. ${ }^{123} \mathrm{Da}$ sie keinen Bezug zum Wettkampfsport voraussetzten, werde auch eine sportliche Betätigung erfasst, die für die dopende Person eine reine Privatangelegenheit sei, bei der also keinerlei sportrechtliche Regeln eingehalten werden müssten. Sei aber der Einsatz von Dopingmitteln sportrechtlich unbedenklich, könne Doping die Integrität des Sports nicht beschädigen. Eine Rechtfertigung der Verbote durch das Ziel des Gesundheitsschutzes sei zwar möglich, doch würden die Vorschriften dann vor allem freiverantwortlich handelnde Men-

123 Zum Ganzen MüKo-StGB/Freund, 3. Aufl. 2018, AntiDopG \$S 1-4 Rn. 21. 
schen vor selbstschädigenden Handlungen schützen. Ein solcher strafrechtlicher Paternalismus sei kaum zu rechtfertigen. ${ }^{124}$

Dagegen ließe sich einwenden, dass eine Freiheit zum eigenverantwortlichen Konsum nicht zugleich ein Recht auf Weitergabe von Dopingmitteln impliziere. ${ }^{125}$ Diesbezüglich sind Gefahren etwa für jugendliche Sportler, die noch nicht freiverantwortlich über eine Selbstgefährdung entscheiden können, nicht auszuschließen. ${ }^{126}$ Zweifel an der Strafwürdigkeit bestehen dann jedoch weiterhin hinsichtlich der Implementierung und Umsetzung der Erwerbs- und Besitzverbote. Auch in den Experteninterviews äußerten die Befragten Bedenken gegen die Kriminalisierung von Erwerb und Besitz von Dopingmitteln. Ein Richter und ein Staatsanwalt stellten mit den Kritikern in der Literatur die Frage, ob der Schutz des Dopenden vor selbstschädigenden Handlungen prinzipiell eine Angelegenheit des Strafrechts sei:

„Ich denke, dass jeder selbstverantwortlich entscheiden muss, ob er Dopingmittel nimmt oder nicht." (StA 2)

„Also ist der Staat dazu da, jemand davon abzuhalten, sich mit aller Gewalt selbst zu schädigen, ja dann müsste man Alkohol auch verbieten. Also ein bisschen überspitzt jetzt. Da habe ich so ein gewisses Problem damit, weil...meine Güte. "( $R$ 2)

Der Gesetzgeber hat versucht, dem Legitimationsproblem der Erwerbsund Besitzstrafbarkeit dadurch zu entgehen, dass er sie mit der Gefahr einer Weitergabe der Dopingmittel an andere rechtfertigt; beide Tathandlungen stellen danach eine Vorfeldkriminalisierung der Abgabe, des Veräußerns oder Handeltreibens dar. Dieser Legitimationsansatz passt indes nicht zu dem Umstand, dass die Schwellenwerte für nicht geringe Mengen derart niedrig sind, dass die Verbote auch und vor allem reine Selbstnutzer erfassen (siehe oben 2.a.cc.).

Dass die Strafverfolgung vor allem Selbstnutzer aus dem Bereich des Bodybuildings betrifft, wird von einem Richter offen kritisiert. Dies führe le-

124 Näher dazu Freund, FS Rössner, 2015, S. 590; Lehner/Nolte/Putzke/Rössner, Anti-Doping-Gesetz, Vor $\$ 1$ Rn. 25.

125 Weber BtMG/ders., AntiDopG $₫ 1$ Rn. 6.

126 BT-Drs. 18/4898, 17; Eising, Die Strafbarkeit des Eigendopings, 2018, S. 86; ausführlich zur alten Rechtslage: Fiedler, Das Doping minderjähriger Sportler, 2013, S. 26 ff.; Heger, Strafrechtliche Besonderheiten im Umgang mit minderjährigen Leistungssportlern, in: Kauerhof/Nagel/Zebisch, 2010, S. 25 ff. 
diglich zu Verbesserungen der Strafverfolgungsstatistik, ohne aber am eigentlichen Problem des Sportdopings etwas zu ändern:

"Also es ist so, wie bei Drogenabhängigen, Heroin, Kokain, was weiß ich. Die können Sie jeden Tag durchsuchen, da werden Sie immer was finden. Das ist schön für die Statistik und da hat man was getan, bekämpft die Betäubungsmittelkriminalität. Wenn dann so ein Gesetz von oben kommt und wenn ich dann Zahlen brauche, dann gehe ich zu den Bodybuildern, misch die Szene auf und gut ist. Dann habe ich meine Zablen." (R2)

Im Gegensatz zu den leichten Ermittlungserfolgen gegen Selbstnutzer im Freizeitbereich und außerhalb des organsierten Sports stünden die fehlenden Erfolge im Kampf gegen das Doping im internationalen Spitzensport:

„Das andere ist halt, ja, man hat so den Eindruck, als ob das Problem da ist, aber ich meine, der Fisch stinkt vom Kopf. Wenn ich den Herrn Bach höre, dann krieg ich die Krise (...). Weil man hat nicht den Eindruck, dass das von oben runter wirklich ernst gemeint ist mit der Bekämpfung. " $(R$ 2)

\section{b) Gesetzgeberische Umsetzung}

Im Gegensatz zu der grundsätzlichen Kritik an der Anwendung des $₫ 2$ auf Selbstnutzer im Freizeitbereich stehen die überwiegend positiven Reaktionen auf die Ausgestaltung der Verbotstatbestände des $\$ 2$ im Übrigen. Hier ist nur eine dezidiert negative Stimme zu vernehmen gewesen, die ihr kritisches Urteil indes nicht näher begründete.

„Das ist ein schlechtes Gesetz, wenn ich nach meiner Einschätzung so sagen darf." ( $R$ 1)

Die deutliche Mehrheit der Befragten gelangt hingegen zu einem positiven Gesamtfazit, insbesondere wegen der in ihren Augen wichtigen Symbolik und der guten praktischen Handhabbarkeit der Vorschrift. So betonten zwei Staatsanwälte die besondere symbolische Bedeutung, die in der Ausgliederung der Verbotsnormen aus dem AMG und der Neuregelung im AntiDopG liege:

„Wenn es ein eigenes Gesetz dafür gibt, unterstreicht das natürlich dessen Wichtigkeit." (StA 2)

Ein anderer Staatsanwalt hob hervor, dass es wichtig gewesen sei, die Normen „aus diesem Wust AMG, wo alles Mögliche drin geregelt ist, rauszuneh- 
men. "Das AntiDopG habe den Normen „ein anderes Gewicht gegeben (...)." (StA 3)

Eine zweite Gruppe von Befragten betonte die leichte Handhabbarkeit des $\$ 2$ und gute Nachvollziehbarkeit seines Inhalts. Ein Richter meinte da$\mathrm{zu}:$

„Also man kann [die Vorschrift] umsetzen, (...) ohne viel Phantasie entwickeln zu müssen, wie das wobl gemeint sein könnte; das ist schon klar geregelt." ( $R$ 2)

Auch ein Staatsanwalt antwortete auf die Frage, ob $\mathbb{2}$ gelungen oder eher nicht gelungen sei:

"\$2 finde ich gelungen, weil er doch sehr übersichtlich ist. Ich habe da nichts auszusetzen, ich finde, das ist ein gut händelbares Gesetz." (StA 2)

Auch die Ermittler des Zolls begründeten ihr positives Fazit mit der Verständlichkeit und Praktikabilität der Vorschrift:

„Es ist leicht verständlich anwendbar für alle zu erklären. Nach einer gewissen Einarbeitung können die Kollegen insbesondere mit $\$ 2$ in Kombination mit den Verbotslisten, aber auch mit dem $\$ 2$ Abs. 3 mit der Stoffliste etwas anfangen. Was uns dann oftmals Probleme bereitet ist, welcher Stoff wohin kommt. Und weil sich die Stoffe schneller entwickeln, wie sag ich mal die Verbotsliste aktualisiert wird. Aber das können wir ganz gut, haben wir im Griff mit unserem Bildungs- und Wissenschaftszentrum, die uns da immer die neuesten Erkenntnisse liefern." (Zoll 1)

"Ja, die Arbeit hat sich durch die Einführung wesentlich erleichtert. Es ist ja ein sehr schmales, schlankes Gesetz, das uns endlich die Möglichkeit gegeben hat, einige Hilfsbrücken, die wir damals bauen mussten im Arzneimittelgesetz, die braucht man jetzt nicht mehr. (...) Jetzt hat man ganz klare Normen (...)." (Zoll 1)

Lediglich ein Ermittler sagte, dass das AntiDopG keine größeren Veränderungen gegenüber der Anwendung des AMG mit sich gebracht hätte. Seine Arbeit sei „eigentlich gleichgeblieben wie vorher auch.“ (Zoll 2) 
c) Verbesserungsvorschläge

aa) Materielles Recht

Materiell-rechtliche Verbesserungsvorschläge betrafen nicht die Tatbestände des $₫ 2$, sondern die Frage, ob (a) Mengen kumuliert werden dürfen, sowie (b) die Ausgestaltung der Dopingmittel-Mengenverordnung.

„Es gibt eine Klarstellung, die ich mir wünschen würde, und zwar (...) ist es so, dass selten nur ein Präparat konsumiert wird, sondern dass es immer eine Mischung ist, inklusive auch anderer Medikamente, die benutzt werden, um Nebenwirkungen zu bekämpfen, die dann entsprechend auch Dopingmittel sind. Es ist so, dass bei der Berechnung der nicht geringen Menge jeweils jeder Wirkstoff berechnet wird. Wenn ich jetzt z.B. drei verschiedene Präparate habe, und ich bin mit jedem Präparat einzeln beim 0,9-fachen vom Wirkstoff der nicht geringen Menge her, ist es so (...), dass die Werte kumuliert werden, so dass ich bei dreimal 0,9 eine 2,7-fache Überschreitung der nicht geringen Menge habe. Das wird aber nirgendwo klargelegt und nirgendwo dargestellt. (...) Es wäre schön, wenn man entweder im AntiDopG selber oder aber bei der Dopingmittel-Mengenverordnung oder in einem Begleitschreiben oder in der Gesetzesbegründung dazu deutlich machen würde, dass ausdrücklich kumuliert werden soll."(Zoll 3)

Dass die Möglichkeit der Kumulierung den Effekt verstärkt, Selbstnutzer im Fitnessbereich ohne Weitergabeabsicht zu kriminalisieren, wurde von dem Vorschlagenden nicht problematisiert. Darüber hinaus regte ein Interviewpartner eine Präzisierung der Dopingmittel-Verordnung an, die „manchmal nicht ganz eindeutig“ sei:

„Das weiß ich, weil unser Zolllabor findet und analysiert die Stoffe und die haben manchmal Fragezeichen im Kopf, wo es in welchen Teil oder ob überhaupt es in die Dopingmittel-Mengenverordnung passt aufgrund der Vielzahl der Stoffe, die gefunden werden. Also das klassische Testosteron ist überhaupt kein Ding, kein Problem, aber es gibt halt jede Menge neue FancyStoffe. Da wäre es schön, wenn das leichter oder einfacher wäre." (Zoll 3)

Zur Erläuterung dieser Problematik verwies der Interviewpartner auf eine konkrete Anfrage:

"Ich lese das einfach mal vor: ,Folgende Anregungen und Fragen zur Neuauflage der Dopingmittel-Mengenverordnung haben wir zusammengetragen: 1. Was genau sind, Andere mit anabol-androgenen Steroiden verwandte Stoffe? Gebören hier z.B. auch Trestolon, Epiandrosteron und Arimistan 
dazu? Wo fängt die Strukturverwandtschaft an und wo hört sie auf? Muss für die Stoffe ein wissenschaftlicher Nachweis vorliegen, dass sie anabol wirken? Wie müsste dieser Nachweis aussehen (Tierversuch, klinische Studie)? Sind hier auch die so genannten, Prohormone' gemeint?

2. Grundsätzlich wäre es ausgesprochen hilfreich, wenn die CAS-Nummern $z u$ den einzelnen genannten Stoffen angegeben wären. Denn nur hiermit lassen sich die genannten Namen (für einige gibt es zablreiche Synonyme) sicher einer chemischen Struktur zuordnen (Beispiel: Internetsuche nach Methylnortestosteron: Demnach könnte Metbylnortestosteron sein: $11 \beta-M e-$

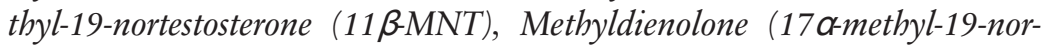
89-testosterone), Metribolone (methyltrienolone; R-1881; 17a-methyl-19nor- 89,11 -testosterone), Normethandrone (methylestrenolone; normethisterone; 17a-methyl-19-nortestosterone) oder Trestolone (7a-methyl-19-nortestosterone; MENT).

3. Es wäre hilfreich, wenn Selektive Androgen-Rezeptor-Modulatoren beispielhaft aufgezählt wären (LGD-4033, RAD 140, Enobosarm, Andarin, $Y K-11, \ldots$ )

4. Wie ist der Stoff SR9009 zu bewerten?

5. Bei Peptiden: Es gibt einzelne Peptide (z.B. GHRP6 und Pralmorelin), von denen es auch mit einer terminal angehängten Aminosäure (bisher: Glycin) modifizierte Varianten gibt. Sind diese auch unter den, Growth Hormone Releasing Peptides' miterfasst?" "(Zoll 3)

bb) Prozessrecht und Ermittlungsbefugnisse

In Bezug auf Ermittlungsmethoden wurden zwei Punkte angesprochen: die Einführung einer dem BtMG vergleichbaren Kronzeugenregelung sowie eine Erweiterung des Katalogs des $\$ 100$ a StPO um weitere Tathandlungsalternativen.

„Wichtig wäre für uns eine Einführung einer Kronzeugenregelung analog des $\$ 31$ BtMG. (...). Denn bis jetzt helfen wir uns ja nur aus mit einer nicht existierenden Kronzeugenregelung, die es ja im Antidopinggesetz nicht gibt, während man im BtMG ganz klar den 31er ziehen kann. Der wird auch regelmäßig bei uns bei größeren Aufgriffen dem Beschuldigten angeboten. Das heißt Klartext, ich habe jemanden mit einer großen Menge Rauschgift. Bevor ich ihn vernehme, muss er mir unterschreiben, dass ich ihm den \31 BtMG zur Kenntnis gegeben habe, damit er in dem Moment erkennen kann, wenn ich hier Ross und Reiter nenne und der Behörde bei der Aufklärung von Straftaten behilflich bin, dass ich mir dann Straferleichterung er- 
kaufen kann. Und das haben wir im AMG nicht, das wäre ein ganz klarer Vorteil, wenn wir sowas einführen können, analog dem $\$ 31$ BtMG. " (Zoll 1)

Auch eine Erweiterung des Katalogs des $\$ 100 a$ Abs. 2 StPO wurde von einem Staatsanwalt angeregt:

„Man könnte darüber nachdenken, was jetzt 100a anbelangt, ob man da vielleicht noch Erweiterungen, also dass man möglicherweise den kompletten $\$ 4$ Abs. 4 unter den 100 a stellt. Das wäre vielleicht eine sehr sinnvolle Überlegung. "(StA 1)

Mehrfach wurde von Seiten der Ermittler der Bedarf nach einer stärkeren Koordination und Abstimmung der Ermittlungen und einem Informationsaustausch angesprochen. Insbesondere in Bezug auf Lifestyle-Präparate, die nach kriminalistischer Erfahrung häufig von älteren Menschen oder Personen mittleren Alters nicht zum Zweck des Dopings im Sport verwendet werden, wünschte sich ein Ermittler eine bessere Vorstrukturierung der Verfahren und eine Reduzierung der aufwändigen und offenbar oft folgenlosen Ermittlungen:

„Wir haben Präparate, die sind zwar gelistet, aber bei gewissen Präparaten haben wir jetzt Erfahrungswerte, dass die evtl. nicht damit im Zusammenhang stehen, also überwiegend, und kann sich da austauschen. Man kann sich vielleicht mal einen anderen opus operandi speziell für so etwas überlegen, ob man das einfach in den Verfabrensablauf mit reinbringt, ob man da vielleicht überhaupt eine strafrechtliche Anbörung fübren müsste oder ob man sagt: Wir haben jetzt so viele Erkenntnisse aus dem Verfahren; bei gewissen Indikatoren, die wir hier vorliegen haben, könnte man evtl. das Verfahren noch ein bisschen abkürzen." (Zoll 2)

Von praktischer Bedeutung war ferner die Frage, wer für die sichergestellten Dopingmittel zuständig ist. Dazu bemerkte eine Interviewpartnerin, „dass es bei einigen Zollfahndungsämtern Probleme bzw. erhöhten Aufwand gibt und zwar dadurch, dass wir ja relativ viele Durchfubren sicherstellen, beschlagnahmen, Verfahren einleiten, ist es halt so, dass auch die Staatsanwaltschaften unterschiedlich agieren. Manchmal wird komplett eingestellt nach \$170 Abs. 2, manchmal auch nicht. Das Problem ist: Was passiert mit den Asservaten? Wer vernichtet die und auf wessen Kosten werden diese Asservate - für die Durchfuhr, wohlgemerkt - vernichtet? Viele Staatsanwaltschaften ziehen sich zurück und sagen: Ach nein, wir können das nicht, das soll nur der Zoll machen. Maximal wäre es für den Zoll selber möglich, das präventiv nach dem Zollfahndungsdienstgesetz sicherzustellen. Das ist aber auch ein enormer Aufwand für jedes 
einzelne Asservat. Da ist das Problem gerade, dass unterschiedlich verfahren wird, wer, wann, wie, was zerstört und wer welche Kosten trägt. Es sind halt relativ viele Mengen." (Zoll 3)

Schließlich verwies, auf Verbesserungen angesprochen, ein Vertreter des Zolls auf die zu geringen Ressourcen:

„Bei den Ressourcen ist es ganz klar der Personalkörper, der uns immer einen Riegel vorschiebt. Wir haben einen Personalfehlbestand, wie die komplette Bundeszollverwaltung. Und auch liegt zur Zeit der Schwerpunkt der Politik nicht im Bereich Zollfahndung, sondern Finanzkontrolle, Schwarzarbeit und FAU, so dass wir da ganz klar sagen müssen, da sind wir nicht over the top, sondern in der Priorität weiter hinten und konkret mit Auslandsbezug könnte ich nichts sagen, der Auslandsbezug mit den ausländischen Kollegen funktioniert wunderbar über diese Rechtshilfeschiene und bis jetzt waren wir immer in der Lage, schnellstmöglich die Kontakte herzustellen, um eventuell dann auch einfach nur abzufragen, hat man Interesse das Verfahren zu übernehmen, will man Ermittlungen fübren und das ist ja das, worauf es ankommt." (Zoll 1)

\section{III. Übergreifende Aspekte}

Im Folgenden werden Feststellungen der Evaluierungsstudie dargestellt, die beide der bislang untersuchten Vorschriften ( $\int \$$ 2-4) betreffen bzw. über die unmittelbar mit den materiell-rechtlichen Regelungen verbundenen Themen hinausgehen.

\section{Strafrahmen und Verjährung}

Die Strafrahmen für $₫ 2$ und $₫ 3$ werden in der gemeinsamen Vorschrift des $\mathbb{4}$ festgesetzt. Die Verjährung knüpft an das in $\$ 4$ normierte Höchstmaß an. Die mit $₫ 4$ verbundenen Fragestellungen sollen daher gemeinsam erörtert werden.

\section{a) Strafrahmen in $\mathbb{} 4$}

Verstöße gegen $₫ 2$ und $₫ 3$ werden grundsätzlich mit Freiheitsstrafe bis zu drei Jahren oder mit Geldstrafe geahndet ( $\mathbb{4}$ Abs. 1). Der Gesetzgeber hat sich insoweit für eine sanktionenrechtliche Parallelität entschieden, ob- 


\section{B. Ergebnisse der Evaluierung}

wohl das Schutzgut der das Selbstdoping betreffenden Straftatbestände nicht die Gesundheit ist, sondern die Integrität des organisierten Sports und seiner ethisch-moralischen Grundwerte wie Fairness und Chancengleichheit. ${ }^{127}$ Angesichts der Tatsache, dass das strafrechtliche Verbot des Selbstdopings besonders umstritten war, ist es kriminalpolitisch nachvollziehbar, dass der Gesetzgeber das Selbstdoping nicht stärker kriminalisiert als Verstöße gegen $\$ 2$.

Durchbrochen wird die sanktionsrechtliche Parallelität allerdings an zwei Stellen:

Zum einen beziehen sich die Qualifikationstatbestände des $\$ 4$ Abs. 4

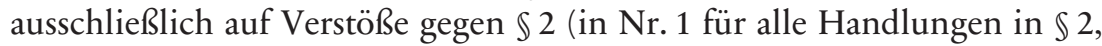
in Nr. 2 nur für $\$ 2$ Abs. 1 und 2). Hier gilt eine Strafandrohung von einem Jahr bis zu zehn Jahren Freiheitsstrafe; die Taten werden hierdurch zu Verbrechen hochgestuft. ${ }^{128}$ Relevant ist diese Vorschrift nicht nur für die Strafzumessung, sondern auch für die Zulässigkeit der Telekommunikationsüberwachung, die $\mathbb{1} 100 \mathrm{a}$ Abs. 2 Nr. 3 StPO auf Fälle des $\mathbb{} 4$ Abs. 4 Nr. 2 lit. b beschränkt, sowie für die Kronzeugenregelung des $\$ 46 \mathrm{~b} \mathrm{StGB}$, die nur für qualifizierte Straftaten gilt, nicht aber für den Grundtatbestand des $₫ 4$ Abs. 1 .

Zum anderen bestehen unterschiedliche Strafandrohungen für die Erwerbs- und Besitzstrafbarkeit. Der Erwerb oder Besitz von Dopingmitteln in nicht geringen Mengen nach $\mathbb{2}$ Abs. 3 wird mit einer Freiheitsstrafe von bis zu drei Jahren geahndet ( $\$ 4$ Abs. 1$)$. Wer hingegen gem. $\$ 3$ Abs. 4 ein Dopingmittel erwirbt oder besitzt, um es ohne medizinische Indikation bei sich anzuwenden oder anwenden zu lassen und um sich dadurch in einem Wettbewerb des organisierten Sports einen Vorteil zu verschaffen, dem droht eine Höchstfreiheitsstrafe von zwei Jahren ( $\$ 4$ Abs. 2).

Für die fahrlässige Begehungsweise (aller Tathandlungen nach $\$ 2$ Abs. 1) ist eine Freiheitsstrafe bis zu einem Jahr oder Geldstrafe vorgesehen, $\$ 4$ Abs. 6 .

127 BT-Drs. 18/4898, S. 18; Momsen, KriPoZ 2018, 21, 22; Graf/Jäger/Wittig/Eschelbach, Wirtschafts- und Steuerstrafrecht, 2. Aufl. 2017, $\$ 4$ AntiDopG Rn.1. Zum Begriff Kubiciel, KriPoZ 2018, 21 ff.

$128 \rrbracket 4$ Abs. 5 sieht allerdings die Möglichkeit eines minder schweren Falles vor; die Strafe nach Abs. 4 ermäßigt sich dann auf Freiheitsstrafe von drei Monaten bis zu fünf Jahren. 
aa) Anhebung der Höchststrafe in $\ 4$ Abs. 1

Einige der Experten aus der Justiz ${ }^{129}$ sprachen sich für eine Anhebung der Höchststrafe in $\$ 4$ Abs. 1 auf fünf Jahre aus. Dies wurde zum einen damit begründet, dass das bisherige Strafmaß den Anschein erwecke, dass es sich beim Umgang mit Dopingmitteln um Bagatellkriminalität handele.

„Ich finde den Strafrahmen bei der Höchststrafe viel zu niedrig. Ich würde absolut dafür plädieren, da einen Strafrahmen bis zur Freibeitsstrafe von fünf Jahren für das Grunddelikt aufzunehmen, weil ich meine, was ist das für ein Signal an die Öffentlichkeit, wenn ich sage Verstoß gegen Antidopinggesetzgebung ist wahnsinnig wichtig zu verfolgen und wir wollen hier sauberen Sport haben und sanktionieren es dann wie eine kleine Fundunterschlagung oder wie eine tätliche Beleidigung. ${ }^{130}$ Also vom Strafrahmen her weckt es so den Anschein von Bagatellkriminalität und das soll es ja nicht sein. Das heißt, wenn man ein Signal setzen will, dann sagen wir, die Verfolgung ist uns wichtig, dann müssten wir es zumindest auf eine Verfolgung wie für einen Ladendiebstahl upgraden. "(StA 3)

Die Experten wiesen zudem darauf hin, dass der unerlaubte Umgang mit Dopingmitteln mit der Betäubungsmittelkriminalität vergleichbar sei. Durch eine Anhebung der Höchststrafe könne man einen Gleichklang mit den Vorschriften des BtMG erreichen. ${ }^{131}$

"Jetzt hat man hier eben nur bis drei Jabre im Bereich Doping. Da spricht man eben doch eine mildere Strafe aus im Vergleich zu $\mathbb{S} 29$ Abs. 1 Betäubungsmittelgesetz. Also da sagt man, da sind es bis fünf Jabre. Da wäre sicherlich eine Erhöhung wünschenswert auffünf Jahre. "(StA 1)

Andere Interviewpartner aus der Justiz hielten die Strafrahmen hingegen für angemessen und konnten keinen Änderungsbedarf feststellen. Auch die Athletenvertreter lehnten eine Anhebung der Strafrahmen ab.

129 Drei der Staatsanwälte hielten die Anhebung für sinnvoll, vier Staatsanwälte sahen - ebenso wie die Richter - keinen Änderungsbedarf.

130 Die Höchststrafe für die tätliche Beleidigung beträgt allerdings zwei, nicht drei Jahre; $\mathbb{} 185$ StGB.

131 Angesprochen wurde von einem Experten auch, dass sich im AntiDopG keine Regelung zum Handeltreiben mit Waffen findet, das in $₫ 30$ a BtMG als Qualifikationstatbestand aufgeführt ist. 
„Das ist ja durchaus im Normalbereich. Ja. Also ich meine, man hat ja die volle Bandbreite. Von daher, es geht immer so weit nach oben, aber dann gibt man ja in den seltensten Fällen die Strafen. " $(R 2)$

"Also das ist ja so ein bisschen reduzierter Strafrahmen im Vergleich zu diesem Standardstrafrahmen sag ich mal, der oft ja dann Geldstrafe, Freiheitsstrafe vorgibt. Hier ist es ein bisschen reduziert. Ich halte es für ausreichend. Der Strafrahmen gibt genug Spielraum, um zu sachgerechten Entscheidungen im Einzelfall zu kommen." (StA 6)

"Ich bin nicht dafür, dass die Strafen noch erhöht werden. “ (A 2)

Dabei wurde auch darauf hingewiesen, dass eine Erhöhung des Strafrahmens für die Athleten keine abschreckende Wirkung entfalten würde. Entscheidender sei, die Strafverfolgung effektiver zu gestalten und ein reales Entdeckungsrisiko zu schaffen. ${ }^{132}$

„Ich glaube, das ist eine begrenzte Wirkung. Ich denke nicht, dass es jetzt dazu führt, dass man Doping verhindert. (...) Es war vorher schon so, dass man als Sportler sehr, sehr große Konsequenzen hat. Dass man aus dem Sport ausgeschlossen ist. Dass man erst einmal seinen Lebensunterhalt verliert. Dass man sozial geächtet wird. Das hat so weitreichende Folgen. (...) Man muss einfach das Risiko, erwischt zu werden, erhöhen und nicht die Strafe drakonisch erhöhen. "(A1)

bb) Inkonsistenz der geltenden Strafrahmen?

Einige Experten wiesen auf eine mögliche Inkonsistenz bei der Strafrahmenfestlegung für die Erwerbs- und Besitzstrafbarkeit hin. Wie oben dargelegt, ist der Erwerb oder Besitz von Dopingmitteln in nicht geringen Mengen nach $\ 2$ Abs. 3 mit einer Höchstfreiheitsstrafe von drei Jahren bedroht; für den Erwerb und Besitz von Mitteln zum Zweck des Selbstdopings gem. $\ 3$ Abs. 4 gilt hingegen eine Höchstfreiheitsstrafe von zwei Jahren. In der Diskrepanz der angedrohten Maximalstrafen wurde teilweise eine unnötige Privilegierung von Sportlern gesehen.

„[Es] ist tatsächlich nicht verständlich, warum der Spitzensportler nur mit einer Strafe aus einem Rahmen Geldstrafe bis Freiheitsstrafe bis zu zwei Jahren und der Breitensportler mit einem Strafrahmen und Geldstrafe bis Frei-

132 Dazu auch Eising, Die Strafbarkeit des Eigendopings, 2018, S. 165 ff. 
heitsstrafe von drei Jabren sanktioniert werden soll. Das hat so den Anschein einer Privilegierung und das ist nicht verständlich. (...) Da finde ich schon, das muss nicht sein. Zumal ja eigentlich der Leistungssportler auch von der Wertigkeit, finde ich, zumindest einem Breitensportler gleich verfolgt werden und eben nicht privilegiert werden sollte." (StA 3)

„Das ist ein Widerspruch: Dass der Besitz bei Spitzensportlern nicht so sehr bestraft wird wie bei Nicht-Spitzensportlern. Das ist so. Das finde ich nicht angemessen." (StA 2)

Andere Experten widersprachen der Kritik mit dem Argument, dass die Tatbestände unterschiedliche Voraussetzungen an die Strafbarkeit formulieren.

„Das ist ja eigentlich das Gegenteil einer Privilegierung. Spitzensportler sind strafbar, wenn sie auch geringe Mengen Dopingmittel besitzen, wohingegen ein Breitensportler, wenn er eine geringe Menge Dopingmittel besitzt, nicht strafbar ist." (StA 7)

\section{b) Verjährung}

Für Straftaten nach $\ 4$ Abs. 1 und 2 gilt die allgemeine Verjährungsfrist des $₫ 78$ Abs. 3 Nr. 4 StGB, die fünf Jahre beträgt. Erfüllt der Täter einen der Qualifikationstatbestände in $\$ 4$ Abs. 4, beläuft sich die Verjährungsfrist gem. $\$ 78$ Abs. 3 Nr. 3 StGB auf zehn Jahre. Hieran ändert auch das Vorliegen eines minder schweren Falles nach $\$ 4$ Abs. 5 nichts, vgl. $\$ 78$ Abs. 4 StGB. Die Fahrlässigkeitstaten in $\$ 4$ Abs. 6 verjähren gem. $\$ 78$ Abs. 3 Nr. 5 StGB in drei Jahren.

In der Aktenanalyse spielten Fragen der Verjährung keine Rolle. Dies dürfte auch dem Umstand geschuldet sein, dass das AntiDopG und mit ihm der Tatbestand des Selbstdopings erst im Dezember 2015, also vor weniger als fünf Jahren, in Kraft getreten ist.

Die Experten sahen hinsichtlich der Verjährungsfristen keinen Änderungsbedarf.

„Da gab es bis jetzt noch keine Probleme. Keine Änderungswünsche." (StA 4)

Die Erhöhung der Verjährungsfrist für die Qualifikationstatbestände des \4 Abs. 4 auf zehn Jahre gegenüber der Verjährung der Vorgängerregelung in $\$ 95$ AMG von fünf Jahren wurde von den Experten positiv bewer- 
tet. Hier kommt zum Tragen, dass die Vorschrift nicht mehr als Regelbeispiel, sondern als Verbrechenstatbestand ausgestaltet ist.

„Das spielt jetzt auch gerade im Bereich Aderlass eine Rolle, weil der Arzt ja 2011 in dem Bereich tätig war und jetzt eine ganze Reibe von Blutdopingbehandlungen wegfällt, weil zu Zeiten des AMG damals die Verjährungsregelung einfach 5 Jahre war. Mit 10jährigen Verjährungsfristen hätte man ja sehr viel weiter zurück gehen können. Also da sind jetzt alle Fälle, die vor den damaligen Olympischen Spielen in Sotschi im Februar 2014 waren, hinten runtergefallen. Das waren viele Fälle. Da waren also auch prominente Sportler dabei, die man hätte verfolgen können, wenn es damals die zehnjährige Verjäbrung schon gegeben hätte. "133

c) Bewertung

aa) Höchststrafe

Aus Sicht der Sachverständigen steht nicht zu befürchten, dass eine Höchststrafe von drei Jahren zu einer Relativierung des im AntiDopG normierten Unrechts führt. Eine Maximalstrafe von drei Jahren findet sich im StGB nicht nur in Fällen klassischer „Bagatellkriminalität“, sondern an ganz unterschiedlichen Stellen, etwa für die Datenhehlerei $(\$ 202 \mathrm{~d}$ StGB) und die Verbreitung jugend pornographischer ( $\$ 184 \mathrm{c}$ StGB) oder volksverhetzender Schriften ( $\mathbb{1 3 0}$ Abs. 2 StGB). Auch praktisch käme der Anhebung der Höchststrafe keine Bedeutung zu: Zum einen ist es an deutschen Gerichten üblich, sich - woraufhin der Experte R2 hinweist - an der Mindeststrafe, nicht an der Höchststrafe eines Delikts zu orientieren. Zum anderen hat die empirische Studie gezeigt, dass fast alle Verfahren wegen Selbstdoping-Delikten folgenlos eingestellt werden. Lediglich drei ausgewertete Verfahren endeten mit Strafbefehlen wegen Selbstdopings; zwei der darin ausgesprochenen Strafen sind am unteren Rand der nach $\$ 40$ Abs. 1 S. 1 StGB zulässigen Anzahl (30 bzw. 40 Tagessätze). Dass die geltenden Strafrahmen die Festsetzung einer der Tatschuld gerecht werdenden Strafe nicht zuließen, lässt sich folglich nicht feststellen. So gesehen, hat die Diskussion um die Erhöhung der Höchststrafen gegenwärtig keine

133 Das Kürzel des Staatsanwaltes wird hier nicht genannt, um durch die Bezugnahme auf das „Operation Aderlass“-Verfahren keine Rückschlüsse auf die Zuordnung anderer Äußerungen des Experten zuzulassen. 
rechtspraktische Bedeutung. Unabhängig davon ist auch zu bezweifeln, dass von einer Anhebung der Höchststrafe eine deutlich erhöhte generalund spezialpräventive Wirkung ausginge. ${ }^{134}$

Gleichwohl steht es dem Gesetzgeber selbstverständlich frei, aus symbolischen Gründen die Strafrahmen im AntiDopG an die des BtMG anzupassen. Zwingend ist eine solche Angleichung auch aus systematischen Gründen allerdings nicht; das AntiDopG und das BtMG ähneln einander zwar in ihrer Struktur und der Formulierung der Tatbestände, verfolgen jedoch unterschiedliche Schutzzwecke. Hinzu kommt, dass Betäubungsmittel erheblich größere Schäden beim Einzelnen und in der Gesellschaft verursachen als Dopingmittel.

\section{bb) Erwerbs- und Besitzstrafbarkeit}

Die Tatbestände in $\mathbb{2}$ Abs. 3 und $\$ 3$ Abs. 4 unterscheiden sich nicht allein mit Blick auf ihren Adressatenkreis, sondern in erster Linie hinsichtlich ihrer Schutzrichtung. $\$ 2$ Abs. 3 sanktioniert den Erwerb und Besitz von nicht geringen Mengen als Vorfelddelikt zum Handeltreiben. ${ }^{135} \$ 3$ Abs. 4 hingegen sanktioniert den Erwerb und Besitz von Dopingmitteln auch in geringen Mengen als Vorfelddelikt zum Selbstdoping. Da für das Handeltreiben und das Selbstdoping in $\$ 4$ Abs. 1 der gleiche Strafrahmen vorgesehen wird, ließe sich systematisch gut begründen, auch die Strafandrohungen für die jeweiligen Vorfeldtatbestände anzugleichen. In diesem Fall wäre es jedoch konsequent, die Höchststrafe von $\$ 2$ Abs. 3 abzusenken, um dem geringeren Unrecht eines Vorfeldtatbestandes Rechnung zu tragen. Ob dies rechtspolitisch ratsam ist, hat der Gesetzgeber zu entscheiden.

cc) Verjährungsregelungen

Nach Auffassung der Sachverständigen besteht derzeit kein Anlass, die Verjährungsregelungen zu ändern.

134 In der Kriminologie und Straftheorie ist anerkannt, dass Präventionseffekte weniger von der im Gesetz angedrohten Strafe und ihrer Höhe als vielmehr von der Wahrscheinlichkeit einer Entdeckung und Sanktionierung sowie von der Zügigkeit der staatlichen Reaktion auf die Tat ausgehen. S. dazu Eisenberg/ Kölbel, Kriminologie, 7. Aufl. 2017, $\$ 41$ Rn. 14, 22 ff., $\$ 42$ Rn. 5 ff.

135 BT-Drs. 18/4898, S. 25; Körner/Patzak/Volkmer/Volkmer, BtMG, 9. Aufl. 2019, AntiDopG $₫ 4$ Rn. 42. 
2. Einrichtung von Schwerpunktstaatsanwaltschaften und -gerichten

Schwerpunktstaatsanwaltschaften zur Bekämpfung der Doping-Kriminalität existieren derzeit in Freiburg, München und Zweibrücken; in anderen Bundesländern - etwa in Hessen - wird über ihre Einrichtung diskutiert. Ein Schwerpunktgericht für Dopingdelikte wurde bislang nur in Zweibrücken (Rheinland-Pfalz) eingesetzt.

a) Die Forderung von NADA und Athletenverbänden

Die NADA und die Athletenverbände haben in ihren Stellungnahmen die Schaffung weiterer Schwerpunkstaatsanwaltschaften und -gerichte gefordert. Begründet wird der Vorschlag mit der Komplexität der Materie, die eine besondere Spezialisierung insbesondere der Ermittlungsbeamten erfordere.

„Schließlich plädiert die NADA dafür, mehr Schwerpunktstaatsanwaltschaften für Verstöße gegen das Anti-Doping-Gesetz in Deutschland zu etablieren. Nur spezialisierte und geschulte Ermittler/-innen sind in der Lage schnell, effizient und zielgerichtet zu handeln. Die gute Zusammenarbeit zwischen der Schwerpunktstaatsanwaltschaft München I und den österreichischen Ermittlern/-innen ist als positives Beispiel anzuführen. "136

„Damit in Zukunft ähnliche Fälle aufgedeckt werden können, bedarf es ausreichender Kapazitäten und Expertise seitens der Ermittlungsbehörden. Wir halten es deshalb für sinnvoll, weitere Schwerpunktstaatsanwaltschaften zur Bekämpfung von Doping einzurichten." 137

In den Interviews wiederholten die Experten aus NADA und Verbänden ihren Wunsch nach einer stärkeren Spezialisierung in der Justiz. Die Experten berichteten dabei von schlechten Erfahrungen mit Staatsanwaltschaften und Gerichten, die über wenig Expertise im Umgang mit Dopingdelikten verfügten.

136 NADA, Öffentliche Anhörung des Sportausschusses des Deutschen Bundestages, Änderungs- und Ergänzungsbedarfe im Anti-Doping-Gesetz (AntiDopG), S. 5 .

137 Athleten Deutschland e.V., Anhörung des Sportausschusses, 23.10.2019, S. 3. 
"Wir haben zwar manchmal den einen oder anderen interessierten Staatsanwalt, der vielleicht auch schon mal einen Fall auf dem Tisch hatte, aber das hängt dann vom Zufall oder vom Glück ab, ob er gerade Zeit hat, ob er die Kapazitäten hat und diesen Fall dann auch betreiben kann." $(N)$

Die Befragten führten insbesondere auch die häufige Einstellung von Verfahren darauf zurück, dass die Staatsanwälte nicht mit dem AntiDopG und den praktischen Herausforderungen von Ermittlungen im Bereich des Sportdopings vertraut seien.

„Grundsätzlich hat man schon den Eindruck, und das kenne ich auch aus meiner vorherigen Verwendungsmöglichkeit, dass normale Staatsanwaltschaften einfach sehr weit weg von diesen sehr spezifischen Fragestellungen sind und die Fälle sehr, sehr schnell eingestellt werden - eigentlich schneller, als man schauen kann. Dass es da einfach einer gewissen Expertise bedarf im Bereich des Dopings und eines bestimmten Fachwissens, wie das funktioniert, wo man auch suchen muss. (...) Da haben wir schon das Gefübl gehabt, das kenne ich wirklich aus Erfahrung, dass Staatsanwaltschaften, die nicht mit diesen Fällen und Sachverhalten betraut sind - aus welchen Gründen auch immer, das möchte ich auch gar nicht bewerten - trotzdem die Fälle sehr schnell einstellen und da überhaupt nicht ermittelt wird." (A 3)

Mit den Schwerpunktstaatsanwaltschaften haben die Experten hingegen positive Erfahrungen gemacht.

„Bei den Schwerpunktstaatsanwaltschaften gerade in München, Freiburg und Zweibrücken, da sind die Organisationsformen so strukturiert, dass man zügig, professionell und zielgerichtet agieren kann. (...) Dann kann auch etwas wie jetzt in Erfurt, München und Seefeld entsprechend zügig umgesetzt werden. Da kann man dann sehen, was die Staatsanwaltschaft dann auch wirklich kann. (...). Das haben wir in wenigen anderen Bereichen, wo es keine Schwerpunktstaatsanwaltschaften gibt." $(N)$

b) Zur Einrichtung von Schwerpunktstaatsanwaltschaften

aa) Die Sicht der befragten Experten aus der Justiz

Die befragten Staatsanwälte sprachen sich überwiegend für die Einrichtung von Schwerpunktstaatsanwaltschaften aus. Sie teilten dabei die Einschätzung der Verbände, dass eine effektive Anwendung des AntiDopG 
spezielle Kenntnisse nicht nur des Rechts, sondern auch aus dem sportmedizinischen Bereich voraussetze.

"Also die Schaffung von Schwerpunkt-Staatsanwaltschaften halte ich für sehr, sehr sinnvoll. Es zeigt die Erfahrung immer wieder in Doping-Strafverfahren (...). Es ist durchaus ein Randbereich, der aber diverse Spezialkenntnisse erfordert, nur an den $\mathbb{S} 4$ Abs. 7 zu denken oder allein bei normalen Besitzfällen beim $\mathbb{S} 4$ Abs. $1 \mathrm{Nr} .3$ die Frage nicht geringe Menge, was ist eine nicht geringe Menge? (...) Vor dem Hintergrund halten wir eben Schwerpunktstaatsanwaltschaften für sehr, sehr wichtig und es wäre auch wünschenswert, dass eben neben Baden-Württemberg, Bayern, RheinlandPfalz auch die anderen Bundesländer den Schritt gehen würden, entsprechende Schwerpunktstaatsanwaltschaften zu gründen." (StA 1)

„Und ich finde schon, dass eine Spezialisierung erforderlich ist, weil da ja nicht nur Kenntnisse aus dem Bereich des Strafrechts von Nöten sind, sondern man braucht ja auch medizinische Kenntnisse, pharmazeutische Kenntnisse, anatomische Kenntnisse, chemische Kenntnisse. Also wie ist die Wirkung dieser Präparate im Körper? Wie werden sie hergestellt? Also all diese Geschichten kann man nicht leisten, wenn man Dopingsachen nebenbei in einem Betäubungsmittelreferat führt. Dafür sind diese Dopingfälle auch dann zu kompliziert und zu komplex, als dass man die einfach so nebenher bearbeitet. Also die Schwerpunktstaatsanwaltschaft halte ich für absolut notwendig und auch gut." (StA 3)

Auch Staatsanwälte, die selbst nicht in Schwerpunktstaatsanwaltschaften arbeiteten, hielten eine Konzentration der Ermittlungsarbeit für sinnvoll.

"Ja, das halte ich für sinnvoll, weil einfach das Know-how konzentriert wird und man dann doch vielleicht öfter einmal Leistungssportler einfach in größerem Umfang bekommt. Ich sehe das ja hier bei der StA. Wir haben wirklich selten Leistungssportler. Wenn man das im ganzen Bundesland konzentrieren könnte, hätte man sicher öfter damit Berührungspunkte. "(StA 2)

Neben besseren Kenntnissen der Materie wurde ein Vorteil der Schwerpunktstaatsanwaltschaften darin gesehen, dass die Staatsanwälte die bestehenden Dopingstrukturen in ihrem Bundesland besser überblicken.

„Vorteile der Schwerpunktstaatsanwaltschaft sind vor allem die Spezialisierung und auch die Möglichkeit, die jeweilige Dopingszene in dem jeweiligen Bundesland auch ordentlich anzugehen. Also das ist so eine Sache. Wenn es mal Leute gibt, die da hinfassen, dann werden die Fälle automatisch kom- 
men. Wir haben mit 170 Fällen im Jabr 2009 angefangen und haben im letzten Jabr 1500 Fälle gehabt." (StA 3)

Staatsanwälte in Schwerpunktstaatsanwaltschaften können zudem feste Kommunikationswege mit den Ansprechpartnern etwa beim Zoll oder der NADA aufbauen.

„Also Begründung auch für die Einrichtung der Staatsanwaltschaft war ja Dopingstrafrecht ist Sondergebiet und da sollen Sonderkenntnisse ausgebildet werden und dann möchte man auch eine bessere Vernetzung, so dass jetzt die zuständigen Staatsanwälte auch mit den entsprechenden Stellen bei Zoll, Polizei und NADA in Kontakt stehen. Das ist auch so. Also das ist mit Sicherheit ein Vorteil. Man hat feste Ansprechpartner. Und man kann so einen gewissen Automatismus einarbeiten. Was bei uns jetzt auch tatsächlich schon erfolgt ist, wenn man jetzt mehr mit den Verfahren befasst ist, ist man natürlich ein bisschen tiefer drin in der Materie, als wenn man jetzt als regulär zuständiger Staatsanwalt, keine Ahnung, fünf Fälle im Jahr in dem Gebiet bekommt. Dann ist man natürlich nicht so drin." (StA 6)

Andere Befragte hielten die Einrichtung von Schwerpunktstaatsanwaltschaften nicht für zwingend erforderlich. Ein Experte war der Ansicht, dass eine Spezialisierung an sich in jedem Deliktsbereich von Vorteil sei und das AntiDopG hier keine grundlegenden Besonderheiten aufweise.

„Ich meine, die Schwerpunktstaatsanwaltschaften bringen natürlich den Vorteil, dass man spezialisiertes Personal hat, die solche Verfabren dann einfacher und schneller lösen können. Natürlich ist es so, dass das für jeden Rechtsbereich gilt. Also spezialisierte BTMler oder spezialisierte Kollegen im Bereich Sexualstrafrecht oder ähnliches sind natürlich auch in ibrer Materie dann dementsprechend besser, ohne dass man die Notwendigkeit sieht, eine Schwerpunktstaatsanwaltschaft zu errichten. (...) Also ich halte es weder für verkehrt, noch bielte ich es für zwingend geboten." (StA 5)

Ein Staatsanwalt wies darauf hin, dass die Konzentration der Verfahren nach dem AntiDopG auch Schwierigkeiten bei der Verfahrensführung mit sich bringen könne; etwa dann, wenn neben dem Vorwurf des unzulässigen Umgangs mit Dopingmitteln auch Betäubungsmitteldelikte im Raum stünden.

„Es gibt aber halt auch Nachteile. Bei uns sind die in erster Linie dadurch begründet, dass oft Verfahren, die irgendwie zusammenhängen, dann so durch diese Zuständigkeitsverteilung so ein bisschen auseinandergerissen werden. Das heißt, in Koblenz, das ist also im Norden von Rheinland-Pfalz, 
wird beispielsweise bei einer Wohnungsdurchsuchung Dopingmittel und Betäubungsmittel festgestellt. Dann läuft das Betäubungsmittelverfahren mal erst in Koblenz, das Dopingmittelverfahren wird direkt an uns abgegeben, weil das bei der Polizei mittlerweile bekannt ist. Und dann läuft man sich so ein bisschen gegenseitig nach. Wer hat jetzt noch welches Verfahren und wer führt das jetzt sinnvollerweise zusammen und macht das überhaupt Sinn, das zusammenzuführen? Also da geht schon, bevor man die eigentliche Ermittlungsarbeit betreibt, geht schon viel Zeit ins Land bis man sich mal organisiert hat, wer jetzt diese Verfahren betreibt. Werden sie getrennt betrieben oder muss man sie zusammen betreiben, auch um etwaigen Straftageverbrauch oder so zu vermeiden. Das ist immer ein bisschen problematisch." (StA ${ }^{138)}$

Lediglich ein Experte (der selbst nicht in einer Schwerpunktstaatsanwaltschaft arbeitete) sprach sich gegen eine Konzentration der Zuständigkeit aus. Diese mache für herausgehobene Verfahren im Spitzensport Sinn, nicht aber für das Gros der Fälle im Bereich des Bodybuildings oder Breitensports.

„Die Schaffung von Schwerpunktstaatsanwaltschaften würde nur dann Sinn machen, wenn diese sich in der Tat auf Straftaten betreffend den Spitzensport konzentrieren würden. Spitzensport würde natürlich nicht nur die Sportler betreffen, sondern auch die Trainer, Betreuer und sonstiges medizinisches Umfeld von Spitzensportlern. Aber eine Konzentration von Verfabren nur bei einer StA, die sonstige Vergehen des Breitensports im Bodybuilding-Bereich zum Gegenstand hätte, würde einfach nur Ressourcen vergeuden, weil dann eine StA zuständig ist für einen Fall der aufgefundenen Dopingampulle im Rahmen einer Verkehrskontrolle in Nordhessen, während die Schwerpunktstaatsanwaltschaft in Südhessen sitzt. Das wäre einfach nur eine Verschwendung von Ressourcen." $\left(S t A^{139}\right)$

138 Die Nennung einer Nummer erfolgt hier aufgrund des konkreten Bezugs zum Bundesland nicht, um keine Rückschlüsse auf die Person des Staatsanwaltes bei anderen Antworten zu ermöglichen.

139 Die Nennung einer Nummer erfolgt hier aufgrund des konkreten Bezugs zum Bundesland nicht, um keine Rückschlüsse auf die Person des Staatsanwaltes bei anderen Antworten zu ermöglichen. 
bb) Erkenntnisse aus der Aktenauswertung

Die Aktenanalyse stützt - vorsichtig - die Einschätzung, dass Schwerpunktstaatsanwaltschaften durch etablierte Strukturen und größere Erfahrungen im Umgang mit Dopingdelikten eine effektivere Verfolgung leisten können. Allerdings dürfen die Erkenntnisse der Aktenauswertung hier nicht überbewertet werden, da sie sich auf Fälle des Selbstdopings beschränken.

Zwei der drei Strafbefehle wurden von der Schwerpunktstaatsanwaltschaft in Freiburg erlassen; von der dortigen Staatsanwaltschaft wurden auch die meisten Verfahren wegen Selbstdopings zur Verfügung gestellt. Hingegen lag aus der Schwerpunktstaatsanwaltschaft Zweibrücken nur ein einschlägiges Verfahren vor; das Verfahren wurde jedoch gründlich und unter Einsatz verschiedener Ermittlungsmaßnahmen durchgeführt. Bei allen Schwerpunktstaatsanwaltschaften zeigt sich, dass ein konkreter Tatvorwurf formuliert wurde, anstatt - wie sonst häufig beobachtet (siehe oben S. 12) - pauschal auf das AntiDopG zu verweisen. Auch die Kommunikation mit der NADA und dem Zoll verlief in den Schwerpunktstaatsanwaltschaften reibungslos. Die Schwerpunktstaatsanwaltschaften stellten auch seltener nach $\mathbb{1} 153$ StPO und häufiger nach $\$ 170$ Abs. 2 StPO ein. Dies kann als ein Indiz dafür gelten, dass die Tatbestandsmerkmale intensiver geprüft und bei auftretenden Ermittlungsschwierigkeiten nicht der einfache Weg einer Opportunitätseinstellung gewählt wurde.

\section{c) Zur Einrichtung von Schwerpunktgerichten}

Mit Blick auf die Einrichtung von Schwerpunktgerichten zeigte sich in den Interviews ein differenziertes Bild. Während der Vertreter der NADA Schwerpunktgerichte uneingeschränkt befürwortete, sprachen sich nur zwei der befragten Experten aus der Justiz eindeutig für eine zentrale gerichtliche Zuständigkeit aus. Die übrigen Experten sahen Vor- und Nachteile, die sich weitgehend die Waage hielten.

\section{„Schwerpunktgerichte ist sicherlich ein zweischneidiges Schwert." (StA 1)}

Erörtert wurde zunächst der Gewinn durch eine im Bundesland einheitliche Rechtsprechung bei der Auslegung des AntiDopG. Während teilweise eine konsistente Interpretation der Vorschriften für sinnvoll erachtet wurde, sahen andere die Gefahr einer Festigung der Rechtsprechung auf regionaler Ebene. 
„Es hat natürlich auch Nachteile, dass sich möglicherweise Rechtsprechung in gewissen Bahnen festigen kann, andererseits hat es natürlich auch den Vorteil, dass sich eine gefestigte Rechtsprechung schneller ergibt." (StA 4)

Ebenfalls eine Rolle spielten Praktikabilitätserwägungen. Einige der befragten Staatsanwälte sahen in der Einsetzung eines Schwerpunktgerichts die Möglichkeit, zeitaufwendige Reisen zu verschiedenen Gerichten im Bundesland zu vermeiden.

„Würde für uns den großen positiven Effekt haben, dass wir auswärtige Sitzungsdienste vermeiden könnten. Dann müssten wir nicht so viele Reisetätigkeiten unternehmen, die ja bei uns auch sehr viel Zeit in Anspruch nehmen, wo immer ein Kollege unterwegs ist." (StA 1)

Es wurde jedoch erkannt, dass eine zentrale Zuständigkeit des Gerichts im Umkehrschluss für den Beschuldigten oder die geladenen Zeugen erheblichen Aufwand bedeute.

„Für uns als Staatsanwälte ist es gut. Wir müssen nicht weit fahren, um das Ganze dann im Sitzungsdienst wahrzunehmen. Für die Beschuldigten, für sonstige Zeugen ist es nicht so gut. Die müssen dann alle nach Zweibrücken kommen. Es hat alles sein Für und Wider." (StA 5)

Auf diesen Aspekt wies auch ein Staatsanwalt aus Zweibrücken hin, der selbst Erfahrungen mit dem Schwerpunktgericht gemacht hatte.

"Man muss aber sagen, es hat natürlich auch immer den Nachteil: Wenn Sie das alles zusammenziehen, haben Sie ganz viele Zeugen, Polizeibeamte, der Beschuldigte selbst und die Rechtsanwälte. Die kommen oft von weit her. Wir fübren ja hier Verfahren aus ganz Rheinland-Pfalz, $d$. h. auch aus Mainz, aus Koblenz oder aus Frankental müssen dann alle immer hier anrücken. Während, wenn das jetzt so geregelt ist, dass die gerichtliche Zuständigkeit regulär bleibt, dass dann im Grunde genommen nur der Staatsanwalt fabren muss, um die Anklage da jetzt etwa zu vertreten. Hat wie so Vieles Vor- und Nachteile." (StA $\left.{ }^{140}\right)$

Die meisten Befragten waren jedoch der Ansicht, dass eine spezielle Zuständigkeit aus fachlicher Sicht sinnvoll sei.

140 Die Nennung einer Nummer erfolgt hier aufgrund des konkreten Bezugs zum Bundesland nicht, um keine Rückschlüsse auf die Person des Staatsanwaltes bei anderen Antworten zu ermöglichen. 
„Deswegen fordern wir Staatsanwaltschaften im Schwerpunkt, aber eben auch Gerichte, und das sehen wir auch verstärkt, dass ein Amtsgericht oder ein Landgericht Schwierigkeiten hat, im Alltag diese Spezialmaterie tatsächlich ordnungsgemäß darzustellen. Da hängt vieles an Aussage gegen Aussage, da hängt vieles davon an der Überzeugung der einzelnen Gutachter. Das ist Neuland für viele Gerichte, und auch da sollte man über eine besondere Schwerpunktsetzung nachdenken." $(N)$

Ein Staatsanwalt aus der Schwerpunktstaatsanwaltschaft in München berichtete, dass er und seine Kollegen die Gerichte im Umgang mit den weitgehend unbekannten Tatbeständen des AntiDopG häufig unterstützen müssten. Er schlug daher die Einrichtung von Schwerpunktgerichten zumindest in den jeweiligen OLG-Bezirken vor.

„Und Schwerpunktgerichte sind sinnvoll, weil wenn man sieht, wenn man bei einem kleinen bayerischen Amtsgericht anklagt und stellt halt fest, dass man auch unbedingt hinfahren muss, weil dann der Richter und auch der Verteidiger relativ nicht über so einen breiten Erfahrungs- und Kenntnisschatz verfügt. Es fängt schon mit den Gesetzen an, dass wir diese ja den Gerichten immer schon vorab mit Anklageerhebung zur Verfügung stellen, so dass sie da nicht groß nachgucken müssen. Die kriegen sie von uns mitgeschickt. Aber einfach auch, um bei der Sachverhaltsaufklärung vor Gericht $z u$ helfen. Also ist auch bei Gericht eine gewisse Spezialisierung wünschenswert. $\mathrm{Ob}$ das jetzt ein Gericht für ganz Bayern beispielsweise sein muss, oder $o b$ es nicht vielleicht auch besser wäre, dass in den drei OLG-Bezirken ein Schwerpunktgericht installiert werden könnte, würde ich zu letzterem tendieren, einfach um dann die lokalen Besonderheiten so ein bisschen berücksichtigen zu können, weil die Szene ja im OLG-Bezirk Bamberg sicher anders ist als im OLG-Bezirk München. Aber grundsätzlich ist auch da aus Gründen der Spezialisierung natürlich wünschenswert, dass da entsprechend auch Schwerpunktgerichte aufgestellt werden. " (StA $\left.{ }^{141}\right)$

Ein Staatsanwalt aus Zweibrücken lobte die Arbeit der Richter am Schwerpunktgericht und sah mit Blick auf die fachliche Expertise klare Vorteile einer konzentrierten Zuständigkeit.

"Also die Richter, die jetzt damit befasst sind, sind halt auch einfach ein bisschen tiefer drin in der Materie. Sie reißen nicht mehr erschrocken die Au-

141 Die Nennung einer Nummer erfolgt hier aufgrund des konkreten Bezugs zum Bundesland nicht, um keine Rückschlüsse auf die Person des Staatsanwaltes bei anderen Antworten zu ermöglichen. 
gen auf, wenn da mal eine Anklage kommt mit Doping. Das läuft hier wirklich ganz gut. Ich weiß, dass das bei den anderen Schwerpunktstaatsanwaltschaften beispielsweise nicht so ist. (...) Also den Vorteil, wenn man eine Konzentration haben will, das auf staatsanwaltschaftlicher und gerichtlicher Seite zu machen, würde ich mal sagen, ist aus rein fachlicher Sicht in der Tat gut." $\left(S t A^{142}\right)$

Die befragten Richter waren sich hier uneinig. Während sich einer der Richter aufgrund der Komplexität der Materie für die Einrichtung von Schwerpunktgerichten aussprach, hielt der andere die rechtlichen Schwierigkeiten für überschaubar und spezielle Zuständigkeiten bei Gericht nicht für erforderlich.

„Das mit den Schwerpunktgerichten ist sinnvoll, weil es sich um ein unübersichtliches Gesetz handelt und jemand, der nur gelegentlich damit zu tun hat, einige Zeit zubringen muss, um das zu durchdringen. " $(R 1)$

"Also ich denk nö, das ist nicht nötig. Das kriegt man hin. Die BtM-Verfahren sind ja auch nicht zentralisiert. Und das ist ja auch so eine Materie. (...) Von daher sehe ich das, also meine Güte, ja. Allein aus der Tatsache, dass es so selten vorkommt und man das Gesetz dann erstmal nachgucken müsste wabrscheinlich, wenn man als Wald-und Wiesenrichter das machen würde. Aber meine Güte, das gibt es ja öfter. Ich meine, ich habe jetzt hier einen Verstoß gegen das Tierschutzgesetz. Die gibt es ja auch nicht häufig. Dann guckt man halt dann auch mal rein. Also bitte." ( $R 2)$

\section{d) Bewertung}

Die strafrechtliche Verfolgung von Dopingverstößen stellt die Ermittlungsbehörden vor eine Vielzahl neuer Herausforderungen. Das AntiDopG gehört zum Nebenstrafrecht und regelt eine Materie, mit der Staatsanwälte weder in ihrer Ausbildung noch in der späteren Praxis regelmäßige Berührungspunkte haben. Die Anwendung des AntiDopG erfordert Kenntnisse über unterschiedliche Sportarten, deren Organisation, Wettbewerbe und Vergütungsstrukturen sowie von Einsatzbereichen und Wirkungsweisen leistungssteigernder Mittel, um den Nachweis illegaler Substanzen in Do-

142 Die Nennung einer Nummer erfolgt hier aufgrund des konkreten Bezugs zum Bundesland nicht, um keine Rückschlüsse auf die Person des Staatsanwaltes bei anderen Antworten zu ermöglichen. 
pingproben sowie die Aussagen von Sachverständigengutachten einordnen zu können. Die Aktenanalyse hat gezeigt, dass ein häufiger Grund für die Einstellung von Verfahren gerade die fehlende praktische Erfahrung mit Dopingfällen ist. Ermittler, die selten mit Dopingverfahren zu tun hatten, zeigten Unsicherheit etwa im Umgang mit einem Bestreiten des Beschuldigten und eine größere Zurückhaltung bei der Einholung von Sachverständigengutachten.

Zur Effektivierung der Strafverfolgung von Verstößen gegen das AntiDopG erscheint die Einsetzung von Schwerpunktstaatsanwaltschaften daher sinnvoll. Staatsanwälte, die speziell für den Bereich zuständig sind, können das notwendige rechtliche und sportmedizinische Wissen erwerben und feste Kontakte zu den externen Ansprechpartnern (NADA, Athletenverbände, Sachverständige) aufbauen. Der Vorteil einer zentralen $\mathrm{Zu}$ ständigkeit liegt nicht nur in der Konzentration von Kompetenz und Strukturen, sondern auch in der stärkeren Identifikation mit der Materie und der damit verbundenen Verantwortlichkeit für die Ahndung von Dopingstraftaten. Dass Verfahren aus "Scheu“ vor einem unbekannten Deliktsbereich oder allein aus Praktikabilitätserwägungen eingestellt werden, ${ }^{143}$ dürfte bei einer Schwerpunktstaatsanwaltschaft nicht - oder deutlich seltener - zu befürchten sein.

Um der berechtigten Kritik an einer Aufspaltung einheitlicher Sachverhalte in ein Verfahren nach dem AntiDopG und ein Verfahren etwa nach dem BtMG zu begegnen, wäre die Festlegung der Zuständigkeit der Schwerpunktstaatsanwaltschaften auch für verbundene Verfahren sinnvoll.

Mit Blick auf die Einsetzung von Schwerpunktgerichten hat sich in der Evaluierung ein ambivalentes Bild ergeben. Für Schwerpunktgerichte spricht - ebenso wie für Schwerpunktstaatsanwaltschaften - die besondere fachliche Expertise, die eine konzentrierte Zuständigkeit und die regelmäßige Befassung mit ähnlich gelagerten Sachverhalten mit sich bringt. Allerdings erscheint eine Spezialisierung der Gerichte weniger dringlich: Den Richter erreichen lediglich die wenigen zur Anklage gebrachten Verfah-

143 Hierzu StA 6: „Dann hat so eine Einstellung auch manchmal natürlich den Hintergrund, dass man sagt, ok, ich müsste hier noch weiter ermitteln und da noch weiter ermitteln und das ist alles irgendwie so ein bisschen kompliziert und ich weiß nicht so richtig. Und es lohnt sich meines Erachtens nicht, jetzt da noch tiefer einzusteigen, weil am Ende dann doch eine vergleichbar geringe Strafe nur zu erwarten ist. Das sind natürlich auch praktische Erwägungen. Also ich will jetzt nicht meinen Stand da in ein schlechtes Licht rücken, aber natürlich gibt es da auch immer einfach pragmatische Erwägungen. Lohnt sich das, da jetzt viel Arbeit zu investieren?" 
ren, so dass eine Einarbeitung - insbesondere auf Grundlage der staatsanwaltschaftlichen Vorarbeiten - problemlos möglich sein sollte. Ob ein Schwerpunktgericht eingesetzt werden soll, hängt daher maßgeblich von den Besonderheiten des jeweiligen Bundeslandes ab; eine klare Empfehlung sprechen die Sachverständigen deshalb hier nicht aus.

\section{Zusammenarbeit mit der NADA und $₫ 8$ AntiDopG}

Nach $₫ 8$ dürfen Gerichte und Staatsanwaltschaften der NADA personenbezogene Daten aus Strafverfahren von Amts wegen übermitteln, soweit dies aus Sicht der übermittelnden Stelle für disziplinarrechtliche Maßnahmen im Rahmen des Dopingkontrollsystems der NADA erforderlich ist und ein schutzwürdiges Interesse der betroffenen Person nicht entgegensteht. Mit der Regelung wollte der Gesetzgeber die NADA bei der Durchführung von Disziplinarverfahren unterstützen; die Ermittlungsergebnisse der Strafverfolgungsbehörden sollen der NADA bei der Sanktionierung von Sportlern und Athletenbetreuern zur Verfügung stehen. ${ }^{144}$

Nach Erkenntnissen dieser Studie ist die Zusammenarbeit zwischen der NADA und den Staatsanwaltschaften vor allem für die Strafverfolgungsbehörden von Bedeutung: In Fällen des Selbstdopings ist es häufig die NADA, die Strafanzeige erstattet oder über positive Dopingproben und andere Auffälligkeiten informiert. Weiterhin fungierte die NADA regelmäßig als Ansprechpartnerin für Fragen zur Art des Dopingmittels sowie der Testpoolzugehörigkeit eines beschuldigten Athleten.

"Also die NADA ist prinzipiell selbstverständlich immer Ansprechpartner. Also wenn es Verfahren gibt, wo beispielsweise die Einordnung etwa eines Sportlers als Testpoolathlet strittig sein könnte, wird natürlich die NADA kontaktiert."(StA 5)

„Ich finde den Austausch sehr wichtig, weil die NADA natürlich das entsprechende Know-how hat, auch bezüglich bestimmter Substanzen, wie die abgebaut werden o.ä., oder ob jetzt der Kandidat im Testpool ist. Das wird ja schon abgefragt." (StA 2)

Experten aus den Schwerpunktstaatsanwaltschaften, die regelmäßig an einem Erfahrungsaustausch mit Vertretern der NADA teilnahmen, berichteten von den Vorteilen bestehender auch informeller Kontakte.

144 BT-Drs. 18/4898, S. 35. 
"Ja, also man kennt die Leute einfach. Man kann da mal anrufen und kann auch so informell mal was fragen so vorab. Man hat E-Mail-Kontakt. Man trifft sich halt regelmäßig auf diesen Tagungen. Das ist immer sinnvoll, finde ich. Wenn man einen Ansprechpartner hat und wenn man die mal persönlich getroffen hat, dann ist ja die Hemmschwelle auch viel geringer, da mal anzurufen oder mal eine E-Mail hinzuschicken und mal zu fragen." (StA 6)

Während die NADA häufig die Staatsanwaltschaften mit wichtigen Informationen versorgt, ist der umgekehrte Informationsfluss insgesamt von geringerem Umfang und geringerer Bedeutung. Eine Kooperation im eigentlichen Sinne von $\mathbb{8} 8$ fand also eher selten statt. Einigen Staatsanwälten war die Norm nicht im Einzelnen bekannt.

„Da bin ich jetzt etwas überfragt. Ich schaue nochmal genau, was in $\$ 8$ drinstebt." (StA 7)

Eine etablierte Praxis, der NADA Ermittlungsergebnisse für deren Disziplinarverfahren zur Verfügung zu stellen, bestand bei den meisten Staatsanwaltschaften nicht. In Betracht der geringen Anzahl bislang einschlägiger Fälle ist dieser Befund allerdings nicht überzubewerten.

„Ich bin mir nicht so sicher, ob ich jetzt persönlich schon nach den Möglichkeiten des AntiDopG diese Übermittlungen durchgefübrt habe. Wenn es für das sportrechtliche Verfahren relevant und möglich ist, dann übermitteln wir natürlich die Erkenntnisse, soweit das rechtlich möglich ist. Aber ich persönlich kann mich jetzt nicht daran erinnern, das schon getan zu haben." (StA 4)

Wenn die Einleitung des Verfahrens auf einen Hinweis der NADA zurückging, wurde ihr - dies ergab sich auch aus den Akten - häufig die Abschlussverfügung übersandt.

„Die Informationsübermittlung besteht meistens darin, dass wir denen unsere Abschlussverfügung zur Kenntnis geben - das ist ja sozusagen diese Janusköpfigkeit des Sportrechts, dass das Antidopinggericht einerseits den strafrechtlichen justiziellen Arm hat und auf der anderen Seite den verbandsrechtlichen sportrechtlichen Arm, da sind die natülich auch auf die Informationen, wie wir am Ende das Verfahren bewerten, angewiesen und haben auch ein entsprechendes Recht, diese Informationen zu erhalten. "(StA 7)

Von einer intensiveren Kooperation berichteten hingegen zwei Staatsanwälte aus Schwerpunktstaatsanwaltschaften. Die Staatsanwälte forderten die NADA hier regelmäßig auf, den Athleten zunächst nicht über die posi- 
tive Dopingprobe zu informieren, um den Erfolg der Ermittlungsmaßnahmen - etwa einer Wohnungsdurchsuchung - nicht zu gefährden. Im Gegenzug wurden der NADA die Ermittlungsergebnisse mitgeteilt.

„In der Regel geht es ja in solchen Fällen los, die NADA erstattet die Anzeige. Und dann haben die in der Regel ja ein sportgerichtliches Verfahren parallel laufen. Und in der Regel würden wir dann der NADA aufgeben, mit der Benachrichtigung des Athleten noch zu warten, bis wir unsere strafprozessualen Maßnahmen durchgeführt haben. In der Regel wird es dann beim Fall Besitz und Erwerb oder anwenden lassen würde es dann auf eine Durchsuchung rauslaufen. Und dann würde die NADA natürlich auch im Rabmen der Untersuchung die Informationen sich versuchen zu verschaffen. Was hat die Untersuchung ergeben? Ist was gefunden worden? Wie hat sich der Atblet im Rabmen des Strafverfahrens zu der Geschichte geäußert, um das dann wiederum ggf. in ihrem sportgerichtlichen Verfahren zu verwerten. Also über diesen $\mathbb{} 8$ AntiDopG ist schon die Möglichkeit öfter im Rahmen eines Verfahrens und auch schon frübzeitig im Rabmen eines Verfahrens Akteneinsicht zu gewähren oder Auskunft zu erteilen schon wesentlich erleichtert worden. Sie teilen praktisch schon auch während des Laufs des Verfahrens Ermittlungsergebnisse mit und warten nicht bis zum Abschluss des Verfahrens, also nicht mit einer Abschlussverfügung, sondern können auch zwei, drei, vier Mal im Lauf eines Verfahrens dann Akteneinsicht gewähren oder Auskunft erteilen." (StA 3)

Auch wenn die Befragten selbst wenig oder keine Erfahrung mit $₫ 8$ gemacht hatten, hielten sie die Regelung für grundsätzlich sinnvoll. Änderungsvorschläge wurden nicht geäußert.

"Ich würde die Regelung für ausreichend erachten. Ich finde sie sehr sinnvoll, denn sie bietet einfach eine rechtliche Grundlage für diesen Austausch. Man hängt nicht so in der Luft mit diesem Austausch und muss sich überlegen, ja ist es jetzt zulässig oder nicht. Man hat jetzt wirklich eine rechtliche Grundlage, auch mit der man arbeiten kann und das begrüße ich sebr." (StA 1)

„Es ist auf jeden Fall ein großer Fortschritt zu dem Zustand davor, wo ja vieles einfach nicht so geregelt war. Die Position der NADA war ja nicht so klar geregelt. Ich sehe jetzt wenig Probleme oder hätte jetzt keine Wünsche, was sich da ändern könnte, damit das alles besser würde." (StA 2)

Diese positive Einschätzung teilten auch die Athletenvertreter.

„Grundsätzlich war ja immer das Problem, wie das mit dem Datenaustausch zwischen NADA, Gericht und Staatsanwaltschaft funktioniert. Da es 
an einer gesetzlichen Ermächtigungsgrundlage für den Datenaustausch fehlte, war das immer eine sehr einseitige Zusammenarbeit. Das war gar nicht böse oder kritisch gemeint. Natürlich hat die NADA immer geliefert, aber die Staatsanwaltschaften konnten umgekehrt diese Daten überhaupt nicht überliefern. Dass es das jetzt gibt in $\$ 8$, finden wir eben sehr gut, weil es einfach zeigt, dass beide Systeme zusammenarbeiten sollten und auch beide Systeme über unterschiedliche Kenntnisse verfügen und auch nochmals mit ibrer Fallexpertise gezieltes Wissen einfach weitergeben sollen, genau da, wo die Möglichkeiten einer Stiftung des bürgerlichen Rechts oder eines Sportverbandes enden, nämlich wirklich zu ermitteln und zu erforschen. Dann sich sozusagen nochmals auszutauschen und zu sagen: Wo könnte der andere belfen?" (A 3)

In ihrer Stellungnahme regt die NADA eine Erweiterung von $₫ 8$ auf sämtliche „zuständige, nationale und internationale Anti-Doping-Organisationen“ an. Die „internationale Vernetzung der NADA mit den Anti-DopingOrganisationen anderer Länder sowie mit der WADA“ mache es erforderlich, „den Informationsaustausch auch international zu erweitern“. Diese Forderung wurde im Experteninterview bekräftigt.

„Wir hatten einen konkreten Hinweis, aus Nordamerika kommend, dass ein Trainer mit Dopingsubstanzen nach Europa, insbes. nach Deutschland, einreisen möchte. Wir haben mit dem Zoll gemeinsam diese Information an den Zoll bringen können. Der Zoll konnte entsprechende Maßnahmen am Flughafen einsetzen und es fübrte auch dazu, dass sich bei dem Beschuldigten etwas finden ließ. Jetzt war es aber so: Er war kein deutscher Staatsangehöriger, auch wenn er sich in Deutschland nach dem Anti-Doping-Gesetz möglicherweise strafbar macht, ging es um die sportrechtliche Verfolgung und die NADA hatte da im Endeffekt auf gut Deutsch keine Eisen im Feuer, weil es - wie gesagt - keiner war, der in unsere Zuständigkeit fiel und die Möglichkeit, Akteneinsichtsnahmegesuche zu stellen, von einer ausländischen NADA sind dann eben nicht möglich. Da wäre dann an diesem Beispiel zu sehen und auch sicherlich am Beispiel der, Operation Aderlass', dass hier die Möglichkeit des internationalen Austausches gestärkt werden muss." (N)

\section{Schulungen}

In der Evaluierung ist auch danach gefragt worden, ob und durch wen Schulungen zum AntiDopG nach dessen Inkrafttreten erfolgt sind. Wäh- 
rend von Staatsanwälten und Richtern nicht von systematischen Schulungen berichtet wurde, scheint der Bereich des Zolls größere Schulungsaktivitäten entfaltet zu haben:

"Ja, wir haben Schulungen durchgeführt, das Zollkriminalamt insbesondere mit meinem Vorgänger, dem Kollegen B. Er hat ein Lehrgangskonzept konzipiert, so dass die Zollfahndung schon vor Einführung des Antidopinggesetzes einen Arzneimitteldopinglehrgang hatte, der alle damit bundesweit betroffenen Zollfahnder geschult hat, beim Zollkriminalamt mit diesem Deliktsbereich, was uns einen enormen Vorteil gegenüber der Polizei verschafft bat." (Zoll 1)

"Ja, teilgenommen habe ich, und zwar bei einem federführenden Kollegen. Man muss die regionalen Besonderheiten bei den Staatsanwaltschaften sehen, da sind dann auch manchmal regional unterschiedliche Schulungen. Der Kollege war schwerpunktmäßig bei der Zollfahndung tätig gewesen und ist mittlerweile in Pension. Der hat das dann ganz stark auf dem Fokus gehabt. Als das AntiDopG geschaffen wurde, habe ich mich mit ibm in Verbindung gesetzt und habe dafür Sorge getragen, dass er hier zum Flughafen kommt und hier eine Schulung hält. Ich habe dann entsprechend auch geschaut, dass wir so einen Pool für unseren Sachbereich bilden, uns unterhalten, wen wir daran teilnehmen lassen, wo das erforderlich wäre." (Zoll 2)

"Wir machen tatsächlich Schulungen für den Zollfahndungsdienst, also die Schulung entwerfen wir und dabei war dann selbstverständlich auch das AntiDopG enthalten. Also, wir machen Schulungen im Bereich Arzneimittel- und Dopingkriminalität." (Zoll 3)

Auch ein Vertreter des Bundeskriminalamts berichtete zwar nicht von Schulungen, aber von einem (nationalen und internationalen) Informations- und Erfahrungsaustausch:

"Wir übernehmen auch eine Politikberatung und wir nehmen sowohl an Gremien teil als auch, dass wir die z.T. ausrichten. Was ich damit meine, sind sog. Fachbearbeitungstagungen, wo aus dem ganzen Bundesgebiet die Fachdienststellen zusammengerufen werden oder sich einmal im Jahr treffen und ein Informationsaustausch stattfindet, als eben auch beispielsweise ein Informationsaustausch bei Veranstaltungen mit der NADA.“ (B)

Die Sportverbände selbst binden vor allem die NADA in die Schulungen ein. In welchem Ausmaß und mit welcher Nachhaltigkeit dies geschieht, ließ sich durch die Befragungen nicht feststellen. Immerhin berichtete 
aber eine Verbandsvertreterin davon, dass den Themen Integrität im Sport und Doping künftig noch größere Bedeutung zukommen soll:

"Wir wollen das Thema aber auf jeden Fall weiter vorantreiben. Es ist so, dass es ja die Antidoping-Beauftragten in unseren Mitgliedorganisationen gibt. Da haben wir früher die Schulungen durchgeführt bzw. die Antidoping-Beauftragten-Tagung macht im Moment die NADA in Absprache mit uns. Wir wollen da auch schauen, dass wir da wieder engere Beziehungen zu unseren Mitgliederorganisationen aufbauen und natürlich auch zur Beratung zur Verfügung stehen. “(D)

Gerade bei einem noch recht jungen Gesetz, das eine in rechtlicher und sportmedizinischer Hinsicht komplexe Materie regelt, sind Schulungen wichtig, um den Vorschriften zu praktischer Wirksamkeit zu verhelfen. Schulungen, wie sie der Zoll durchgeführt hat, sind daher ebenso sinnvoll wie Schulungen in den Verbänden und gegenüber Athletinnen und Athleten sowie ihrem Umfeld (Trainer, Betreuer und Ärzte). Dies nicht nur, um gelegentlich anzutreffende Informationsdefizite (etwa zur Normadressateneigenschaft) zu beseitigen, sondern vor allem, um auch auf diesem Weg einen kulturellen Wandel zu ermöglichen und die Geltung des Dopingverbots im individuellen und institutionellen Bewusstsein fest zu verankern. 


\section{Abschließende Stellungnahme der Sachverständigen}

\section{Allgemeine Bewertung}

Das strafbewehrte Verbot des Selbstdopings ( $\$ 3$ i.V.m $\mathbb{} 4$ Abs. 1 Nr. 4-5, Abs. 2) stellt die wesentliche materiell-rechtliche Neuerung des AntiDopG dar. Zudem hat der Gesetzgeber Straftatbestände des AMG in modifizierter und erweiterter Form in das AntiDopG überführt (vgl. $\mathbb{} 2$ i.V.m. $\mathbb{} 4$ Abs. 1 Nr. 1-3). Die Bekämpfung des Einsatzes von Dopingmitteln und Dopingmethoden im Sport dient laut $₫ 1$ dazu, die Gesundheit der Sportlerinnen und Sportler zu schützen, die Fairness und Chancengleichheit bei Sportwettbewerben zu sichern und damit zur Erhaltung der Integrität des Sports beizutragen. Diese Ziele werden in unterschiedlich starkem Ausmaß erreicht. Die Evaluation hat gezeigt, dass das AntiDopG in der Praxis vor allem die Gesundheit von Freizeitsportlern (vor selbstschädigenden Handlungen) schützt, weniger die Integrität des Sports.

\section{Selbstdoping $(\$ 3)$}

Verfahren wegen Selbstdopings im Bereich des Wettkampfsports machen einen vergleichsweise geringen Teil der Ermittlungsverfahren aus. Diese enden ganz überwiegend mit Einstellungen und nur in sehr seltenen Fällen mit einem Strafbefehl; aus den Verfahrensakten, die die Staatsanwaltschaften den Sachverständigen zur Einsichtnahme vorlegten, ging keine einzige Verurteilung hervor.

Dass nur wenige Ermittlungsverfahren Verstöße gegen das Selbstdopingverbot betreffen, liegt weniger an der tatbestandlichen Ausgestaltung der $\$ \mathbb{S 3}$, 4. Entscheidender ist, dass die Strafverfolgungsbehörden nur selten belastbare Informationen über Fälle des Selbstdopings erhalten. Den Sachverständigen lag kein Verfahren vor, das auf die Meldung eines Verbandes oder Sportvereins zurückging. In der Regel werden Strafverfahren nach Anzeigen der NADA bzw. Informationen der NADA über positive Dopingproben eingeleitet. Diese betreffen naturgemäß einzelne Sportler, nicht Strukturen und Netzwerke.

Zugleich bestehen auf Seiten der Ermittlungsbehörden teilweise noch Unsicherheiten im Umgang mit Fällen des Selbstdopings. Einige Staatsan- 
waltschaften stellten mit Blick auf die subjektive Tatseite deutlich überhöhte Anforderungen an die Annahme eines hinreichenden Tatverdachts. Auf die Einholung erforderlicher Sachverständigengutachten wurde häufig mit Hinweis auf die geringe Bedeutung der Sache verzichtet und die Verfahren nach $₫ 153$ StPO eingestellt.

Die Verfolgung von Selbstdoping könnte nach Ansicht der Sachverständigen durch Änderungen auf institutioneller Ebene und im Prozessrecht insbesondere durch die Schaffung weiterer Schwerpunktstaatsanwaltschaften sowie die Einführung einer Kronzeugenregelung - verbessert werden (siehe unten II., 2.). Damit Verstöße gegen das Selbstdopingverbot effektiv geahndet werden, kommt es jedoch maßgeblich auf den Willen der Akteure im Sport sowie die Durchsetzung regelmäßiger Dopingkontrollen an. Zudem halten die Sachverständigen eine moderate Überarbeitung der $\mathbb{S} 3$, 4 für notwendig, um den Anwendungsbereich des Selbstdopingverbotes klarer zu konturieren und bestehende Unklarheiten, insbesondere bei der Bestimmung des in $\$ 4$ Abs. 7 festgelegten Adressatenkreises, zu beseitigen (dazu unten II. 1.).

Insgesamt sollen die Empfehlungen eine Neufokussierung der praktischen Anwendung des AntiDopG ermöglichen: Ermittlungen wegen Verstößen gegen das Dopingverbot in Wettbewerben des organisierten Sports sollen stärker in das Zentrum treten - anstatt die Ressourcen von Zoll, Polizei und Staatsanwaltschaften weiterhin zu einem Gutteil für Ermittlungen gegen Bodybuilder und andere Selbstnutzer im Freizeitsport zu verwenden. Dazu dienen auch die Vorschläge, die $\$ 2$ betreffen.

2. Unerlaubter Umgang mit Dopingmitteln und unerlaubte Anwendung von Dopingmethoden $(\mathbb{2})$

Verstöße gegen die Verbotsnormen des $₫ 2$ bilden den Schwerpunkt der Ermittlungstätigkeiten im Bereich des AntiDopG. Dabei machen Selbstnutzer - vornehmlich aus dem Bereich Bodybuilding - den Großteil der Beschuldigten aus. Da diese nicht an Wettkämpfen teilnehmen, in denen Sport- und Wettkampfregeln zu beachten wären, können die Fairness und Chancengleichheit bzw. die Integrität des Sports durch die Einnahme von Dopingmitteln keinen Schaden nehmen. Geschützt wird damit in erster Linie der dopende Freizeitsportler vor selbstschädigenden Handlungen. Dazu trägt maßgeblich der Umstand bei, dass die Dopingmittel-Mengenverordnung nach Auskunft der befragten Experten vergleichsweise niedrige Schwellenwerte für die Annahme einer nicht geringen Menge festsetzt, 
so dass auch Bodybuilder für den Erwerb und Besitz von Dopingmitteln strafverfolgt werden, die sie selbst nutzen und mit denen sie keinen Handel treiben. Ein großer Teil der Ermittlungsressourcen, die das AntiDopG in Anspruch nimmt, wird mithin auf die Verfolgung von Freizeitsportlern verwendet, die ohne Wettkampfbezug dopen und vor allem sich selbst schädigen.

Ermittlungen gegen Personen, die mit Dopingmitteln im größeren Umfang handeln oder diese herstellen, bilden hingegen die Ausnahme. Wie weit die Rechtspraxis gegenwärtig den Verbotstatbestand des $\$ 2$ versteht, zeigt eindrücklich ein Ermittlungsverfahren, das gegen einen übergewichtigen Beschuldigten geführt worden ist, der ein Diätpräparat bestellt hatte, das einen vom AntiDopG erfassten Inhaltsstoff enthält.

„Ein offensichtlich übergewichtiger Mann hat Dopingmittel bestellt, jedenfalls (...) in der Dopingmittel-Mengen-Verordnung aufgeführte Produkte. Er hat angegeben bei der Polizei, er habe ein Mittel zur Gewichtsreduktion gesucht. Allein das setzt ja noch nicht voraus, dass der Tatbestand entfällt, weil auch das Training zur Gewichtsreduktion, was mit Dopingmitteln unterstützt wird, ist Doping im Sport. Etwas komplizierter wird die Sache dann, wenn er sagt, er wollte nie Sport machen, sondern das Präparat als Gewichtsreduktionsmittel beschreibt." (StA 5)

Die Strafbarkeit des Verhaltens hängt bei einem solch weiten Verständnis des Tatbestandes folglich davon ab, ob der Beschuldigte die Gewichtsreduktion (auch) durch sportliche Betätigung erreichen will oder allein durch die Einnahme des Präparates. Treibt er Sport, könnte er in den Anwendungsbereich des $\$ 2$ geraten; verzichtet er auf eine sportliche Betätigung und nimmt lediglich das Präparat ein, handelt er klar straflos. Mit den Zwecken des Gesetzes - Gesundheitsschutz, Schutz der Integrität des Sports - lässt sich die unterschiedliche Behandlung der Fallvarianten nicht rechtfertigen. Mehr noch: Die Diskrepanz bestätigt die Zweifel daran, dass die weite Auslegung des Erwerbs- und Besitzverbotes mit den Zielen des AntiDopG und dem Zweck des $\$ 2$ Abs. 3 vereinbar ist. Um die Tätigkeit der Ermittlungsressourcen besser auf strafwürdige Fälle auszurichten, wird unten (dazu unten II.2.b.) ein Vorschlag unterbreitet, der den in $\mathbb{1} 1$ genannten Zielen Rechnung trägt und das von $\$ 2$ Abs. 3 verfolgte gesetzgeberische Anliegen konsequenter umsetzt; eine Änderung von $\mathbb{} 2 \mathrm{Abs} .3$ oder gar eine Neukonzipierung des Legitimationsmodells des $₫ 2$ ist dafür nicht erforderlich. 


\section{Konkrete Empfehlungen der Sachverständigen}

1. Materiell-rechtliche Änderungen

a) Selbstdoping

Strafbar sind Verstöße gegen das Selbstdoping-Verbot nur für einen kleinen Kreis von Sportlerinnen und Sportler: Testpool-Athleten und diejenigen, die aus der sportlichen Betätigung unmittelbar oder mittelbar Einnahmen von erheblichem Umfang erzielen ( $\$ 4$ Abs. 7). Der Gesetzgeber begründet diese Einschränkungen des Anwendungsbereiches mit teleologischen sowie kriminalpolitischen Gründen.

In teleologischer Hinsicht wird argumentiert, dass die „Integrität des organisierten Sports (...) insbesondere durch das Verhalten der in der Öffentlichkeit wahrgenommenen Leistungssportlerinnen und Leistungssportler bedroht“ werde. An einer anderen Stelle heißt es sogar, dass „zu einem Vertrauensverlust in das Sportsystem (...) Manipulationen durch Doping nur bei den herausgehobenen Sportlerinnen und Sportlern führe." ${ }^{145}$ "Unrecht, das auch strafwürdig ist,“" werde „in diesem Rahmen lediglich von den Sportlerinnen und Sportlern verwirklicht, die ihren Sport leistungsund wettkampforientiert auf hohem Niveau betreiben bzw. erhebliche Einnahmen aus der sportlichen Tätigkeit ziehen." Nur diese Sportlerinnen und Sportler stünden für den organisierten Sport, nur sie stellten Vorbilder dar und nähmen das Vertrauen in Anspruch, ihre sportlichen Erfolge mit lauteren Mitteln erlangt zu haben.

Diese Auffassung überzeugt nicht. Denn an dem von Verbänden organisierten und regelgeleiteten Wettbewerbssport nehmen ganz überwiegend Sportlerinnen und Sportler teil, die keine Testpool-Athleten sind und keine erheblichen Einnahmen erzielen. Anders gewendet: Der organisierte Sport, dessen Integrität das AntiDopG schützt $(\$ 1)$, findet ganz überwiegend in dem Bereich statt, den $₫ 4$ Abs. 7 von der Strafbarkeit ausnimmt. Teleologisch betrachtet, ist die Strafbarkeitseinschränkung des $\$ 4$ Abs. 7 StGB also gerade nicht folgerichtig, sondern eine begründungsbedürftige Abweichung von dem durch $\$ 1$ vorgezeichneten Zielpfad. Der Gesetzgeber stützt diese Abweichung auf das (sozialpsychologische) Argument, dass (nur oder vor allem) Spitzensportler bzw. Sportler, die mit ihrem Sport Einnahmen von nicht unerheblichem Umfang erzielen, Vorbilder darstellten und Vertrauen in integres Verhalten in Anspruch nähmen. Dieser Be-

145 Sämtliche Zitate BT-Drs. 18/4898, S. 31. 
trachtung liegt der Typus eines Spitzensportlers im Bereich von Sportarten mit hoher Medienöffentlichkeit zugrunde (Formel-Eins-Fahrer, Profiboxer, Bundesliga-Fußballspieler). ${ }^{146}$ Deren Involvierung in einen DopingSkandal wird ein besonders großes Potenzial zugeschrieben, das Vertrauen in die Integrität des Sports zu schädigen. Das mag so sein, auch wenn die erste Verurteilung des Profiboxers Felix Sturm wegen Selbstdopings nur kurzzeitig Gegenstand der Medienberichterstattung gewesen ist und kaum für öffentliche Empörung gesorgt hat. Darauf kommt es aber auch nicht an. Denn für den Schutz der Integrität des Sports ist nicht erheblich, ob „die Öffentlichkeit" von der staatlichen Reaktion auf Doping erfährt. Entscheidend ist vielmehr, dass die gesellschaftlichen Gruppen, die es angeht, Kenntnis davon haben, dass der Staat auf ein integritätsverletzendes Doping reagiert. Die relevante Personengruppe sind also vor allem Sportlerinnen und Sportler; besonders relevant jene Sportlerinnen und Sportler desselben Sports und desselben Wettbewerbes: Sie sind es, für die die Regeleinhaltung Bedeutung hat; sie füllen den Begriff der Integrität des Sports mit Leben. Und sie sind es auch, die - in der Regel als einzige - vom Doping eines Konkurrenten Kenntnis erlangen. Ob also ein Sportler im Kampf um die Fußball-Meisterschaft der Bezirksliga oder der Dritten Liga dopt, ist sub specie Integritätsschutz nicht relevant; erst recht spielt es keine Rolle, ob und wieviel der Spieler mit seinem Sport verdient.

Insbesondere die Einschränkung auf Sportlerinnen und Sportler, die Einnahmen von erheblichem Umfang erzielen, begegnet mit Blick auf den gesetzlichen Schutzzweck grundlegenden Bedenken. Die Strafwürdigkeit einer Einnahme von Dopingmitteln kann nicht davon abhängen, ob der Sportler oder die Sportlerin durch die Teilnahme an Wettbewerben Geld verdient. Derartige finanzielle Aspekte sind dem Schutzgut der Integrität des Sports fremd. Die Erwägung, dass erst die Aussicht auf Einnahmen Sportlerinnen und Sportler zum Doping motivieren könne, entspricht nicht den Realitäten des Sports in Deutschland. Für den weit überwiegenden Anteil der Sportlerinnen und Sportlern stellen finanzielle Vorteile nicht die maßgebliche Motivation für ihre Entscheidung, Leistungssport $\mathrm{zu}$ betreiben ${ }^{147}$ und auch nicht für die Anwendung von Dopingmitteln

146 Vgl. zu diesen Beispielen BT-Drs. 18/4898, S. 32.

147 Siehe etwa Gröpel/Schoene/Wegner, Implizite und explizite Motive von Leistungsund Freizeitsporttreibenden, Zeitschrift für Sportpsychologie (2015), 22, S.6. Dass sich in den wenigsten Sportarten für Athleten in Deutschland überhaupt erhebliche Einnahmen erzielen lassen, ergibt sich auch aus der Studie des Bundesinstituts für Sportwissenschaft, Breuer/Wicker, Sportökonomische Analyse der Lebenssituation von Spitzensportlern in Deutschland, 2010. 
dar. ${ }^{148}$ Zudem stellt das Selbstdoping im Sinne des $₫ 4$ strafwürdiges Unrecht dar; besondere subjektive Absichten (namentlich: Erzielung von erheblichen Einnahmen) sind folglich nicht konstitutiv für das Unrecht, sondern könnten allenfalls Gegenstand eines Qualifikationstatbestandes sein. Im Hinblick auf den Grundtatbestand ist eine entsprechende zusätzliche Strafvoraussetzung aber teleologisch sachfremd.

Für eine Einschränkung des Anwendungsbereichs der $\$ 4$ Abs. 1 Nr. 4 und 5 bzw. Abs. 2 kann daher nicht der Zweck des Gesetzes sprechen, sondern allenfalls die Sorge um einen unverhältnismäßig weitreichenden Einsatz des Strafrechts auch gegen „reine Freizeitsportler“. Die Voraussetzung der (geplanten) Teilnahme an einem „Wettbewerb des organisierten Sports" stellt allerdings eine ausreichend hohe Hürde da, um den reinen Freizeitsport von einer Strafbarkeit auszunehmen. Nach der Gesetzesbegründung werden hierdurch ,in erster Linie Sportwettbewerbe des Spitzen- und Leistungssports" erfasst, an denen Freizeitsportler gerade nicht teilnehmen. Private Turniere werden durch das Tatbestandsmerkmal folglich vom Anwendungsbereich des Verbots ausgeschlossen. ${ }^{149}$ Dass Freizeitsportler an Wettbewerben des organisierten Sports teilnehmen, ist eine klare Ausnahme, die sich weitgehend auf größere Laufveranstaltungen beschränkt. Doping dürfte bei solchen Personengruppen auch keine praktische Bedeutung haben. ${ }^{150}$ Auch mit Blick auf den Amateursport ${ }^{151}$ - der nach dem Schutzzweck des Gesetzes zu Recht normativ erfasst wird - erscheint das Risiko einer Überkriminalisierung im Lichte der durch die Evaluierung festgestellten sehr zurückhaltenden Anwendungspraxis als äußerst gering. Hinzu tritt, dass bei entsprechenden Veranstaltungen keine Dopingkontrollen stattfinden, die den Staatsanwaltschaften Hinweise auf Doping geben könnten (wie die Evaluierung gezeigt hat, sind insbesondere Hinweise der NADA für die Entstehung eines Anfangsverdachts entscheidend). Es ist daher nicht damit zu rechnen, dass ohne die Existenz der Strafbarkeitseinschränkung des $\mathbb{4}$ Abs. 7 gegen eine große Anzahl von Freizeit- und Amateursportler wegen des Verdachts des Selbstdopings strafrechtlich ermittelt würde.

148 Nach Einschätzung der NADA ist ein finanzieller Vorteil nur einer von mehreren Gründen für Doping: https://www.nada.at/de/praevention/dopipedia/marke tshow-warum-wird-gedopt.

149 BT-Drs. 18/4898, 27.

150 Sollten die Ermittlungsbehörden tatsächlich in einem solchen Fall Kenntnis von Selbstdoping erlangen, kann - auch im Lichte der aktuellen Praxis - davon ausgegangen werden, dass ein solches Verfahren eingestellt wird.

151 BT-Drs. 18/4898, 31. 
Hingegen hat sich im Rahmen der Evaluation die Einschränkung des Täterkreises in $\$ 4$ Abs. 7 als das zentrale materiell-rechtliche Problem bei der Strafverfolgung von aufgedeckten und auch strafwürdigen Fällen des Selbstdopingverbots erwiesen. Insbesondere die Bestimmung der „erheblichen Einnahmen" bereitet in der Praxis Schwierigkeiten und ist nicht selten ein wesentlicher Grund für die Einstellung des Verfahrens. Aus diesem Grund hat das AntiDopG bislang wenig Bedeutung für den Schutz der Integrität des Sports durch das strafbewehrte Verbot des Selbstdopings erlangt.

Diesem Befund lässt sich auf zwei Wegen begegnen: (1) Durch eine Konkretisierung von $\$ 4$ Abs. 7 oder (2) durch eine Streichung der Einschränkung.

(1) Die verbreitete Unsicherheit darüber, was eine erhebliche Einnahme ist und was nicht, ist zwar für ein neues Gesetz nicht untypisch. Allerdings dürfte es angesichts der Seltenheit strafrechtlicher Verurteilungen einige Zeit dauern, bis diese Fragen höchstrichterliche Klärung erfahren. Da Athletinnen und Athleten offenbar von einem deutlich höheren Schwellenwert ausgehen als die Staatsanwälte und sich daher der tatsächlichen Strafbarkeitsrisiken nicht bewusst sind, erscheint - wenn man eine entsprechende Eingrenzung des Täterkreises beibehalten will - eine Präzisierung der Norm sinnvoll. Wie hoch der Gesetzgeber die Einnahmen ansetzen möchte, ist eine rechtspolitische Frage. Im Einklang mit der bisherigen Praxis würde es sich anbieten, Einnahmen von erheblichem Umfang dann anzunehmen, wenn sie nach Abzug der für die Ausübung des Sports getätigten Ausgaben die in $\$ 8$ Abs. 1 SGB IV genannten Grenzen der Geringfügigkeit überschreiten.

Im Rahmen einer Reform des $₫ 4$ Abs. 7 wäre zudem zu empfehlen, die Regelung zum Täterkreis in $\$ 3$ zu überführen. Die Festlegung einer Tätereigenschaft stellt eine erhebliche Strafbarkeitseinschränkung dar, die auch aus Gründen der Verständlichkeit - bereits in der Verbotsnorm selbst und nicht erst in der Sanktionsvorschrift enthalten sein sollte.

Ob die Anforderungen an die Täterqualität als Tatbestandsmerkmal oder als objektive Bedingung der Strafbarkeit gestaltet werden, liegt im gesetzgeberischen Ermessen; allerdings besteht wenig Anlass, den besonderen Weg einer objektiven Strafbarkeitsbedingung zu wählen.

(2) Eine moderate Reform von $\$ 4$ Abs.7 nach den oben skizzierten Grundsätzen vermag allerdings die grundlegenden Probleme, die mit der Begrenzung des Adressatenkreises verbunden sind, nicht zu lösen. Eine Konkretisierung der „erheblichen Einnahmen“ schafft zwar Klarheit bezüglich des Maßstabes. Sie ändert jedoch nichts daran, dass die Ermittlun- 
gen aufwendig sind: Die Staatsanwaltschaften müssten die Einnahmen des Beschuldigten durch den Sport und seine Ausgaben für den Sport rekonstruieren. Dadurch werden Opportunitätseinstellungen bereits in einem frühen Stadium begünstigt.

Die Beschränkung des Täterkreises wurde vom Gesetzgeber eingeführt, um „reine Freizeitsportler“ von einer Strafbarkeit auszunehmen. Wie oben dargelegt, zieht $₫ 4$ Abs. 7 den Kreis der Adressaten allerdings deutlich enger (siehe ausführlich oben B.I.2.c.). Von einer Strafbarkeit ausgeschlossen sind auch Leistungssportler, die (noch) nicht in einen Testpool aufgenommen wurden (etwa Athleten aus dem B-Kader) und die - was in einer Vielzahl von Sportarten üblich ist - keine erheblichen Einnahmen generieren. Der Sinn und Zweck des AntiDopG (insbesondere des $\$ 3$ ), die Grundwerte des Sports vor einer Beeinträchtigung durch Doping zu schützen, wird jedoch unabhängig davon berührt, ob der Teilnehmer in einem sportlichen Wettbewerb ein Testpool-Athlet ist oder etwa kurz davorsteht, diesen Status zu erreichen. ${ }^{152}$ Maßgeblich für das zu schützende Rechtsgut der „Integrität des Sports“ ist die Teilnahme an einem Wettbewerb des organisierten Sports, in dem sportspezifische Regeln zu beachten sind. Nicht maßgeblich ist hingegen, ob die Athletinnen und Athleten einem Testpool angehören oder ob sie Einnahmen aus diesen Wettbewerben erzielen. ${ }^{153}$ Auch für die öffentliche Wahrnehmung dürfte es allein auf die Sportveranstaltung selbst ankommen; welcher der Teilnehmer konkret betroffen ist, wird für die Beeinträchtigung des Vertrauens in die Fairness im Sport nicht von Bedeutung sein.

Hinzu tritt, dass die Beschränkung des Täterkreises grundlegenden Gerechtigkeitserwägungen widerspricht und ehrliche Sportlerinnen und Sportler nur unzureichend schützt. Die Regelung in $\$ 4 \mathrm{Abs} .7$ führt zu einer unterschiedlichen Behandlung von Sportlerinnen und Sportlern, die in demselben Wettbewerb gegeneinander antreten. Einem Testpool-Athleten dürfte es kaum vermittelbar sein, dass er dem AntiDopG unterfällt, ein Konkurrent, der auf derselben Veranstaltung ebenfalls um den Sieg kämpft, hingegen nicht.

152 So auch MüKo-StGB/Freund, 3. Aufl. 2018, AntiDopG $\$ \mathbb{S 1 - 4}$ Rn.112; Brill, SpuRt 2015, $153 \mathrm{ff}$.

153 Insbesondere ist es mit Blick auf das Schutzgut nicht überzeugend, dass die Erzielung erheblicher Einnahmen für die Strafbarkeit entscheidend sein soll: Für die Frage, ob die Integrität des Sportes verletzt wird, können monetäre Aspekte keine Rolle spielen. 
Mit Blick auf den gesetzlichen Schutzzweck ist jede Teilnahme an einem Wettbewerb des organisierten Sports unter Anwendung eines Dopingmittels, das dem Sportler oder der Sportlerin einen Vorteil verschaffen soll, strafwürdig.

Die Sachverständigen empfehlen daher, die Einschränkung des Täterkreises in $₫ 4$ Abs. 7 ersatzlos zu streichen.

Es erscheint ratsam, in der Begründung das strafbarkeitseinschränkende Potential des Tatbestandsmerkmals des „Wettbewerbs des organisierten Sports" herauszustellen. Aktenauswertung und Experteninterviews deuten darauf hin, dass dem Merkmal bislang (zu) wenig Bedeutung beigemessen wird. In diesem Zusammenhang könnte auch darauf hingewiesen werden, dass die Teilnahme an Wettbewerben des Bodybuildings nicht von $\mathbb{S} \$ 3,4$ erfasst wird. Zwar stellt der Trainingsprozess eine sportliche Tätigkeit dar, der spätere Wettbewerb ist jedoch nicht sportlicher Natur, sondern eine Präsentation der Körpergestaltung. ${ }^{154}$

Sollte der Gesetzgeber an einer ausdrücklichen Einschränkung des Normadressatenkreises festhalten wollen, wäre es ratsam, ein Abgrenzungskriterium zu wählen, das den intendierten Anwendungsbereich zielgenau umschreibt. In Betracht kommt hier die Formulierung eines Strafbarkeitsausschlusses für den „Freizeitsportler“. Die erforderliche Legaldefinition könnte dabei insbesondere in Zusammenarbeit mit der NADA entwickelt werden, die derzeit an einer entsprechenden Definition für den nationalen Anti-Doping Code arbeitet. Zu beachten wäre dabei, dass die Voraussetzungen praktisch gut handhabbar sein müssen und keinen größeren Ermittlungsaufwand zur Folge haben dürfen. Um die Probleme der geltenden Gesetzeslage zu vermeiden, sollten in die Definition insbesondere keine finanziellen Aspekte einbezogen werden.

b) Unerlaubter Umgang mit Dopingmitteln $(\$ 2)$

aa) Um die knappen Ressourcen der Staatsanwaltschaften auf Fälle von größerer Bedeutung konzentrieren zu können und zugleich dopende Freizeitsportler nicht mit grundrechtsintensiven Ermittlungsverfahren zu überziehen, bieten sich zwei Möglichkeiten an:

154 Hierzu auch Brill, SpuRt 2015, 153, 154; MüKo-StGB/Freund, 3. Aufl. 2018, AntiDopG $\$ \$ 1$ 1-4 Rn. 39. 
(1) Zunächst könnte man mit einer Stimme in der Literatur einen Wettkampfbezug für die Anwendbarkeit der Verbotsnormen des $\$ 2$ verlangen. ${ }^{155}$ Damit rückten die Verbote des $₫ 2$ stärker in die Nähe von $₫ 3$ und ließen sich nicht nur mit dem Ziel des Gesundheitsschutzes rechtfertigen, sondern auch mit dem Schutz der Integrität des Sports. Eine solche Interpretation hätte jedoch zur Folge, dass der vom Gesetzgeber als Primärzweck des $\$ 2$ ausgewiesene Gesundheitsschutz in den Hintergrund tritt. Auch wenn man den Wettkampfbezug allein auf $\$ 2$ Abs. 3 beschränkte, blieben dann der Erwerb und Besitz großer Mengen an Dopingmitteln straflos, die erkennbar nicht mehr dem Eigengebrauch dienen können. Damit würde die gesetzgeberische Entscheidung, Erwerb und Besitz als Vorstufen für ein Handeltreiben unter Strafe zu stellen, dogmatisch und praktisch unterlaufen.

(2) Vorzugswürdig ist es daher, auf einem anderen Weg zu einer dem gesetzgeberischen Ziel angemesseneren Auslegung des $₫ 2$ Abs. 3 und zu einem zielgenaueren Einsatz von Ermittlungsressourcen zu gelangen. Der Gesetzesbegründung zufolge stellen der von $\$ 2$ Abs. 3 erfasste Erwerb und Besitz nicht geringer Mengen von Dopingmitteln „erfahrungsgemäß Vorstufen für einen Handel mit den entsprechenden Mitteln dar."156 Weil diese Mittel bei Anwendung zu Dopingzwecken die Gesundheit von Sportlerinnen und Sportlern in besonderem Maße beeinträchtigen, solle „,bereits die Gefahr der Weitergabe effektiv verhindert werden. Deshalb werden bereits Handlungen, die typischerweise nur der Vorbereitung für die Weitergabe dienen, verboten." 157

Indes hat die Evaluierung gezeigt, dass Verfahren auch gegen Beschuldigte geführt werden, bei denen die aufgefundenen Mengen so gering sind, dass der Erwerb bzw. Besitz - auch nach Einschätzung der Ermittler - nicht als Vorstufe für einen Handel bzw. eine Weitergabe an Dritte anzusehen ist. In den Interviews bestätigten die Befragten, dass sich Verfahren vor allem gegen „Selbstnutzer“ richteten. Als wesentlichen Grund für diesen Befund nannten sie dabei insbesondere die Dopingmittel-Mengen-Verordnung, deren Mengen so niedrigschwellig seien, dass sie bei fast jedem Fund erreicht werden und folglich auch Personen ohne Weitergabe- oder gar Handelsabsicht erfassen.

155 MüKo-StGB/Freund, 3. Aufl. 2018, AntiDopG, $\$ \$ \$ 1-4$ Rn. 23, 64.

156 BT-Drs. 18/4898, S. 25; MüKo-StGB/Freund, 3. Aufl. 2018, AntiDopG, $\mathbb{S} \$ 1-4$ Rn. 58.

157 Dazu BT-Drs. 126/15, S. 24 f.; BT-Drs. 18/4898, S. 25. 
Erschwerend kommt hinzu, dass in der Rechtspraxis oftmals mehrere für sich genommen geringe Mengen unterschiedlicher Stoffe kumuliert werden, um durch die Addition die Überschreitung des Schwellenwerts zu einer nicht geringen Menge zu erreichen. Diese Praxis ist vor dem Hintergrund des vom Gesetzgeber verfolgten Ziels und des von ihm gewählten Legitimationsmodells nicht unbedenklich. Werden etwa zwei angebrochene Ampullen mit unterschiedlichen Stoffen aufgefunden, so ist die Weitergabe der Reste zum Zweck des Handeltreibens nahezu undenkbar; Verfahren wurden in diesen Fällen gleichwohl geführt.

Nicht zuletzt hat die Evaluierung gezeigt, dass sich Ermittlungen zunehmend gegen Personen richten, die sog. Anti-Aging und Lifestyle-Produkte zur Selbstnutzung ohne Dopingabsicht erwerben. Auch dies ist ein Beleg dafür, dass die Grenze zur straflosen Selbstnutzung geringer Mengen derzeit nicht sinnvoll gezogen wird.

Die Sachverständigen empfehlen daher, die Dopingmittel-Mengen-Verordnung stärker an das vom Gesetzgeber mit $\mathbb{2}$ Abs. 3 verfolgte Ziel anzupassen und Mengenwerte festzuschreiben, bei deren Erreichung eine Absicht des Handeltreibens einigermaßen sicher vermutet werden kann.

Werden die strafbarkeitsbegründenden Mengen signifikant angehoben, so ist ein Verbot der Kumulierung von nicht geringen Mengen nicht erforderlich. Sollte dies nicht umgesetzt werden, empfehlen die Sachverständigen (hilfsweise, da diese Maßnahme nicht ausreichen würde, um die faktische Kriminalisierung von Eigenkonsum zu verhindern), in der Dopingmittel-Mengen-Verordnung klarzustellen, dass zumindest keine Kumulierung von nicht geringen Mengen erfolgen darf .

bb) Die Fahrlässigkeitstatbestände des $₫ 4$ Abs. 6 haben in der Praxis keine Bedeutung erlangt. Dies mag auch daran liegen, dass eine fahrlässige Herstellung, ein fahrlässiger Handel oder ein fahrlässiger Besitz von Dopingmitteln „zum Zwecke des Dopings beim Menschen“ konstruktiv kaum vorstellbar ist. In der Praxis sind Verfahren, bei denen eine fahrlässige Begehung von den Ermittlern in Betracht gezogen wird, sehr selten und enden in aller Regel mit einer Einstellung. Dies zeigt auch, dass Staatsanwaltschaften das Unrecht und die Schuld - so vorhanden - als gering erachten. Der vom Gesetzgeber genannte Charakter der Vorschriften als „Gesundheitsgefährdungsdelikte“ zwingt nicht zu einer Kriminalisierung. Vielmehr wäre zu begründen, weshalb die Gesellschaft vor einer fahrlässigen Verletzung der Verbote des $₫ 4$ Abs. 1 Nr. 1-3 geschützt werden muss. Diese Begründung ist kaum zu führen. Die Evaluierung hat gezeigt, dass es ei- 
nes solchen Schutzes tatsächlich nicht bedarf. Denn anders als die von der Gesetzesbegründung erwähnten Vorbildtatbestände (fahrlässige Körperverletzung, fahrlässige Brandstiftung und fahrlässige Trunkenheit im Verkehr) spielt $\$ 4$ Abs. 6 in der Praxis keine Rolle. Der Ermittlungsaufwand und die mit den Ermittlungen einhergehenden grundrechtsrelevanten Belastungen der Beschuldigten stehen mithin in keinem angemessenen Verhältnis zum Ertrag und vor allem nicht zur verwirkten Schuld.

Da für die Fahrlässigkeitstatbestände in $₫ 4$ Abs. 6 kein zwingender Strafgrund existiert, die Kombination aus Fahrlässigkeitselementen und besonderen Absichten konstruktiv problematisch ist und es an einem praktischen Bedürfnis für einen „Auffangtatbestand“ fehlt, sprechen die Sachverständigen die folgende Empfehlung aus:

\section{Die Sachverständigen empfehlen, $₫ 4$ Abs. 6 ersatzlos zu streichen.}

\section{Allgemeine prozessuale und institutionelle Änderungen}

a) Kronzeugenregelung und Hinweisgebersysteme

Die relativ geringe praktische Bedeutung des Verbots des Selbstdopings in der Strafverfahrenspraxis und die vergleichsweise wenigen Verfahren gegen Spitzensportler erklären erfahrene Ermittler damit, dass „wir die Fälle nicht bekommen" (StA 3). Dies liegt auch daran, dass sich Doping in diesem Bereich im Verborgenen vollzieht; weder treten die Folgen der Taten sichtbar zu Tage noch existieren geschädigte Dritte, die von den Taten unmittelbar erfahren und diese anzeigen könnten. Die Ermittlungsbehörden sind also in besonderer Weise auf Informationen von Sportlerinnen und Sportlern und ihrem Umfeld angewiesen. Diese haben aber wenig Anreiz, Wissen von Straftaten zu offenbaren - insbesondere dann, wenn sie damit strafrechtliche Ermittlungen auf sich ziehen könnten. Zwar ist es den Staatsanwaltschaften auch ohne die Existenz einer Kronzeugenregelung möglich, eine Aufdeckungshilfe durch die Einstellung des Verfahrens zu honorieren. Für die Sportlerinnen und Sportler dürfte jedoch die Aussicht auf eine solche, gesetzlich nicht normierte Praxis nicht die notwendige Sicherheit bieten. Erst mit der Einführung einer Kronzeugenregelung bringt der Gesetzgeber verbindlich zum Ausdruck, dass und auf welche Weise er die Preisgabe relevanter Informationen privilegieren will. Es läge daher im Interesse einer effektiven Verfolgung von Dopingdelikten, einen sichtbaren Anreiz für eine Aufklärungshilfe durch Sportlerinnen und Sportler in Form einer klaren gesetzlichen Regelung zu schaffen. Dazu kann auch die 
Schaffung einer Kronzeugenregelung gehören, wenn sich die Schaffung einer solchen in das legislative Gesamtgefüge einpassen lässt.

Aus diesem Grund empfehlen die Sachverständigen zum einen, dass die Sportverbände ihre Athletinnen und Athleten stärker als bislang über die Existenz und Funktionsweise der Hinweisgebersysteme der NADA und WADA informieren.

Zum anderen empfehlen die Sachverständigen zu prüfen, ob sich eine auf die Besonderheiten des Dopings im Sport zugeschnittene Kronzeugenregelung in das AntiDopG einführen lässt.

b) Schwerpunktstaatsanwaltschaften und Schwerpunktgerichte

Die Evaluierung hat deutlich gemacht, dass die Ermittlung von Verstößen gegen das AntiDopG eine Reihe von rechtlichen, medizinischen und kriminalistischen Spezialkenntnissen voraussetzt. Diese sind, wie die Evaluierung offenbart hat, vor allem bei Schwerpunktstaatsanwaltschaften vorhanden, während sich bei anderen Staatsanwaltschaften ohne entsprechende Spezialisierung häufig Probleme bei der Anwendung des AntiDopG zeigten.

Aus diesem Grund empfehlen die Sachverständigen den Bundesländern, dem Vorbild der Länder Baden-Württemberg, Bayern und Rheinland-Pfalz zu folgen und Schwerpunktstaatsanwaltschaften einzurichten.

Eine vergleichbare Notwendigkeit für die Einrichtung von Schwerpunktgerichten hat die Evaluierung hingegen nicht ergeben. 


\section{Annex: Leitfaden Staatsanwälte}

A. Kerndaten

1) Wie lange arbeiten Sie in einer Abteilung, die mit Doping zu tun hat?

2) Mit wie vielen Fällen des Dopings hatten Sie in etwa zu tun?

\section{B. Phänomen / Sachverhalte}

\begin{tabular}{|c|c|c|}
\hline \multicolumn{3}{|c|}{ Wie sehen typische Fälle von Doping aus, mit denen Sie zu tun haben? } \\
\hline Was möchten wir & Weitere Fragen & Notizen \\
\hline & $\begin{array}{l}\text { - Welche Sportarten / -verbände } \\
\text { sind überwiegend betroffen? } \\
\text { - Welche Dopingmittel / -metho- } \\
\text { den werden überwiegend ver- } \\
\text { wendet? } \\
\text { - Wie wurde das Doping aufge- } \\
\text { deckt? Haben Sie dafür ein illus- } \\
\text { tratives Bsp.? } \\
\text { - Was steht im Fokus: Hintermän- } \\
\text { ner oder Selbstnutzer? Breiten- } \\
\text { sport oder Leistungssport? } \\
\text { - Geht es auch um Fälle organi- } \\
\text { sierter Kriminalität? Eher Hin- } \\
\text { termännerbereich oder eher La- } \\
\text { bore? Haben Sie dafür ein illustra- } \\
\text { tives Bsp.? }\end{array}$ & \\
\hline
\end{tabular}




\section{Ermittlungen}

\section{Teil: Anfangsverdacht}

Woraus speist sich idR Ihr Anfangsverdacht

- in Bezug auf den unerlaubten Umgang mit Dopingmitteln bzw. unerlaubte Anwendung von Dopingmethoden ( $(2)$ ?

- in Bezug auf das Selbstdoping $(\$ 3)$ ?

\begin{tabular}{|c|c|c|}
\hline $\begin{array}{l}\text { Was möchten wir } \\
\text { wissen? }\end{array}$ & Weitere Fragen & Notizen \\
\hline $\begin{array}{l}\text { (von Amts wegen, An- } \\
\text { zeige durch Hinweisge- } \\
\text { ber, Anzeige durch } \\
\text { Sportverband bzw. An- } \\
\text { zeige durch NADA) }\end{array}$ & & \\
\hline Andere Verfahren & $\begin{array}{l}\text { - Kommt es häufig vor, dass sich } \\
\text { aus einem anderen Verfahren } \\
\text { (zB gegen Hintergrundlabore) } \\
\text { Ansätze für weitere Verfahren er- } \\
\text { geben? Können Sie ein illustrati- } \\
\text { ves Beispiel nennen? } \\
\text { - } \quad \text { Kommt es häufig vor, dass sich } \\
\text { aus Verfahren, die wegen Verstö- } \\
\text { ßen nach } ₫ 2 \text { geführt werden, Er- } \\
\text { mittlungsansätze für Verfahren } \\
\text { wegen Selbstdopings nach } ₫ 3 \text { er- } \\
\text { geben? }\end{array}$ & \\
\hline NADA & 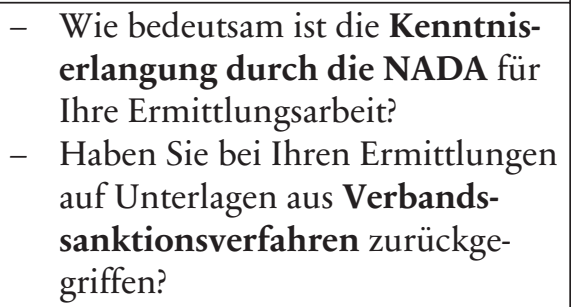 & \\
\hline $\begin{array}{l}\text { Bewertung und } \\
\text { Empfehlungen }\end{array}$ & $\begin{array}{l}\text { - Halten Sie die bestehenden Wege } \\
\text { der Kenntniserlangung für ausrei- } \\
\text { chend? Ggf.: Was könnte getan } \\
\text { werden, um die Kenntniserlan- } \\
\text { gung der StA zu verbessern? }\end{array}$ & \\
\hline
\end{tabular}




\begin{tabular}{|c|c|c|}
\hline \multicolumn{3}{|c|}{$\begin{array}{l}\text { Teil 2: Ermittlungs- und Zwangsmaßnahmen / Nachweisprobleme } \\
\text { Welche Ermittlungs- und Zwangsmaßnahmen setzen Sie ein und mit } \\
\text { welchem Erfolg? (Unterschiede bei } ₫ 2 \text { und } ₫ 3 \text { ?) }\end{array}$} \\
\hline $\begin{array}{l}\text { Was möchten wir } \\
\text { wissen? }\end{array}$ & Weitere Fragen & Notizen \\
\hline $\begin{array}{l}\text { Angewandte } \\
\text { Maßnahmen }\end{array}$ & $\begin{array}{l}\text { Welche Ermittlungsmaßnah- } \\
\text { men wenden Sie zur Aufklärung } \\
\text { von Doping-Fällen regelmäßig } \\
\text { an? } \\
\text { (Unterschiede bei } \$ 2 \text { und } \$ 3 \text { ?) }\end{array}$ & \\
\hline TKÜ & 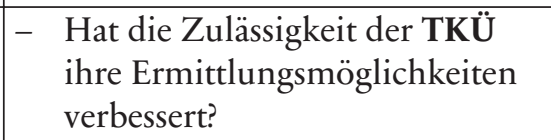 & \\
\hline Kronzeugenregelung & $\begin{array}{ll}- & \text { Wie wichtig ist die bereits exis- } \\
\text { tierende, auf gewerbs- oder ban- } \\
\text { denmäßige Fälle des } ₫ 2 \text { Abs. } 1 \\
\text { und } 2 \text { beschränkte" Kronzeu- } \\
\text { genregelung? } \\
\text { - } \\
\text { Ist die Erweiterung der Kronzeu- } \\
\text { genregelung für Ihre Arbeit not- } \\
\text { wendig? }\end{array}$ & \\
\hline $\begin{array}{l}\text { Bewertung und } \\
\text { Empfehlungen }\end{array}$ & $\begin{array}{l}\text { - Welche neuen Ermittlungs- } \\
\text { möglichkeiten hat Ihnen das } \\
\text { AntiDopG gegeben? Waren sie } \\
\text { sinnvoll und ausreichend? Wel- } \\
\text { che zusätzlichen rechtlichen } \\
\text { Befugnisse wären sinnvoll, um } \\
\text { Doping-Fälle effektiv aufklären } \\
\text { zu können? }\end{array}$ & \\
\hline $\begin{array}{l}\text { Beweisschwierig- } \\
\text { keiten }\end{array}$ & 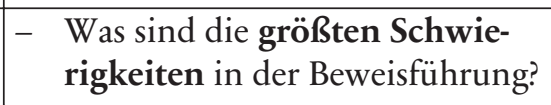 & \\
\hline $\begin{array}{l}\text { SchwerpunktStA/ } \\
\text {-gericht }\end{array}$ & \begin{tabular}{|cc}
- & Was ist der Vorteil einer Schwer- \\
& punktStA? Halten Sie die Schaf-
\end{tabular} & \\
\hline
\end{tabular}

* \46b StGB: Voraussetzung ist eine im Mindestmaß erhöhte Strafe. Das ist nur bei Banden/Gewerbsmäßigkeit der Fall. 
fung von SchwerpunktStA für sinnvoll?

- Halten Sie die Schaffung von Schwerpunktgerichten für sinnvoll?

\section{Teil 3: Verfahrensabschlüsse}

- Wie enden Verfahren nach $\$ 2$ meistens? Gibt es Besonderheiten gegenüber anderen Delikten?

- Wie enden Verfahren nach $\$ 3$ meistens? Besonderheiten gegenüber anderen Delikten?

\begin{tabular}{|c|c|c|}
\hline \multicolumn{3}{|c|}{$\begin{array}{l}\text { Teil 4: Informationsaustausch mit der NADA } \\
\text { Welche Rolle spielt der Informationsaustausch n }\end{array}$} \\
\hline $\begin{array}{l}\text { Was möchten wir } \\
\text { wissen? }\end{array}$ & Weitere Fragen & Notizen \\
\hline Praktische Abläufe & $\begin{array}{l}\text { - Wie läuft die Zusammenarbeit } \\
\text { praktisch ab? } \\
\text { - Wie häufig übermitteln Sie Infor- } \\
\text { mationen und aus welchen Grün- } \\
\text { den entscheiden Sie sich dafür? } \\
\text { (grundsätzlich oder Prüfung im } \\
\text { Einzelfall - nach welchen Kriteri- } \\
\text { en?) }\end{array}$ & \\
\hline $\begin{array}{l}\text { Reaktionen der } \\
\text { NADA }\end{array}$ & $\begin{array}{l}\text { - Gab es in den Fällen, in denen } \\
\text { Sie Informationen geliefert ha- } \\
\text { ben, Konsequenzen von Seiten } \\
\text { der NADA? Ist das der Regelfall? }\end{array}$ & \\
\hline$\frac{\text { Bewertung und }}{\text { Empfehlungen }}$ & $\begin{array}{l}\text { Wie bewerten Sie die einschlägi- } \\
\text { gen Gesetzesänderungen? Haben } \\
\text { Sie Verbesserungsempfehlungen } \\
\text { oder halten Sie die Regelung für } \\
\text { ausreichend? }\end{array}$ & \\
\hline
\end{tabular}




\section{Rechtliche Fragen}

\begin{tabular}{|c|c|c|}
\hline \multicolumn{3}{|c|}{$\begin{array}{l}\text { Teil 1: Rechtliche Fragen zu } \$ 2 \text { AntiDopG } \\
\text { Das AntiDopG hat arzneimittelrechtliche Strafvorschriften in teils erwei- } \\
\text { terter und veränderter Form übernommen. Wir möchten mit Ihnen über } \\
\text { diese, in } \$ 2 \text { zusammengefassten Tatvarianten sprechen, bevor wir auf das } \\
\text { Selbstdoping zu sprechen kommen. } \\
\text { Wie wichtig war die Neukodifzierung für Ihre Arbeit? Können Sie einen } \\
\text { illustrativen Fall nennen, in denen die Neuregelung für Ihre Arbeit prak- } \\
\text { tisch wichtig gewesen ist? }\end{array}$} \\
\hline \multicolumn{3}{|c|}{\begin{tabular}{l|l|l}
$\begin{array}{l}\text { Was möchten wir } \\
\text { wissen? }\end{array}$ & Weitere Fragen & Notizen \\
\end{tabular}} \\
\hline $\begin{array}{l}\text { Überführung der } \\
\text { Tatbestände aus } \\
\text { dem AMG in das } \\
\text { AntiDopG }\end{array}$ & $\begin{array}{l}\text { - Haben die einschlägigen Straftat- } \\
\text { bestände durch die Übernahme } \\
\text { in das AntiDopG eine neue } \\
\text { symbolische und praktische } \\
\text { Bedeutung erhalten? }\end{array}$ & \\
\hline $\begin{array}{l}\text { Erweiterung des } \\
\text { Anwendungsbereichs } \\
\text { um neue Tathand- } \\
\text { lungen }\end{array}$ & $\begin{array}{l}\text { - Das AntiDopG hat die Verbote } \\
\text { um neue Begehungsweisen er- } \\
\text { weitert (herstellen, Handel trei- } \\
\text { ben, veräußern, abgeben, in oder } \\
\text { durch den Geltungsbereich die- } \\
\text { ses Gesetzes zu verbringen): } \\
\text { - Wie beurteilen Sie diese Er- } \\
\text { weiterungen? } \\
\text { - Können Sie einen illustrati- } \\
\text { ven Fall nennen, in dem die } \\
\text { Gesetzesänderung einen } \\
\text { rechtlichen Unterschied ge- } \\
\text { macht hat, zB eine abwei- } \\
\text { chende Verfahrensbeendi- } \\
\text { gung bedingt hat? } \\
\text { Wie wichtig und wie prakti- } \\
\text { kabel sind die Fahrlässig- } \\
\text { keitstatbestände } ₫ 4 \text { Abs. } 6 \text { ? } \\
\text { Ist die Einschränkung der } \\
\text { Besitzstrafbarkeit nach } ₫ 2 \\
\text { Abs. } 3 \text { auf Fälle „nicht ge- }\end{array}$ & \\
\hline
\end{tabular}




\begin{tabular}{|c|c|}
\hline & $\begin{array}{l}\text { ringer Mengen" Ihres Er- } \\
\text { achtens angemessen? }\end{array}$ \\
\hline $\begin{array}{l}\text { Besonderer schwerer } \\
\underline{\text { Fall zu Qualifikationen }}\end{array}$ & 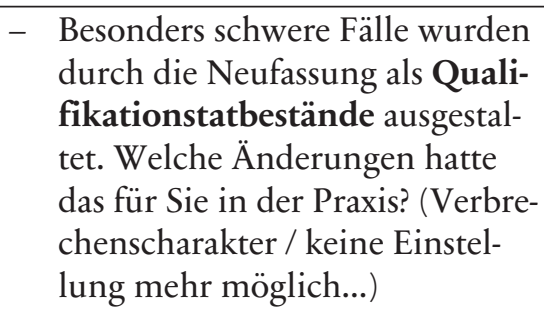 \\
\hline $\begin{array}{l}\text { Mögliche Vortat } \\
\text { für Geldwäsche }\end{array}$ & $\begin{array}{l}\text { - Hat sich in der Praxis etwas da- } \\
\text { durch geändert, dass die Taten } \\
\text { jetzt mögliche Vortaten für die } \\
\text { Geldwäsche sind? }\end{array}$ \\
\hline $\begin{array}{l}\text { Ausdrückliche Erfas- } \\
\text { sung der unerlaubten } \\
\text { Anwendung von } \\
\text { Dopingmethoden }\end{array}$ & 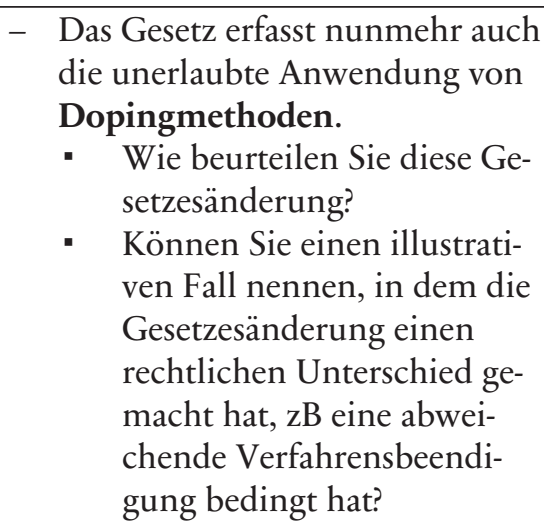 \\
\hline $\begin{array}{l}\text { Bewertung und } \\
\text { Empfehlungen }\end{array}$ & 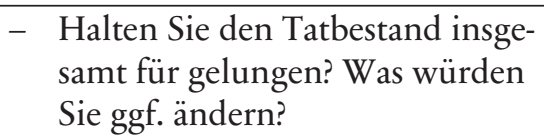 \\
\hline
\end{tabular}




\begin{tabular}{|c|c|c|}
\hline \multicolumn{3}{|c|}{$\begin{array}{l}\text { Teil 2: Rechtliche Fragen zu } ₫ 3 \text { AntiDopG } \\
\text { Haben sich rechtliche Probleme bei der Anwendung des neuen Rechts } \\
\text { zum Selbstdoping gezeigt? Welche? }\end{array}$} \\
\hline $\begin{array}{l}\text { Was möchten wir } \\
\text { wissen? }\end{array}$ & Weitere Fragen & Notizen \\
\hline $\begin{array}{l}\text { Selbstdoping: Allg. } \\
\text { Einschätzung }\end{array}$ & $\begin{array}{l}\text { - Sind die das Selbstdoping betref- } \\
\text { fenden Straftatbestände ausrei- } \\
\text { chend präzise formuliert und } \\
\text { praktisch handhabbar? }\end{array}$ & $\begin{array}{l}\text { Probleme } \\
\text { Spitzen- } \\
\text { sportler? } \\
\text { Einnah- } \\
\text { men? }\end{array}$ \\
\hline Adressatenkreis & $\begin{array}{l}\text { Hat sich der von } \$ 4 \text { Abs. } 7 \text { ge- } \\
\text { wählte Zuschnitt des Adressaten- } \\
\text { kreises praktisch bewährt? } \\
\text { - Wie bestimmen Sie den Be- } \\
\text { griff „Einnahmen erhebli- } \\
\text { chen Umfangs“? }\end{array}$ & \\
\hline Objektiver Tatbestand & $\begin{array}{l}\text { - } \\
\text { An welcher Stelle des objektiven } \\
\text { Tatbestandes stellen sich häufiger } \\
\text { rechtliche Fragen (Normadres- } \\
\text { sateneigenschaft, fehlende medi- } \\
\text { zinische Indikation)? Haben Sie } \\
\text { dafür ein illustratives Bsp.? }\end{array}$ & \\
\hline Subjektiver Tatbestand & 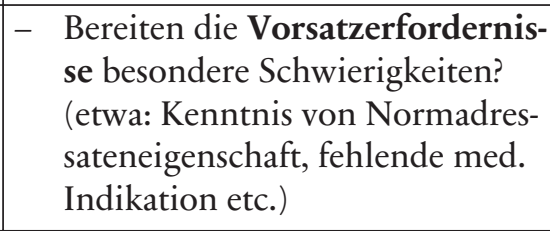 & \\
\hline $\begin{array}{l}\text { Erwerbs- und Besitz- } \\
\text { strafbarkeit ohne } \\
\text { mengenmäßige } \\
\text { Beschränkung }\end{array}$ & $\begin{array}{ll}- & \text { Hat sich die Kriminalisierung des } \\
\text { Erwerbs bzw. Besitzes auch ge- } \\
\text { ringer Mengen zum Zweck des } \\
\text { Selbstdopings praktisch bewährt? }\end{array}$ & \\
\hline $\begin{array}{l}\text { Fehlende Versuchs- } \\
\text { strafbarkeit hinsicht- } \\
\text { lich des Erwerbs bzw. } \\
\text { Besitzes }\end{array}$ & $\begin{array}{l}\text { - } \begin{array}{l}\text { Ist das Fehlen der Versuchs- } \\
\text { strafbarkeit angemessen oder } \\
\text { sehen Sie darin eine Privilegie- } \\
\text { rung bestimmter Sportler? }\end{array} \\
\end{array}$ & \\
\hline $\begin{array}{l}\text { Bewertung und } \\
\text { Empfehlungen }\end{array}$ & $\begin{array}{l}\text { - Halten Sie den Tatbestand insge- } \\
\text { samt für gelungen? Was würden } \\
\text { Sie ggf. ändern? }\end{array}$ & \\
\hline
\end{tabular}




\begin{tabular}{|c|c|c|}
\hline \multicolumn{3}{|c|}{$\begin{array}{l}\text { Teil 3: Strafrahmen und Verjährung } \\
\text { Wie beurteilen Sie die Vorschriften zum Strafrahmen und zur Verjäh- } \\
\text { rung? }\end{array}$} \\
\hline $\begin{array}{l}\text { Was möchten wir } \\
\text { wissen? }\end{array}$ & Weitere Fragen & Notizen \\
\hline Strafrahmen & $\begin{array}{l}\text { - Sind die Strafrahmen Ihrer An- } \\
\text { sicht nach stimmig und ange- } \\
\text { messen? Halten Sie die Privilegie- } \\
\text { rung für Spitzensportler beim Straf- } \\
\text { rabmen der Besitzstrafbarkeit für } \\
\text { angemessen? }\end{array}$ & \\
\hline Verjährung & $\begin{array}{ll}- & \text { Sind die Verjährungsfristen an- } \\
& \text { gemessen lang? }\end{array}$ & \\
\hline $\begin{array}{l}\text { Bewertung und } \\
\text { Empfehlungen }\end{array}$ & $\begin{array}{l}- \text { Halten Sie die Regelungen des } \\
\text { AntiDopG in diesen Punkten für } \\
\text { gelungen? Welche Änderungen } \\
\text { würden Sie ggf. empfehlen? }\end{array}$ & \\
\hline
\end{tabular}

\section{E. Gerichtsverfahren}

\section{Gerichtsverfahren}

Welche Erfahrungen haben Sie in den anschließenden Gerichtsverfahren gemacht?

\begin{tabular}{|c|c|c|}
\hline $\begin{array}{l}\text { Was möchten wir } \\
\text { wissen? }\end{array}$ & Weitere Fragen & Notizen \\
\hline$\underline{\text { Dauer }}$ & $\begin{array}{l}\text { - Wie lange dauern Gerichtsver- } \\
\text { fahren in etwa? Gibt es Punkte, } \\
\text { die zu Verzögerungen führen? }\end{array}$ & \\
\hline Beweisprobleme & $\begin{array}{l}\text { - Welche Herausforderungen zeig- } \\
\text { ten sich bei der Beweiserhebung } \\
\text { in der Hauptverhandlung? }\end{array}$ & \\
\hline$\underline{\text { Deals }}$ & $\begin{array}{ll}- & \text { Gab es Verständigungen? } \\
- & \text { Was waren die Beweggründe der } \\
& \text { StA für die Verständigung? } \\
- & \text { Auf wessen Initiative beruhten } \\
& \text { diese Verständigungsgespräche? }\end{array}$ & \\
\hline
\end{tabular}




\section{F. Allgemeine Einschätzungen}

\begin{tabular}{|l|l|l|}
\hline \multicolumn{3}{|l|}{ Teil 1: Haltung zur Strafbarkeit von Selbstdoping } \\
In der Literatur wird die Strafbarkeit von Selbstdoping teilweise kritisch \\
gesehen. Es wird zB argumentiert, das Gesetz führe zu einer „gewaltigen \\
Überkriminalisierung des Amateursports“ oder verwende zu viele unbe- \\
stimmte Rechtsbegriffe. Können Sie diese Sichtweise kommentieren? \\
\hline $\begin{array}{l}\text { Was möchten wir } \\
\text { wissen? }\end{array}$ & Weitere Fragen & Notizen \\
\hline Innere Haltung & $-\begin{array}{l}\text { Haben Sie den Eindruck, dass die } \\
\text { Strafverfolgung des unerlaubten } \\
\text { Umgangs mit Dopingmitteln } \\
\text { bzw. der unerlaubten Anwen- } \\
\text { dung von Dopingmethoden (1) } \\
\text { generell sinnvoll ist und (2) kön- } \\
\text { nen Sie sich als Strafverfolger mit } \\
\text { der Zielsetzung identifizieren? }\end{array}$ & \\
\hline
\end{tabular}

Teil 2: Allgemeine Bewertung des Gesetzes

Wie bewerten Sie als Praktiker oder Praktikerin das AntiDopG? Ist die Regelung insgesamt "geglückt"?

\begin{tabular}{|l|l|l|}
\hline $\begin{array}{l}\text { Was möchten wir } \\
\text { wissen? }\end{array}$ & Weitere Fragen & Notizen \\
\hline $\mathbb{2} \underline{\underline{3}}$ & $-\ldots$ mit Blick auf $\mathbb{2}$ AntiDopG? & \\
\hline $\mathbb{3}$ & $-\ldots$ mit Blick auf $\mathbb{3}$ AntiDopG? & \\
\hline
\end{tabular}

\section{Teil 3: Konkrete Reformvorschläge}

Was sind aus Ihrer Sicht die dringlichsten Verbesserungsmöglichkeiten auf tatbestandlicher, prozessualer oder praktischer Ebene?

\begin{tabular}{|l|l|l|}
\hline $\begin{array}{l}\text { Was möchten wir } \\
\text { wissen? }\end{array}$ & Weitere Fragen & Notizen \\
\hline Tatbestandlich & & \\
\hline Prozessual & & \\
\hline Praktisch & \\
\hline (institutionell etc.) & & \\
\hline
\end{tabular}


Gibt es noch etwas, das Ihrer Ansicht nach für unser Forschungsprojekt von Bedeutung wäre und das in diesem Gespräch noch nicht thematisiert wurde? 
The act against doping in sport in practice An evaluation 


\section{Table of Content}

A. Fundamentals of the study 175

I. Background and objectives of the evaluation 175

II. Methodological implementation 178

1. Evaluation of case files 179

a) Sample 179

b) Quantitative and qualitative coding 180

c) Evaluation 181

2. Interviews with experts 182

a) Sample $\quad 182$

b) Design of the interview guidelines 183

c) Conducting and evaluating the interviews 184

B. Results of the evaluation 185

I. The prohibition of self-doping (section 3) 185

1. Manifestations of self-doping 185

a) Sports concerned 185

b) Information on the accused 186

c) Doping substances and methods 187

2. Substantive legal issues 188

a) Criminal acts $\quad 188$

aa) Legislative context 188

bb) Quantitative evaluation $\quad 189$

cc) Doping methods 189

dd) The precision and comprehensibility of the formulation of the offence 190

ee) Criminalisation of the purchase and possession of
doping substances, irrespective of quantity

ff) Summary 193

b) Further conditions of criminal liability 194

aa) Medical indication 194

(1) Legislative context 194

(2) Findings of the study 194 
bb) Intention of gaining an advantage in a competition of organised sport

(1) Interpretation of the characteristic "competition of organised sport"

(2) Intention of gaining an advantage

198

(3) Interim result

199

c) Restrictions of criminal liability in section 4 (7) 200

aa) Athletes included in a Registered Testing Pool 200

bb) Significant revenue 201

(1) Teleological appropriateness of the restriction of criminal liability

(2) Difficulties of application

202

(3) Definition and determinability of the formulation 203

(4) Investigations 205

(5) Summary 206

cc) General assessment of section 4 (7) 207

(1) Classification

(2) Reason for low case numbers and rare indictments

(3) Unequal treatment of athletes 208

d) Active repentance 209

aa) Legislative context 209

bb) Results of the study 210

3. Findings on procedural issues 211

a) Knowledge obtainment 211

aa) Ways of obtaining knowledge 211

bb) Leniency 213

b) Investigation measures 213

c) Completion of proceedings 215

aa) No convictions for self-doping 216

bb) Terminations in accordance with section 170 (2) StPO or section 153 StPO 217

cc) Termination because of the absence of proof of intent regarding the use of doping substances 219

dd) Termination without apparent reasons 223

d) Summary penalty orders and sentencing 224

4. Evaluation 225

a) General evaluation of the criminal law regulation of selfdoping

aa) Overview 
bb) Appropriateness of the criminalisation of self-doping $\quad 225$

cc) Fear of deliberate false accusations 228

dd) Concern about the importance of sports jurisdiction $\quad 228$

ee) General evaluation 229

b) Practical proposals for the reform of the criminal law provisions on self-doping 230

aa) Introduction of a specific leniency 230

bb) Improved protection of whistleblowers 232

II. The prohibition norms of section 2

1. Phenomenon 235

a) Findings on perpetrators and criminal acts 235

b) Findings on doping substances 237

c) Summary 238

2. Findings on substantive aspects of section 2

a) The offences and their prerequisites 238

aa) Legislative context 238

bb) Manufacturing, trafficking, placing on the market, prescribing (section 2(1)) 241

cc) Administration or application of doping substances and methods (section 2 (2)) 243

dd) Acquisition, possession and transfer pursuant to section 2 (3)

(1) General meaning 244

(2) Acquisition and possession of significant quantities

(3) Carrying to or through the territory governed by this act

ee) For the purpose of doping human beings in sport

ff) Criminal liability for negligence in accordance with section 4 (6)

b) Penalty enhancing offences 252

c) Summary 254

3. Findings on procedural issues 254

a) Initial suspicion 254

b) Investigation measures $\quad 256$

c) Conclusion of the proceedings 258

d) Characteristics of the court proceeding 260

4. Evaluation 262

a) General assessment 262

b) Legislative implementation 263 
c) Suggestions for improvement 264

aa) Substantive law 264

bb) Procedural law and investigatory powers 266

III. Overarching aspects 267

1. Sentencing range and limitation period 267

a) Sentencing range contained in section $4 \quad 267$

aa) Increasing the maximum sentence contained in section 4 (1) 269

bb) Inconsistency of the applicable sentencing ranges? $\quad 270$

b) Limitation period 271

c) Assessment 272

aa) Maximum sentence 272

bb) Criminal liability for purchase and possession 273

cc) Limitation period 273

2. Establishment of specialised public prosecutor's offices and courts 273

a) The demand of the NADA and the athlete's associations 274

b) On the establishment of specialised public prosecutor's offices 275

aa) The perspective of the judiciary 275

bb) Findings from the evaluation of files 277

c) On the establishment of specialised courts 278

d) Assessment 280

3. Cooperation with the NADA and section $8 \quad 282$

4. Training 284

C. Final opinion of the experts 287

I. General assessment 287

1. Self-doping (section 3) 287

2. Unlawful handling of doping substances and unlawful application of doping methods (section 2) 288

II. Specific recommendations of the experts 289

1. Substantive amendments 289

a) Self-doping 289

b) Unlawful handling of doping substances (section 2) 292

2. General procedural and institutional changes 295

a) Leniency and whistleblower systems 295

b) Specialised public prosecutor's offices and specialised
courts 
Annex: Guideline for public prosecutors

\section{References}

1. All paragraphs without further specification refer to the act against doping in sport (AntiDopG).

2. The occasional use of the generic masculine is due to the readability of the study and includes persons of all genders. 


\section{A. Fundamentals of the study"}

\section{Background and objectives of the evaluation}

The "Act to Combat Doping in Sport" (AntiDopG) of 10 December 2015 is intended to protect the integrity of sport, the fairness and equal opportunities in competition and the health of athletes by making users of doping substances and doping methods as well as perpetrators of various forms of preparation liable to prosecution (cf. section 1 AntiDopG ${ }^{1}$ ). The AntiDopG is expected to provide a "negative counter-incentive" to compensate for the "doping affinity" that goes hand in hand with the growing commercialisation of sport. ${ }^{2}$ In addition to this deterrent effect, the punitive prohibitions of the AntiDopG are also said to have a general preventive effect: They are supposed to contribute to anchoring the prohibition of doping, especially self-doping, in social ethics more strongly and more deeply than before. This is intended to support the efforts of sports associations which - unlike the State - lack important means to enforce the doping ban. ${ }^{3}$

In the opinion of the legislature, the rules that were in force until December 2015 had proved to be inadequate, as the doping-specific criminal regulations that existed at that time had gaps, particularly with regard to preparatory acts, the possession of small quantities of doping substances for self-administration and - especially - with regard to the so-called selfdoping ${ }^{4}$. The AntiDopG therefore not only adopted the existing regulations of the Medicinal Products Act (AMG) and thus made them more visible - which could be useful for general prevention. The law has also been supplemented by further prohibitions or certain modalities of existing offences, in particular by the prohibition of self-doping in competitions of organised sport, by new offences including preparatory acts and the extension of aggravated offences, which are linked to more extensive powers of

* An English version of the Anti-Doping Act can be found on the website of the Federal Ministry of Justice: http://www.gesetze-im-internet.de/englisch_antidopg/inde x.html.

1 In the following, all paragraphs not specified in detail are those of the AntiDopG.

2 See also Rössner, in: Lehner/Nolte/Putzke, AntiDopG, 2017, Vor $\$ 1$ Rn. 30.

3 Already Steiner, NJW 1991, 2729, 2733.

4 BT-Drs.18/4898, p. 2. 


\section{A. Fundamentals of the study}

investigation. The AntiDopG has thus expanded the scope of prohibited conduct and the possibilities for enforcing the law through criminal proceedings. Furthermore, it has also changed the existing functional-institutional arrangement: This applies in particular to the exchange of information between the National Anti-Doping Agency (NADA) and the public prosecutor's offices. In addition, informal effects may occur: It is likely that associations (and clubs) have adapted their preventive measures, since athletes, trainers and other persons active in organised sport are faced with new risks related to criminal law. As a result, the law should have gained an additional preventive effect.

Almost five years after the AntiDopG came into force, the question arises as to how the new options are used in practice and whether the AntiDopG has come closer to achieving its stated goals.

In the legal literature this question is not appraised in a consistent manner. While some are decidedly "pessimistic" in view of the comparatively small number of completed criminal proceedings, ${ }^{5}$ others refer to a positive preventive effect, which cannot be measured by the number of proceedings. ${ }^{6}$ Some athletes are concerned that the breadth of the criminal offences could lead to invasive investigations that eventually lead nowhere. ${ }^{7}$ Investigators respond that the AntiDopG makes it possible to conduct targeted investigations; the initially small number of criminal proceedings, they argue, is not unusual but is due to the novelty of the law and the nature of the proceedings. ${ }^{8}$

Our study serves as a basis for the evaluation of the effectiveness of the new AntiDopG, as required by its Article 8. It is intended to provide scientific support for the determination and evaluation of the effects of the new law on the criminal prosecution of doping violations and to identify possible weaknesses that become apparent in the application of the law in order to enable improvement measures. The study is therefore focused on the question of whether and how the legal changes brought about by the AntiDopG have affected legal practice. In addition to an investigation of criminal prosecution practice, the study will cover the perception of the legal

5 So Jahn, in: Hoven/Kubiciel, Korruption im Sport, 2018, pp. 117, 123 et. seq.; sceptically already Kreuzer, ZRP 2013, 181, 182 et seq.

6 So Rössner, quoted from Armenat, in: Hoven/Kubiciel (footnote 5) pp. 135 et seq., 139 et seq.

7 Kassner, in: Hoven/Kubiciel (footnote 5), pp. 135 et seq.

8 Supporting documents in Armenat's discussion report, in: Hoven/Kubiciel (footnote 5), pp. 139, 144. 
changes by representatives of external organisations such as the NADA or the athletes associations.

The central guiding questions of the evaluation were:

I. Phenomenon

- Which forms of doping are relevant in the practice of prosecution (type of sport, perpetrator, type of doping, etc.)?

II. Prosecution of self-doping

- How is self-doping investigated and prosecuted in practice?

o How is an initial suspicion generated?

o What investigation measures are conducted?

o What criminal acts are investigated and lead to prosecution and conviction?

o How long do the proceedings take?

o How do suspects react when interrogated?

o How are preliminary investigations concluded - and on what grounds?

o What evidentiary problems arise in preliminary investigations and in court?

- How are the subjective prerequisites for the offence proven, such as the intention to procure an advantage in section 3 (1), (2) or the knowledge that there is no indication in section 3 (2), (4)?

- How can be proven that a doping substance or a doping method was used "without medical indication"?

- Which additional procedural options are considered useful? (e.g.: leniency for providing information on other offenders)

o What legal questions arise in the application of the AntiDopG?

- How is it determined whether the competition is a competition of organised sport?

- How is the circle of addressees described in section 4 (7) understood?

- How is "significant revenue" in section 4 (7) no. 2 determined?

o Are there cases of active remorse in accordance with section 4 (8)?

o What sanctions were imposed?

III. Criminal prosecution of section 2 in conjunction with section 4

- How does the prosecution of section 2 work in practice?

o How is an initial suspicion generated?

o What investigation measures are conducted? 
o What changes occurred in practice due to the introduction of section 2?

- Does the extension of the offence by additional criminal acts play a role?

- Does it matter that the act now also covers the illicit use of doping methods?

- How important and how practicable are the offences by negligence in section $4(6)$ ?

- What impact has the restriction of criminal liability for possession in accordance with section 2 (3) on cases of "significant quantities"?

- Has the introduction of penalty enhancing offences (instead of particularly serious cases) brought about any change in practice?

- Has anything changed in practice thus the acts are now possible predicate offences for money laundering?

o How are the proceedings concluded?

IV. General questions and assessments

- How are the range of sentences and limitation periods assessed?

- How is the establishment of specialised public prosecutor's offices and specialised courts evaluated?

- What significance has the cooperation with the NADA in accordance with section 8 ?

- How do practitioners assess the changes in criminal law and criminal proceedings in the AntiDopG as a whole?

\section{Methodological implementation}

The study exclusively examines detected cases. Since the aim of the study is not to investigate the actual spread of doping, but solely to record the effects of the AntiDopG on the criminal prosecution of doping violations, unreported cases do not play a decisive role for the present investigation.

The following methods were used:

- Qualitative and quantitative evaluation of all case files on self-doping.

- Expert interviews on practical experience with the amended AntiDopG. 
1. Evaluation of case files

a) Sample

In accordance with the guidelines given by the Federal Ministry of Justice and Consumer Protection (BMJV), the evaluation of files is limited to proceedings on self-doping in accordance with section 3. The aim was to collect all relevant files of preliminary investigations since the introduction of the criminal liability of self-doping. The files of ongoing proceedings were excluded, as a first step the BMJV asked the States' Ministries of Justice for information on relevant proceedings in the public prosecutor's offices in their State. Afterwards the Ministries of Justice as well as the reported public prosecutor's offices were contacted again. An application for inspection of the files in accordance with section 476 of the German Code of Criminal Procedure (StPO) was made. All public prosecutors' offices made the files held by them available. Not all files made available to the experts concerned the object of investigation; instead, case files that were conducted exclusively for violations of section 2 were transferred as well.

In total 526 files were made available. Of these, 103 proceedings actually concerned self-doping. Two different types of proceedings were covered: proceedings conducted expressly in accordance with sections 3, 4 and proceedings in which the facts of those sections were not explicitly mentioned, but recognisably examined. Only these proceedings were included in the evaluation. The files are distributed among the various public prosecutors' offices as follows.

\begin{tabular}{|l|l|l|}
\hline Federal state & Made available & Relevant \\
\hline Baden-Württemberg & 35 & 35 \\
\hline Bavaria & 27 & 7 \\
\hline Berlin & 9 & 0 \\
\hline Brandenburg & 19 & 3 \\
\hline Bremen & 5 & 1 \\
\hline Hamburg & 1 & 1 \\
\hline Hesse & 12 & 8 \\
\hline Mecklenburg-Western Pomerania & 21 & 4 \\
\hline Lower Saxony & 39 & 7 \\
\hline North Rhine-Westphalia & 248 & 29 \\
\hline
\end{tabular}




\begin{tabular}{|l|l|l|}
\hline Federal state & Made available & Relevant \\
\hline Rhineland-Palatinate & 7 & 1 \\
\hline Saarland & 1 & 1 \\
\hline Saxony & 71 & 0 \\
\hline Saxony-Anhalt & 5 & 2 \\
\hline Schleswig-Holstein & 12 & 1 \\
\hline Thuringia & 14 & 3 \\
\hline Total & $\mathbf{5 2 6}$ & $\mathbf{1 0 3}$ \\
\hline
\end{tabular}

The low number of cases also corresponds to the assessment of the public prosecutor's offices:

"The application of section 3 has almost not been relevant for us, at least in my field of activity. As far as I know I never had to deal with an accused person in accordance with section 3." (public prosecutor's office (StA) 5)

b) Quantitative and qualitative coding

Some of the files were inspected at the premises of the public prosecutor's offices, while others were sent to the universities of Augsburg or Leipzig. For the coding of the files a qualitative coding sheet was developed in Excel. This was done in coordination with the ministries involved. The coding sheet contained 32 categories on phenomena, investigation and court proceedings as well as the legal and evidence questions raised. The categories were determined by the previously developed research questions, for which detailed sub-questions were formulated. To ensure aspects relevant to the evaluation would not be excluded due to presumptions an open category named "other particularities" was included.

For the quantitative evaluation a codebook was created. The codebook contains numerical recorded data - such as the frequency of the sports concerned or the form of the conclusion of the proceedings.

To ensure the greatest possible conformity, the experts coded the first case files jointly. In the following comparison of the results of the analysis deviations should be detected. In this way, differences in the coding could be disclosed at an early stage and divergences could be eliminated. Only af- 
ter the codings had conformity of more than 90 percent, the files were transferred independently into the analysis sheet. ${ }^{9}$

\section{c) Evaluation}

The findings of the qualitative file analysis were transferred to an overall Excel spreadsheet. Afterwards the table could be evaluated vertically and horizontally. Whereas the vertical evaluation enabled a cross-procedural analysis of certain features and categories, the horizontal evaluation showed concrete proceedings in completeness.

The quantitative data was analyzed using the statistical program IBM SPSS Statistics 26. In accordance with the provisions of the codebook the results of the qualitative data collection were coded in a separate Excel spreadsheet as a first step. The dataset was then imported into SPSS and verified for its completeness. The coding of the information was mostly done in a nominal scaling. Multiple answers were possible in individual cases (e.g. in case of questions about the coercive measures applied). For the determination of time intervals (e.g. the duration of the investigation proceeding) a metric scaling was chosen.

After data preparation, the complete data set $(\mathrm{n}=103)$ was condensed by means of descriptive statistics. It was aimed to obtain measuring values as well as tables and graphs for the research questions mentioned above. ${ }^{10}$ The present results are based on a complete survey of all preliminary investigations conducted in Germany due to initial suspicion of self-doping. Therefore - provided that all relevant case files have been sent - a prognosis using inductive statistics is not necessary.

The data which were scaled nominally were examined for their absolute and relative frequency distribution. To determine the relative frequency, the measured numbers were divided by the entire data set $(n=103)$ as well as by the number of all relevant proceedings ( $\mathrm{n}=$ variable). The measurement numbers were also visualised by pie charts or bar charts, to ensure better illustration. For the data which were scaled metrically the mean as

9 According to Rössler/Geise, the minimum standard for content categories of complex constructs is 80 percent agreement; see Rössler/Geise, in: Möhring/Schlütz (ed.), Handbuch standardisierte Erhebungsverfahren in der Kommunikationswissenschaft, 2013, p. 269.

10 Cf. on the methodological approach Kosfeld/Eckey/Türck (ed.), Deskriptive Statistik, 6th ed., 2016, pp. 37 et seq. 


\section{A. Fundamentals of the study}

well as minimum and maximum values were calculated. Due to the small fluctuations, it was not necessary to determine the median.

\section{Interviews with experts}

On the one hand, the expert interviews were conducted for the purpose of evaluating section 2 , which was not subject of the file investigation. On the other hand, the interviews complemented the file evaluation on self-doping with important aspects: Experience has shown that criminal files are incomplete in their information content and do not include decisive strategies and backgrounds, such as agreements, factual obstacles and defence strategies sufficiently.

\section{a) Sample}

Interviews were conducted with the following experts ${ }^{11}$ :

- 1 representative of the NADA

- 1 representative of the Customs Investigation Office in Frankfurt am Main

- 1 representative of the Main Customs Office in Frankfurt am Main

- 1 representative of the Customs Criminological Office

- 1 representative of the Federal Criminal Police Office (BKA)

- 1 representative of the New Representation of Athletes (Neue Athletenvertretung)

- 1 representative of the representation of Athletes of the German Olympic Sports Federation (DOSB)

- 1 representative of the DOSB

- 2 public prosecutors at Frankfurt am Main

- 2 public prosecutors at specialised offices in Zweibrücken

- 2 public prosecutors at specialised offices in Freiburg

- 1 public prosecutor at a specialised office in Munich ${ }^{12}$

11 In order to prevent conclusions being drawn about the person of the respondent the generic masculine is used for the experts; persons of each sex are covered.

12 A second interview was planned, but did not take place due to scheduling difficulties, partly caused by the Corona pandemic. 
- 1 judge at the Local Court Zweibrücken ${ }^{13}$

- 1 judge at the District Court Munich

The concrete selection of individual interviewees was for the most part done by the authorities or associations. The risk of a strategic selection by the selecting intermediary ${ }^{14}$ appeared to be low. The experts were mainly appointed based on the areas of responsibility within the respective institution. Indeed, the suspicion of a strategic selection occurred in no case. According to the experts' impression, the intermediaries named those experts who had the most experience in the fight against doping in their area of responsibility.

b) Design of the interview guidelines

To obtain answers to all relevant research questions, the interviews were structured by interview guidelines that the experts developed in consultation with the ministries involved. For each group to be interviewed, separate guidelines were developed. However, to ensure comparability of the results, these guidelines essentially contained the same questions, but were tailored to the concrete field of experience of the interviewee.

In the expert interviews controlling and structuring in a greater extent was harmless. It was less focused on the subjective opinion of the respondents about the importance of aspects and more on subject-specific questions. ${ }^{15}$ The use of a guideline ensured the comparability of the interviews in the later analysis. ${ }^{16}$ To do justice to the qualitative principle of openness despite the comparatively high level of structuring, ${ }^{17}$ the guideline contained demands to narrate, which were thematically focused initially but

13 According to the District Court Zweibrücken and the Local Court Zweibrücken only one judge at the Local Court Zweibrücken had significant experiences with the AntiDopG so far. That is why no further interviews were conducted at these courts.

14 Kruse, Einführung in die Qualitative Interviewforschung, 2011, p. 93.

15 Kruse, Einführung in die Qualitative Interviewforschung, 2011, p. 70. On the development of guidelines for expert interviews: Bogner/Littig/Menz, Interviews mit Experten, 2014, p. 27 et seq.

16 Helfferich, in: Baur/Blasius (ed.), Handbuch Methoden der empirischen Sozialforschung, 4th ed. 2014, pp. 559, 566.

17 Hoffmann-Riem, Kölner Zeitschrift für Soziologie und Sozialpsychologie 1980, 325; Kruse, in: Gredig/Schnurr (ed.), Forschen in der Sozialen Arbeit, 2011, p. 158. 


\section{A. Fundamentals of the study}

narratively open. These narratives could possibly be supplemented by more detailed questions. ${ }^{18}$

Example of an open question:

"What investigative and coercive measures do you use to solve doping cases and with what success?"

Example of a possible demand:

"Has the admissibility of the telecommunications surveillance (TKÜ) improved your investigative possibilities?"

The transformation of the general research questions into concrete and comprehensible interview questions ${ }^{19}$ was carried out using the "SPSS" method developed by Helfferich. ${ }^{20}$ The guideline for the interviews with the experts contained various modular content areas on the phenomenon and prosecution of doping. ${ }^{21}$

\section{c) Conducting and evaluating the interviews}

The interviews, which were recorded with the consent of the interviewees, were conducted mostly by telephone and scarcely in personal presence. The recordings of the interviews, which lasted on an average about 30-40 minutes, were then transcribed for evaluation. The evaluation of the interviews was carried out solely qualitatively. The same principles that were applied in the analysis of the files were used. While the guidelines were transmitted into categories the answers were transferred to a common Excel spreadsheet.

18 In this way the principle "as open as possible, as structuring as necessary" formulated by Helfferich was implemented in the design of the guidelines; Helfferich, in: Baur/Blasius, Handbuch Methoden der empirischen Sozialforschung, 4th ed. 2014, pp. 559, 560.

19 Kaiser, Qualitative Experteninterviews, 2014, p. 52.

20 Helfferich, Qualität qualitativer Daten, 2005.

21 As an example, the guidelines for the interviews with the public prosecutor's offices are attached as an annex. 


\section{B. Results of the evaluation}

I. The prohibition of self-doping (section 3)

1. Manifestations of self-doping

The relevant proceedings have the phenomenological characteristics outlined below.

\section{a) Sports concerned}

The investigated proceedings were related in particular to the area of weight training $(20.4 \% 22)$ and bodybuilding $(18.4 \%) .{ }^{23}$ The remaining cases concerned sports such as martial arts (13.6\%), cycling (4.9\%) and athletics $(5.8 \%)$.

22 Percentages are rounded to one decimal place.

23 Weight training includes powerlifting, bench press and weightlifting, whereas bodybuilding focuses on the presentation of the body and not on the performance of strength. 
Fig. 1: Distribution of the sports in the sample investigated

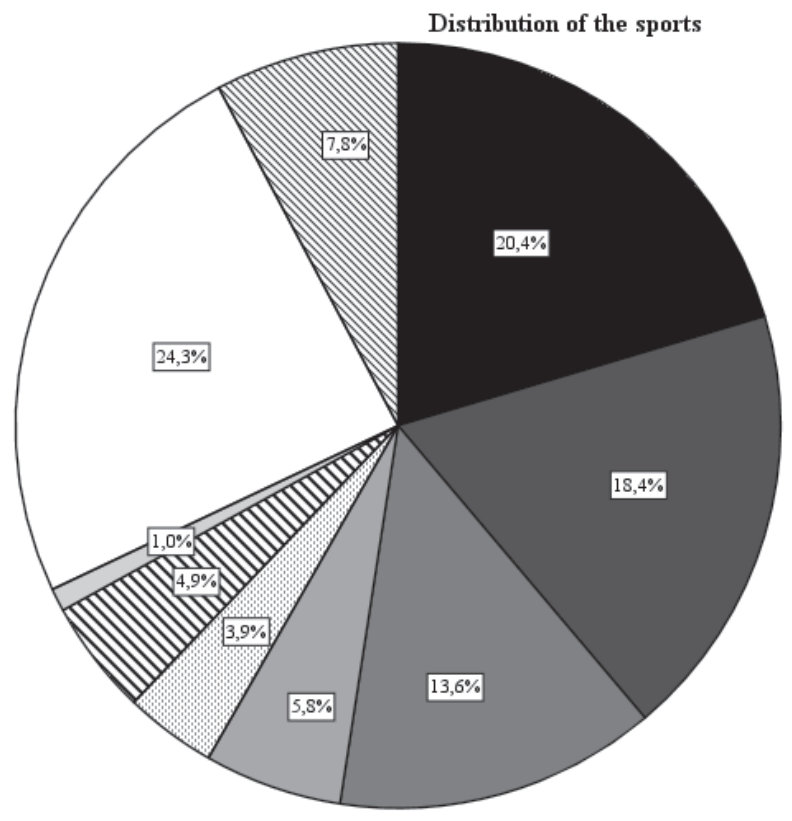

Weight training

Bodybuilding

Martial arts

Athletics

Marathon

Scycling

$\square$ speed skating

$\square$ Other

$\mathbb{N}$ Not specified

Other sports covered by the NADA under risk group $\mathrm{A}^{24}$, such as speed skating, canoeing, swimming, triathlon or rowing, are not included in the sample or are only included in one individual proceeding.

b) Information on the accused

The majority of the accused were male (86.1\%). Most of them were born in the nineties (37.9\%) and eighties (28.2\%). Among the accused were two teenagers and 14 adolescents. Only one proceeding was conducted for participation in self-doping; in the remaining 102 proceedings the accusation was committing the crime as a perpetrator.

24 https://www.nada.de/fileadmin/user_upload/nada/DKS/160729_UEbersicht_Risi kogruppen.pdf (accessed on: 26 May 2020). 
c) Doping substances and methods

The subject of the investigations was in $96.1 \%$ of the proceedings exclusively prohibited doping substances, in $2.9 \%$ of the proceedings doping methods and in $1 \%$ both. The comparatively low significance of doping methods was also confirmed in the interviews.

Questioner: "The law also covers doping methods, not just doping substances. Has this ever been relevant for your work?"

Public prosecutor's office 5: "No. In fact, not at all. Although I currently have two proceedings where doctors might be involved, these doctors possibly administered doping substances without a real medical indication rather than actually using doping methods."

The doping substances most used were anabolic steroids $\left(45.6 \%{ }^{25}\right)$. Diuretics became relevant in only $2.9 \%$ of the cases. Stimulants, including DMAA, which is known as a sports drug, as well as THC, cocaine, ritalin or amphetamine, had significance in $25.2 \%$ of the cases. In $14.6 \%$ of the proceedings, substances were used, which were not intended to have an immediate performance-enhancing effect, but to "mask" the illicit use of e.g. anabolic steroids.

Doping substances and methods in absolute terms:

\begin{tabular}{|l|l|}
\hline Anabolic steroids & 47 \\
\hline - thereof testosterone/ testosterone derivatives & 23 \\
\hline Stimulants & 26 \\
\hline - thereof DMAA & 8 \\
\hline Masking & 15 \\
\hline Diuretics & 3 \\
\hline Method & 4 \\
\hline
\end{tabular}

25 In some cases, different agents were combined (e.g. anabolic steroids and stimulants); therefore, multiple answers are possible. 
2. Substantive legal issues

a) Criminal acts

aa) Legislative context

Section 4 contains a differentiated system of criminal acts. These acts differ in regard to the injustices committed. Section 4 (1) nos. 1 to 3 sanctions violations of the prohibitions contained in section 2 . The criminal acts covered by section 4 (1) nos. 4, 5 and section 4 (2) refer to the self-doping prohibited by section 3. Violations of section 3 constitute different criminal offences. The administration or application of doping substances or doping methods to yourself or the consent to this administration or application in violation with section 3 (1) sentence 1 causes criminal liability in accordance with section 4 (1) no. 4. The participation in a competition of organised sport in violation with section 3 (2) causes criminal liability in accordance with section 4 (1) no. 5. The purchase or possession of a doping substance in violation with section 3 (4) causes criminal liability in accordance with section 4 (2).

Particularly controversial was the criminal liability of self-doping introduced by section 4 (1) no. 4. According to the legislator section 4 (1) no. 4 is "one of the primary cornerstones of the conception of the fight against doping aimed by the Act against doping". ${ }^{26}$ The prohibition of participation in a sporting competition under the influence of doping was introduced in the legislative procedure. Closing gaps in criminal liability, for example in cases in which the perpetrator dopes himself abroad in order to participate in a competition at home, was the intention. ${ }^{27}$ If the doping substances are purchased or possessed for the purpose of self-doping, the possession of small quantities is also criminally liable. According to the explanatory memorandum to the Act, this would be justified, also taking into account the last resort function of criminal law, because already the purchase or possession of doping substances causes a considerable threat to the legally protected goods. This would be the only way to "effectively take action against doping and thus protect the integrity of organised sport." 28

26 BT-Drs. 18/4898, p. 29.

27 Approving MüKo-StGB/Freund, 3rd ed. 2018, AntiDopG $\$ \$ 1$ 1-4 Rn. 36.

28 BT-Drs. 18/4898, p. 29. 
bb) Quantitative evaluation

The evaluation of the files showed the following distribution of the preliminary investigations among the criminal acts related to self-doping.

Fig. 2: Distribution of the proceedings by criminal acts in accordance with section 4

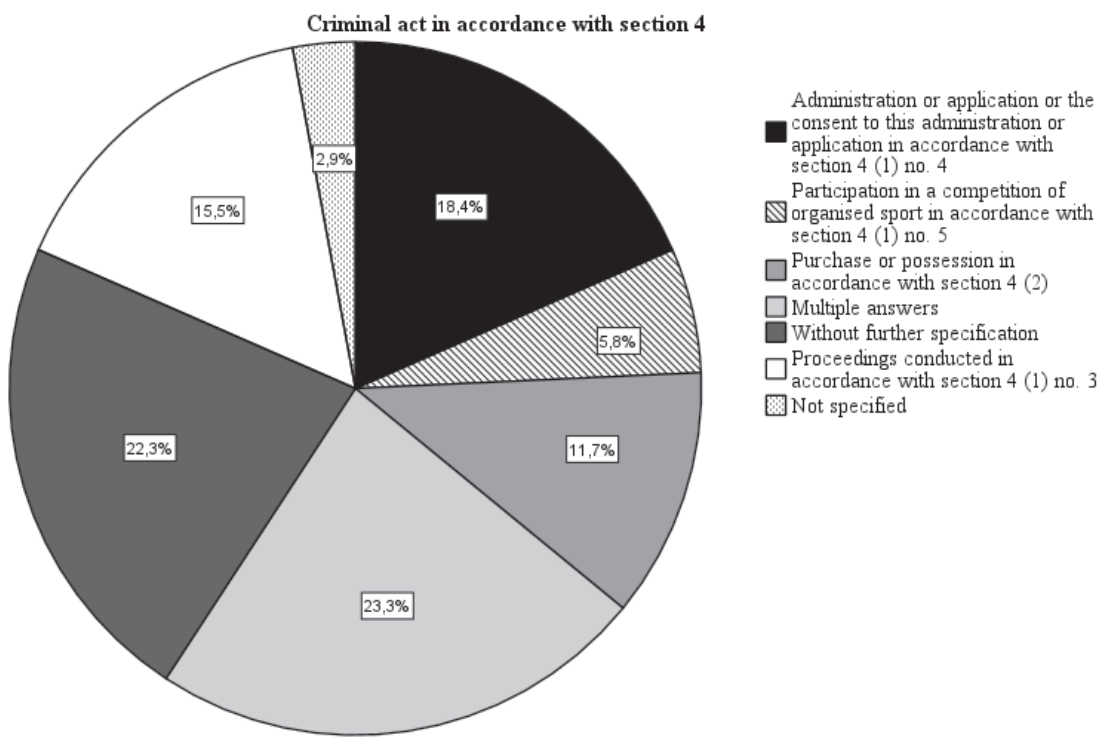

The criminal offence "participation in a competition of organised sport" (section 4 (1) no. 5) is clearly underrepresented. In a significant proportion of proceedings, information on the criminal act was missing or not specified.

cc) Doping methods

It was striking in the evaluation that the doping methods covered by the act have not played a role in the investigations yet (see 1. c) above): The application of doping methods was the exclusive subject matter in only three proceedings $(2.9 \%)$; in one proceeding the subject matter was both 
doping substances and doping methods (1.0\%). Nevertheless, it is generally considered useful to cover doping methods by the act.

"I think it is generally useful." (Athletes association (A) 1)

dd) The precision and comprehensibility of the formulation of the offence

Striking differences also occurred in the answers to the question of whether the offences of self-doping were sufficiently precise and comprehensibly formulated. While the public prosecutor 4, for example, answered the question with "yes" without further ado, a representative of one association implied difficulties in understanding of the athletes or communication with the athletes:

"Of course, this requires supreme legal expertise. (...) I think all these different offences are relatively complicated. However, I think what is allowed and what is not allowed can be explained to athletes very well." (DOSB (D))

In another interview the concerns emerged even stronger:

"In principle, it can be worked with, although the references within the legal norm are sometimes quite difficult as well. We also notice this when we write to public prosecutor's offices that are not specialised. In these cases, it is sometimes-even for an experienced public prosecutor - difficult to read the legal norm and the corresponding references correctly. Therefore, some clarity could surely contribute to legal certainty." (NADA $(N))$

One of the judges interviewed expressed the criticism particularly clear:

"It may be that they are sufficiently precisely formulated. For the application in practice they are very bad. I believe that criminal offences have to be immediately understandable when they are perused for the first time - at least for someone who has been doing criminal law for many years. After all, they have to be understandable to the addressee as well." (Judge (R) 1)

The analysis of the files confirms the concerns partially. As the statistics (above a) show, the public prosecutor's offices did not provide any or no specific information on the criminal act in a conspicuously high number of cases. These proceedings were initiated, and in some cases continued, with a simple reference to "section 4 AntiDopG", "section 4 (1) AntiDopG” or even "violation of AntiDopG”. It was not recognizably referred 
to a specific criminal offence contained in section 4 or at least distinguished between a violation of the prohibitions of section 2 and a case of self-doping in accordance with section 3 . In view of the fact that the different criminal offences of section 4 differ regarding the injustice committed and that different legal prerequisites and a different range of sentences apply, such a procedure is problematic. Accordingly, the investigations often did not proceed accurately and were quickly stopped. Multiple answers regarding the criminal acts are not unproblematic as well: In a not insignificant number of files formulations reminding of text modules such as "selfdoping; application or the consent to the application of doping substances; participation in competition", sometimes combined with "purchase or possession in violation of section 3 (4)," were used. Such formulations only conceal the fact that the concrete reference point of the investigations is actually unclear.

That the system of the criminal facts with its diverse protective purposes and conditions of application is not always understood to the necessary extent is also suggested by another finding. In some cases, public prosecutor's offices justified the initial suspicion of a possession offence in accordance with section 4 (2) simply by the fact that a doping substance was ordered. Neither was the sport practised identifiable nor findings of participation in competitions or income available. On the ground that the offence was committed only once and that the guilt was minor the proceedings were then stopped - usually without further legal explanations. In one proceeding the accused was informed:

"Since the possible fault seems to me relatively small and I assume that this is solely a one-time violation of a criminal law, I refrain from further prosecution for once. However, I strongly reprimand the behaviour shown. In case of recurrence, you cannot expect the termination of the proceedings again." (Excerpt from file)

Such a justification for the termination of the proceedings enables the accused neither to comprehend the reason for the criminal proceedings nor the contours of the criminal conduct, so that goals pursued by special prevention are missed. Above all, the justification for the termination of the proceedings provides grounds for doubting whether the public prosecutor has dealt with the legal requirements in the necessary differentiation.

Finally, the public prosecutor's offices applied section 4 (1) no. 3 in 17 cases, even though the content of the investigations was self-doping. 
ee) Criminalisation of the purchase and possession of doping substances, irrespective of quantity

If it serves the purpose of self-doping (section $3(4))$ the purchase or possession of even a small quantity of doping substances is liable to prosecution in accordance with section 4 (2). During the legislative process, the concern that a quantity-independent criminalisation of the purchase and possession of doping substances could lead to preliminary investigations caused by mistakes of the athlete or his medical or sporting environment in the procurement of medicines or to preliminary investigations caused by the deliberate placing of doping substances near the athlete by a competitor in order to harm him has occasionally been expressed. ${ }^{29}$ These concerns were not confirmed during the evaluation. An athlete's representative said:

"So far I am not aware of any of the cases described and therefore I want to say at this point that the concerns have not been confirmed." (A 2)

However, it is unclear whether the criminalisation of small quantities is also necessary in practice. A public prosecutor of a specialised public prosecutor's office said:

"In my practice I am not aware of any case where that was relevant." (StA 4)

Another investigator considers that the criminalisation of the purchase and possession of even small quantities of doping substances is at least in the case of top athletes not relevant, as these doping substances are typically not procured by the athletes themselves, but are administered by others (e.g. sports doctors) in their practice or at another location:

"Usually they do not own it themselves, but are actually given it for consumption or are actually provided with it under medical supervision." (StA 3)

Nevertheless, public prosecutors were mostly in favour with the modification of the law:

"I think that the criminalisation of small quantities in case of a competitive athlete has proven itself in practice. On the one hand, it was aimed by the

29 See, for example, Opinion on the Act against doping in sport by the Athletes' Commission of the DOSB, 2015, p. 2. See also Peukert, npoR 2015, 95, 99. 
legislator as well as by the purpose of the AntiDopG and has also proven itself regarding my feelings and perceptions. It simplifies the criminal prosecution of the competitive athlete, because the initial suspicion can be affirmed faster, in order to take all measures in the criminal proceedings. If one has cases in this area, this formulation is sufficient to justify an initial suspicion. On the other hand, in the field of weight training, there is no initial suspicion of a competitive athlete, since the possession of doping substances exceeds a small quantity." (StA 3)

"I consider it to be useful that the athlete must not possess any doping substances at all." (StA 1)

On the contrary, one public prosecutor raised concerns:

"We apply section 153 or section 153a StPO, which provide the possibility to refrain from imposing criminal penalties in case of insignificant guilt. I think there's too much criminalisation, which shouldn't necessarily be the case. Maybe the not small quantities should be adjusted again." (StA 2)

\section{ff) Summary}

To sum up, doubts concerning the way the legislative formed the Act are noticeable. They are confirmed quantitatively as well as by two problematic patterns that arose in the conduct of the investigations. Therefore, consideration should be given to simplifying the structure of the offence, in particular by a clearer distinction between those alternatives of the offence that refer to violations of section 2 and those that refer to self-doping. The application of the restriction related to the offender by subsection 7 for those alternatives of the offence that refer to self-doping makes a clear distinction even more necessary. Adding the difficulties caused by the restriction of the group of perpetrators under section 4 (7) (see 2., b., bb below), a more precise definition of the criminal offences should be considered (see C II 1 a below). 
b) Further conditions of criminal liability

aa) Medical indication

(1) Legislative context

Doping means the use of doping substances and methods for an intervention in the body that is not medically indicated. ${ }^{30}$ Since many doping substances are (authorized) drugs at the same time, the AntiDopG must distinguish between inadmissible and prohibited applications of such drugs. In accordance with section 3 (1), self-doping is therefore only prohibited and in accordance with section 4 (1) no. 4 liable to prosecution if an athlete uses or allows the use of doping substances or methods "without medical indication". If a doping substance or method is used for therapeutic purposes, the behaviour is socially acceptable, even if the athletic performance increases as a side effect. ${ }^{31}$ This shows the duality of the protective directions of the AntiDopG, which not only protects the integrity of sport but also the health of the athletes (cf. section 1). However, where the use of drugs is medically indicated, there is no room for prohibition or even punishment. In the legal literature, the distinction between medically indicated and non-indicated use is considered "difficult". ${ }^{32}$

(2) Findings of the study

For such difficulties the evaluation has revealed isolated indications. However, the investigations focused more often on other elements and the pro-

30 See BT-Drs. 18/4898, p. 22; Weber BtMG/ ibid. 5th ed. 2017, AntiDopG $\mathbb{3}$ Rn. 18.

31 Cf. BT-Drs. 18/4898, p. 27 - Under sports law the granting of a Therapeutic Use Exemption (TEU) for the use of prohibited substances or methods in accordance with the World Anti Doping Code (WADC) is required for such cases; for details see the International Standard for Therapeutic Use Exemptions. The taking of non-prohibited drugs or substances has to be reported on the doping control form during doping control.

32 Lehner/Nolte/Putzke/Putzke, Anti-Doping-Gesetz, $\mathbb{3}$ Rn. 10, on the grounds that after illnesses or accidents prescribed medications always (and possibly up until competitions) had a performance-enhancing effect; this fails to recognise that the prohibition is not based on performance enhancement but on the medical indication. What is medically indicated is decided by the state of medical science; in addition, special permits (TEU) can be obtained (see footnote above). 
ceedings were closed for other reasons (minor guilt, foreign facts). Only in one interview the medical indication was mentioned as a problematic point in the investigations:

"Most of the time it is certainly a lack of medical indication, because in popular sport it is, of course, often claimed that the preparations are not used for muscle building or to increase performance, but for medical reasons." (StA 7)

However, the same public prosecutor limited his statements on the prohibition of self-doping later: spor

"Since there are so few cases, I cannot make a valid statement." (StA 7)

Indications that the (missing) medical indication was taken into account were only found in 25 procedural files, and even then those indications were mostly brief. In 13 cases, the accused defended themselves with reference to a medical indication, either by presenting a prescription or by making a general reference to an illness without presenting a medical prescription. Only in one case, in which also the accused's association had submitted various medical reports on the player and his illnesses, further investigations into the medical indication were made; the proceedings were ultimately closed for other reasons.

In the majority of the proceedings, the public prosecutor's offices did not examine this element in more detail. In four cases, for example, the lack of indication was obvious because the public prosecutor's office was investigating the use of cocaine. Cocaine in Germany is neither an authorised drug nor - according to the investigators - a component of authorised drugs. In two cases the accused admitted (from the outset or at the request of the investigators) that there was no medical indication. NADA's assessments of the lack of indication are found significantly more frequently in the files, such as the statement that in Germany "at present no drugs with chlorodehydromethyl testosterone as an active substance in human domain" are approved or that accidental ingestion by drugs is "almost completely excluded". Equally widespread are references by NADA to the absence or existence of a "Therapeutic Use Exemption" or a special permission. ${ }^{33}$

33 In case of certain clinical symptoms, NADA or the international sports governing bodies can grant a Therapeutic Use Exemption (TEU) for the use of prohibited substances or methods under the World Anti-Doping Code (WADC). 
bb) Intention of gaining an advantage in a competition of organised sport

The perpetrator has to participate in a "competition of organised sport" (section 3 (2)) or has to have the intention of gaining an advantage in such a competition caused by self-doping (section $3(1)$ ). In accordance with the legal definition in section 3 (3), a competition of organised sport is any sporting event

1. organised by, on behalf of or with the approval of a national or international sports organisation, and

2. which requires compliance with rules adopted by a national or international sports organisation which are binding upon its member organisations.

\section{(1) Interpretation of the characteristic "competition of organised sport"}

The element aims to exclude private tournaments from the scope of the prohibition, as self-doping in the leisure sector does not affect the integrity of organised sport to an extent that would justify criminal liability. ${ }^{34}$ According to the explanatory memorandum to the Act, the competitions covered are primarily sporting competitions of top-class sport and competitive sport, such as the Olympic and Paralympic Games or Youth Games, World Games, national or international championships, games or competitions of a national or international league, national or international cup competitions or international friendly games. Major running events (e.g. marathons) and regional leagues, sports festivals and sports events organised by private organisers are also covered, if and insofar they have been recognised by the responsible (national or international) sports organisations in advance. ${ }^{35}$ The competition has to be organised by a national or international sports organisation, i.e. a legal entity or an association of persons whose main task is to promote sporting activities. ${ }^{36}$ The sports organisation has to have a "certain degree of organisation"; in addition, solely local clubs are not covered.

Therefore, section 3 does, for example, not cover "sole company races, recreational football tournaments, solely private sporting activities (e.g.

34 BT-Drs. 18/4898, p. 27; Erbs/Kohlhaas/Wußler, 232 EL August 2020, AntiDopG \$3 Rn. 9.

35 BT Drs. 18/4898, p. 28.

36 Erbs/Kohlhaas/Wußler, 232 EL October 2020, AntiDopG $\$ 3$ Rn. 10. 
jogging in the park) or competitions which are held exclusively as part of school sports (e.g. games between different schools)". It is discussed in the legal literature whether competitive bodybuilding is subject to the definition of organised competition. ${ }^{37}$

In 21 cases, the files contained information on organised competition; in the remaining 82 cases, this element was not addressed. In nine cases, the assumption of an organised competition was based on a notification from the NADA, which carried out doping controls within the framework of competitions.

In one case, the public prosecutor's office asked the NADA whether a pre-season encounter already falls under the definition of a competition of organised sport in accordance with section 3 (3). The NADA considered the encounter as a competition by stating that although the matches were "basically part of the preparation before the actual season", they "did not have the character of friendly matches in which the focus is exclusively on the 'private sporting activities of the participants' or the fun of solely recreational activities. The aim of the pre-season matches is rather to prepare for the season in a targeted and immediate manner under 'almost competitive conditions'". The public prosecutor's office accepted the assessment of the NADA.

In four proceedings, matches were assumed to be competitions since the players concerned were Bundesliga (German football league) players.

In six cases, the public prosecutor's office carried out internet research itself and checked, for example, via the accused's Facebook page whether they took part in competitions. On this basis, it was expressly declared in one case, albeit without legal examination, that the participation in bodybuilding competitions is covered by the criminal offence. In all other proceedings against bodybuilders, the question of whether competitions in this area fall within the scope of section 3 was not discussed.

It was also mentioned in the interviews, that the interpretation of the concept of competition turns out to be a problem in the application of section 3 .

"There are certainly opportunities for disputes in organised sports events in, let's say, niche areas. I'm thinking of a bigger fight night, for example, which

37 MüKo-StGB/Freund, 3rd ed. 2018, AntiDopG $\$ \mathbb{S} 1-4$ Rn. 39. Against the applicability of section 3 to bodybuilding is argued that bodybuilding is not a sport but the exhibition of bodies; Brill, SpuRt 2015, 153, 154; in addition Samson-Baudisch, Der Missbrauch von anabol-androgenen Steroiden im freizeitorientierten Bodybuilding, 2014, p. 18. Differently BGH NStZ 2010, 170. 
is organised somewhere. Or you think of a city marathon, in a smaller city, where you have to look closely indeed." (StA 1)

"This definition is of course difficult. What is a sports organisation? Does a competition of bodybuilders, Mr. and Mrs. Universe for example, fall under the scope of the criminal offence? It's rather difficult. To the best of my knowledge, there is no sports organisation in the bodybuilding field." (StA 5)

The different approaches of the public prosecutor's offices appeared particularly in the area of bodybuilding. Whereas mostly no decision was made regarding the competitive character (and the proceedings were closed for other reasons, see below 3., c.), some public prosecutor's offices did not conduct proceedings in the area of bodybuilding because of selfdoping at all, but only because of section 2 .

"We don't even examine section 3 in these cases. Section 3 presupposes that one must gain an advantage in a competition of organised sport (...). That was already clarified before I started working here. The NADA has already said that. That doesn't matter to us. (...) We handle these proceedings under the framework of section 2. The question whether someone has participated in competitions or not can be considered as an aggravating factor in the proceeding. Section 2 leaves enough room for that." (StA 5)

\section{(2) Intention of gaining an advantage}

The accused is tested positive for cocaine during a general competition control on the occasion of a Bundesliga encounter. The athlete is a member of a general test pool of the NADA (ATP). The accused already confessed during the first interrogation by the police. He stated that he started using cocaine for private reasons but never used it to improve his performance in competition. He would be in therapy for some time because of his cocaine addiction. The public prosecutor's office considers the guilt to be minor and closes the proceeding in accordance with section 153 StPO.

A doping substance has to be taken with the intention of gaining an advantage in a competition of organised sport (section 3 (1) and (2)). While in most cases the intention of gaining an advantage is already indicated by the nature of the drug, especially when taking stimulants other motives -which are partially understandable - can also come into consideration. In 
several proceedings, the accused denied having taken the substances to obtain a sporting advantage.

In the proceeding outlined at the beginning, the public prosecutor's office did not further verify the information provided by the accused, but closed the proceeding in accordance with section $153 \mathrm{StPO}$ due to minor guilt. Public prosecutors also reported in the interviews that an intention of gaining an advantage is denied by the accused. However, corresponding statements were predominantly evaluated as self-serving declarations.

"What can be a problem is whether these substances have been used for doping purposes in sport. The statement that the intake of the substance would not have been for doping purposes in sport, but to increase potency or to relieve pain, is not rare. That is something that is arguable. From our point of view, these statements are usually self-serving declarations, attempts to get out of criminal liability. But these aspects are disputed." (StA 4)

However, taking a substance or applying a method only becomes "doping" when it is actually used to improve performance. The intention of gaining an advantage as an element can therefore serve as a corrective in order to limit the offence to actual doping relevant violations. In one trial, an athlete was accused of having given himself an infusion of at least 100 millilitres of liquid for "liquid compensation after weight making" for a wrestling competition. A summary penalty order was issued against the accused, but he was acquitted in the main trial, because it could not be proven that he has had an intention of gaining an advantage. The court concluded that "the accused would not have gained any advantage for the competition [...] if he had been administered liquid by infusion [...] two days before the competition and at least one day before the weighing. Thus, it remains questionable, after the taking of the evidence, where the advantage [...] of an infusion of more than $100 \mathrm{ml}$ is for the accused. This advantage is a legal prerequisite.

\section{(3) Interim result}

The study shows that the element of organised competition has not yet taken counters in practice. The existence of an organised competition is almost without exception not subject to substantive examination. However, examining the relationship to a sporting competition would be necessary, especially in the field of bodybuilding. 


\section{B. Results of the evaluation}

Especially when using illegal drugs, it can be questionable if the athlete has an intention of gaining an advantage. Substances such as cocaine have a psychologically and physically performance-enhancing effect and can therefore be used immediately before a competition to improve performance. At the same time, cocaine is a widespread "party drug" whose private use is not implausible. If the accused denies it a clear proof of the intention of gaining an advantage is hardly possible.

c) Restrictions of criminal liability in section 4 (7)

Section 4 (7) restricts the group of possible perpetrators for reasons of proportionality. This restriction is also based on the assumption that manipulation through doping only leads to a "loss of confidence in the sports system and relevant damage" if the perpetrators are "outstanding sportsmen and women" who "practice their sport performance-oriented and competition-oriented at a high level or who gain considerable income from their sporting activities". 38

Therefore, only persons who are either top athletes in organised sport (section 4 (7) no. 1) or who directly or indirectly gain substantial income from their sporting activities (section 4 (7) no. 2) can be liable to prosecution. ${ }^{39}$

aa) Athletes included in a Registered Testing Pool

A person who is as a member of a Registered Testing Pool subject to Outof-Competition Testing within the doping control system is considered as top athlete. $11.7 \%$ of the accused were members of a Registered Testing Pool of the NADA, $74.8 \%$ were not included in such a Testing Pool and in $13.6 \%$ of the proceedings no information was provided. In all relevant cases, it was already stated in the NADA notification whether an athlete belonged to a Registered Testing Pool: "[The accused] falls within the scope of application of the sanction regulations of section 4 (7) no. 1 AntiDopG.

38 BT-Drs. 18/4898, p. 31.

39 Some argue that the concept of top athletes includes both test pool athletes and athletes with significant revenue, see for example Erbs/Kohlhaas/Wußler, 228 EL January 2020, AntiDopG $\ 4$ Rn. 5. Ultimately, the conceptual classification is not important. 
He or she is a member of the NADA Testing Pool within the doping control system and is subject to Out-of-Competition Testing.

One of the public prosecutors interviewed criticised that the investigating authorities were not able to check independently whether the accused was a member of a Registered Testing Pool."

"If being member of a Registered Testing Pool is a prerequisite for being a perpetrator, law enforcement authorities should be able to check exactly: Who is in this Testing Pool? There has to be the possibility of access for law enforcement authorities (...). If the criminal prosecutor cannot independently check whether a certain person is a member of a Registered Testing Pool, he ultimately cannot pursue his or her criminal liability."

bb) Significant revenue

(1) Teleological appropriateness of the restriction of criminal liability

Not only athletes who are subject to Out-of-Competition and In-Competition Testing can be exposed to a special incentive for the usage of prohibited doping substances or doping methods. Rather, such an incentive also exists when significant revenues are gained through the sport. In the view of the legislator, the penal provisions of self-doping (section $4(1)$ no. 4,5 ) as well as the purchase and possession of doping substances for the purpose of gaining an advantage in competition (section 4 (2) in conjunction with section $3(4))$ should therefore also cover athletes who directly or indirectly generate significant revenue from their sporting activities. The legislator also justifies the restriction of criminal liability by stating that these athletes are also role models and claim the trust that "they have achieved their sporting successes by pure means". ${ }^{40}$ The explanatory memorandum to the Act cites organised motor sports, professional boxing and the 3rd men's football league as examples. In these sporting activities, the athletes are typically in the public eye with their sporting achievements and could therefore undermine the integrity of organised sport as well.

40 On this and on the following BT-Drs. $18 / 4898$, p. 31 et seq. 
This restriction of criminal liability is described in the legal literature as arbitrary and difficult to reconcile with the purpose of the law, ${ }^{41}$ but is accepted in practice as the result of an exercise of discretion by the legislator:

"Sometimes this is difficult to determine, but I think it is intended to be tailored to this circle. It is a political question who you want to include. This may be difficult to determine; this is a practical question that also arises elsewhere." (StA 4)

\section{(2) Difficulties of application}

Voices in the legal literature criticise the concept of "significant revenues" for its insufficient certainty as well. ${ }^{42}$ In fact, the wording of the law causes not inconsiderable problems in its application. When asked what the greatest legal difficulties in providing evidence in self-doping cases are, a public prosecutor replied:

"In the area of top-class sport it is certainly section 4 (7), if we do not have an atblete who is included in a Registered Testing Pool. (...) To provide the proof of revenue of a certain significance is often difficult. That one has to say, perhaps the limit is reached and it is not possible to affirm the significant revenue is certainly difficult (...)." (StA 1)

In the case files evaluated, only a few accused practiced a sport at a level that is typically of interest to a wider public (one professional boxer and one kick boxer who had previously won international titles, as well as one Bundesliga wrestler and one Bundesliga weightlifter). More often, investigations were directed against less successful athletes (kick boxers, triathletes, bodybuilders). In the case of an arm wrestler, a witness testified:

"I cannot imagine [that the accused gains significant revenue] either, since money does not play an important role in our sporting activity." (extract from file)

Proceedings involving investigations against athletes with significant revenue are quantitatively underrepresented. Even though in nearly half of the relevant procedural files $(48.5 \%)$ it was suspected that the athletes gen-

41 See for the whole issue MüKo-StGB/Freund, 3rd edition 2018, AntiDopG $\mathbb{\$} \mathbb{1 - 4}$ Rn. 112 et seq.

42 Eising, Die Strafbarkeit des Eigendopings, 2018, p. 210, who demands clarification by the legislator. 
erated such revenue, this revenue was in only $12.6 \%$ of the cases actually ascertainable; ${ }^{43}$ and that although public prosecutor's offices base their work on a low-threshold understanding of "significant revenue".

\section{(3) Definition and determinability of the formulation}

The explanatory memorandum to the Act mentions as a prerequisite "the repeated attainment of economic advantages"; these have to be "significant benefits" that go far beyond the mere reimbursement of costs. ${ }^{44}$ The legal literature favours a case-by-case consideration ${ }^{45}$ or a sport-specific application, according to which an athlete who practices a cost-intensive sport should also be able to gain greater revenue. ${ }^{46}$ Such a case-by-case interpretation could not be determined in the evaluation. Rather, it is apparent from several procedural documents that the investigating authorities asked whether revenue was generated in an amount subject to social insurance contributions ("450 Euro"). In the interviews, two public prosecutors also confirmed that this amount serves as an indication for them. Another public prosecutor combines revenue and presumed expenses and then operates with an amount "in the range of 900-1000 € (...) per month" (StA 3).

However, there is also uncertainty about the adequacy of comparatively low revenue limits:

"I had a problem the other day with the question of what constitutes significant revenue. The [accused] played American football, earned 1,000 $€$ a month, but of course he also received board and lodging. So I asked myself: Does an American football player who plays in the 1st Bundesliga (German National League) and only earns 1,000 € per month has a significant revenue, yes or no? As far as I have seen, there was also no existing case-law on

43 Nevertheless, the vast majority of these proceedings ended with terminations, since there were no sufficient grounds for suspicion in other respects, the guilt was minor or the accused had left the country and a request for legal assistance was unlikely to succeed due to the lack of criminal liability in the country of residence.

44 BT-Drs. 18/4898, p. 31 et seq.

45 Erbs/Kohlhaas/Wußler, 228 EL January 2020, AntiDopG $\$ 4$ Rn. 8.

46 Lehner/Nolte/Putzke/Putzke, Anti-Doping-Gesetz, $\mathbb{4} 4$ Rn. 33, who draws the line from average salary payments of (men's) football players in the 3rd league and the regional leagues for annual net income of 18,000 Euro or three times payments of 1,500 Euro. 
this. Therefore, it would be interesting if the legislator could specify that in greater detail." (StA 1)

Other respondents also expressed the wish that the legislator or the Supreme Court decisions provide conceptual clarity.

"That perhaps would be an idea for the AntiDopG, to think about whether the range should somehow be predefined. That would be desirable. Or perhaps the design can be left to the dispensation of justice (...)." (StA 3)

"Yes, I think that overall it [the AntiDopG] is successful. The cases in topclass sport are still limited, if I understood that correctly, but I do believe that it is successful overall. (...) What is important for us (...) is the specification of the revenue." (A 2)

The courts of first instance do not seem to have had any experience with this element so far:

"No, we have not applied that yet. I do not know. The term is chosen poorly because it may introduce a new category in criminal law regarding revenue. At least I do not know of any criminal offence immediately in which that criterion appears as well." ( $R$ 1)

A representative of sports within an association $(D)$ still considers the wording to be "too vague". It is therefore not surprising that the lowthreshold above which public prosecutor's offices presume significant revenue does not appear to be present in the awareness of athletes as yet.

"To be honest, as I understood it, this can onlyaffect athletes in the national squad, including the winning sports, i.e. national teams, national squadplus league operations: football, basketball, handball. I find it difficult to quantify this. (...) I find a number difficult. I think, one should rather say: on a professional, international level in the national squad or national leagues. The Bundesliga is, I think, always the limit as to whether someone is a professional player or not and can therefore live on his salary. Perhaps this is where I would quantify that it is a living." (A 1)

Another athlete representative pleaded for a significantly higher threshold:

"One criterion for the promotion of sports aid is, for example, 45,000 € per year. This is (...) taken as a criterion for whether someone should receive support or not. I think that would be helpful as a first orientation." (A 2) 


\section{(4) Investigations}

Search warrants and confiscation orders are usually based on the assumption that the accused would obtain significant revenues. This assumption is made without further substantiation. For example, in proceedings conducted against a bodybuilder the public prosecutor's office solely stated:

"She finished fourth in the women's physique category. She therefore earns not inconsiderable revenue from her sporting activities." (Excerpt from file)

The investigation file, however, does not contain any further details on the amount of revenue. Occasionally, internet research on the accused, such as research regarding possible employment and possible sponsors, was conducted in advance. Criminalistic experience also seems to play an important role. For example, in proceedings against strength athletes, investigations for a violation of section 3 are often not even conducted, because many public prosecutors assume that strength athletes are neither top athletes nor generate significant revenue.

"That are experiences that one acquires over the years, i.e., power lifters and bench pressers, stone lifters or arm-wrestlers and whatever they are called: if one carries out investigations in that area [...it becomes clear] that all of them live in orderly and stable conditions, but not in conditions that offer points of attack that significant revenue is gained (...)." (StA 3)

Also, the file evaluation showed that the investigations against power athletes are focused quickly on violations of section 2 .

"A power lifter who is reported by the NADA, can usually be convicted, but not in accordance with section 3 but in accordance with section 2, because he usually possesses significant quantities of doping substances." (StA 3)

In the further course of the investigations, mostly simple investigation measures are carried out. These include the evaluation of confiscated account statements or employment or sponsorship contracts as well as the questioning of the accused and witnesses. More elaborate financial investigations through involving banks are rare. They were conducted when the case gave particular cause: In one case, this seemed to be the (additional) reference to violations of the German Narcotics Act (BtMG); in other cases, it seemed to be the investigators' criminalistic sense that the accused had made significant revenue and that the other elements were also fulfilled. 


\section{B. Results of the evaluation}

In an exemplary case in which the public prosecutor's office conducted a broad investigation and also carried out financial investigations at an early stage, the NADA had initially reported a positive doping sample in the margins of the German championships in kickboxing. The preliminary investigations were initially conducted on the grounds of section 4 (1) nos. 3, 2 (3), since the accused was not an athlete included in a Registered Testing Pool and it was at least not obvious that significant revenue was gained. On this basis a search and - rather unusual - also a blood sample (section 81a StPO) were ordered. Although the blood sample was not necessary for the proof of criminal liability for possession, it was legally permissible and would above all have facilitated the proof of self-doping, in the event that the accused gained significant revenue and thus would fall within the scope of section 4 (1) nos. 4,5 . In order to examine this, the public prosecutor's office determined what revenue the accused had earned by requesting banks. Since the accused's revenue could ultimately not be determined, the order of punishment was finally issued in accordance with section 4 (1) nos. 3, 2 (3). However, such an effort has only been made in a small number of proceedings.

\section{(5) Summary}

In summary, this element causes practical problems, despite or rather because of a rather low threshold. In many cases, it does not seem to be clear to the athletes that even revenue in an amount that is subject to social security obligation $(450 €)$ can give rise to a pursuant initial suspicion. Practical difficulties were also expressly pointed out or at least hinted by criminal prosecutors; several respondents expressly wished for clarification by the legislative or the Supreme Court. It is particularly clear that the low threshold widely used by the public prosecutors' offices leads to investigations due to violations of the self-doping ban even against athletes, who gain only little revenue and are not in the public eye. The lesser injustice of such acts and the resulting disproportion to further investigations is considered by the public prosecutor's offices (as in other cases of minor delinquency) by closing the proceedings.

Especially investigations against athletes with revenue on the borderline of significance can be elaborate for public prosecutor's offices: Smaller sums are not only per se less conspicuous than larger amounts. These payments are also not always visible as incoming payments to an account. Above all, the importance of smaller expenses for the practice of sport in- 
creases the lower limit for gross income set. In other words: The lower the net value that causes the assumption of "significant revenue", the greater the importance of the expenses and the investigations concerning them.

cc) General assessment of section 4 (7)

\section{(1) Classification}

In the legal literature, the question as to whether section 4 (7) is an element or a solely objective condition of criminal liability is controversial. ${ }^{47}$ The systematic position of the provision in section 4 and not in section 3 argues for the assumption of a solely objective condition of criminal liability. It indicates that by limiting the circle of addressees the legislature intended to carry out an objective restriction of criminal liability. 48

However, one of the investigated proceedings was closed by the public prosecutor's office in accordance with section 170 (2) StPO on the grounds that it was "not possible to determine with certainty" whether the accused had "taken the doping substances at a time when he was already aware of his inclusion in the Registered Testing Pool and thus of his norm addressee status".

Considering the systematic position of the group of perpetrators, it was criticised that such an essential prerequisite of criminal liability should not be included as late as in the seventh subsection of the penal provision.

"There are of course many laws that are even more complex and complicated in structure. Nevertheless, from a legal point of view, from the point of view of the user and, above all, the addressee, a clearer accentuation, highlighting or the placing at the beginning of the respective circle of addressees and the respective circle of criminal law would be desirable." $(N)$

47 See Erbs/Kohlhaas/Wußler, 228 EL January 2020, AntiDopG $₫ 4$ Rn. 11; MüKoStGB/Freund, 3rd ed. 2018, AntiDopG \$S 1-4 Rn. 113.

48 This is also the terminology used in the explanatory memorandum to the Act, BT-Drs. 18/4898, p. 31. Critical with reference to the obligation to inform about the affiliation of an athlete to the Registered Testing Pool Erbs/Kohlhaas/Wußler, 228 EL January 2020, AntiDopG $\$ 4$ Rn. 11. 
(2) Reason for low case numbers and rare indictments

The restriction of the circle of addressees in section 4 (7) was considered to be the main reason for the low number of cases in the field of self-doping.

"The regulation of section 4 (7) certainly is one reason why we have only very few proceedings in the area of top-class sport. One must be aware that the number of athletes who either fall into the Registered Testing Pool or actually receive significant revenue from their sporting activities is not very large." (StA 1)

This impression was confirmed by the file investigation; accused were often not athletes included in a Registered Testing Pool and the purchase of significant revenue could also not be proven. A reduction of the obstacles formulated in section 4 (7) could cause more proceedings being brought to charge.

"It often becomes difficult to provide proof as soon as you don't have an athlete included in a Registered Testing Pool. If you wanted to have more cases in this respect, a readjustment would certainly be useful." (StA 1)

\section{(3) Unequal treatment of athletes}

The restriction in section 4 (7) was justified by stating that the integrity of organised sport is threatened solely by the behaviour "of competitive athletes perceived by the public; purely recreational athletes should therefore not be covered by the criminal provision, even if they participate in competitions of organised sport (e.g. major running events). ${ }^{49}$

In the legal literature, section 4 (7) has been criticized as an "arbitrary" restriction of the offence, which has "no objective connection with the wrongfulness of the doping offences committed". ${ }^{50}$ The NADA expert expressed similar criticism in the interview.

"In our exchanges with the public prosecutors' offices we have seen that we are now putting the athletes included in a Registered Testing Pool and the ones who clearly generate an income from the sporting activity in a situation that puts them in a negative privileged position compared to all other athle-

49 BT-Drs. 18/4898, p. 32.

50 MüKo-StGB/Freund, 3rd ed. 2018, AntiDopG \$S 1-4 Rn. 112. 
tes who may be taking part in the same event or competition. This should be brought back into focus, if necessary." $(N)$

Indeed, section 4 (7) has the consequence that athletes who participate in the same competition are treated differently by the AntiDopG. The distinction between competitive and recreational athletes described in the explanatory memorandum to the Act may make sense in certain competitions, such as an urban marathon, which is also open to popular sport. However, the restriction in section 4 (7) extends well beyond these cases. For example, in a German championship, usually only competitive athletes participate; athletes included in a Registered Testing Pool, however, are only those who have already made the leap into the corresponding squad (depending on the sport: A squad (including athletes competing at an international level) or B squad (including athletes who are likely to compete at an international level in the near future). Although the athletes compete directly with each other, only those who already belong to the squad should be punished - whereas, those who try to be accepted into the squad through their participation should not be punished. ${ }^{51}$

The unequal treatment of participants in the same sporting competition is difficult to reconcile with the aims of integrity and fairness of organised sport, especially in the case of events in which recreational athletes usually do not participate. Much evidence suggests that the offence should not be restricted by personal characteristics, but rather by clearly limiting the competitions covered (see C II 1).

\section{d) Active repentance}

\section{aa) Legislative context}

Pursuant to section 4 (8), anyone who voluntarily relinquishes the actual power of disposition of the doping substance before using it or allowing it to be used will not be punished for the purchase or possession of doping substances pursuant to section 3 (4). The provision was not contained in

51 See also Freund, who describes the situation as follows: "Of two athletes who have doped themselves in advance and therefore participate in the same competition in violation of sports law, only the one who has perhaps only just moved up into the circle of 'top athletes' is punished, while his equally doped competitor goes unpunished if he is only very close to being accepted into this 'illustrious' circle". See MüKo-StGB/Freund, 3rd ed. 2018, AntiDopG $\$ S \mathbb{1 - 4}$ Rn. 112. 


\section{B. Results of the evaluation}

the government draft, but was introduced into the law by a recommendation of the Sports Committee..$^{52}$ As a personal reason for suspension of sentence, the provision is intended to enable impunity if the athlete distances him- or herself from self-doping and voluntarily ensures that he or she can no longer harm the integrity of sport by using the doping substance. Prerequisite for impunity is that the athlete relinquishes the power of disposition of the doping substance before using it. ${ }^{53}$ This requires that the athlete performs an action that is visible to the outside world and results in the athlete no longer being able to dispose of the doping substance (disposal, handover to the authorities, etc.). ${ }^{54}$ In addition to this consideration regarding the protective purpose, there are also special preventive reasons for the waiving of punishment in such cases: In cases in which the athlete apparently refrains from self-doping intentions by giving up the possession, it is not necessary that the penalty impinges on the athlete. Consequently, the reason for suspension of sentence further presupposes that the surrender of actual power of disposition is voluntary. The athlete must give up the power of disposition of the doping substances for self-imposed motives and voluntarily ensure that he or she can no longer harm the integrity of the sport with the doping substance. ${ }^{55}$

The creation of a reason for suspension of sentence in case of active repentance was welcomed by the legal literature. ${ }^{56}$

\section{bb) Results of the study}

In the files evaluated, the provision did not play a role. In particular, no case was ascertainable in which criminal proceedings were (partially) discontinued because in the course of the investigation it had turned out that an athlete had relinquished the power of disposition of doping substances which he or she allegedly had purchased or possessed before the investigations began. However, whether and to what extent the provision has individual significance for athletes who dispose of doping substances and re-

52 Cf. on this and the following BT-Drs. 18/6677, p. 4, $12 \mathrm{f}$.

53 Lehner/Nolte/Putzke/Putzke, Anti-Doping-Gesetz, $\$ 4$ Rn. 94.

54 Lehner/Nolte/Putzke/Putzke, Anti-Doping-Gesetz, $\$ 4$ Rn. 94.

55 Lehner/Nolte/Putzke/Putzke, Anti-Doping-Gesetz, $\$ 4$ Rn. 95.

56 MüKo-StGB/Freund, 3rd ed. 2018, AntiDopG $\mathbb{S S ~ 1 - 4 ~ R n . ~ 7 5 ; ~ f u r t h e r m o r e ~ L e h n e r / ~}$ Nolte/Putzke/Putzke, Anti-Doping-Gesetz, $\$ 4$ Rn. 97. 
frain from doping intentions with a view to the criminal privilege cannot be determined.

From the low practical significance of the provision on active repentance, however, it cannot be deduced, that the reason for suspension of sentence does not have justification relating to criminal policy. On the contrary, it is conceivable that indications (e.g. statements from the environment, documents) at first suggest the initial suspicion of the purchase or possession of doping substances, but as a result of the investigations it turns out that the athlete has voluntarily relinquished the power of disposition in the meantime. The continuation of the investigations or even a punishment is not necessary in such cases because of the considerations regarding the protective purpose and special preventive reasons mentioned under aa) above. Apart from that, this reason for suspension of sentence can also be seen as a certain corrective to the comprehensive, not quantitatively limited criminal liability for the purchase and possession in accordance with section 4 (2).

\section{Findings on procedural issues}

a) Knowledge obtainment

aa) Ways of obtaining knowledge

The law enforcement authorities learned about possible cases of self-doping in particular through reports or informational notifications from the NADA (65.0\%). The NADA based its reports primarily on the result of a positive doping sample (56 proceedings) or on anonymous information through its BKMS reporting system (Business Keeper Monitoring System) (9 proceedings). ${ }^{57}$ Following a report by the NADA, the public prosecutor's offices usually initiated preliminary investigations within a few days. The importance of the NADA for obtaining knowledge of crimes in the area of self-doping was also confirmed in the interviews.

"Since in the field of top-class sport the NADA is practically the only source of possible reports, the information is relevant. We receive 20 to 30 reports by the NADA per year. Otherwise there are no initiations for any investiga-

57 Two further proceedings were initiated by the NADA itself due to media reporting or public statements by the athlete. 
tions in this area. That is why the cooperation with the NADA is very important." (StA 3)

In $25.2 \%$ of the cases, the initial suspicion of the public prosecutor's offices arose from other proceedings, in particular from investigations against dealers of doping substances in accordance with section $2 .{ }^{58}$ In those cases the law enforcement authorities initiated proceedings against the customers of the substances, whose data were taken from the dealers' order lists. The practice among the public prosecutor's offices was inconsistent: While some investigated solely on the basis of section 2 (purchase), ${ }^{59}$ other public prosecutor's offices conducted the proceedings - even without any indications of participation in sports competitions or the capacity as a perpetrator in accordance with section 4 (7) - on the grounds of selfdoping. However, all of those proceedings were closed without conditions.

Five of the proceedings were reported to the public prosecutor's offices by customs, two proceedings by third party complainants. There were no indications from sports associations in the sample investigated.

Fig. 3: Origin of the initial suspicion

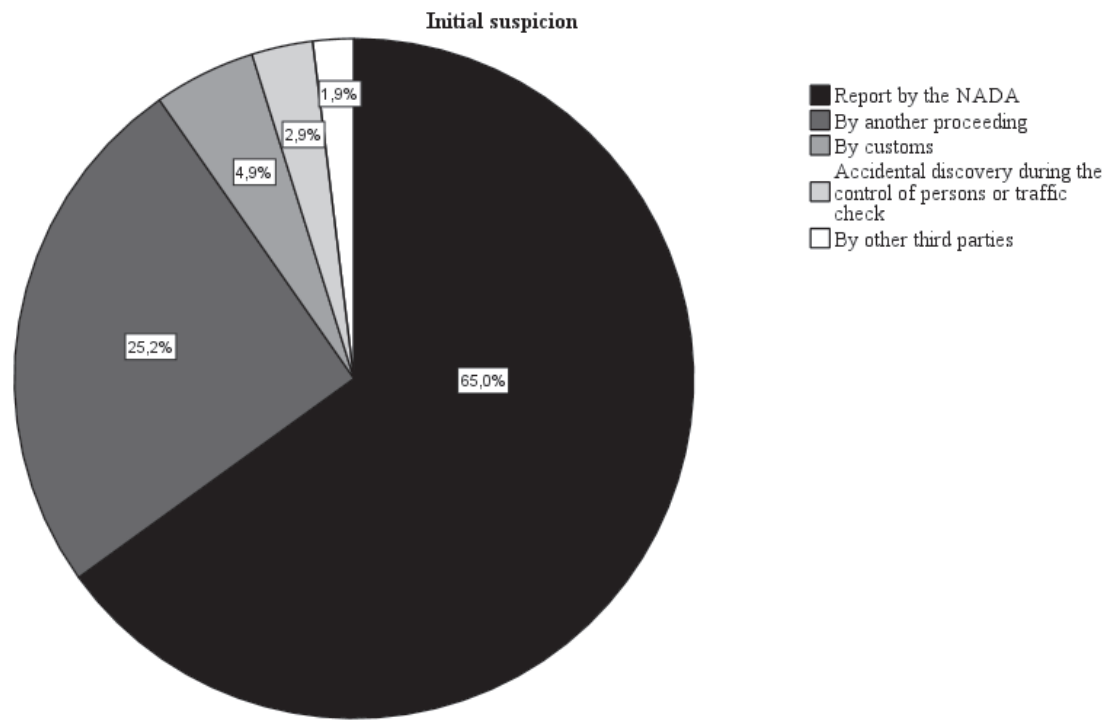

58 Chance finds during house searches or telecommunications surveillance also played a role.

59 These proceedings were then not included in the local evaluation. 
bb) Leniency

In connection with acquiring knowledge about cases of self-doping, the interviewees regularly made the introduction of a leniency subject of discussion. A leniency for athletes, who are accused of self-doping, does not yet exist. Section 46b I 1 of the German Criminal Code (StGB) in conjunction with section 100a (2) no. 3 StPO refers solely to the qualifying offence of committing the crime commercially or as a member of a gang in section 4 (4) no. 2 lit. b and thus excludes the doped athlete from the privilege of a leniency. ${ }^{60}$

In view of the limited possibilities of obtaining knowledge, some of the public prosecutors interviewed argued in favour of extending the leniency to the area covered by section 3 . The prospect of mitigation of punishment or exemption from punishment could help to uncover structures and convict other doping athletes.

"At the top of the wish list would be, of course, to include a leniency in the AntiDopG because, as I said before, especially in top-class sport we don't get any cases. I already explained earlier how cases come up in mass sports and in weight training. Since practically all of these possibilities do not exist in top-class sport we do not get any cases in that area. Reports come either from the NADA or from the scene. [...] One would have to give the athletes an incentive to make a statement to the prosecution authorities. Especially since the dealer or the doctor would have the possibility to get a mitigation of the range of sentences through the general leniency. The athlete simply does not have that possibility. In my opinion, it is a very important signal to the scene to say cooperation is worth it." (StA 3)

On the introduction of a leniency for doping athletes, see in detail below (4.b.aa.).

b) Investigation measures

In $54.4 \%$ of the proceedings, no investigations carried out by the prosecution authorities were documented in the files that went beyond the hearing of the accused. The investigation measures carried out often involved searches of flats, motor vehicles and business premises (38.8\%); urine,

60 On this in detail and critically: Cherkeh, SpuRt 2019, pp. 167, 168; Hauptmann/ Klarmann, SpuRt 2019, p. 197. 


\section{B. Results of the evaluation}

blood or hair analyses were less frequent $(6.8 \%)$ and an observation and a financial investigation were each only carried out in one case. Below the threshold of State intervention measures, online searches were often carried out to obtain an impression of the sporting activities and potential income of the accused.

"Often search measures are carried out, in both popular and top-class sport. The accused athlete's home and partly also the premises of his or her sports association are searched." (StA 1)

"The first measure is, of course, a search of the house. We actually always find something there with great success, if there's an initial suspicion." (StA 2)

Considerable differences between the public prosecutor's offices appear again in their willingness to carry out investigative measures.

In some cases, investigation measures were ordered at a very early stage of the proceedings. The legal prerequisites for the criminal liability of selfdoping - such as participation in a competition of organised sport or the capacity as a perpetrator pursuant to section 4 (7) - were not examined. Extensive - and for the accused invasive - searches of flats were often carried out; however, the proceedings were then closed with a view to the lack of the legal requirements of section 3, even when substances were found. In such cases a reverse procedure would be appropriate, both for reasons of effectiveness and with a view to the protection of the accused.

However, the reverse picture also emerged. Despite existing indications that doping substances were being taken proceedings were closed without carrying out investigations by stating that the legal requirements would not be met. An actual examination of the prerequisites of the offence, however, was not carried out.

The NADA reported a dart player who tested positive for amphetamines at the German Darts Masters. It was not an athlete included in a Registered Testing Pool. The public prosecutor's office waived further investigations. The proceedings were closed in accordance with section 170 (2) $\mathrm{StPO}$ on the grounds that there was no indication of significant revenue. However, possible income of the athlete was not examined by the public prosecutor's office.

In other proceedings, the questioning of accused persons was waived "because, based on experiences from other preliminary investigations, it was to be expected that the accused persons will make use of their right to si- 
lence. The proceedings are therefore to be closed pursuant to section 170 (2) StPO”. (Excerpt from file)

\section{c) Completion of proceedings}

Six of the investigated proceedings were ultimately not penalised as selfdoping but as violations of section 2 or closed subject to conditions in accordance with section $153 \mathrm{a}$ StPO.${ }^{61}$ In three cases a summary penalty order (of 30, 40 or 120 daily rates) was issued for the accusation of self-doping. ${ }^{62}$ The remaining proceedings regarding self-doping were closed; only four of these proceedings were closed with the imposition of a fine in accordance with section 153a StPO. No convictions for self-doping were found in the examined sample.

61 Since in these proceedings, the accusation of self-doping was raised, they were included in the sample (see p. 4). The outcomes were two convictions, two summary penalty orders and two terminations subject to conditions in accordance with 153a StPO for violation of section 2.

62 In one of the two summary penalty orders section 4 (1) no. 4 and 5 are named, but the explanatory memorandum solely refers to section 2 (3). 
Fig. 4: Completion of criminal proceedings
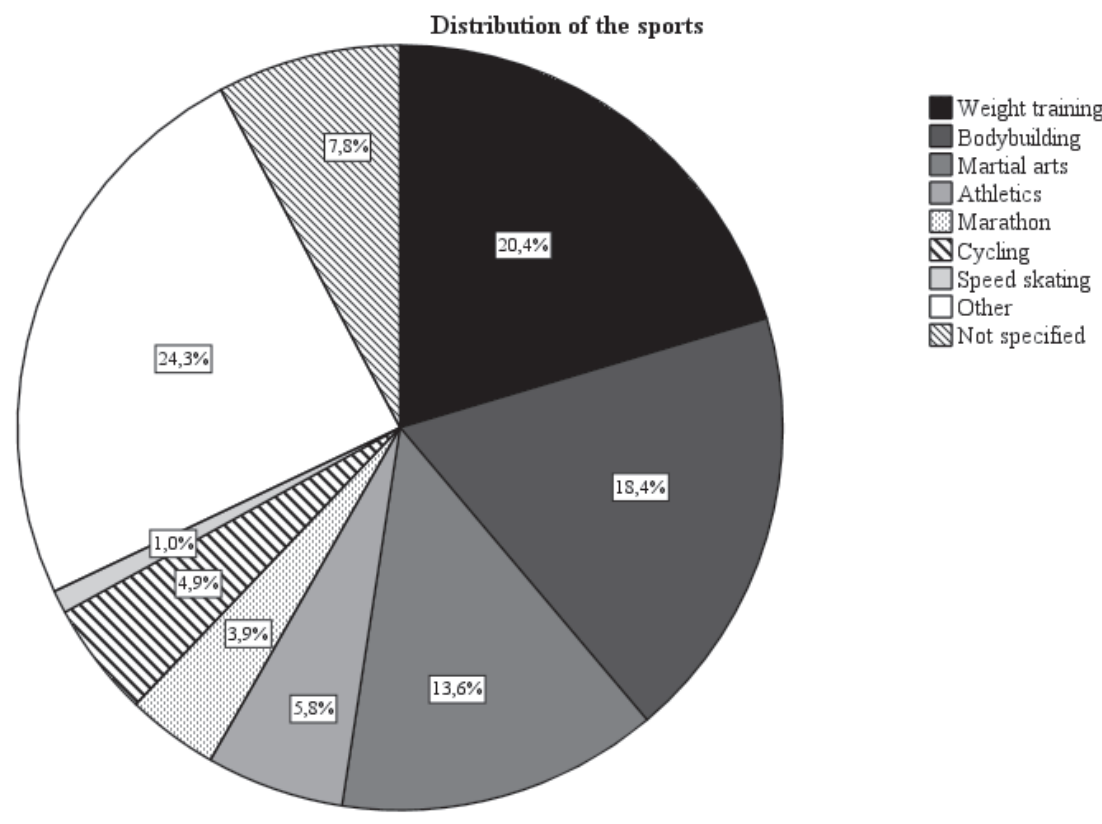

Also, the interviews confirmed that cases of self-doping have hardly ever reached the courts so far.

R 2: "I would say there wasn't a single case [of self-doping]."

Interviewer: "So this is not an offence that occupies you in practice, where we could now talk about the interpretation of the individual elements?"

R 2: "No, not at all."

aa) No convictions for self-doping

The evaluation of the procedural files provided by the public prosecutor's offices shows a remarkable conspicuousness: The proceedings that were conducted for a violation of the self-doping ban were almost without exception closed in accordance with section 170 (2), section 153 StPO.

The findings of the file evaluation coincide with the results of the interviews to the extent that the interlocutors confirm that so far there have 
been no convictions for violations of the self-doping ban after a main hearing. Indications of such convictions, which can be drawn from the provisional data for 2017 and 2018 provided by the Federal Statistical Office, cannot be confirmed by the public prosecutors interviewed. Instead, one public prosecutor told a specialised public prosecutor's office about two judgements allegedly delivered in his area of responsibility:

"I believe there was an error in the statistical recording." (StA 3)

A public prosecutor from another specialised public prosecutor's office stated on request about a judgment that might have been delivered in his area of responsibility:

"I currently do not recall any proceedings by the specialised public prosecutor's office that have led to a conviction for self-doping after the main trial was held." (StA 1)

One judge replied to the question whether he had encountered legal problems relating to the application of the offences related to self-doping:

"No, because I never used them." ( $R$ 1)

bb) Terminations in accordance with section 170 (2) StPO or section 153 $\mathrm{StPO}$

Whether proceedings were closed in accordance with section 170 (2) StPO or in accordance with section $153 \mathrm{StPO}$ depended less on the reasons for termination than on the practice of the respective public prosecutor's offices. ${ }^{63}$ In particular, proceedings were often closed in accordance with section 153 StPO even if the results of the investigation showed that the legal prerequisites were not met or could not be proven. In the explanatory memorandum to the writ of nolle prosequi doubts whether the legal prerequisites were met were not addressed, but the lack of previous convictions and the special preventive effect of the preliminary investigations were pointed out.

63 Some public prosecutor's offices did not close proceedings in accordance with section 170 (2) StPO in principle, while others chose either section 153 or section 170 (2) StPO without any identifiable differentiation criterion; still others did not close proceedings in accordance with section $153 \mathrm{StPO}$ if an element was demonstrably not fulfilled. 
"The accused has not yet made a criminal appearance. It can be expected that the accused has been sufficiently impressed and warned by the preliminary investigations carried out so far." 64

The analysis revealed various reasons for the termination of proceedings relating to self-doping, such as:

- The substance found was not a prohibited doping substance.

- The accused is domiciled abroad and a letters rogatory was not considered promising.

- There has already been "a considerable sanction on the part of the sports association" ${ }^{\prime}$, which makes criminal proceedings no longer appear necessary. ${ }^{66}$

- The proceedings lasted longer than one year and were closed "taking into account the further lapse of time that had occurred in the meantime".

In almost all proceedings it was difficult to prove all prerequisites for criminal liability.

In particular, the characteristic of "significant revenue" posed problems of proof. The public prosecutor's offices often did not carry out investigations in this respect, but decided to close the proceedings ("It cannot be assumed with a sufficient degree of certainty that the accused was generating revenue.").

Another reason for the termination of proceedings was the presentation of a medical certificate. A review of the medical certificate by the public prosecutor's offices was not apparent from the files; in some cases it was expressly pointed out that obtaining a "costly expert opinion" was "not proportionate to the importance of the matter". The proceedings were closed.

The experts would like to draw particular attention to two problems in the effective prosecution of self-doping: Termination because of the absence of intent regarding the use of doping substances (cc) and termination without apparent reasons $(\mathrm{dd})$.

64 The explanatory memorandums to the writs of nolle prosequis in accordance with section $153 \mathrm{StPO}$ read almost exactly the same at this point.

65 All following quotations are extracts from the files examined.

66 In these cases, the proceedings were closed in accordance with section 153 StPO. 
cc) Termination because of the absence of proof of intent regarding the use of doping substances

"The accused denies having committed the offence he or she is charged with. There are no direct witnesses. There is no evidence that would allow for a conviction without concerns. After carrying out the extensive investigations, it cannot be established with the probability required for an indictment that the accused has taken the substance knowingly and willingly.”

Despite the existence of a positive doping sample, public prosecutor's offices closed twelve proceedings on the grounds that it could not be proven that the accused took the doping substance knowingly.

(a) Terminations even took place if the accused's explanation for the substance in his or her blood or in his or her urine can only be described as far-fetched. For example, a racing cyclist explained his positive doping test in the accused's hearing as follows:

"In summary, these were tablets against hypertension and dehydration tablets for my mother who suffers from cancer. My mother wanted to put these tablets into a doser or dispenser. In this process a few tablets fell to the floor. I took one of the tablets from the dispenser and munched the others from the floor. (...) My mother explained only later to me what kind of tablets these are and what active substance they contain. That was Friday before the race."

The public prosecutor's office considered this testimony as sufficient for a termination in accordance with section 170 (2) StPO:

"The accused denied the charge brought against him and pleaded that he does not take 'such shit' and could not explain the proof of the substances amphetamine, heptaminol and hydrochlorothiazide in his blood. There is no other objective evidence available to convict the accused. In view of this factual situation and this evidence, bringing a public action has no chance of success. Because of the principle in criminal proceedings that in case of remaining doubts it always has to be decided in favour of the accused, it is not to be expected that the accused would be convicted."

(b) It has been repeatedly argued by accused that the proof of prohibited substances is due to contaminated food or drinking water. In the following, one proceeding is outlined as an example. 


\section{B. Results of the evaluation}

The accused was an athlete included in a Registered Testing Pool. He was a track cyclist. When the NADA carried out an unannounced training control, tamoxifen was detected in the athlete's urine. ${ }^{67}$ The NADA brought the matter to court. The accused denied having taken tamoxifen and gave an affidavit that he had never knowingly taken doping substances. He stated that the substance could at best have entered his body through contaminated drinking water and provided newspaper articles reporting that French drinking water was contaminated by tamoxifen. In addition, he provided evidence that he had been in a Belgian training camp shortly before the training control. The trainer had still kept the (partly opened) water bottles that the athletes used in the training camp. The water from these bottles was tested - an amount of tamoxifen that would have explained the positive doping test could not be detected.

The public prosecutor's office made the following summary statement:

"The conjecture of the accused that he unknowingly ingested the active substance tamoxifen via food supplements or contaminated water is in view of the chemical analysis carried out far-fetched. Concerning this matter, it also had to be considered that none of the other athletes in Team $\mathrm{X}$ tested positive for tamoxifen after their training camp in Belgium. Moreover, the NADA is not aware of any cases in which a doping case with the substance tamoxifen was caused by contaminated (drinking) water."

Nevertheless, the public prosecutor's office closed the proceedings in accordance with section 170 (2) StPO.

"Apart from the positive doping result, the criminal proceedings carried out did not provide any further evidence which would allow the conclusion that the accused knowingly applied or knowingly had applied the doping substance to him - or herself. The accused's testimony that he never knowingly or intentionally took the substance tamoxifen for a prohibited performance increase can therefore not be refuted with the certainty required for prosecution. In this respect, it had to be considered that in professional cycling it is not excluded that someone from the accused's environment administered the substance to the accused without his knowledge in order to promote his performance or to harm him. Therefore, the mens rea cannot be proven."

67 Tamoxifen is used for doping to counteract the side effects of anabolic steroids (e.g. breast growth in men). Since anabolic steroids are usually broken down more quickly than tamoxifen, however, often only this substance is detectable. 
The Office of the Attorney General comments on the termination of the proceedings as follows:

"The accurate report of the public prosecutor's office makes it clear that despite the extension of the criminal liability in the area of doping to so-called self-doping, it will remain difficult in individual cases to prove a crime in a way that would satisfy the Code of Criminal Procedure. In cases in which direct proof of the crime, for example, by perpetuation of evidence during searches or by testimonies from the accused's close environment, is not possible, failure of punishment despite positive blood samples will probably continue to happen frequently in the future."

(c) The public prosecutor's offices impose extraordinarily high requirements on the assumption of sufficient suspicion of a crime with regard to the mens rea. In the proceedings examined, the impression was created that the accused's testimonies were protection assertions. This applies not only to explanations apparently far-fetched - such as in case (a) - but also to blanket indications to contamination of consumed food, which are not confirmed by any investigations or practical experiences (on the contrary: in case (b) the claim that the doping substance would have been ingested through contaminated drinking water can be considered as refuted).

The practice observed would amount to the fact that proceedings for self-doping can in fact not be brought to trial ${ }^{68}$ as soon as the accused denies the act, even if a positive doping sample is present. This applies in particular if one follows the public prosecutor's office's argumentation in case (b), according to which the abstract possibility of manipulation by third parties should be sufficient to deny that the athlete knowingly took the doping substances by applying the principle in dubio pro reo.

That such a handling of the reference to contaminated food is by no means mandatory is shown by the conviction of the boxing athlete Felix Sturm by the Regional Court of Cologne for self-doping (for the self-doping in coincidence with the bodily injury caused thereby an imprisonment of six months was imposed). The 8th Criminal Division of the Regional Court of Cologne had initially rejected the opening of the proceedings on

68 Finding doping substances in the home of an athlete included in a Registered Testing Pool days or weeks after a test has been carried out does not seem very promising. The same applies to witnesses, especially since in these cases, taking the reasoning of the public prosecutor's office as a basis, the possibility of a will to incriminate could not be excluded. 


\section{B. Results of the evaluation}

the grounds that ${ }^{69}$ "based on the files and the expert opinions obtained, even at the end of a main hearing, the realistic possibility of an unintentional intake of the active substance remains. Therefore, a conviction for violations of the AntiDopG is not to be expected." ${ }^{70}$ However, the Higher Regional Court of Cologne annulled the decision and admitted the charges against Sturm to be brought before a different criminal division. The 12th Criminal Division of the Regional Court dealt in detail with the question whether the doping substance stanozolol ${ }^{71}$ could have been ingested by the athlete unknowingly. On the basis of two expert opinions, the chamber ruled out both the external influence hypothesis - it was argued that members of the opposing team had brought contaminated water bottles into the changing room after the fight - and the intake through contaminated food and food supplements. ${ }^{72}$ The importance of stanozolol as one of the "most popular competition steroids", which is "typically used in boxing", ${ }^{73}$ as well as the personal and professional circumstances of the athlete suggested the deliberate use of the doping substance. The press officer of the Regional Court rightly pointed out that in this case - as in other proceedings - the evidence had to and could be provided by circumstantial evidence:

"In such cases, we have a normal circumstantial evidence. And in this case, the court considered the accused's testimony, also based on the expert opinions, as a self-serving declaration."

The proceedings conducted in Cologne were complex - already due to the appointment of two experts and the comprehensive evaluation of their expert opinions. It therefore remains to be seen whether the verdict will in future cause public prosecutors' offices and courts - even in proceedings in-

69 Decision of the 8th Grand Criminal Division of the Regional Court of Cologne of 10 January 2019 - 108 KLs 17/18.

70 Quoted in accordance with the decision of the Higher Regional Court of Cologne of 04 April 2019 - 2 Ws 122/19. On this topic Lorenz/Bade, JR 2020, 324.

71 Stanozolol is an exogenous anabolic steroid which, when taken over a certain period of time, for example during preparation for a competition, supports the build-up of muscle mass with the aim of increasing maximum and explosive strength.

72 The expert opinions could prove that the substances had not been taken shortly after the competition; it was also proven that neither meat nor the food supplements used by the accused could have been contaminated with stanozolol.

73 Order of the Higher Regional Court of Cologne of 04 April 2019 - 2 Ws 122/19Rn. 28. See Kubiciel, SpuRt 2020, 206 seq. 
volving less prominent accused - to a more intense taking and consideration of evidence.

\section{dd) Termination without apparent reasons}

In many proceedings, terminations took place as soon as first difficulties in obtaining evidence arose. In some cases, however, it became clear that the investigating authorities had no will to prosecute. As an example, two proceedings will be outlined.

The accused - a marathon runner - is reported to the NADA via the anonymous whistleblower system. An evaluation of his Facebook profile by the public prosecutor's office shows that he repeatedly makes public statements about doping substances and also about their (personal) use. According to the anonymous informant, he had bragged to club members "at all times, whether appropriate or inappropriate," about consuming doping substances.

The public prosecutor's office initially decides not to open proceedings, because the information provided by an anonymous informant cannot be verified. The NADA criticizes the action of the public prosecutor's office and points out that the informant is willing to testify by name. Thereupon, the proceedings are initiated. However, they are closed shortly thereafter in accordance with section 170 (2) StPO. The public prosecutor's office justifies the termination on the grounds that it would be "one person's word against another' $s$ ". The NADA again contacts the public prosecutor's office and criticizes that the existing evidence would not have been sufficiently evaluated. Thereupon, the proceedings are reopened.

Two months later, the proceedings are closed again in accordance with section 170 (2) StPO; this time by pointing out that the athlete took part in competitions abroad and therefore German criminal law would not be applicable.

In the file there is no legal examination of the applicability of the AntiDopG. The result would not be justifiable anyway; the point of reference for the criminal act under section 3 (1) is the application of the substance and not the participation in a competition. For acts under section 3 (2), the public prosecutor's office should have examined the requirements of section 7 StGB. 


\section{B. Results of the evaluation}

One supplier was investigated for the dispatch of doping substances. Customs receive a list of customers and can intercept a package addressed to the accused. The package contains a large number of illegal substances. Customs forwards the find to the public prosecutor's office. In addition, the public prosecutor's office is informed that the receiver is a triathlete who takes part in national and international competitions and, as his homepage shows, is supported by various sponsors. The proceedings are closed by the public prosecutor's office after 4 weeks in accordance with section 170 (2) StPO without investigation measures being initiated. Reasons for the termination cannot be found in the file.

The delivery of the doping substance to the receiver as well as the references to his participation in sporting competitions and his revenue generated thereby should at least have given rise to investigations.

It is also noteworthy that both public prosecutor's offices (in case a and case $b$ ) have closed all proceedings conducted by them for self-doping in accordance with section 170 (2) StPO.

\section{d) Summary penalty orders and sentencing}

Regarding self-doping, the relevant procedural files made available to the experts contained only three summary penalty orders and one acquittal following an appeal against a summary penalty order. In the latter case, a Bundesliga wrestler, who was accused of an offence under section 4 (1) no. 4 in conjunction with (7) no. 1 was acquitted.

On the one hand, it is striking that two of the summary penalty orders were issued against accused who made a confession regarding the subject of the investigations. On the other hand, it is remarkable that two of the sentences imposed in the summary penalty orders are at the lowest edge of the range of sentences: In the case of a baseball player, a fine of 40 daily rates was imposed for an offence under section 4 (1) no. 5 in conjunction with (7) no. 2. In proceedings against a cyclist, a fine of 30 daily rates was imposed for an offence under section 4 (1) no. 3, no. 5, (7) no. 1. In proceedings against a professional boxer who tested positive at a competition abroad, a summary penalty order was issued for a total of 120 daily rates: 80 daily rates for a "violation of section 3 (1) sentence 1 " and 60 daily rates 
for a "violation of section 3 (4)".${ }^{74}$ The accused initially raised an objection (without detailed justification) against the summary penalty order; however, this objection was withdrawn on the day of the main hearing without a plea on the merits.

It was not possible to evaluate the case file of the professional boxer $\mathrm{Fe}$ lix Sturm, who was convicted by the Regional Court of Cologne at the end of April, as the proceedings had not yet been concluded at the time of the evaluation. Of the total prison sentence of three years, six months were imposed for the offences under section 4 (1) nos. 4 and 5, which were committed in coincidence, as well as for the related bodily injury. The written grounds for the verdict were not yet available at the time this report was written.

\section{Evaluation}

a) General evaluation of the criminal law regulation of self-doping

\section{aa) Overview}

The concerns against the introduction of the AntiDopG in general and the criminal liability of self-doping in particular that had been expressed during the legislative procedure have been partially dispelled. It is noticeable that the attitude of the athletes' representatives is more positive than it was during the legislative procedure. It is also apparent that the majority of the public prosecutors interviewed have no concerns about the criminalisation, while the judges interviewed assess the offences more critically; however, the judges have not yet been able to gain any experience with charges of criminal violations of the self-doping ban.

bb) Appropriateness of the criminalisation of self-doping

During the legislative procedure, it has often been criticised that fairness and equal opportunities in sport are values of sporting ethics, ${ }^{75}$ but no le-

74 Why the summary penalty order did not set the sum of 80 daily rates and 60 daily rates (140), but 120 daily rates, was not apparent from the files.

75 DOSB, Opinion on the draft bill for a law to combat doping in sport, p. 4. 
gitimate objects of protection of criminal offences. ${ }^{76}$ These concerns are still hinted at by one athlete's representative:

"I think this is a little difficult. On the one hand the integrity of sport is a valuable commodity for me, but on the other hand it was only introduced by the AntiDopG. I don't know if this is the right place to punish self-doping so much again, because for me as an athlete it is a much higher penalty that you are practically banned from your sport for two years and, in addition, have to pay back all income from the Sports Aid. This is a deep cut in life, which you do not want to take on under any circumstances. (...) That's why I would view it as a little critical to punish self-doping in a national law. It is already punished very severely elsewhere. That is probably not the way to prevent self-doping." (A 1)

Nevertheless, when asked whether it would be useful to prosecute the illicit use of doping methods and substances, she replied as follows:

"I think that it makes sense in general, (...) also from health aspects, especially from the aspect of how our meritocracy develops. There is a high risk if young athletes notice the illicit use of doping methods and substances in their club. In the leisure sector doping also takes place often. (...) That's why I generally think it's not bad that this is being prosecuted. However, you always have to clarify the framework conditions in such a way that it is proportionate." (A 1)

Another athlete representative is also ultimately convinced that the criminalisation would be useful:

"I find it a little bit hard to answer, because personally I was always very ambivalent about whether this is the right way to go. I was worried that this criminalisation takes place, also because I see many situations where young people get into a system, dependencies arise, pressure situations arise. I do not mean to say that they are therefore not guilty when they take doping substances, but there is sometimes a system which strongly favours this and which also puts them in a very difficult situation when they do not take the doping substances, because this partly happens in a group. That's why I was partially skeptical whether these athletes should actually be penalised. They already have to endure a hard punishment, because they actually lose their career. That's what causes my conflict. Basically, though, I believe that hard

76 Opinion of the German Association of Judges on the draft bill for a law to combat doping in sport, no. 7/15. 
measures are necessary. We see that with the means we currently have for prosecution within the Anti-Doping Control System it is very difficult to stop someone from taking doping substances if he or she is willing to take them." (A 2)

The majority of the public prosecutors interviewed also did not express any doubts about the usefulness of the criminalisation and the prosecution of self-doping.

"I consider the offence as very important." (StA 1)

"In terms of the idea of sport, I consider it as quite counterproductive that people who work with doping substances have more training progress and more success. That's why I think that the principle of criminal liability for such matters makes sense, yes." (StA 5)

One public prosecutor, however, expressed concerns:

"I think that everyone must decide on his or her own responsibility whether or not to take doping substances. Recently I had a case, where someone who was subject of an arrest warrant was supposed to be extradited from Georgia (the Georgians don't know the AntiDopG and also have no similar law and everybody is always surprised that this is punishable in Germany). We probably go on a special path. I think that the self-responsibility among athletes should be enough to decide whether to take doping substances or not. Possession of doping substances for personal use - yes, I don't know if that necessarily has to be liable to prosecution." (StA 2)

However, according to the public prosecutor's own statement, he has so far only dealt with one case of self-doping. The same applies to the two judges, who also expressed critical views.

"I never had anything to do with section 3. I have actually not yet developed an informed opinion on section 3 because I have never had to apply it myself. My opinion on it is rather superficial. I consider section 3 to be largely superfluous. (...) From the point of view of criminal prosecution it seems to me completely uninteresting that top athletes apply or have applied doping methods to themselves. I do not know why this should not be allowed. (...) I have my doubts because it is primarily a matter of self-endangerment. The only reason for me to pay attention to this phenomenon is the fact that there are also underage athletes who must be protected by all means." ( $R$ 1) 
Someone else pointed out:

"Why should I stop an athlete from doing that? I mean, although this is a stupid example, I'm not stopping anyone from eating 10 kilograms of beef or pork every day, and somehow harming him or herself thereby. Let's put it this way: I could live with it better if the real sense, the real purpose would be to protect the sport. Doping, some would say, that is also sport. But if I leave aside the fact that this is a way of at least helping to limit the certainly existing doping problem, I am still waiting for the first case. That is a bit strange, because if you look closely that is almost impossible." ( $R$ 2)

cc) Fear of deliberate false accusations

During the legislative procedure, athlete representatives have expressed concerns that athletes could be mistakenly suspected on purpose, for example by competitors, or that a small amount of doping substances could be foisted on athletes. So far, however, athlete representatives are not aware of any such case:

"No, I don't have an illustrative example of that, and I wouldn't dare stating that it unsettled the athletes." (A 2)

"I am not aware of any case." (A 1)

When asked about a possible insecurity of the athletes, a representative of athletes answered:

"No, I do not have an example of that. (...). But that still doesn't mean it's OK. It's just in the perception - I think - not so bad." (A 1)

dd) Concern about the importance of sports jurisdiction

Finally, the empirical study did not reveal any indications that the criminalisation of self-doping and the State prosecution has led to a weakening or devaluation of sports law proceedings. Commenting on this, a representative of an association said:

"It is true that we had indeed always brought this up in the legislative procedure and that it was precisely this weakening that was the greatest concern. Whether this was actually justified, we cannot yet answer clearly. We do not yet see an actual case in Germany that would be such a prominent case inde- 
pendent of popular sport, and is sentenced with penalties and sanctions provided by the AntiDopG. Therefore, we cannot even compare and consequently also not assess if there actually is a discrepancy between the sports law and the criminal law sanction system." (D)

The athletes' representatives even stressed that the fear of consequences under the sports law would be greater than the fear of prosecution. ${ }^{77}$

ee) General evaluation

When asked whether the self-doping ban of the AntiDopG would be successful, the majority of the athletes and public prosecutors interviewed responded with a basically positive tendency. Exemplary:

"I think, as a whole, it is successful. I just think that we really have to get away from saying that the AntiDopG solves the problems." (A 1)

"Yes, I think, as a whole, it is successful. The cases in top-class sport are still within limits, if I understood it correctly, but I do think that it is successful as a whole." (A 2)

"It definitely succeeded to criminally or legally legitimise a really very difficult and very, very controversial area. This is good, this is the right way. Of course, it is also important to look at this law regularly and to improve necessary nuances." $(N)$

"Successful and no change requests." (StA 4)

However, these positive statements are limited by some with a view to the small number of cases:

"Well, I think the AntiDopG is successful. You can't say that it backfired. It has brought about a multitude of positive phenomena. (...) These are all points which support the anti-doping prosecution, although the whole basic trend and situation that you don't get cases in competitive sports did not actually improve because of the AntiDopG. (...) We simply have no cases. The AntiDopG could offer an incentive or provide the possibility to get cases by starting where the information could come from. In my view, it can only come from the scene, and that is why the absence of a sports-specific leniency is

77 See quotations from A 1 on p. 37 and 68. 
a considerable shortcoming; I believe that the AntiDopG has a considerable need for improvement." (StA 3)

"I think it's somehow really difficult for us to evaluate this because we think that there are too few relevant cases to assess whether it works or not. If you I mean, overall that's not desirable, - but if you had ten or twenty actual cases of application to test it on - that sounds a bit weird - but if you could actually completely apply the law, and by that I don't mean just any bodybuilders from any fitness studio, but really these top athletes that we're talking about of course we do not want that, but only then, I think, we could say, "It really proved itself or not." $(D)$

b) Practical proposals for the reform of the criminal law provisions on self-doping

Despite the basically positive assessment of the self-doping ban by the majority of the respondents, the experts made suggestions for the improvement of the criminal punishment of violations against the AntiDopG at various places. In relation to the substantive law, the focus was on the revision of section 4 (7), which was considered too vague, especially with a view to the "significant revenue" (see 2.c. bb. above). However, most frequently the experts pointed out the introduction of a leniency and better protection for whistleblowers.

aa) Introduction of a specific leniency

As already stated, (see 3.a. bb. above), the leniency in section 46b StGB does not apply to self-doping athletes. An independent leniency as in section 31 BtMG does not yet exist in the AntiDopG. Therefore, athletes accused in criminal proceedings have no legal incentive to disclose their own knowledge about structures, backers or other perpetrators.

In their statements, the NADA, the DOSB, the German Lawyer Association (DAV) and athletes associations have called for the introduction of a leniency for athletes. ${ }^{78}$ It is pointed out that the prospect of a reduced sen-

78 Athletes Germany Association (Athleten Deutschland e.V.), opinion at the hearing of the sports committee on August 23, 2019, p. 4; DAV, opinion no. 38/2019, p. 4; DOSB, public hearing of the sports committee on October 23, 2019, p. 2; NADA, public hearing of the Sports Committee of the German Bundestag, Need 
tence or impunity could motivate athletes - who often remain silent out of shame, fear or misconstrued loyalty - to provide further information about their milieu to the investigating authorities. An international comparison has also shown that cooperation with convicted athletes is an important prerequisite for the detection of doping structures.

"The responsible investigators of the Anti-Doping Agencies are facing great problems due to this lack of will to cooperate. They are often neither able to identify all members of the doping network nor to gather sufficient legally effective evidence against suspects. (...) In the past, such mechanisms have proved to be very helpful. Leniencies, for example, helped the American authorities to uncover the widespread abuse of doping substances within the former cycling team US Postal." 79

Also the legal literature demands the introduction of a leniency for all offences provided for in section 4. It is argued with the parallel to the narcotics law, whose special leniency in section 31 BtMG would be comparable in its ratio and would have proven itself in practice. ${ }^{80}$ The criminal leniency should be accompanied by the commitment to lift sports law bans respectively to close the disciplinary proceedings for doping by the competent anti-doping organisation. ${ }^{81}$

In the expert interviews, two positions on the leniency could be distinguished. Some of the experts doubted the need for a special leniency.

"[Whether a leniency would be useful,] I cannot answer, because we very, very seldom deal with competitive sports and I am, to be honest, not aware of any application of a leniency in our investigations." (Zoll 3)

Interviewer: "What role does the leniency applicable to section 2 play?" $R$ 1: $\quad$ "So far, it did not play a role for us."

Interviewer: "Would an extension of the leniency to self-doping make sense?"

$R$ 1:

"Since the leniency has not played a role so far: no."

"As far as I can see, [the leniency] has as yet not played any role for us, I mean in my work. I can therefore not assess that." (StA 5)

for Amendments and Supplements to the Act against doping in sport (AntiDopG), p. 2 f.

79 Opinion Athleten Deutschland e.V., p. 4.

80 Hauptmann/Klarmann, SpuRt 2019, 197.

81 Cherkeh, SpuRt 2019, 167. 
Interviewer: "What role does the leniency applicable to section 2 play?"

StA 7:

"It does not play a role for me."

Interviewer: "Would an extension of the leniency to self-doping make sense?"

StA 7: $\quad$ "No, because the leniency does not play a role for me."

However, the respondents did not criticise the basic idea of a leniency, but did not see any practical reason for a reform in view of their own occupation. Considering that the number of proceedings is currently still low this finding should not be overestimated: Since the public prosecutor's offices - and all the more the courts - have only very rarely been confronted with relevant cases of self-doping, it is obvious that so far there has been hardly any need for a leniency.

Nevertheless, in the interviews, in addition to the representatives of the NADA and athlete federations, also public prosecutors spoke in favour of a special leniency in the AntiDopG.

"A special leniency would certainly be desirable. If the accused could be instructed to that effect, it would, by all means, be a signal for him. He could be motivated to provide further information." (StA 1) $)^{82}$

"In my opinion, the AntiDopG is a good law. You can really work with the AntiDopG, but this is a real loophole. You can really improve on that. (...) I think this is a very central point. If you want to do something about it, if you want to optimise the prosecution of doping, then you have to establish this leniency." (StA 3)

bb) Improved protection of whistleblowers

The opinions of the NADA and the athletes associations call furthermore for an improved protection of "whistleblowers" ${ }^{83}$ This concerns persons

82 However, the expert adds that this is a general assessment which is not based on experience: "As far as the existing possibilities are concerned, we have, however, so far very rarely found that an accused person provides more information simply because he has been given the appropriate instruction, but nevertheless we would consider it as a useful signal for the accused person."

83 Athleten Deutschland e.V., Statement at the hearing of the Sports Committee on August 23, 2019, p. 4; NADA, Public Hearing of the Sports Committee of the German Bundestag, Need for Amendments and Supplements to the Act against doping in sport (AntiDopG), p. 3. 
who wish to reveal information about doping violations without themselves being confronted with a criminal charge. The NADA points out that "all revelations of the biggest current doping scandals [...] are based on the statements of whistleblowers" ${ }^{84}$ The representatives of these bodies emphasised the importance of a better protection of whistleblowers also in the interviews.

Whistleblowers risk serious professional, financial and private consequences by exposing doping violations, as there is still the risk of being considered as a "runner-down" in the sporting environment. ${ }^{85}$

"It is important for us, however, that also people who see something are protected sufficiently. Also, the Russian scandal has shown that there are countries where athletes are exposed to very, very great dangers. Even in Germa$n y$, partially criminal structures are behind it. The people who say something must in any case know that they are protected. Many people don't say anything out of fear. I think this is a big point that needs to be improved." (A 1)

In the interviews, in particular the athletes' representatives pointed out that existing whistleblower systems - such as those of the NADA or the World Anti-Doping Agency (WADA) - are hardly known so far.

"The problem is that it's far too unknown. Most athletes don't even know that it exists. (...) We had a small internal survey among the athletes' representatives, these are people who are already more interested in sports politics and read many more e-mails and obtain information on websites, and of these 35 people only two people - or three people including me - knew about the whistleblower system. You therefore can imagine what it looks like among athletes." (A 1)

In the opinion of the respondents, a good whistleblower system should be completely anonymous and at the same time - e.g. by means of a "mailbox function" - allow follow-up questions to the whistleblower. However, the experts did not make any concrete proposals to the legislator. The establishment of effective whistleblower systems was primarily seen as a task of the federations and anti-doping agencies:

84 NADA, Public Hearing of the Sports Committee of the German Bundestag, Need for Amendments and Supplements to the Act against doping in sport (AntiDopG), p. 2.

85 Hauptmann/Klarmann, SpuRt 2019, 191. 
"Whether the Act against doping in sport is the right place [for provisions regarding the protection of whistleblowers], I don't know. We ourselves launched the initiative to say that whistleblowers in sport must be protected in principle. This is rather a task coming from the sport. (...) I kind of miss a concrete approach on how a legislator can take the lead. Nevertheless, I would of course like to see some initiative, if that is possible." (N)

\section{The prohibition norms of section 2}

Section 2 is one of two central prohibition norms of the AntiDopG. Section 2 (1) contains offences which address the "delivery side" 86 of doping and which prohibit, in particular, the manufacturing, trafficking and placing on the market of doping substances. Section 2 (2) covers the illegal administration or application of doping substances and methods to another person, while section 2 (3) prohibits the purchase and possession of doping substances in significant quantities for the purpose of doping humans and their transport to or through the territory governed by the AntiDopG. Section 4 (1) nos. 1 to 3 makes violations of these prohibition norms liable to prosecution.

According to all interviewed persons who work at customs, the public prosecutor's offices and the courts, these provisions are at the centre of their practical work with the AntiDopG. The following statement of a public prosecutor is representative:

"The violations of section 2, i.e. the possession of doping substances in significant quantities, are the focus of our work, of our preliminary investigations. Without this provision we would have little contact with the AntiDopGt itself (...).” (StA 6)

This statement is confirmed by another public prosecutor, who at the same time shows which group of persons makes up the majority of the accused:

"90\% of the cases are of course that at Frankfurt airport customs finds many parcels containing testosterone ampoules or similar, which were purchased by a recreational atblete via an Internet portal (...) and which are usually shipped to Frankfurt from Thailand, China or the USA and are then intercepted by customs. Of these $90 \%$, I would say: $80 \%$ are recreational athletes who go to the gym, who build up their muscles." (StA 2)

86 Accurate Weber BtMG/ibid., 5th ed. 2017, AntiDopG $\$ 2$ Rn. 2. 


\section{Phenomenon}

a) Findings on perpetrators and criminal acts

The statements made by the interviewed investigators of customs, the public prosecutor's offices and judges on the accused were uniform. According to them, the proceedings concern almost without exception "the fitness scene in general, so let's say mass sports, typical gym visitors, i.e. weight training and bodybuilding." (Customs (Zoll) 3). The "area of muscle building, bodybuilding" would "really (be) the biggest part of our proceedings." (StA 1)

This information can be split up in two respects. On the one hand, the preliminary investigations are not only directed against strength athletes who "really do that competitively, but also (against) people who simply want to get their bodies in shape. That's the bulk of cases we have." (StA 5)

"The predominant portion of the proceedings that we deal within the specialised public prosecutor's office are proceedings involving bodybuilders or weight training. Some of the bodybuilders practice the sport competitively, but the majority of the bodybuilders are simply people who go to the gym and do the sport there. That's the largest part in terms of numbers." (StA 4)

"The main recipient is clearly the bodybuilder sport. Starting with people who do it actively, i.e. who compete, but also a large proportion of recreational athletes in the gyms, who expect increase in strength to match the current lifestyle. And that's over $90 \%$ of our group of recipients." (Zoll 1)

On the other hand, the interviews show that self-users make up the majority of the accused, i.e. persons who purchase, possess or carry to the territory governed by this act doping substances for their own use. Whereas those who exclusively or primarily deliver doping substances to third parties, manufacture them or traffic in them are clearly underrepresented. Thus, the prosecutors focus "clearly on self-users" (StA 7); the accused are predominantly "only consumers" (R 1).

It happens less often that preliminary investigations are directed against manufacturers or dealers. One interviewee told about a special case of manufacturing of doping substances and commercial trafficking:

"A concrete case, which is actually somewhat larger, is a married couple who ordered substances from China over a period that is not even that long. Raw materials from China. And then - without much previous knowledge of chemical processes - created mixtures, mainly testosterone products, and then sold them on eBay to a significant extent." (StA 5) 
More frequent, however, are cases in which an accused has acquired or possesses doping substances for his or her own use and at the same time traffics in them (to a small extent). A judge commented on that:

"It was either the bodybuilding studio operator, who also took the stuff himself and passed it on to his customers, or the bodybuilder himself. I think there were one or two who ordered it in bulk and then resold it, so that they ended up committing a serious criminal offence. But basically, it is the typical self-harmers." ( $R$ 2)

"Trafficking, that happens from time to time, like the narcotics addicts when they buy something and give away parts of it to get the money for the next batch." (R 2)

Nevertheless, structural parallels to other forms of organised crime are emphasised by customs investigators and also individual public prosecutors, in particular a "collaborative, very conspiratorial approach". (Zoll 3). There are "also frequently points of contact with the rocker scene." (Zoll 3) Especially the high profit margins are mentioned as a reason why the organised trafficking in doping substances is interesting for offenders:

"Yes, clearly. The profit margin in trafficking in doping substances is higher than in trafficking in narcotics. Until a few years ago, the threat of punishment was also much lower, so it could be assumed that trafficking in doping substances was safer. One was able to generate more profit than with drug trafficking, and the punishment was also not at all comparable to the punishment of trafficking in narcotics." (Zoll 1)

If there are corresponding indications of organised offender groups, the investigators try to uncover these networks:

"Of course, we're trying to get the backers. We're trying to investigate in particular at this point. From a certain amount of import volume or also from the products, one can estimate whether the recipient is a consumer or someone who processes it. Our endeavour is to get the backers, especially those who make a profit out of it, i.e. who process the doping substances and then ultimately traffic in them." (Zoll 1)

"We have also already stopped large freight shipments at Frankfurt Airport, which should then be, so to speak, obtained by an operator network. These do exist, but in a small number of cases. It happens, one could say, every year and a half that we make investigations of structures into such large cases." (StA 7) 
b) Findings on doping substances

The questionings on the doping substances subject of the proceedings revealed three essential findings. On the one hand, classical medicinal products for muscle building dominate:

"Ultimately, we start with the classic, the testosterone, nandrolone, but also the stuff you need to cushion the side effects from tamoxifen to clomiphene. Everything you can think of. Our main group of perpetrators operates in recreational sports, i.e. weight training, bodybuilding - and everything that causes muscle growth is ultimately imported, partly as a finished product, partly as a raw material, as an active substance, which is then further processed into these corresponding doping substances." (Zoll 1)

On the other hand, according to the investigators, trends can also be observed as a result of which new doping substances quickly become very popular:

"There are lots of new fancy substances. (...) For quite some time now lots of the new SARMs [selective androgen receptor modulators], for example." (Zoll 3)

Thirdly, investigations increasingly concern lifestyle drugs, which can in principle be used for the purpose of doping in sport, but often serve other purposes:

"Recently this DHEA. And epiandrosterone (...) also is very common. Whereby it is then used less for muscle building, but to regain control of the normal aging and sexual desire at a certain age." (StA 2)

According to a customs investigator, proceedings involving such lifestyle drugs cause considerable expense. The concrete determination whether they are actually intended to serve doping purposes would be labour-intensive and sometimes hardly determinable.

"We are very much frequented by it. (...) However, although it is listed and we then (...) have to conduct the preliminary investigation, (...) we have no knowledge that this medicinal product was even intended to be used for such purposes. (...) That ties up a lot of personnel." (Zoll 2) ${ }^{87}$

87 More on the difficulties in determining the intended use below 2.a.ee. 


\section{B. Results of the evaluation}

c) Summary

At the centre of the prosecution of offences under the AntiDopG are violations of section 2. Within this group of violations, the investigations are mostly directed against self-users of doping substances who do bodybuilding and fitness as a popular sport outside of competitions. Accordingly, the vast majority of the doping substances subject of the proceedings are medicinal products used for muscle building. In addition, there are an increasing number of new "fancy" medicinal products or lifestyle products which are often not used for doping purposes.

2. Findings on substantive aspects of section 2

a) The offences and their prerequisites

aa) Legislative context

Pursuant to section 2 (1), it is prohibited to

- manufacture,

- traffic in,

- sell, dispense or otherwise place on the market without trafficking, or

- prescribe.

A doping substance which is or contains a substance listed in Annex I of the International Convention against Doping in Sport of 19 October 2005 in the version promulgated by the Federal Ministry of the Interior in part II of the Federal Law Gazette for the purpose of doping human beings in sport.

Section 2 (2) prohibits to administer to another person such a doping substance for the purpose of doping in sport. Finally, section 2 (3) prohibits to purchase, possess or carry to or through the territory governed by the AntiDopG significant quantities of such a doping substance which is or contains a substance listed in the annex to this act, for the purpose of doping in sport.

Section 4 (1) nos. 1 to 3 makes actions contrary to these prohibitions punishable, whereas section 4 (6) also makes negligent conduct punishable. 
According to the legislator, these provisions "serve primarily the protection of health" ${ }^{88}$ This would be because the use of doping substances and doping methods for the purpose of doping in sport would not be based on a medical indication and therefore would lead to an intervention in the body which is not medically indicated and which entails considerable risks for the health of the athletes concerned. Numerous deaths in the past and serious long-term consequences of systematic doping would prove the harmfulness of doping. Underage athletes would also obtain and use doping substances despite the special health risks that the use of these substances holds particularly for young people. ${ }^{89}$ Not only the athletes would be affected but also the general public, which bears the costs of treatment. In addition, the explanatory memorandum to the Act refers to the illegal trafficking in doping substances, which would have reached alarming dimensions and would use organised distribution channels and dealer structures comparable to those in the organised drug trafficking.

Section 2 adopts the prohibition norms previously contained in the AMG and places them - in a modified form - in the context of doping in sport. The spin-off of the prohibition norms from the AMG and their integration into the AntiDopG is assessed positively:

"[Our work] has become easier in the sense that the application of law has become easier and we no longer have to rummage through the Medicines Law." (Zoll 3)

The greater clarity of the norm is also emphasised:

"The (prohibition norms) are very brief and very clearly understandable (...).” (Zoll 1)

One advantage of the new provision is seen in the fact that the applicability of the prohibition norms is now no longer linked to the properties of a medicinal product, but to the broader term of "doping substance". An investigator commented on the previous legal situation under the AMG:

88 BT-Drs. 18/4898, 23; Erbs/Kohlhaas/Wußler, 228 EL January 2020, AntiDopG $₫ 2$ Rn. 1.

89 BT-Drs. 18/4898, p. 53; Eising, Die Strafbarkeit des Eigendopings, 2018, p. 86; Heger, medstra 2017, 205, 212; cf. in this respect also the parallel provision of section 29a (1) no. 1 BtMG on the illicit distribution of narcotics to minors: Körner/ Patzak/Volkmer/Patzak BtMG, 9th ed. 2019, $\$ 29$ a Rn. 5. 
"In order to achieve criminal liability, we had to 'deal' with the fact that the doping substances are often counterfeit medicinal products. In order to achieve a conviction, we had to use auxiliary bridges at that time." (Zoll 1)

The act does not define the term of "doping substance". However, the explanatory memorandum to the Act states that both medicinal products and other active substances should be covered..$^{90}$ The decisive factor for the applicability of section 2 (2) and (3) is that doping substances contain substances listed in Annex I of the International Convention against Doping in Sport of 19 October 2005, in the version promulgated by the Federal Ministry of the Interior in part II of the Federal Law Gazette. Therefore, in contrast to section 6 a (2) sentence 1 of the old version of the AMG, not all amendments to Annex I of the Convention are included in the prohibition. Rather, such amendments at the international level become domestically effective for the AntiDopG only through a decision of the national regulator. ${ }^{91}$

In a decision of 14 February 2019, the Federal Court of Justice (BGH $)^{92}$ considered this to be a sufficient "degree of national design sovereignty" and for this reason rejected constitutional objections to the reference to Annex I of the International Convention against Doping. ${ }^{93}$ Practitioners assess this approach as positive because "with the help of the list of substances and the list of banned substances" it would be easy to determine the range of criminal liability (Zoll 1).

Critics complain, however, that - in contrast to the BtMG, for example no statutory instrument would be required to determine the incriminated medicinal products and active substances, but a mere announcement of the adoption of the application of amendments to Annex $1 \mathrm{~d}$ of the Doping Convention: A statutory instrument would have - also with regard to the requirement of the principle of legality - a different status than an announcement and, in addition, different procedural requirements. ${ }^{94}$ Therefore, it is demanded that a new power to issue statutory instruments for

90 BT-Drs. $18 / 4898$ p. 23; critical Weber BtMG/ibid., AntiDopG $₫ 2$ Rn. 6.

91 BT-Drs. 18/4898, p. 24.

92 BGH of 14 February 2019, 4 StR 283/18, StV 2020, 315, 317. See also Finken, PharmR 2016, 445, 446: "The provision therefore does not meet with any constitutional concerns."

93 BGH of 14 February 2019, 4 StR 283/19, StV 2020, 315, 317.

94 Weber BtMG/ibid., AntiDopG, 5th ed.17, $\$ 2$ Rn. $14 \mathrm{f}$. Furthermore Graf/Jäger/ Wittig/Eschelbach, Wirtschafts- und Steuerstrafrecht, 2nd ed. 2017, $\$ 4$ AntiDopG Rn. 1, 11. 
the specification of incriminated substances is created in the AntiDopG in order to determine the objects of the AntiDopG from now on more precisely by way of a statutory instrument - instead of an announcement:

"Also in the fight against doping in sport, it would at least be a gain in the rule of law if such a power to issue statutory instruments would be created or, as long as this has not been done, instead of an announcement the power to issue statutory instruments under section 6 (2) would be used." 95

The legislator, on the other hand, points out that precisely in the fight against doping special flexibility would be needed in order to be able to react quickly and appropriately to new developments - even "during the year" and within a few months. In the past, it would have shown that "there is a high risk of lagging behind the rapid development of new doping substances and doping methods, which impairs the effective fight against doping. ${ }^{96}$

bb) Manufacturing, trafficking, placing on the market, prescribing (section 2(1))

According to section 6a AMG (old version), the placing on the market, prescribing or administering of medicinal products to others for the purpose of doping human beings in sport was prohibited. These prohibitions have been incorporated into section 2 (1) in an altered form and new modi operandi have been added. ${ }^{97}$ According to the legislator, the provisions of the AMG had proved to be too narrow; the manufacturing, trafficking, selling and dispensing of doping substances should also be punishable bearing in mind the health risks caused. ${ }^{98}$

The ban on manufacturing is intended to combat the development of illegal markets. ${ }^{99}$ Due to its great practical importance, the legislator decided to include the selfish, turnover-oriented act of trafficking. ${ }^{100}$ By incorporating the offence of selling, those cases in which the perpetrator dis-

95 Weber BtMG/ibid., 5th ed. 2017, AntiDopG $\ 2$ Rn. 15.

96 BT-Drs. 18/4898, p. 25.

97 Weber BtMG/ibid., 5th ed.2017, AntiDopG, $\mathbb{2}$ Rn. 6.

98 BT-Drs. 18/4898, p. 23; Lehner/Nolte/Putzke/Striegel, AntiDopG, $\mathbb{2}$ Rn. 12.

99 BT-Drs. 18/4898, p. 23; Graf/Jäger/Wittig/Eschelbach, Wirtschafts- und Steuerstrafrecht, 2nd ed. 2017, AntiDopG, $₫ 4$ Rn. 5.

100 The term is derived from the BtMG, BT-Drs. 18/4898, p. 24; Weber BtMG/ibid., 5th ed. 2017, AntiDopG, $\mathbb{2}$ Rn. 35. 


\section{B. Results of the evaluation}

penses doping substances against payment but unselfishly will be covered. ${ }^{101}$ In order to address regulatory loopholes for acts of disposal that cannot be specifically proven, the catch-all element of other placing on the market has been included in the prohibitions. ${ }^{102}$

The interviewees appreciated the new version for two reasons. First of all, it would remove previously existing ambiguities in interpretation or close legal loopholes. The inclusion of trafficking is seen as particularly positive, because some cases are not yet covered by the act of placing on the market:

"Including the act of trafficking was a good decision. The act of placing on the market has always required some kind of stockpiling so that any incidents beforehand, such as a mere order or an intercepted package, have always lacked a legal basis." (StA 3)

The new legal situation would bring about a simplification ${ }^{103}$ as it would also cover "verbal trading up until a serious binding agreement has been reached" (StA 3) and thus all cases "from packing a parcel that is not even sent away in the end through to an intercepted parcel" (StA 3).

It is also appreciated that the wording of the law is based on established formulations of the BtMG:

"The new legal situation is to be welcomed because of its similarities with the BtMG."

On the one hand, this would be important for defence attorneys and other legal practitioners when they have "to deal with violations of the AntiDopG for the first time" and are "more familiar" with the BtMG. On the other hand, referring to well-known terms "that have already been shaped by the case-law on the BtMG" would be advantageous. (StA 1)

The benefits to the prosecution authorities are summarized as follows:

"Most importantly, section 2 is clearly defined. In the end, it is very similar to the BtMG." (StA 5)

101 BT-Drs. 18/4898, p. 24; MüKo-StGB/Freund, 3rd ed. 2018, AntiDopG, $\$ \$$ 1-4 Rn. 50.

102 BT-Drs. 18/4898, p. 24; Weber BtMG/ibid., 5th ed. 2017, AntiDopG, $\mathbb{2}$ Rn. 55.

103 "In my opinion, this was a huge relief." (StA 3) 
cc) Administration or application of doping substances and methods (section 2(2))

The prohibition of administering doping substances (no. 1) corresponds with the previously applicable prohibition under section 6a (1) AMG. The new provision has been extended to include the prohibition of administering doping methods to another person (no. 2). The doping methods - as well as the doping substances -are listed in Annex I of the International Convention against Doping in Sport in the version promulgated by the Federal Ministry of the Interior.

In order to address legal loopholes, the legislator explicitly included doping methods. ${ }^{104}$ This is appreciated by a public prosecutor who is referring to a case example as follows:

"It was a good decision to include doping methods and thus close any legal loopholes. (...) In a previous trial, we discovered that unlawful methods were applied and thanks to the new provision, we were able to issue an order of summary punishment. Otherwise, we would have been compelled to close the proceeding." (StA 1)

Another public prosecutor agrees with this and refers to legal uncertainties in the previous legal situation under the AMG by citing a specific preliminary investigation:

"The inclusion of doping methods was really important. Under the AMG the precise distinction between methods and medicinal products was always a matter of dispute, especially in terms of blood doping like in 'Operation Aderlass'." (StA 3)

Only one judge, who has not yet gained any experience in this matter, expressed criticism:

"I have my doubts as to whether this is really necessary because we never had to negotiate the use of certain doping methods." $(R 1)$

104 BT-Drs. 18/4898, p. 25; Körner/Patzak/Volkmer/Volkmer, BtMG, 9th ed. 2019, AntiDopG, Vor $\mathbb{1} 1$ Rn. 21; Weber BtMG/ibid., 5th ed. 2017, AntiDopG, $\mathbb{1} 1$ Rn. 2. 
dd) Acquisition, possession and transfer pursuant to section 2 (3)

(1) General meaning

The prohibition of possessing and purchasing significant quantities of certain health-endangering doping substances for the purpose of doping in sport contained in the previously applicable section 6a (2a) sentence 1 AMG has been incorporated into section 2 (3). Additionally, the prohibition of carrying to or through the territory governed by this act, thus to or through the territory of the Federal Republic of Germany, has been included. Thereby, the prohibition of carrying medicinal products to the purview of this Act (section 73 AMG) is supplemented by the act of carrying doping substances through the purview of this Act.

(2) Acquisition and possession of significant quantities

In practice, the acts of acquisition and possession of doping substances seem to be of particular importance. This is indicated by the statements quoted above under $1 \mathrm{a}$ ), according to which the accused are "definitely selfusers" (StA 7) in most cases. When asked about a typical example of his investigations, a customs investigator states the following:

"The focus is on section 4 (1) no. 3 in conjunction with section 2 (3). We operate in accordance with it." (Zoll 2)

The legislator imposed a ban on the acquisition and possession of significant quantities of doping substances, since experience has shown that such quantities usually result in trafficking with doping substances. ${ }^{105}$ The prohibitions contain the legally protected right to public health as the population should be protected from the distribution of doping substances for the purpose of doping. To this end, acts that typically only serve to prepare for transfer should already be prohibited ${ }^{106}$. Thereby, any evidentiary problems that arise in cases where trafficking, selling or dispensing cannot be specifically proven will be solved at the same time.

105 BT-Drs. 18/4898, p. 25; MüKo-StGB/Freund, 3rd ed. 2018, AntiDopG, $\$ \$$ 1-4 Rn. 58; Körner/Patzak/Volkmer/Volkmer, BtMG, 9th ed. 2019, AntiDopG, Vor \$1 Rn. 21.

106 BT-Drs. 18/4898, p. 25 f.; BGH StV 2018, 302. 
The element "significant quantities" is of fundamental importance to this legitimation model. ${ }^{107}$ If the threshold for significant quantities is exceeded, the law will - according to its ratio legis - irrefutably presume the criminal intent to transfer; below this threshold personal use cannot be ruled out and thus, a criminal intent to transfer cannot be presumed. ${ }^{108}$ In general, this approach is approved by the prevailing view in literature. ${ }^{109}$ Occasionally, however, constitutional concerns regarding the principle of legal certainty (Article 103 (2) of the Basic Law) are raised because section 6 (1) authorises the Federal Ministry of Health to determine the significant quantity by statutory instruments without it being clear what their determinations should be based on. ${ }^{110}$ The $\mathrm{BGH}$, however, considers the provision to be constitutional. Since the legislator himself precisely determined the criminal act and the sentencing range, the determination of a significant quantity would merely be a specification of the elements that justify the punishment. ${ }^{111}$ During the interviews, no concerns about the jurisdiction of the regulator were raised either.

However, the evaluation has shown that the application of law clearly distances itself from the above-mentioned basis for legitimacy, namely the prohibition of purchase and possession in order to prevent trafficking in doping substances and not as an end in itself. This is due to two reasons. Firstly, according to the BGH, section 2 (3) shall also cover possessions for the purpose of self-doping: Even if the accused was in possession of doping substances for the mere purpose of self-doping and was at most willing to pass it on unprofitably, he should still be held criminally liable in accordance with section 4 (1) no. $3 .{ }^{112}$ This means that the provision can also be applied to self-doping competitive athletes who do not meet the personal requirements for the application of section 4 (7) and who only possess doping substances for their own purposes. Secondly, many respondents pointed out that the threshold for a significant quantity is extremely low. There-

107 On this and on the following Graf/Jäger/Wittig/Eschelbach, Wirtschafts- und Steuerstrafrecht, 2nd ed. 2017, AntiDopG, $\$ 4$ Rn. 17.

108 Weber BtMG/ibid., AntiDopG, $\mathbb{2}$ Rn. 21.

109 Graf/Jäger/Wittig/Eschelbach, Wirtschafts- und Steuerstrafrecht, AntiDopG, $\mathbb{} 4$ Rn. 17.

110 Graf/Jäger/Wittig/Eschelbach, Wirtschafts- und Steuerstrafrecht, AntiDopG, $\mathbb{} 4$ Rn. 1; Körner/Patzak/Volkmer/Volkmer, BtMG, 9th ed.2019, AntiDopG, $\mathbb{} 4$ Rn. $50 \mathrm{f}$.

111 BGHStV 2020, 315, 317.

112 BGHStV 2018, 302. 
fore, a public prosecutor expressed fundamental doubts about the legitimacy of the norm:

"The legislator was probably struggling due to the fact that doping is generally a self-destructive matter. (...) But anyhow he presumed that the possession of a significant quantity always involves the risk that these doping substances may be placed on the market. (...) This just means that the legislator was uncertain whether the mere possession of doping substances should be punishable. So he decided that it should at least be punishable if there was a risk of trafficking or dispensing. That seems to be ambivalent, I guess. Also, considering my sense of justice, I'm not sure whether this should really be criminalised bearing in mind that a small quantity is really not that much (...)." (StA 5)

Other interviewees confirmed that the threshold for a significant quantity would quickly be exceeded and that no great practical importance would be attached to this restriction of criminal liability:

"In fact, the significant quantities are reached very quickly." (StA 7)

"In martial arts, the significant quantities are quite often exceeded. (...) A $10 \mathrm{ml}$ ampoule, i.e. a perforable ampoule (...), will often be enough to give rise to criminal liability." (StA 3)

"It's relatively rare that someone is in possession of such a small quantity that he will go unpunished. (...) Usually, people have a larger quantity because otherwise it doesn't really make sense to use it for doping." (StA 4)

"Well, the significant quantity isn't much of a problem. In the AntiDopG, the threshold is set so low that almost any possession constitutes a criminal offence. One must rather say that sometimes it is too low so that even the smallest amounts are punishable (...)." (Zoll 1)

Only one public prosecutor stated that it can be difficult sometimes to calculate whether there is a significant quantity of a doping substance:

"Recalculating all of this is quite a lot of work for the prosecution." (StA 1)

If, for example, only one ampoule is found, there will certainly be sufficient initial suspicion under section 2. But whether an infringement of section 2 has actually occurred would depend on how much active substance is still left in the ampoule. That would have to be precisely calculated:

"Just because you have found an ampoule you cannot simply assume a violation of the AntiDopG. Instead, you have to calculate exactly how much is 
still left in the ampoule. Often, you have to obtain an expert opinion for this." (StA 1)

Ultimately, however, this opinion also confirms that the thresholds for significant quantities are set so low that the detection of an ampoule (that is not even completely filled) already provides grounds for an investigation. This means that, above all, self-users who only administer the doping substances themselves and who - contrary to what is assumed in the explanatory memorandum to the Act - do not intend to trade with, sell or dispense doping substances will be prosecuted. The proceedings are often directed against bodybuilders who do not participate in competitions and thus do not use doping substances in order to gain an advantage in sports competition, but for other (e.g.: aesthetic) reasons.

\section{(3) Carrying to or through the territory governed by this act}

The new ban on carrying doping substances to the territory governed by this act already allows for the seizure of doping substances on entry at the border of the Federal Republic of Germany ${ }^{113}$ and enables the prosecution to punish it as a criminal offence. Carrying doping substances through the territory governed by this act is also punishable.

Two public prosecutors expressed a positive opinion on these modi operandi. The "offences of carrying to or through the territory governed by this act" have been "advantageous for us" (StA 1) or rather "absolutely crucial" (StA 7). The reason for this is that the previously required "import" was only given when the goods were released into free circulation after customs clearance. However, if this was not given due to the fact that the goods were not delivered to Germany, but have only been handled there and are being further transported, there was no punishable import. Thereby, legal loopholes occurred:

"Actually, 'carrying to or through the territory governed by this act' is a customs term. It is necessary, though, because the previous AMG has only referred to 'import'. And 'import' requires the entry of medicinal products into free circulation, which will usually not be the case if it has already been detected by the customs authorities beforehand. Since there was no criminal liability for attempted import under the previously applicable $A M G$, the ac-

113 On this and on the following BT-Drs. 18/4898, p. 25; Lehner/Nolte/Putzke/Striegel, AntiDopG, $\mathbb{} 2$ Rn. $83 \mathrm{f}$. 
cused often remained unpunished. By incorporating the act of carrying doping substances to the territory governed by this act, this legal loophole has been closed." (StA 7)

The interviewed customs officers referred to its practical significance as follows:

"For example, if goods are sent from China to Poland and customs controls are carried out at Frankfurt airport, the goods are carried to Germany. And this can already incur criminal liability. So, it had a huge impact on our work." (Zoll 1)

"It's a clarification and it has certainly helped a lot." (Zoll 3)

According to the two of the interviewed customs officers, the provision is considered to be of significant practical relevance:

"Since then, the number of preliminary investigations into cases of transit has increased." (Zoll 3)

"We have a great number of criminal proceedings for transit. Usually, large quantities of doping substances that were seized when being carried to Germany are involved. Some of the criminal proceedings are then continued in other European countries (...).” (Zoll 1)

Another customs investigator, however, pointed out that it is particularly difficult here to distinguish permissible forms of carrying to or through the territory governed by this act from criminal acts because the required element "for the purpose of doping in sport" would not be inherent in the doping substances themselves, but could only be determined through further investigations. The purpose of doping would "sometimes be a bit difficult to identify". Occasionally, it would also concern "larger consignments of medicinal products" (Zoll 2). In such cases transnational investigations may become necessary:

"If doping substances are carried through Germany in order to reach the receiver in a third country, the investigator will have to initiate criminal investigations to find out about the intended purpose." (Zoll 2)

While the ban on carrying to or through the territory governed by this act is considered to be of significant practical importance for the customs authorities, it has played virtually no role in criminal justice so far. One interviewed judge had no experience with charges of illegal transit, which indicates a high rate of termination of proceedings: 
"Well, we've never had any cases of transit. But we've had many cases of manufacturing and trafficking." ( $R$ 1)

Currently, the ban on carrying to or through the territory governed by this act seems to primarily serve the purpose of checking suspicious deliveries as well as seizing doping substances.

ee) For the purpose of doping human beings in sport

The standards of section 2 and the related criminal offences listed in section 4 (1) link the prohibitions or rather the criminal liability for handling or application of doping substances or methods to the "purpose of doping human beings in sport". ${ }^{114}$ The term "sport" shall include all sporting activities; competitive sport as well as popular sport. ${ }^{115}$ Thereby, the improving performance in bodybuilding by means of unnatural increases in weight and strength is also included. ${ }^{116}$

While the classification of activities under the broad term of sport does not cause any practical difficulties, ${ }^{117}$ a proof of the purpose of doping can be difficult. This concerns cases of carrying medicinal products through the territory governed by this act (see above dd. (3)) as well as those lifestyle drugs already mentioned above (under 1.a.). In this regard, the survey indicates that the investigation's main focus lies on the question whether the medicinal product should serve as a doping substance "for the purpose of doping human beings in sport" or whether it is used for other permissible - reasons.

"At the moment, we see a medicinal product called DHEA (dehydroepiandrosterone) a lot. (...) It's one of those lifestyle drugs that has already been approved in the US and is sometimes prescribed by German doctors, too. (...) It can be used to help with cancer, stress, anti-aging or menopausal disorders. (...) Due to this medicinal product, we've got so much work to do. But it might not even be that effective in this respect because the people usually have no clue that it is

114 Section 2 (2) refers to the purpose of doping in sport "to another person".

115 Körner/Patzak/Volkmer/Volkmer, BtMG, 9th ed. 2019, AntiDopG, $\mathbb{4}$ Rn. 24; Erbs/Kohlhaas/Wußler, 228 EL January 2020, AntiDopG, $\$ 2$ Rn. 6.

116 BGH NStZ 2010, 170.

117 The question whether so-called e-sports can also be seen as sport is currently disputed in legal literature, see Kubiciel, ZRP 2019, 200, 203. 
a medicinal product. Nevertheless, preliminary investigations are initiated." (Zoll 2)

If someone is found to be carrying such medicinal products on entry, the investigations seem to be carried out by inspection and queries. An investigator vividly described that his colleagues are then wondering:

"Could the person in front of us be an athlete? (...) What is he or she saying? If the person in front of us is a seventy-year-old pensioner on his travels carrying DHEA with him, I would ask him: what for? If he says that he just came from the US, suffers from a medical condition and that this is why he is taking the pills, I wouldn't have an initial suspicion. I would probably tell him to keep it because you are allowed to import a three months' supply of medication for your own use."118

Such an initial assessment cannot always be made through a mere inspection. Sometimes, the investigations become more complex and investigative resources must be pooled, especially due to the fact that quite a few of the accused happen to exchange defence strategies on the internet in order to convince investigators that the medicinal product is not used for the purpose of doping in sport:

"When talking with the accused, I can't help but wonder what nonsense he or she has been reading on the internet in order to convince me of their innocence." (Zoll 2)

ff) Criminal liability for negligence in accordance with section 4 (6)

Based on the previous section 95 (4) of the AMG, section 4 (6) covers the negligent conduct of the offences under subsection 1 nos. 1 to 3 . The criminalisation is justified by the legislator with regard to the "health endangering" nature of these offences by comparing section 4 (1) nos. 1 to 3 to such different crimes like negligent arson (section $306 \mathrm{~d} \mathrm{StGB}$ ), driving under influence of drinks or drugs (Section $316 \mathrm{StGB}$ ) and negligent bodily harm (Section 229 StGB). Therefore, a criminal liability for negligence seemed to be "appropriate". Especially in those cases where the offender did not consider the respective substance to be a doping substance within the

118 The interviewee was not mentioned here in order to avoid any conclusions about his or her identity. 
meaning of this Act, the criminal liability for negligence should become relevant. ${ }^{119}$

Quantitatively speaking, however, the significance of section 4 (6) appears to be extremely low.

"We once had a conviction for negligent conduct. (...) One case, that's about it." (R 1)

"I haven't really dealt with offences by negligence that much so far." (StA 5 and also StA 1)

The reason given for this is that negligent purchase or possession of doping substances for the purpose of doping human beings is barely conceivable.

"That's due to the fact that it's hard to imagine how someone would negligently obtain such substances. I mean, when you order these products, you will most certainly do so for a particular reason." (StA 5)

In addition, the interviewees reported that in those cases where a negligent conduct is even worth considering, the criminal proceedings are usually terminated though because the offender's guilt is considered to be minor. Therefore, section 4 (6) would play "no major role in practice, since sections 153 et seq. StPO are usually applied in such cases". (StA 3)

Nevertheless, the criminal liability for negligence is welcomed for various reasons. One prosecutor gives a symbolic-communicative reason:

"In practice, offences by negligence have not played a major role so far, I guess. (...) Nevertheless, I consider them to be an important sign. It is thereby made clear that negligent conduct is also punishable." (StA 1)

Two other public prosecutors consider offences by negligence to be much needed catch-all elements for cases where the criminal intent cannot be proven:

"Section 4 (6) certainly is an important catch-all provision." (StA 2)

"They are important because there always has to be a criminal liability for negligence in case the perpetrator does not act with oblique intent (...)." (StA 3)

Another prosecutor argues that a catch-all element would not be necessary in practice, since "at least oblique intent can usually be assumed". (StA 2)

119 BT-Drs. 18/4898, p. 25; Graf/Jäger/Wittig/Eschelbach, Wirtschafts- und Steuerstrafrecht, AntiDopG, $\mathbb{} 4$ Rn. 34. 
b) Penalty enhancing offences

According to section 4 (1), infringements of the prohibitions under section 2 are punishable with up to three years' imprisonment or a fine. Based on this, section 4 (4) creates penalty enhancing offences where the penalties range from one to ten years of imprisonment. They are serious criminal offences within the meaning of section 12 (1) StGB. The aggravating factors shall cover particularly reprehensible and socially harmful conduct. ${ }^{120}$ In terms of content, they correspond to the examples of especially serious cases contained in the previously applicable section 95 (3) no. 1 and section 2 AMG, but are complemented by selling doping substances to a person under the age of 18, prescribing doping substances or applying doping methods to such a person. According to the explanatory memorandum to the Act, the reason given for this modification into a penalty enhancing offence is that "there are nearly no cases where an aggravation seems inappropriate even though all the elements are present. ${ }^{121}$

In practice, the aggravation is welcomed, not only because of the practical consequences, but also because of their symbolic meaning:

"As the AntiDopG has also included serious criminal offences, doping is no longer treated as a trivial offence. So, it has helped to bring the AntiDopG closer together with the BtMG. And this really does affect our practice (...)." (StA 5)

"I particularly appreciate that acting commercially referred to in section 4 (4) has been classified as a serious criminal offence. It just shows that the legislator intends to punish doping offences more harshly. And this seems reasonable to me." (StA 1)

Also with regard to the limitation period, the provision is considered necessary:

"Due to the fact, that section 4 (4) is now a serious criminal offence, the limitation periods have been extended to ten years. Under the previously applicable section 95 AMG, however, there was a limitation period of only five years for especially serious cases." (StA 3)

120 BT-Drs. 18/4898, p. 31; Weber BtMG/ibid., 5th ed. 2017, AntiDopG, $\mathbb{} 4$ Rn. 282; Graf/Jäger/Wittig/Eschelbach, Wirtschafts- und Steuerstrafrecht, 2nd ed. 2017, AntiDopG, $\mathbb{} 4$ Rn. 35.

121 BT-Drs. 18/4898, p. 30. 
Furthermore, it is referred to the possibility of telecommunications surveillance as a much needed special investigation measure:

"Anyone who acts commercially or as a member of a gang has already been held criminally liable under the AMG. But now, there's the possibility of telecommunications surveillance for cases pursuant to section 4 (4) no. 2 lit. $b$ as referred to in section $100 a$. This is a huge advantage from our perspective. Other than that, nothing has really changed." (Zoll 1)

Furthermore, the modification of the previous examples of especially serious cases into serious criminal offences has led to the consequence that the criminal offences under section $4(4)$ are referred to as unlawful acts within the meaning of section 261 (1) sentence 2 StGB and can thereby act as prior offences of money laundering. However, this aspect seems to have had little impact so far; the interviewed practitioners did not report any cases. This could also be due to the fact that the majority of criminal proceedings are conducted against self-users, where following offences such as money laundering are practically ruled out. One investigator suggested yet another reason for this finding:

"It is a big problem. In terms of money laundering the estimation of profits still causes problems because we need to prove at which price the products were purchased and what price they have been sold for." (Zoll 1)

In addition, there are investigative difficulties, for example when social media channels are used for communication:

"It's very difficult to estimate the perpetrator's profit when there are no records due to the fact that he or she is only communicating on WhatsApp." (Zoll 1)

One of the public prosecutors thought about the inclusion of trafficking during which the offender carries a weapon as contained in the BtMG to section 4 (4):

"In contrast to the BtMG, trafficking during which the offender carries a weapon is not punishable according to the AntiDopG. (...) In the BtMG, however, it incurs a penalty of imprisonment for a term of at least five years." (StA 5)

When asked whether there was a practical need for the inclusion of such a penalty enhancing offence, he rather replied in the negative: 
"The largest distribution channels are basically gyms and private sales on the internet. So, I have doubts as to whether there would even be a scope for it." (StA 5)

\section{c) Summary}

The modification and extension of the offences under the AMG in accordance with the BtMG has been widely appreciated. However, there were doubts about the justification of the criminal liability for purchase and possession, especially since the thresholds for significant quantities are apparently so low that they are usually exceeded. This means that mostly selfusers whose criminal intent to pass on doping substances to third parties cannot necessarily be presumed are criminalised. The prohibition of carrying doping substances to or through the territory governed by this act is widely considered to be successful. However, it seems difficult to prove the purpose of doping in these particular cases. Furthermore, the inclusion of penalty enhancing offences pursuant to subsection 4 as well as the inclusion of offences by negligence are deemed appropriate, although negligent conduct rarely becomes relevant in practice.

\section{Findings on procedural issues}

The experts had no files available for their assessment; the evaluation of procedural issues is therefore based on the conducted expert interviews.

\section{a) Initial suspicion}

The justification of an initial suspicion of a criminal offence under section 4 (1) nos. 1 to 3 in conjunction with section 2 naturally depends on the specific criminal act. Nevertheless, there are three typical occasions for grounds of suspicion: the detection of alleged doping substances at customs control and in international postal and goods traffic; investigations against distribution structures and trafficking on the internet, namely the so-called Darknet as well as accidental discoveries, for example during investigations conducted against other offenders. 
In the customs field, most findings are made at the time of import control as well as in mail sorting centres:

"Since we are responsible for Frankfurt Airport and various mail sorting centres, our main focus is on import control. In this context we are investigating by means of physical checks, $x$-rays and checks on documents whether any illegal substances listed in the AntiDopG are imported to Germany. Often, the goods are either wrongly declared or hidden." (Zoll 1)

"Usually, the doping substances are carried by the end consumer, either in his luggage or his car, depending on where the customs controls take place. Otherwise, any doping substances that have been ordered online on the internet or Darknet will be send by post." (Zoll 3)

Added to this are information obtained from investigations into distribution structures and online suppliers of doping substances. Here, for example, investigative approaches arise "if a distribution structure has been eliminated, a server has been mirrored or an underground laboratory has been found. For example, the evaluation of electronic storage media can result in criminal proceedings against the respective customers." (Zoll 3)

A public prosecutor from a specialised public prosecutor's office also confirmed that "(...) especially those lists of online orders or investigations into the Darknet" can lead to further preliminary investigations. (StA 1).

Lastly, "there are also accidental discoveries" (StA 1), thus indications of violations of the AntiDopG given in the course of other investigations:

"We often initiate criminal proceedings due to the fact that we have found various doping substances in the course of a search within a completely different investigation." (StA 1)

With regard to trafficking and manufacturing, grounds for initial suspicion often arise from a consignment if the threshold for a significant quantity is exceeded:

"If illegal substances, medicinal products or active substances are foundwhether being exported to other EU Member States or third countries -, there are grounds for suspicion of trafficking or manufacturing of doping substances in case the threshold for a significant quantity is exceeded." (Zoll 3) 
b) Investigation measures

The expert interviews have shown that investigators and public prosecutors often follow a standardised investigation scheme. This applies, above all, to investigations at customs authorities, which are usually initiated on the basis of similar circumstances. Here, some of the investigation steps are already carried out "onsite" before the public prosecutor's office gets involved:

"Predominantly, we are dealing with seizures of international consignments by post. At first, our colleagues open up the package and assign the medicinal products found to the different areas of law, either to the AMG or the AntiDopG. Next up, it must be calculated whether the threshold for a significant quantity is exceeded. Then, the consignment is seized by the customs officer on site. Afterwards, it is passed on to us for a re-examination and conduct of written hearings in consultation with and on behalf of the public prosecutor's office Frankfurt am Main. Then, the accused shall be given the opportunity to comment on the alleged offence. After he or she has responded, the criminal proceeding is made pending before the public prosecutor's office." (Zoll 2)

In many cases where doping substances are found, the starting points are questionings or hearings in order to investigate the intended use. At the same time, internet research or database queries are carried out in order to check the suspicions or the accused's responses of the written hearing for plausibility:

"In such small cases, we will always conduct written hearings. In Addition, internet research or database queries can be useful." (Zoll 2)

The investigators then decide on further steps in consultation with the prosecution:

"We will conduct investigations by means of written hearing. Anything else that could be relevant to the investigation will be decided in consultation with the prosecution." (Zoll 2)

The search of primate premises was referred to as the most common investigation measure after hearings of the person concerned:

"On a regular basis, we perform searches and secure and seizure doping substances." (Zoll 1) 
"Of course, we carry out a search of private premises at first. We always find something; and if there is an initial suspicion, the chances are even higher." (StA 2)

Other investigation measures were mentioned less frequently and seem to be taken in exceptional cases only:

"We sometimes order telecommunications surveillance in order to track down any customers that are contacting the perpetrator by phone. Besides, we sometimes issue a writ of seizure combined with an attachment order to confiscate the gained profits." (StA 2)

"Due to the fact, that section 4 (4) no. 2 (b) is a serious crime within the meaning of section 100a (2) no. 3 StPO, telecommunications surveillance can be performed. This includes checking telephone and e-mail records, observations or placing listening devices in the car or outside the house." (StA 3)

The possibility of telecommunications surveillance is seen as an improvement to the investigation measures because otherwise it would be difficult to investigate criminal acts of commercial trafficking that take place underground:

"In major proceedings concerning criminal acts on a commercial scale it is advantageous that we can rely on telecommunications surveillance." (Zoll 1)

"With regard to the act of trafficking for commercial gain our possibilities of investigation have certainly improved. And we are happy to rely on it because otherwise underground laboratories are just really hard to detect." (StA 1)

Access to content stored in messenger services was named one of the greatest difficulties when providing evidence:

"Due to the fact that most of the communication takes place via messenger services like WhatsApp, providing the evidence has been more difficult." (Zoll 1)

A further aspect is the communication in restricted online forums and chat rooms that are inaccessible to third parties:

"Furthermore, communication takes place in restricted chat rooms that we have no access to." (Zoll 1) 
Lastly, encrypted or even deleted e-mails were mentioned as an obstacle to the investigations:

"For example, such internet programmes that ensure the immediate deletion of the e-mails after they have been sent are used by the perpetrators." (Zoll 1)

The customs investigators often mentioned that they carry out transnational investigations on a regular basis. It is notable that the cooperation with the authorities of other EU Member States seems to be standardised and apparently runs without any major difficulties:

"If I get an e-mail from Frankfurt Airport telling me that a consignment from China including $50 \mathrm{~kg}$ of testosterone being on its way to France has been seized at Frankfurt Airport, I will immediately inform our French colleagues. Then, they can either take immediate measures or tell us to proceed with delivery to France for further examination on site." (Zoll 1)

"Usually, our findings on the sender, receiver and the quantity of doping substances are passed on to the receiving country in order to find out about the identity of the receiver and to see if they are interested in taking over the proceedings. This is done by our department of international administrative and mutual legal assistance as provided for in the Naples II Convention." (Zoll 3)

However, it appears to be a problem that requests for mutual legal assistance addressed to non-EU States often fails due to the lack of mutual criminal liability when the active substance concerned is released over-thecounter and thus not recognised as a doping substance in the respective State or when there is no comparable ban to section 2:

"Outside the EU, some of the doping substances that are listed in the AntiDopG are not even punishable because they are released over-the-counter." (Zoll 1)

c) Conclusion of the proceedings

The consultation of public prosecutors and judges did not reveal any particularities concerning the conclusion of the proceedings. As with other crimes of minor and medium gravity, many proceedings are terminated. However, unlike with self-doping, summary penalty orders are issued on a 
regular basis (sometimes even predominantly) and - in serious cases - public charges are preferred.

"Actually, quite a lot of the proceedings are terminated in accordance with section 153 StPO." (StA 5)

"The proceedings are predominantly concluded by a summary penalty order and a fine. In case there are huge amounts of doping substances or previous convictions of the perpetrator, public charges may be preferred. But then, the criminal proceedings are usually either terminated in accordance with sections 153 et seq. StPO or the sentence is suspended on probation." (StA 6)

Besides relevant previous convictions, the amount of doping substances found plays a decisive role according to the public prosecutors.

"At first we need to determine by how much the threshold for a significant quantity was exceeded. Then, we will check the accused's criminal record." (StA 5)

Especially if the threshold for a significant quantity has only slightly been exceeded, the investigators will consider a termination of the proceeding. It also becomes clear that the threshold is currently set so low that even the possession of twice the amount of the significant quantity is not considered to be at risk of trafficking.

"For example, the perpetrator has ordered twice the amount of the significant quantity. However, a risk of trafficking in doping substances still seems rather far-fetched to me." (StA 5)

The incorporation of the serious cases referred to in the AMG into the penalty enhancing offences pursuant to section 4 (4) that are punishable by a minimum sentence of imprisonment of one year leads to the fact that criminal proceedings can no longer be terminated in accordance with sections 153 et seq. StPO.

"The main change for us is probably that fewer proceedings are terminated now that the penalty range has been increased." ( $R$ 1)

Nevertheless, the interviewed judges stated that they are only rarely concerned with proceedings pursuant to section 2 in conjunction with section 4. This also applied to the interviewed judge at the specialised court.

Interviewer: "How many of your proceedings are doping-related?" 
$R^{122}$ : "Probably about 5\%, but definitely not more than that."

The judge, who does not work at a specialised court, reported five cases of doping during the last five and a half years that he has been in charge of doping violations.

The criminal proceedings - if a conviction is handed down -are usually concluded by fines; according to the judge, sentences of imprisonment are rarely ordered, only if there are many previous convictions. Here again, in practice there are no recognisable differences from other areas of crime.

\section{d) Characteristics of the court proceeding}

"Many proceedings are carried out in the same way. Usually, the defendant has lodged an objection against the summary penalty order at the court which leads to a main hearing. At the main hearing the defendants often make a confession.

Larger-scale proceedings for trafficking in doping substances are carried out similarly to the ones for trafficking in narcotics. However, in cases of doping, the defendants and their defence counsels are usually not as professional and familiar with the proceedings as the parties to the proceedings under the BtMG." (StA 6)

According to the respondents, the majority of cases conducted before court is focused on bodybuilding or popular sport. Thus, accusations in accordance with section 2 rarely seem to concern competitive sport.

"Typically, the defendants are either bodybuilders or gym owners who pass it on to their customers. Since they are also using the doping substances themselves, it is basically an act of self-harm. But so far there haven't been any convictions of competitive athletes." ( $R$ 2)

With regard to the course of the proceedings, the respondents could not identify any particularities.

"There are no particularities about the court proceedings. So, it's like any other trial. There are no differences." (StA 5)

122 A number is not given here in order to avoid any conclusions on the other statements made by the judge at the specialised court. 
The proceedings were usually pretty simple and were conducted in an expeditious way.

"Well, I'd say the proceedings tend to be rather simple and expeditious. Often, just one main hearing will be enough." (StA 6)

"The proceedings don't take excessively long. They are conducted just as quickly or slowly as any other one." (StA 7)

For the most part, there were no evidentiary problems during the course of the proceedings.

"Usually, the accused does not even apply for evidence to be taken in his defence. I mean, if you find doping substances at his or her house, it is pretty clear. So, we never even had to take any evidence." ( $R$ 2)

For this reason, negotiated agreements are also extremely rare in the main hearing.

"Most proceedings concern cases of petty crime such as possession of doping substances that are only punishable by a fine of a limited number of daily rates. Besides, the doping substances were found so there's clear evidence. So, these kinds of proceedings aren't really suitable for a negotiated agreement." (StA 4)

"No. We don't reach any negotiated agreements in these trials." (StA 6)

However, experts' opinions often have to be obtained in order to "classify and determine the quantity of those doping substances" (StA 3). This may lead to delays - but apparently only in individual cases.

"If the courts insist that every single ampoule or every single medicinal product is checked for its active substance, it will obviously take its time. This may prolong the proceeding. In my opinion, that's not always necessary." (StA 7) 
4. Evaluation

a) General assessment

Opinions in criminal law literature fundamentally criticize the prohibitions of section $2 .{ }^{123}$ Due to the fact that the prohibitions do not presuppose a connection to competitive sport, any sporting activity that is merely a private matter and which does not require compliance with any rules of sports law would also be included. If, however, the use of doping substances would be considered unobjectionable under sports law, doping could not damage the integrity of sport. The prohibitions could be justified by the objective of health protection, but then the provisions would mostly protect people acting on their own responsibility from any selfdamaging acts. Such paternalism within criminal law could hardly be justified. ${ }^{124}$

On the other hand, one could argue that the freedom to use doping substances on one's own responsibility would not yet imply a right to pass on these doping substances. ${ }^{125}$ In this respect, threats to young athletes, for example, who cannot take decisions on self-damaging acts on their own responsibility yet, cannot be ruled out. ${ }^{126}$ However, doubts remain as to the criminal liability considering the implementation of the prohibitions of purchase and possession. In the expert interviews, the respondents also expressed concerns about the criminalisation of purchase and possession of doping substances. A judge and a public prosecutor, along with the critics in literature, questioned whether the offender's protection against selfharming acts would generally be a matter of criminal law:

"I think everyone has to decide on his or her own whether or not to use doping substances." (StA 2)

123 See for the whole issue MüKo-StGB/Freund, 3rd ed. 2018, AntiDopG, $\mathbb{S} 1$ 1-4 Rn. 21.

124 For further details see Freund, FS Rössner, 2015, p. 590; Lehner/Nolte/Putzke/ Rössner, AntiDopG, Vor $\$ 1$ Rn. 25.

125 Weber BtMG/ibid., AntiDopG, $\$ 1$ Rn. 6.

126 BT-Drs. 18/4898, 17; Eising, Die Strafbarkeit des Eigendopings, 2018, p. 86; On the former legal situation in detail: Fiedler, Das Doping minderjähriger Sportler, 2013, p. 26 et seq.; Heger, Strafrechtliche Besonderheiten im Umgang mit minderjährigen Leistungssportlern, in: Kauerhof/Nagel/Zebisch, 2010, p. 25 et seq. 
"If the state was in charge of preventing us from harming ourselves, then he would also have to ban alcohol. I know that's a bit of an exaggeration but still, think about it." ( $R$ 2)

The legislator has tried to circumvent the problem of legitimacy of criminal liability for purchase and possession of doping substances by justifying it with the risk of passing on to others; thereby, both criminal acts therefore constitute offences that include actions prior to dispensing, selling or traffic in doping substances. However, this legitimation approach does not go with the fact that the thresholds for significant quantities are so low that the prohibitions mostly cover mere self-users (see 2.a.cc. above).

The fact that criminal prosecution mainly concerns self-users in the area of bodybuilding is openly criticised by one judge. It would only lead to improvements in criminal prosecution statistics but without changing the actual problem of sports doping:

"If I wanted to increase the numbers and improve the statistics, I could simply order the search of the private premises of several bodybuilders. We will always find something there." $\left(\begin{array}{l}R \\ 2\end{array}\right)$

The easily achieved successes in investigations against self-users in the leisure area and outside of organised sport are contrary to the lack of success in the combat against doping in international competitive sport:

"You get the impression that the government does not take fighting doping serious enough." ( $R$ 2)

\section{b) Legislative implementation}

In contrast to the fundamental criticism of the application of section 2 to self-users in the leisure area, the formulation of the prohibitions under section 2 was otherwise assessed in a predominantly positive way. There was only one decidedly negative opinion in this matter that did not provide any further explanation for his or her critical judgement.

"In my opinion, the Act is insufficient." (R 1)

However, a clear majority of those questioned came to an overall positive conclusion, in particular due to the important symbolism as well as the good practical manageability of the provision. Two public prosecutors em- 
phasized the particular symbolic meaning of the incorporation of the prohibitions laid down in the AMG into the AntiDopG:

"Having an individual AntiDopG definitely underlines the importance." (StA 2)

Another public prosecutor emphasized that it was important to exclude these provisions "from this confusing and messed up AMG". The AntiDopG has "given them a greater meaning (...)". (StA 3)

A second group of respondents appreciated the easy manageability of section 2 as well as the clear comprehensibility of its content. One judge said:

"You can easily implement [the provision] without having to wonder what might have been meant." ( $R$ 2)

When asked to assess section 2, a public prosecutor replied as follows:

"I consider section 2 to be exemplary due to its clarity. Therefore, it is easily manageable." (StA 2)

The customs investigators also came to a positive conclusion due to the comprehensibility and manageability of the provision:

"It is easily comprehensible and you will be able to familiarise yourself with section 2 and the relevant annex to the Act without further problems. More problematic is, however, that the legislator can no longer keep up with the list of doping substances due to the ongoing development of new substances. But with the help of our science centre we manage to work it out." (Zoll 1)

"The introduction of the AntiDopG has made work much easier for us. The provisions are so much clearer and provide more possibilities of investigation." (Zoll 1)

Only one customs investigator said that the AntiDopG would not have brought about any major changes compared to the AMG. His work would have "actually remained the same." (Zoll 2)

c) Suggestions for improvement

aa) Substantive law

Suggestions for improvement in substantive law did not concern the offences under section 2 , but (a) the question whether quantities may be 
added up as well as (b) the formulation of the Doping Substances (Quantities) Regulation (DmMV).

"In most cases, the offender did not just use one doping substance but rather a mixture of various doping substances in order to counteract adverse reactions. For the determination of a significant quantity each substance will be calculated separately. But, if we have three different medicinal products of 0.9 times the significant quantity each, we will add them up to obtain a total amount of 2.7 times the significant quantity. However, it is nowhere written that the quantities may be added up. So, I would appreciate an explicit reference to this either in the AntiDopG, the DmMV or the explanatory memorandum to the Act." (Zoll 3)

The fact that the possibility of adding up quantities intensifies the effect of criminalising self-users in the fitness sector who do not even have the intent to pass on doping substances, has not been discussed by the customs investigator. In addition, one interviewee suggested a more precise formulation of the DmMV, which would "not be entirely clear sometimes":

"We sometimes come across substances where it's just really hard to know if they are even covered by the DmMV and if so which part of the DmMV they belong to. So, it would be nice to specifiy the Regulation." (Zoll 3)

In order to explain, the interviewee referred to a specific request:

"I am just going to read this out: 'We have compiled the following suggestions and questions regarding the amendment of the DmMV:

(1) What exactly are 'other substances related to anabolic-androgenic steroids'? Do, for example, trestolone, epiandrosterone and arimistane belong? When exactly substances are structurally related? Does there have to be scientific evidence that the substance has an anabolic effect? What kind of evidence is needed (animal tests, clinical trials)? Are the so-called 'prohormones' also meant here?

(2) In general, it would be extremely helpful if all the CAS Registry Numbers of the mentioned substances were given. This is the only way to reliably assign the names (for some of them there are plenty of synonyms) to the respective chemical structure. As an example: When searching for methyl nortestosterone on the internet, the following substances will come up: $11 \beta-M e-$

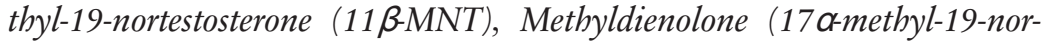
ס9-testosterone), Metribolone (methyltrienolone; R-1881; 17a-methyl-19nor- $\delta^{9,11}$-testosterone), Normethandrone (methylestrenolone; normethisterone; 17a-methyl-19-nortestosterone) or Trestolone (7a-methyl-19-nortestosterone; MENT). 
(3) It would be helpful if some examples of selective androgen receptor modulators were mentioned (LGD-4033, RAD 140, enobosarm, andarine, YK-11,...).

(4) How should the substance SR9009 be evaluated?

(5) For peptides: There are a few peptides (e.g. GHRP6 and pralmorelin) with modified versions due to a different terminally attached amino acid (so far: glycine). Are these also included in the 'Growth Hormone Releasing Peptides'?'” (Zoll 3)

bb) Procedural law and investigatory powers

With regard to investigation methods, two points were raised: the introduction of a leniency comparable to the BtMG and an extension of section 100a StPO in order to include further modi operandi.

"The introduction of a leniency analogous to section 31 BtMG would be important to us. Because in drug-related cases section 31 BtMG comes into effect on a regular basis when large amounts of narcotics are involved. There is nothing like it in the AntiDopG so it would be a great advantage to introduce something similar." (Zoll 1)

A public prosecutor also suggested to extend section 100a (2) StPO:

"One could think about widening the scope of section 100a StPO by including section 4 (4) as a whole. That would be a very reasonable consideration perhaps." (StA 1)

Several times, the investigators addressed the need for greater coordination and collaboration of investigations as well as communication. Particularly with regard to lifestyle drugs that - according to criminalistic experience are often used by middle-aged or older people for other purposes than doping in sport, one investigator expressed the wish for better pre-structuring of the proceedings and the conduct of fewer time-consuming and often even unsuccessful investigations:

"Our experience has shown that certain medicinal products usually cannot be associated with criminal acts in accordance with the AntiDopG. And this can be communicated with other investigating authorities. Besides, one could think about cutting these proceedings short when certain indicators are given." (Zoll 2) 
The question of who should be responsible for the seized doping substances was also of practical importance. One interviewee said that "the main problem is that it hasn't yet been clarified who is responsible for destroying the doping substances after seizure, how and at whose expenses it should be done. Right now, every case is treated differently." (Zoll 3)

Lastly, when asked about improvements, a customs officer referred to the lack of resources:

"In terms of resources, we are short on staff. However, communication and cooperation with our international colleagues works perfectly." (Zoll 1)

\section{Overarching aspects}

In the following, the findings of the evaluation study that concern both of the previously examined provisions (sections 2 to 4 ) or go beyond the issues that are directly related to the substantive provisions will be presented.

1. Sentencing range and limitation period

The sentencing ranges for sections 2 and 3 are laid down in the common provision of section 4 . The limitation period is based on the maximum set out in section 4 . The issues related to section 4 shall therefore be discussed jointly.

a) Sentencing range contained in section 4

Infringements of sections 2 and 3 are generally punishable with up to three years' imprisonment or a fine (section 4 (1)). In this respect, the legislator has decided upon a consistent scheme of sanctions, although the criminal offences concerning self-doping do not contain the legally protected right to public health, but rather the legally protected right to integrity of organised sport and its fundamental ethical and moral values such as fairness 


\section{B. Results of the evaluation}

and equal chances. ${ }^{127}$ In view of the fact that the legal prohibition of selfdoping has been particularly controversial, it is - seen from a criminal policy perspective - understandable that the legislator does not criminalise self-doping more harshly than infringements of section 2 .

However, the consistent scheme of sanctions is disrupted at two points:

On the one hand, the penalty enhancing offences of section 4 (4) solely refer to infringements of section 2 (no. 1 applies to all criminal acts of section 2, no. 2 only applies to section 2 (1) and (2)). Here, a penalty of imprisonment of one to ten years may be incurred; the criminal acts are therefore serious criminal offences. ${ }^{128}$ This provision is not only relevant to the sentencing, but also to the admissibility of telecommunications surveillance since it is restricted to cases of section 4 (4) no. 2 (b) as referred to in section 100a (2) no. $3 \mathrm{StPO}$, as well as to the leniency contained in section $46 \mathrm{~b}$ StGB that only applies to the penalty enhancing offences of section 4 (4).

On the other hand, the threatened penalties for the criminal liability for purchase and possession differ from each other. The purchase or possession of significant quantities of a doping substance in accordance with section 2 (3) shall be punishable with up to three years' imprisonment (section 4 (1)). However, anyone who purchases or possesses a doping substance with the aim of administering it or having it administered to oneself without medical justification in order to gain an advantage in a competition of organised sport shall be punishable with up to two years' imprisonment (section $4(2)$ ).

If the perpetrator acts negligently (in the cases covered by section 2 (1)) the penalty shall be imprisonment for a period of not more than one year or a fine, section $4(6)$.

127 BT-Drs. 18/4898, p. 18; Momsen, KriPoZ 2018, 21, 22; Graf/Jäger/Wittig/Eschelbach, Wirtschafts- und Steuerstrafrecht, 2nd ed. 2017, AntiDopG, $\mathbb{} 4$ Rn. 1. Cf. Kubiciel, KriPoZ 2018, 21 seq.

128 However, section 4 (5) provides for the possibility of a less serious case; the penalty under subsection 4 shall then be reduced to imprisonment for a period of three months to five years. 
aa) Increasing the maximum sentence contained in section 4 (1)

Some of the experts from law enforcement ${ }^{129}$ were in favour of increasing the maximum sentence contained in section 4 (1) up to five years. This was justified by the fact that the previous sentencing would suggest that the handling of doping substances is a petty crime.

"The maximum sentence contained in section 4 (1) is far too low. I would call for a maximum sentence of imprisonment of five years. Because it is contradictory to emphasize the importance of the prosecution of offenders under the AntiDopG on the one hand but to punish these offences like a petty crime on the other hand. So, we need to increase the maximum sentence." (StA 3)

The respondents also pointed out that the unlawful handling of doping substances would be comparable to crime involving narcotic drugs. By increasing the maximum sentence, the AntiDopG could get in line with the provisions of the BtMG. ${ }^{130}$

"The maximum sentence contained in section 4 (1) AntiDopG is only three years whereas the maximum sentence contained in section 29 (1) BtMG is five years in fact. So, increasing the maximum sentence up to five years would certainly be appreciated." (StA 1)

Other interviewees from law enforcement, however, considered the sentencing range to be appropriate and could not see any need for change. The athletes' representatives were also against an increase of the sentencing range.

"The sentencing range is average. And only in rare cases the maximum sentence of imprisonment of three years will even be ordered." ( $R$ 2)

"Well, the sentencing range is a bit lower than others. But I think it's sufficient. It leaves enough room for appropriate sentences in individual cases." (StA 6)

"I'm not in favor of increasing the sentences." (A 2)

129 Three of the prosecutors considered the increase to be reasonable, four prosecutors - as well as the judges - saw no need for change.

130 One expert also mentioned that the AntiDopG does not provide for trafficking during which the offender carries a weapon as contained in section 30a BtMG in the form of a penalty enhancing offence. 
It has also been pointed out that an increase of the sentencing range would not have a deterrent effect on athletes. Enhancing the effectiveness of criminal prosecution and creating a real risk of detection would be much more decisive. ${ }^{131}$

"I don't think that increasing the sentencing range would have such a big impact on athletes. Any violations against the AntiDopG already have farreaching consequences for them, e.g. being excluded from sport, being disrespected, losing their livelihood. (...) So, it would be more efficient to just increase the risk of detection than increasing the actual sentencing range." (A 1)

bb) Inconsistency of the applicable sentencing ranges?

Some interviewees referred to a possible inconsistency in the determination of sentencing ranges for the criminal liability for purchase and possession. As explained above, the purchase or possession of significant quantities of a doping substance in accordance with section 2 (3) shall be punishable with up to three years' imprisonment whereas purchase or possession of doping substances for the purpose of self-doping in accordance with section 3 (4) shall only be punishable with up to two years' imprisonment. This discrepancy in the maximum sentences was sometimes considered to be an unnecessary privileged treatment of athletes.

"I don't understand why competitive athletes shall only be punishable with up to two years imprisonment whereas recreational athletes shall be punishable with up to three years' imprisonment. It seems like athletes are having privileges. But there is no reason for it. Especially with respect to their significance in sport, competitive athletes should be prosecuted just like any other recreational athlete." (StA 3)

"It's contradictory that the competitive athlete's possession of doping substances will not be punished as severely as the recreational athlete's possession of doping substances. I don't think that's appropriate." (StA 2)

Other respondents disagreed with the criticism by referring to the fact that the offences demand different prerequisites for criminal liability.

131 See also Eising, Die Strafbarkeit des Eigendopings, 2018, p. 165 et seq. 
"It's actually the opposite of privileged treatment because recreational athletes shall only be held criminally liable for the possession of a significant quantity of doping substances whereas competitive athletes shall always be held criminally liable for the possession of doping substances no matter what." (StA 7)

\section{b) Limitation period}

The general limitation period of five years contained in section 78 (3) no. 4 StPO applies to the criminal offences pursuant to section 4 (1) and (2). If the perpetrator meets the conditions of the penalty enhancing offences under section 4 (4), the limitation period is ten years in accordance with section 78 (3) no. 3 StPO. According to section 78 (4) StPO, this applies regardless of whether there are any less serious cases pursuant to section 4 (5). The limitation period is three years in the case of negligence pursuant to section 4 (6) as referred to in section 78 (3) no. 5 StPO.

When analysing the files, issues regarding the limitation period did not occur. This is probably also due to the fact that the AntiDopG and with it the offence of self-doping has entered into force in December 2015, thus only less than five years ago.

With regard to the limitation periods, the respondents did not see any need for change.

"So far, there haven't been any problems with the limitation periods. I have no requests for change." (StA 4)

Increasing the limitation period for penalty enhancing offences pursuant to section 4 (4) up to ten years compared with a former limitation period of five years contained in the previous provision under section 95 AMG was appreciated by the interviewees. This is where the fact that the provision is no longer formulated as an example of especially serious cases, but as a serious criminal offence comes into play.

"Due to the fact that the limitation period has only been five years under the $A M G$, many doping-related crimes have gone unpunished - just think of the German physician carrying out blood doping since 2011 ("Operation Aderlass") or the Olympic Games in Sochi in February 2014. These criminal of- 
fences could have been prosecuted if there had already been a limitation period of 10 years back then." 132

c) Assessment

aa) Maximum sentence

There is no reason to believe that a maximum sentence of three years will lead to a relativization of the wrongfulness contained in the AntiDopG. A maximum sentence of three years does not only apply to typical cases of "petty crime", but also to handling stolen data (section 202d StGB) or dissemination, procurement and possession of youth pornography (section $184 \mathrm{c} \mathrm{StGB}$ ) or incitement of masses (section 130 (2) StGB). Increasing the maximum sentence would also have no practical significance: On the one hand, it is common practice in German Courts - as it is pointed out by the respondent $R$ 2-that the penalties rather approach the respective minimum sentence than the maximum sentence. On the other hand, the empirical study has shown that almost all of the proceedings for self-doping are terminated without further consequences. Only three of the evaluated proceedings for self-doping have been concluded by summary penalty orders; in two cases fines of 30 or 40 daily rates have been imposed bearing in mind the minimum of five and the maximum of 360 daily rates in accordance with section 40 (1) StGB. Therefore, it cannot be said that the applicable sentencing ranges would not allow for a sentence that meets the degree of the offender's guilt in consideration of the severity of the offence committed. From this perspective, the discussion on increasing the maximum sentences currently has no significance in legal practice. Apart from this, there is reason to doubt whether an increase of the maximum sentences would have a greatly increased general and special preventive effect. ${ }^{133}$

132 Due to the concrete reference to the proceeding "Operation Aderlass", a number is not given here in order to avoid any conclusions on the other statements made by the public prosecutor.

133 It is recognised in criminology and criminal justice theory that preventive effects are not so much achieved due to the threatened penalty and its severity but rather due to the probability of detection and sanctioning and the rapidity of the State's reaction to the crime. See Eisenberg/Kölbel, Kriminologie, 7th ed. 2017, $\$ 41$ Rn. 14,22 et seq., $\$ 42$ Rn. 5 et seq. 
Nevertheless, the legislator is of course free to adjust the sentencing ranges of the AntiDopG to the ones of the BtMG for symbolic reasons. However, such an alignment is not mandatory, also due to systematic reasons; the AntiDopG and the BtMG do resemble each other in terms of structure and formulations, but pursue different protective purposes. In addition, narcotics cause considerably greater harm to individuals and society than doping substances.

bb) Criminal liability for purchase and possession

The criminal offences pursuant to sections 2 (3) and 3 (4) do not only differ in terms of addressees, but primarily in terms of their scope of protection. According to section 2 (3) the purchase and possession of significant quantities shall be punishable as it is an offence that includes actions prior to trafficking. ${ }^{134}$ In contrast, according to section 3 (4) the purchase and possession of insignificant quantities of doping substances shall be punishable because it is an offence that includes actions prior to self-doping. Due to the fact that section 4 (1) provides for the same sentencing range for trafficking and self-doping, it could be well justified - in terms of systematics - to also align the threatened penalties for the respective offences that include actions prior to the crime. In this case, however, it would be consistent to decrease the maximum sentence under section 2 (3) in order to take into account the less extensive wrongfulness of an offence that only includes actions prior to crime. Whether this is advisable in terms of legal policy is up to the legislator.

cc) Limitation period

Currently, there is no reason to change the limitation periods.

2. Establishment of specialised public prosecutor's offices and courts

Currently, there are specialised public prosecutor's offices in Freiburg, Munich and Zweibrücken focusing on combatting doping; in other federal

134 BT-Drs. 18/4898, p. 25; Körner/Patzak/Volkmer/Volkmer, BtMG, 9th ed. 2019, AntiDopG, $\mathbb{} 4$ Rn. 42. 
States - for example in Hesse - their establishment is being discussed. A specialised court for doping has only been established in Zweibrücken (Rhineland-Palatinate) so far.

a) The demand of the NADA and the athlete's associations

In various opinions, the NADA and the athlete's associations have called for the establishment of further specialised public prosecutor's offices and courts. The reason given for the proposal is the complexity of the matter, which would require a particular specialisation, especially of the investigating officers.

"Lastly, the NADA calls for the establishment of further specialised public prosecutor's offices for the purpose of fighting against violations of the AntiDopG. Only those trained and specialised investigators are able to work quickly, efficiently and well-focused. The good cooperation between the specialised public prosecutor's office Munich I and the Austrian investigators serves as a positive example." 135

"In order to detect similar cases in future, we need sufficient capacities and expertise of the investigating authorities. We therefore consider it useful to establish additional specialised public prosecutor's offices for the purpose of combatting doping." 136

During the interviews, the experts from the NADA and the athletes associations re-emphasised their wish for greater specialisation of the judiciary. The experts reported negative experiences with public prosecutor's offices and courts that had little expertise in handling doping offences.

"If we're lucky we're working with one of the few public prosecutors who are interested in this matter and have at least a bit of experience from a previous case. But mostly it is a matter of coincidence which public prosecutor you are working with and if he or she has got any experience at all." $(N)$

135 NADA, public hearing of the Sports Committee of the German Bundestag, Need for Amendments and Supplements to the Act against doping in sport (AntiDopG), p. 5.

136 Athleten Deutschland e.V., hearing of the Sports Committee, 23.10.2019, p. 3. 
The interviewees traced the frequent termination of proceedings particularly back to the fact that public prosecutors are not familiar with the AntiDopG and the practical challenges of investigations in the field of doping in sport.

"In general, you get the impression that the ordinary public prosecutor's offices are just way too unfamiliar with these very specific issues and that the proceedings are therefore terminated very, very quickly. (...) This is probably due to the fact that these cases do require certain knowledge of how to properly investigate doping offences." (A 3)

However, the respondents have made positive experiences with the specialised public prosecutor's offices.

"The specialised public prosecutor's offices in Munich, Freiburg and Zweibrücken are well-organised in order to act quickly, professionally and focused. (...) Therefore, cases like the ones that just happened in Erfurt, Munich or Seefeld for example can quickly be dealt with." $(N)$

b) On the establishment of specialised public prosecutor's offices

aa) The perspective of the judiciary

The interviewed public prosecutors were predominantly in favour of the establishment of specialised public prosecutor's offices. They shared the associations' assessment that an effective implementation of the AntiDopG would require special knowledge, not only in terms of law but also in terms of sports medicine.

"The establishment of specialised public prosecutor's offices seems very reasonable to me. (...) Proceedings under the AntiDopG require a lot of special knowledge, e.g. regarding section 4 (7) or the determination of significant quantities in accordance with section 4 (1) no. 3. (...) Bearing that in mind, we would wish for even more specialised public prosecutor's offices." (StA 1)

"I think that a specialisation of public prosecutors is necessary because they do not only need to know about criminal law, but also about medicine, pharmacy, anatomy or chemistry. They need to know how these medicinal products affect the human body or how they are manufactured. So, these doping cases are just too complicated and too complex to not be dealt with exclusively. Therefore, I consider specialised public prosecutor's offices absolutely necessary." (StA 3) 
Even those public prosecutors who did not work at specialised public prosecutor's offices considered a concentration of proceedings to be useful.

"The establishment of specialised public prosecutor's offices seems reasonable to me in order to pool knowledge on the subject of doping and be able to prosecute competitive athletes on a larger scale." (StA 2)

Besides greater knowledge on this subject, the fact that public prosecutors would have a better overview of the doping scene within their federal State was seen as an advantage of the specialised public prosecutor's offices.

"The advantages of the specialised public prosecutor's offices include, above all, the specialisation and the possibility to properly address the doping scene within the respective federal State. Because if you start looking for it, you will find a lot of doping cases. In 2009, when we started, we only had 170 cases and last year we have been dealing with 1500 cases." (StA 3)

Furthermore, the public prosecutors at specialised public prosecutor's offices are able to establish fixed ways of communication with their contacts at customs authorities or the NADA, for example.

"The reason given for the establishment of specialised public prosecutor's offices is the need for special knowledge and better cooperation between the public prosecutors and the customs authorities, the police and the NADA. It certainly is an advantage that the cooperation with the customs authorities, the police or the NADA becomes a matter of routine." (StA 6)

Other interviewees did not consider the establishment of specialised public prosecutor's offices to be mandatory. One respondent believed that a specialisation would be advantageous to every area of law and that the AntiDopG would not have any fundamental particularities in this respect.

"I mean, the specialised public prosecutor's offices obviously have the advantage of specialized staff that can run such proceedings more easily and quickly. But this would also be advantageous to every other area of law such as the BtMG or sexual offences. So, I am neither for nor against it." (StA 5)

Another public prosecutor pointed out that the concentration of proceedings under the AntiDopG could also lead to difficulties when conducting the proceedings; for example, if besides the alleged offence of unlawful handling of doping substances suspicions of drug offences need to considered.

"But there are also disadvantages. In our experience, proceedings that are somehow connected are often split up due to this distribution of responsibili- 
ties. If, for example, doping substances as well as narcotics are found during a search of the offender's private premises, the proceedings under the BtMG will be conducted in Koblenz and the proceedings under the AntiDopG will be handed over to us. But then, before we can even start investigating, we need to sort out whether these two proceedings should be run together or on their own and who should run them. That's always a bit of a problem." $\left(S t A^{137}\right)$

Only one of the respondents (who did not work in a specialised public prosecutor's office) was against a concentration of competence. It would make sense for high-profile cases in competitive sport, but not for the majority of cases in the field of bodybuilding or popular sport.

"The establishment of specialised public prosecutor's offices would only make sense if they were investigating crimes in competitive sport. That would not only include the competitive athletes themselves, but also their support personnel. However, a concentration of all the proceedings under the AntiDopG, including cases in the field of popular sport like bodybuilding, would simply lead to a waste of resources." (StA ${ }^{138)}$

bb) Findings from the evaluation of files

The evaluation of files- prudently - supports the assessment that specialised public prosecutor's offices can provide more effective criminal prosecution due to established structures and greater experience of handling doping offences. However, the findings from the evaluation of files should not be overestimated here as they are limited to cases of self-doping.

Two of three summary penalty orders were issued by the specialised public prosecutor's office in Freiburg; they also provided most of the proceedings for self-doping for the evaluation. In contrast, only one relevant proceeding was made available by the specialised public prosecutor's office in Zweibrücken; the proceeding was conducted thoroughly and by means

137 Due to the concrete reference to the federal State, a number is not given here in order to avoid any conclusions on the other statements made by the public prosecutor.

138 Due to the concrete reference to the federal State, a number is not given here in order to avoid any conclusions on the other statements made by the public prosecutor. 


\section{B. Results of the evaluation}

of various investigation measures though. The fact that there were concrete allegations rather than just a general reference to the AntiDopG as otherwise frequently found (see p. 12 above) applies to all of the specialised public prosecutor's offices. Communication between the specialised public prosecutor's offices and the NADA or the customs authorities ran smoothly, too. The specialised public prosecutor's offices have terminated the proceedings less frequently in accordance with section $153 \mathrm{StPO}$ and more often in accordance with section 170 (2) StPO. This can be taken as an indication that the elements were reviewed more intensively and that the proceedings were not simply terminated due to considerations of practical expediency in case of investigative difficulties.

c) On the establishment of specialised courts

With regard to the establishment of specialised courts, the interviews revealed a differentiated picture. While the representative of the NADA fully endorsed specialised courts, only two of the interviewed experts from the judiciary were clearly in favour of a centralised jurisdiction of the courts. The remaining respondents saw advantages and disadvantages that largely balanced each other.

"Specialised courts are certainly a mixed blessing." (StA 1)

First of all, the benefit of uniform case law within each federal State for the interpretation of the AntiDopG was discussed. While some considered a consistent interpretation of law to be useful, others saw a danger of the consolidation of the case law on a regional level.

"One of the disadvantages could be the consolidation of the case law. But then again, a quick development of established case law can also be advantageous." (StA 4)

Considerations of practicability were also of importance. Some of the interviewed public prosecutors saw the establishment of specialised courts as a chance to avoid time-consuming travelling to different courts throughout the federal State.

"It would have the positive effect that we can avoid external sessional services. Then we would not have to travel so much." (StA 1) 
It was recognised, however, that a centralised jurisdiction of the courts would mean considerable effort by the accused or the summoned witnesses on the other hand.

"It's an advantage for us. We don't have to travel a long way just in order to get to the sessional service. But then of course, the accused or other witnesses need to travel to Zweibrücken. So, there are pros and cons." (StA 5)

A public prosecutor from Zweibrücken, who had made experiences with the specialised court, also pointed out this aspect.

"There is one disadvantage: If we conduct court proceedings from all over Rhineland-Palatinate right here, all the witnesses, police officers, attorneys and the accused himself need to travel to Zweibrücken. Whereas, if we would maintain the ordinary jurisdiction of the courts, the public prosecutor will be the only one to travel around in order to represent the prosecution at court. So, it has its advantages and disadvantages." (StA $\left.{ }^{139}\right)$

However, most respondents believed that a centralised jurisdiction would be useful in terms of expertise.

"We are calling for specialised public prosecutor's offices and courts, because we see a lot of county courts that are struggling to conduct proceedings concerning such a specific area of law besides their daily business. Proceedings under the AntiDopG depend on testimonies and the expert's assessment. Due to the fact that this all new to the courts, one should consider establishing specialised courts." $(N)$

A public prosecutor from the specialised public prosecutor's office in $\mathrm{Mu}$ nich reported that he and his colleagues often had to assist the courts in dealing with the largely unknown offences under the AntiDopG. He therefore suggested to establish specialised courts, at least in the respective districts of Higher Regional Courts.

"Specialised courts do make sense because county court judges just do not have enough experience or knowledge on this subject. To start with, they are usually not familiar with the wording of the law or the conduction of proceedings under the AntiDopG. And that's what we need to help them with. So, a certain specialisation of the courts would be appreciated. I would prefer the establishment of specialised courts within the three districts of the Bavarian

139 Due to the concrete reference to the federal State, a number is not given here in order to avoid any conclusions on the other statements made by the public prosecutor. 
Higher Regional Courts instead of just one specialised court for Bavaria as a whole in order to consider the local peculiarities of the respective doping scene." $\left(\operatorname{StA}{ }^{140}\right)$

A public prosecutor from Zweibrücken praised the judges' work at the specialised court and saw clear advantages for a centralised jurisdiction in terms of expertise.

"Well, the judges who are now concerned with this matter are real experts in their field. Everything runs smoothly. So, in terms of expertise the concentration of proceedings at specialised public prosecutor's offices and courts would certainly be advantageous." (StA $\left.{ }^{141}\right)$

The interviewed judges were divided on this matter. While one of the judges was in favour of establishing specialised courts due to the complexity of the subject, the other judge considered the legal difficulties to be manageable and centralised jurisdiction of the courts to be unnecessary.

"The establishment of specialised courts does make sense because the AntiDopG is very complex and difficult to understand at first reading." ( $R$ 1)

"Well, I don't think that's necessary. Similar proceedings under the BtMG are not centralised either (...) And it's nothing new that we have to familiarise ourselves with uncommon areas of law. I mean, right now I have to deal with a violation of the Animal Welfare Act. So please." ( $R$ 2)

\section{d) Assessment}

The criminal prosecution of doping violations challenges the investigating authorities in several new ways. The AntiDopG is specialised criminal law and regulates the subject of doping that most public prosecutors had little to do within their training or in later practice. The implementation of the AntiDopG requires knowledge of various sports, their organisation, their competitions and compensation structures as well as the areas of application and effects of performance-enhancing substances in order to evaluate

140 Due to the concrete reference to the federal State, a number is not given here in order to avoid any conclusions on the other statements made by the public prosecutor.

141 Due to the concrete reference to the federal State, a number is not given here in order to avoid any conclusions on the other statements made by the public prosecutor. 
the detection of illicit substances in doping samples as well as the experts' opinions. The evaluation of files has shown that this lack of practical experience with doping cases constitutes ground for the termination of proceedings. Investigators who have rarely dealt with doping cases before showed uncertainty in handling the accused's denial for instance and greater reluctance to obtain an expert opinion.

In order to prosecute violations of the AntiDopG more effectively, the establishment of specialised public prosecutor's offices seems to be reasonable. The responsible public prosecutors can acquire the necessary knowledge in terms of law and sports medicine and establish long-lasting contacts with the NADA, athletes' associations and experts. The advantage of centralised jurisdiction not only lies in the concentration of competence and structures, but also in a stronger identification with the subject and the associated responsibility towards the punishment of doping offences. It is unlikely that the specialised public prosecutor's offices will terminate the proceedings due to an aversion to unknown areas of law or simply due to considerations of practicability ${ }^{142}$.

In order to address the justified criticism of splitting up coherent cases into two proceedings under the AntiDopG and the BtMG for example, determining the specialised public prosecutor's offices' jurisdiction in somehow connected proceedings would be useful.

With regard to the establishment of specialised courts, the evaluation has shown an ambivalent picture. The particular professional expertise that goes along with centralised jurisdiction and the frequent handling of similar cases speaks in favour of specialised courts - just as much as it does for specialised public prosecutor's offices. However, a specialisation of courts seems less urgent: the judges will only receive the few cases that are brought to trial, so that they should - particularly on the basis of the public prosecutors' groundwork-be able to familiarise themselves with the subject without any problems. Whether a specialised court should be established, therefore largely depends on the particularities of the respective federal State; that is why the experts do not make a clear recommendation here.

142 StA 6: "Sometimes, the proceedings are terminated due to the fact, that the investigations are just too complex and you don't really know where and how to proceed with the investigations. And I don't think it is worthwhile to investigate even more deeply, because in the end a comparably mild sentence will be imposed. Of course, these are also considerations of practicability. I mean, we always have to ask ourselves: Is it worthwhile to invest so much work?" 
3. Cooperation with the NADA and section 8

According to section 8, courts and public prosecutor's offices may, ex officio, transmit personal data from criminal proceedings to the NADA if the transmitting body considers it necessary to take disciplinary measures in the framework of the doping control system, unless the data subject has a legitimate interest in not having the data. By including this provision, the legislator intended to support the NADA in conducting disciplinary proceedings; the investigation results of the prosecution authorities should be provided to the NADA for the sanctioning of athletes and athlete support personnel. ${ }^{143}$

According to the findings of this study, cooperation between the NADA and the public prosecutor's offices is of particular importance to the prosecution authorities: in cases of self-doping, the NADA is usually the one to report the offence or to inform about positive doping samples and other anomalies. Furthermore, the NADA has often acted as a contact point for questions regarding the type of doping substance or whether the athlete is included in a Registered Testing Pool.

"We can always contact the NADA if we need to know whether the athlete concerned is included in a Registered Testing Pool, for example." (StA 5)

"I believe communication is very important because the NADA obviously has the relevant know-how regarding the structure of certain substances or whether the athlete is included in a Registered Testing Pool, for example. That often needs to be checked." (StA 2)

Respondents from the specialised public prosecutor's offices, who regularly share their experiences with the representatives of the NADA, saw an advantage in these existing, sometimes even informal contacts.

"It's good that we know each other. We are in regular contact by telephone or by mail and meet each other at conferences. And this is really useful. Because if you have already met someone in person, you will be less hesitant to call or e-mail them." (StA 6)

While the NADA often provides important information to the public prosecutor's offices, the reverse flow of information is generally less extensive and less important. Cooperation in the actual sense of section 8 therefore

143 BT-Drs. 18/4898, p. 35. 
rarely took place. Some public prosecutors were not aware of the provision in detail.

"To be honest, I don't really know what's contained in section 8. I'll take another look." (StA 7)

An established practice to provide the NADA with the investigation results for their disciplinary proceedings could not be found in most public prosecutors' offices. Considering the small number of cases that have been relevant so far, this finding should not be overestimated though.

"I'm not so sure whether I have made use of the transmission of data as referred to in section 8 so far. If our investigation results are relevant for the proceeding under sports law, we will of course provide them with our findings if legally permissible. But I can't remember ever having done that." (StA 4)

When the proceeding was initiated on the basis of evidence submitted by the NADA, the public prosecutor's closure statement was - as it appears from the case-files - often sent to the NADA.

"We provide them with our closure statement. Due to the fact that these aspects of criminal law and sports law are so closely linked, the NADA is relying on our investigation results. And they do have a right to receive that information." (StA 7)

However, two public prosecutors from specialised public prosecutor's offices referred to a closer cooperation. The public prosecutors have often asked the NADA not to directly inform the athlete of the positive doping sample in order not to jeopardize the success of investigation measures like a search of private premises for example. In return, the NADA was informed of the investigation results.

"Usually, the NADA reports an offence and starts to conduct their own proceeding under sports law. And this is where we would ask the NADA not to inform the athlete until we have taken our investigation measures. In case of an alleged possession or purchase of doping substances, we will conduct a search of the private premises. And of course, the NADA will reach out to us in order to gain all the information on our investigations. What did the investigations reveal? How did the athlete react to the accusations? And the NADA will certainly use these findings for their own proceeding. So, section 8 allows us to grant inspection of files and to exchange information at an early stage. We practically exchange findings throughout the whole procedure and not only after the proceeding has already been concluded." (StA 3) 
Even though the interviewees themselves had little or no experience with section 8 , they considered the provision useful. No amendments were proposed.

"I would consider the provision sufficient. I think it is very useful because it provides a legal basis for this cooperation." (StA 1)

"Clearly defining the role of the NADA has been a big step forward. I don't see any problems and I couldn't think of anything I would change." (StA 2)

This positive assessment was also shared by the athletes' representatives.

"The transmission of data between the NADA, the courts and the public prosecutor's offices has always been a problem due to the lack of a legal basis. The NADA has always shared their findings, but the public prosecutor's offices, on the other hand, weren't permitted to transmit their data. The fact that this is now regulated by the AntiDopG simply shows the need for cooperation." (A 3)

The opinion of the NADA calls for the extension of the scope of section 8 in order to include all "competent national and international Anti-Doping Organisations". The "international ties between the NADA and the AntiDoping Organisations of other countries as well as the WADA" would require "an advanced exchange of information on an international level". This demand was confirmed in the interview.

"We had evidence, submitted from North America, that a coach intended to enter Europe, in particular Germany, with doping substances. We were able to inform the customs authorities who could then seizure the accused's doping substances right at the airport. But there was one problem: he was not a German citizen. So, even though he may have made himself punishable under German law in accordance with the AntiDopG, the proceeding under sports law was much more complicated. The NADA could not proceed against him because his case was not within their competence. And there was no legal basis for the inspection of files at foreign Anti-Doping Organisations. So, this example has shown that international exchange must be strengthened." $(N)$

\section{Training}

The interviewees were also asked if there has been any training on the subject of the AntiDopG after its entry into force and who was performing it. 
While the public prosecutors and judges reported no systematic training, the customs authorities seem to have developed more training activities:

"Yes, we have performed training, in particular the Customs Criminal Office in cooperation with my predecessor. He designed a nationwide training concept for customs investigators to introduce the subject of doping even before the AntiDopG has entered into force. That happened to be a huge advantage compared to the police." (Zoll 1)

"Yes, I attended training that was provided by a leading colleague in this field. He was mainly involved in customs investigations so be put a focus on that. And when the AntiDopG has entered into force, I asked him to carry out training for all our airport staff that is concerned with the matter." (Zoll 2)

"We actually perform training for the customs investigation service ourselves. And we obviously include the AntiDopG." (Zoll 3)

A representative of the Federal Criminal Police Office did not report any training, but an exchange of information and experience (nationally as well as internationally):

"We also provide political consulting and we participate in conferences once a year where specialist services from all over Germany including the NADA meets up in order to exchange information." (BKA)

In terms of training, the sports federations primarily work with the NADA. It could not be established through the interviews to what extent this training was carried out and if it had a lasting impact. Nevertheless, one federation representative reported that the subjects of integrity in sport and doping should be given even more importance in the future:

"We definitely want to take this issue forward. In the past, we used to perform training for the anti-doping commissioners of our member organisations. But right now, this is mostly done by the NADA in consultation with us. However, we want to make sure to rebuild closer relationships with our member organisations again and we want to provide our assistance." (D)

Especially due to the fact that the AntiDopG is a rather recent law that involves complex issues of law and sports medicine, training is important in order to give the fullest practical effect to its provisions. Training as provided by the customs authorities is therefore just as useful as training in the federations and training for athletes and their environment (coaches, support personnel and physicians). This is not only useful in order to elimi- 


\section{B. Results of the evaluation}

nate occasional information deficits (e.g. regarding the party to whom the standard is addressed to), but above all to enable a cultural change and to firmly anchor the significance of the doping ban in individual and institutional awareness. 


\section{Final opinion of the experts}

\section{General assessment}

The prohibition of self-doping (section 3 in conjunction with section 4 (1) nos. 4 to 5 and (2)) represents the major substantive amendment of the AntiDopG. Besides, the legislator has incorporated the criminal offences under the AMG into the AntiDopG in modified and extended form (see section 2 in conjunction with section 4 (1) nos. 1 to 3). According to section 1 , the purpose of fighting the use of doping substances and doping methods in sport is to protect the health of athletes, to ensure fairness and equal chances in sport competitions and thereby preserve the integrity of sport. These objectives are achieved to different extents. The evaluation has shown that the AntiDopG, in practice, primarily protects the health of recreational athletes (from self-damaging acts) rather than the integrity of sport.

\section{Self-doping (section 3)}

Proceedings for self-doping in the field of competitive sport represent a comparatively small proportion of the preliminary investigations. Most of them are terminated and only in very rare cases they are concluded by a summary penalty order; the inspected files that were provided by the public prosecutor's offices did not once indicate that there was a conviction.

The fact that only few of the preliminary investigations concern violations of the ban on self-doping is not so much due to the formulation of sections 3 and 4 . More decisive is that the prosecution authorities rarely receive incriminating information on cases of self-doping. None of the investigated proceedings were initiated following a report made by a federation or sports club. Usually, criminal proceedings are initiated following a report made by the NADA or on the basis of information about positive doping samples as provided by the NADA. These naturally concern individual athletes rather than structures and networks.

Among the investigating authorities there is still some uncertainty in handling cases of self-doping. Regarding the subjective element, some public prosecutor's offices laid down clearly excessive requirements for suffi- 
cient grounds for suspicion. In view of negligibility, expert opinions were often not obtained and the proceedings were terminated in accordance with section 153 StPO.

According to the experts, the prosecution of self-doping could be improved by changes at an institutional level and in procedural law - in particular by establishing more specialised public prosecutor's offices and by introducing a leniency (see below II., 2.). In order to punish infringements of the ban on self-doping more effectively, the will of those involved in sport and the enforcement of regular testing for doping are crucial. In addition, the experts consider a moderate revision of sections 3 and 4 to be necessary in order to define the scope of the ban on self-doping more clearly and to eliminate existing ambiguities, especially concerning the determination of addressees contained in section 4 (7) (see II.1. below).

Overall, the recommendations should allow for a refocusing of the practical implementation of the AntiDopG: Investigations into violations of the doping ban in a competition of organised sport should become more central -rather than continuing to devote the majority of resources of the customs authorities, the police and the public prosecutor's offices to investigations against bodybuilders and other self-users in recreational sport. The proposals concerning section 2 serve the same purpose.

2. Unlawful handling of doping substances and unlawful application of doping methods (section 2)

The main focus of the investigations under the AntiDopG lies on the violations of the prohibitions pursuant to section 2. Self-users - primarily from the field of bodybuilding -represent the majority of the accused. Due to the fact that they do not participate in competitions where sporting and competition rules would need to be respected, fairness and equal chances or the integrity of sport cannot be adversely affected by the use of doping substances. The provision thereby primarily protects recreational athletes who are using doping substances from self-damaging acts. This is largely due to the fact that - according to the respondents - the DmMV lays down comparatively low thresholds for a significant quantity, so that the bodybuilders also who are using doping substances for themselves and not for the purpose of trafficking are prosecuted for the purchase and possession of doping substances. A large amount of the investigative resources taken up by the AntiDopG is therefore used to prosecute recreational athletes 
who are using doping substances regardless of any competitions and who are mostly harming themselves.

Investigations against people who traffic in doping substances on a large scale or manufacture doping substances are the exception. How far legal practice has currently widened the scope of the prohibition under section 2 is impressively demonstrated by a preliminary investigation that has been conducted against an overweight defendant who had ordered a dietary product that contained an ingredient pursuant to the AntiDopG.

"An obviously overweight man had ordered doping substances, or at least substances listed in the DmMV. He told the police he was only looking for a weight loss product. But training in order to reduce weight that is supported by the use of doping substances can also be regarded as doping in sport. But things get more complicated when he says that he never even wanted to do sport in the first place." (StA 5)

With such a broad understanding of the offence, the criminal liability thus depends on whether the accused wants to achieve weight loss (also) through exercising or solely by taking the medicinal product. If he does exercise, he could fall within the scope of section 2; if he refrains from doing so and only takes the medicinal product, he clearly goes unpunished. The different handling of these variations cannot be justified by the purpose of the Act - health protection and protection of the integrity of sport. Even more: the discrepancy confirms the doubts whether the broad interpretation of the prohibition of purchase and possession would be compatible with the objectives of the AntiDopG and the purpose of section 2 (3). For the investigative resources to be more focused on punishable acts, a proposal that considers the objectives referred to in section 1 and implements the legislative purpose pursued by section 2 (3) more consistently is made below (see II.2.b. below); an amendment of section 2 (3) or even a redesign of the legitimation model of section 2 is not necessary.

\section{Specific recommendations of the experts}

1. Substantive amendments

a) Self-doping

The evaluation has shown that the restriction of the group of perpetrators contained in section 4 (7) has proven to be the main substantive issue con- 
cerning the ban on self-doping. In particular, the determination of "significant revenue" causes difficulties in practice and often is the main reason for the termination of the proceeding. These issues can be addressed in two ways: (1) By specifying section 4 (7) or (2) by removing the restriction.

(1) The widespread uncertainties as to what constitutes significant revenue are not that untypical for a new law. However, given the rarity of criminal convictions, it might take some time for these issues to be clarified by the highest court. Since athletes apparently assume a much higher threshold than the public prosecutors do and are therefore not aware of the actual risks of criminal liability, a clarification of the provision - if one wants to maintain such a restriction of offenders - seems reasonable. Where the legislator wants to set the threshold for significant revenue is a question of legal policy. In accordance with current practice, it would make sense to assume significant revenue if it exceeds the marginal earnings threshold referred to in section 8 (1) of Volume IV of the Social Insurance Code after the expenses incurred for the sporting activity have been deducted.

Within the context of a reform of section 4 (7), it would also be advisable to incorporate the regulation on the group of perpetrators into section 3. The prerequisites for being a perpetrator represents a considerable restriction of criminal liability, which -including for reasons of comprehensibility - should already be contained in the prohibition itself and not only in the provision on sanctions.

Whether the prerequisites for being a perpetrator will be formulated as an element or as a solely objective condition of criminal liability is within the discretion of the legislator; however, there is little reason to decide for a solely objective condition of criminal liability.

(2) However, a moderate reform of section 4 (7) in accordance with the principles outlined above cannot solve the fundamental problems related to the restriction of the circle of addressees. A specification of "significant revenue" does indeed provide clarity for the criterion. However, it does not change the fact that the investigations are complex: The public prosecutor's offices would have to reconstruct the accused's income from sport and his or her expenses for sport. This encourages the termination of proceedings due to considerations of practical expediency at an early stage.

The restriction of perpetrators was introduced by the legislator in order to exempt "mere recreational athletes" from criminal liability. As explained above, however, section 4 (7) defines the circle of addressees much narrower (see in detail above B. I. 2.c.). Competitive athletes who have not (yet) been included in a Registered Testing Pool (such as athletes from the 
B-squad) and who - which is usual for a large number of sports - do not generate significant revenue are also exempted from criminal liability. However, the purpose of the AntiDopG (especially of section 3) to protect fundamental values of sport from being jeopardised by doping will be affected regardless of whether the participant in a sport competition is included in a Registered Testing Pool or is about to be included. ${ }^{144}$ Participation in a competition of organised sport where sport-specific rules need to be respected is crucial for the legally protected right to "integrity of sport". However, it is not relevant whether the athletes are included in a Registered Testing Pool or whether they generate any revenue from these competitions. ${ }^{145}$ In the public perception, the focus will also lie on the sporting event only; the fact whether or not the athlete is covered by the provision will not become relevant for the undermining of the trust in fairness of sport.

In addition, the restriction of perpetrators contradicts fundamental equity considerations and only provides insufficient protection for honest athletes. The regulation in section 4 (7) leads to a different treatment of athletes who compete against each other in the same competition. It would probably be very difficult to communicate to an athlete included in a Registered Testing Pool that he or she is subject to the AntiDopG, whereas his or her competitor who is also fighting for victory at the exact same event will not be covered by the AntiDopG.

Nor is it necessary to limit the scope of the group of perpetrators just in order to respect the principle of proportionality. ${ }^{146}$ The requirement of (planned) participation in a "competition of organised sport" constitutes a sufficiently high hurdle in order to exempt mere recreational sport from criminal liability. According to the explanatory memorandum to the Act, "sport competitions of top-level and competitive sport" where recreational athletes do not participate shall be covered. Private tournaments are therefore exempted from the scope of the prohibition. ${ }^{147}$ The fact that recreational athletes take part in competitions of organised sport is clearly an

144 See also MüKo-StGB/Freund, 3rd ed. 2018, AntiDopG, S\$ 1-4 Rn.112; Brill, SpuRt 2015, 153 et seq.

145 Particularly with regard to the legally protected right, it is not convincing that the generation of significant revenue should be decisive for criminal liability: Monetary aspects cannot decide over the question whether the integrity of sport has been harmed.

146 BT-Drs. 18/4898, p. 31.

147 BT-Drs. 18/4898, p. 27. 


\section{Final opinion of the experts}

exception that is mainly limited to bigger running events. Doping should probably not have any practical significance for such groups of persons. ${ }^{148}$

The experts therefore recommend to remove the restriction of the group of perpetrators contained in section 4 (7).

It seems advisable to emphasise the restrictive potential of the element "competition of organised sport" in terms of criminal liability within the explanatory memorandum to the Act. The evaluation of the files and the expert interviews both indicate that (too) little importance has been attached to this element so far. In this respect, it could also be pointed out that participation in competitions for bodybuilding is not covered by sections 3 and 4. Although the training process may constitute a sporting activity, the subsequent competition is not purely sport-related, but rather a presentation of their body shaping. ${ }^{149}$

If the legislator wishes to maintain an explicit restriction of the circle of addressees, it would be advisable to choose a demarcation criterion that precisely defines the intended scope of application. Here, one could consider formulating an exemption from criminal liability for "recreational athletes". The required legal definition could particularly be developed in cooperation with the NADA, who is currently working on a corresponding definition for the national Anti-Doping Code. Here, it should be considered that the prerequisites are practical and may not result in a major investigative effort.

b) Unlawful handling of doping substances (section 2)

aa) In order to concentrate the scarce resources of the public prosecutor's offices on cases of greater importance and, at the same time, in order not to overburden recreational athletes who were using doping substances with investigations affecting their fundamental rights, two options are possible:

(1) First of all, in accordance with an opinion in literature, one could demand a competitive aspect for the prohibitions contained in section 2 to

148 In case the investigating authorities actually become aware of self-doping in this field, it can be assumed - also in view of the current practice - that such proceedings will be terminated.

149 See also Brill, SpuRt 2015, 153, 154; MüKo-StGB/Freund, 3rd ed. 2018, AntiDopG, $\mathbb{S} \mathbb{S} 1-4$ Rn. 39. 
be applied. ${ }^{150}$ This would bring the prohibitions in section 2 closer together with those in section 3 and section 2 could thereby not only be justified by the objective of health protection but also by the objective of integrity of sport. However, such an interpretation would lead to the fact that the health protection that has been declared as the primary purpose of section 2 by the legislator becomes less important. Even if the competitive aspect was only limited to section 2 (3), the purchase and possession of significant quantities of doping substances that are clearly not only for personal use would then go unpunished. This would dogmatically and practically undermine the legislative decision to criminalise purchase and possession as precursors of trafficking.

(2) It is therefore preferable to find another way to interpret section 2 (3) in a more appropriate manner bearing in mind the legislative objective and to achieve a more target-oriented use of investigative resources. According to the explanatory memorandum to the Act, "experience has shown [that the purchase and possession of significant quantities of doping substances as referred to in section 2 (3)] are precursors of trafficking in these substances" ${ }^{151}$ Due to the fact that these substances, when used for the purpose of doping, have a particularly adverse effect on the health of athletes, "the risk of passing on should already be effectively prevented. Therefore, any actions that typically only serve to prepare for the passing on are already prohibited." 152

However, the evaluation has shown that proceedings are also conducted against the accused where the quantities found are so small that the purchase or possession - even in the opinion of the investigators - cannot be regarded as a precursor of trafficking or the passing on to third parties. In the interviews, the respondents confirmed that the proceedings were mainly directed against "self-users". The DmMV whose thresholds are set so low that they are exceeded in almost every case so that people without the intention of passing on or trafficking are also included was referred to as the main reason for this by the interviewees.

This is compounded by the fact that in legal practice, several - in itself insignificant- quantities of different substances are often added together in order to exceed the threshold for a significant quantity. In view of the legislative objective and the legitimation model that has been chosen by the

150 MüKo-StGB/Freund, 3rd ed. 2018, AntiDopG, $\mathbb{S}$ 1-4 Rn. 23, 64.

151 BT-Drs. 18/4898, p. 25; MüKo-StGB/Freund, 3rd ed. 2018, AntiDopG, $\$ \$ 1$ 1-4 Rn. 58.

152 See BT-Drs. 126/15, p. 24 f.; BT-Drs. 18/4898, p. 25. 
legislator, this practice is extremely questionable. If, for example, two opened ampoules containing different doping substances are found, the passing on of the remaining substances for the purpose of trafficking would almost be inconceivable; nevertheless, proceedings were conducted in these cases.

Finally, the evaluation has shown that investigations are increasingly directed against people who have purchased so-called anti-aging and lifestyle drugs for their own use without the intention of doping. This is another proof that there is no reasonable demarcation of non-punishable self-use of insignificant quantities currently.

The experts therefore recommend to further align the DmMV with the legislative objectives of section 2 (3) and to lay down thresholds for quantities where an intention of trafficking can be suggested reasonably certain.

The experts also recommend that the DmMV clarifies that the addition of insignificant quantities it is not permitted.

bb) The offences by negligence under section 4 (6) have not acquired any practical significance. This may also be due to the fact that negligent manufacturing, trafficking or possession of doping substances "for the purpose of doping human beings" is hardly conceivable. In practice, proceedings for negligent conduct are very rare and are usually terminated. This also indicates that the public prosecutor's offices consider the wrongfulness and guilt - if given - to be minor. The "health endangering" nature of the provision as referred to by the legislator does not call for a criminalisation. It would rather be necessary to explain why the society needs to be protected from a negligent violation of the prohibitions under section 4 (1) nos. 1 to 3. Justification can hardly be given. The evaluation has shown that there is no need for such protection. Because unlike those offences that were referred to as an example in the explanatory memorandum to the Act (negligent bodily harm, negligent arson and negligent driving under influence of drink or drugs), section 4 (6) plays no major role in practice. The investigative effort and their considerable impact on the accused's fundamental rights are therefore disproportionate to their benefit and especially to the offender's guilt.

Due to the fact that there is no compelling punishable cause for the offences by negligence under section 4 (6) and that the combination of negligent elements and special intentions is hard to conceive and that there is 
no practical need for a "catch-all element", the experts give the following recommendation:

The experts recommend deleting section 4 (6).

2. General procedural and institutional changes

a) Leniency and whistleblower systems

Experienced investigators explained that the rather minor practical significance of the ban on self-doping in criminal proceedings and the comparatively few proceedings against competitive athletes can be traced back to the fact that "we just do not receive such cases" (StA 3). This is also due to the fact that doping remains secret in this field and that the athletes or their environment have little incentive to reveal knowledge of any criminal offences - especially if this could lead to criminal investigations against themselves.

For this reason, the experts recommend that the sports associations should give their athletes much more information about the existence and functioning of whistleblower systems of the NADA and the WADA.

On the other hand, the experts recommend that a leniency that is adapted to the particularities of doping in sport should be introduced to the AntiDopG.

b) Specialised public prosecutor's offices and specialised courts

The evaluation has shown that the investigation of infringements of the AntiDopG requires a range of legal, medical and criminalistic expertise. This can - according to the findings of the evaluation - predominantly be found in specialised public prosecutor's offices, while other public prosecutor's offices without such specialisation often face problems when implementing the AntiDopG.

For this reason, the experts recommend that all federal States should follow the example of the States of Baden-Wuerttemberg, Bavaria and Rhineland-Palatinate and establish specialised public prosecutor's offices. 
C. Final opinion of the experts

However, the evaluation did not find a comparable need for the establishment of specialised courts. 


\section{Annex: Guideline for public prosecutors}

\section{A. Key data}

1) For how long have you been working in a department that deals with cases of doping?

2) Approximately how many cases of doping have you been concerned with?

\section{B. Phenomenon / Cases}

\begin{tabular}{|l|l|l|}
\hline What do typical cases of doping that you are concerned with look like? \\
\hline $\begin{array}{l}\text { What do we want to } \\
\text { know? }\end{array}$ & Further questions & Notes \\
\hline & $\begin{array}{l}\text { Which sports / sports federation } \\
\text { are mostly concerned? }\end{array}$ & \\
$-\begin{array}{l}\text { Which doping substances / do- } \\
\text { ping methods are mostly used? }\end{array}$ & $-\begin{array}{l}\text { How have the cases of doping } \\
\text { been detected? Could you give an } \\
\text { illustrative example? }\end{array}$ \\
$-\begin{array}{l}\text { What is the main focus: backers } \\
\text { or self-users? Popular sport or } \\
\text { competitive sport? }\end{array}$ & $\begin{array}{l}\text { Are there also any cases of orga- } \\
\text { nised crime? Rather in the field } \\
\text { of backers or in laboratories? } \\
\text { Could you give an illustrative } \\
\text { example? }\end{array}$ \\
\hline
\end{tabular}




\section{Investigations}

\begin{tabular}{|l|l|l|}
\hline $\begin{array}{l}\text { Part 1: Initial suspicion } \\
\text { What is your initial suspicion } \\
-\quad \text { of unlawful handling of doping substances or unlawful application of } \\
\text { doping methods (section 2) } \\
- \text { of self-doping (section 3) } \\
\text { usually based on? }\end{array}$ & Notes \\
\hline \begin{tabular}{l|l|l|} 
What do we want to \\
know?
\end{tabular} & Further questions \\
\hline $\begin{array}{l}\text { (ex officio, report of an } \\
\text { offence made by whist- } \\
\text { leblowers, sports federati- } \\
\text { ons or the NADA) }\end{array}$ & $\begin{array}{l}\text { Do other proceedings (e.g. } \\
\text { against underground laboratories) } \\
\text { often provide grounds for further } \\
\text { proceedings? Could you give an il- } \\
\text { lustrative example? }\end{array}$ & \\
\hline Other proceedings & $\begin{array}{l}\text { Do proceedings for violations of } \\
\text { section 2 often provide grounds } \\
\text { for proceedings for self-doping } \\
\text { pursuant to section 3? }\end{array}$ & \\
\hline NADA & $-\begin{array}{l}\text { How much does your investiga- } \\
\text { tive work rely on information } \\
\text { provided by the NADA? } \\
\text { Have you relied on any docu- } \\
\text { ments from proceedings under } \\
\text { the Corporate Liability Act for } \\
\text { your investigations? }\end{array}$ & \\
\hline Evaluation and & $\begin{array}{l}\text { Would you consider the existing } \\
\text { Rays of obtaining knowledge suf- } \\
\text { ficient? Where appropriate: What } \\
\text { could be done to improve the ob- } \\
\text { tainment of knowledge of the } \\
\text { public prosecutor's offices? }\end{array}$ & \\
\hline
\end{tabular}




\begin{tabular}{|c|c|c|}
\hline \multicolumn{3}{|c|}{$\begin{array}{l}\text { Part 2: Investigation measures and means of compulsion / evidentia- } \\
\text { ry problems } \\
\text { What kind of investigation measures and means of compulsion do you } \\
\text { take and to what effect? (differences between sections } 2 \text { and 3?) }\end{array}$} \\
\hline $\begin{array}{l}\text { What do we want to } \\
\text { know? }\end{array}$ & Further questions & Notes \\
\hline Applied measures & $\begin{array}{l}\text { What kind of investigation } \\
\text { measures do you usually take in } \\
\text { order to investigate cases of dop- } \\
\text { ing? (differences between sections } \\
2 \text { and 3?) }\end{array}$ & \\
\hline $\begin{array}{l}\text { Telecommunications } \\
\text { surveillance }\end{array}$ & $\begin{array}{l}- \text { Has the admissibility of telecom- } \\
\text { munications surveillance im- } \\
\text { proved your means of investigat- } \\
\text { ing? }\end{array}$ & \\
\hline Leniency & $\begin{array}{l}- \text { How important is the already ap- } \\
\text { plicable leniency that is restric- } \\
\text { ted to cases of doping committed } \\
\text { on a commercial basis or as a } \\
\text { gang in accordance with section } \\
2(1) \text { and }(2)^{*} \text { ? } \\
-\quad \text { Would you consider the exten- } \\
\text { sion of the scope of the leniency } \\
\text { necessary for your work? }\end{array}$ & \\
\hline $\begin{array}{l}\text { Evaluation and } \\
\text { Recommendations } \\
\end{array}$ & $\begin{array}{l}\text { - What are new means of investi- } \\
\text { gating under the AntiDopG? } \\
\text { Were they useful and sufficient? } \\
\text { What additional legal authori- } \\
\text { ties would be useful to effectively } \\
\text { investigate cases of doping? }\end{array}$ & \\
\hline Evidentiary problems & $\begin{array}{l}\text { - What are the main difficulties } \\
\text { when providing evidence? }\end{array}$ & \\
\hline $\begin{array}{l}\text { Specialised public } \\
\text { prosecutor's office/ } \\
\text { specialised court }\end{array}$ & $\begin{array}{l}\text { What is the advantage of a spe- } \\
\text { cialised public prosecutor's of- } \\
\text { fice? Would you consider the es- }\end{array}$ & \\
\hline
\end{tabular}

* Section 46b StGB: Prerequisite is a minimum penalty. This is only the case with gangs/commercial activities. 


\begin{tabular}{|l|l|} 
tablishment of specialised pu- \\
blic prosecutor's offices useful? \\
$-\begin{array}{l}\text { Would you consider the estab- } \\
\text { lishment of specialised courts } \\
\text { useful? }\end{array}$
\end{tabular} \mid

\section{Part 3: Conclusion of proceedings}

- How are the proceedings under section 2 usually concluded? Are there any particularities compared to other offences?

- How are the proceedings under section 3 usually concluded? Are there any particularities compared to other offences?

\begin{tabular}{|c|c|c|}
\hline \multicolumn{3}{|c|}{$\begin{array}{l}\text { Part 4: Information-sharing with the NADA } \\
\text { How important is the information-sharing pursuant to section } 8 \text { Anti- } \\
\text { DopG? }\end{array}$} \\
\hline $\begin{array}{l}\text { What do we want to } \\
\text { know? }\end{array}$ & Further questions & Notes \\
\hline Practical procedures & $\begin{array}{l}- \text { How does the cooperation work } \\
\text { in practice? } \\
-\quad \text { How often do you submit infor- } \\
\text { mation and for what reasons? (in } \\
\text { general or in individual cases on- } \\
\text { ly - according to which criteria?) }\end{array}$ & \\
\hline NADA's response & $\begin{array}{l}\text { Were there any consequences on } \\
\text { the part of the NADA in those } \\
\text { cases where you have provided } \\
\text { the information? Is this the usual } \\
\text { case? }\end{array}$ & \\
\hline $\begin{array}{l}\text { Evaluation and } \\
\text { recommendations }\end{array}$ & $\begin{array}{l}\text { - How would you rate the relevant } \\
\text { amendments? Would you have } \\
\text { any suggestions for improvement } \\
\text { or do you consider the provision } \\
\text { sufficient? }\end{array}$ & \\
\hline
\end{tabular}




\section{Legal issues}






\begin{tabular}{|c|c|}
\hline & $\begin{array}{l}\text { gnificant quantities" appro- } \\
\text { priate? }\end{array}$ \\
\hline \begin{tabular}{|l} 
Modification of \\
especially serious cases \\
into penalty enhancing \\
$\underline{\text { offences }}$
\end{tabular} & $\begin{array}{l}\text { - Especially serious cases were } \\
\text { modified into penalty enhan- } \\
\text { cing offences due to the new ver- } \\
\text { sion. Did this lead to any changes } \\
\text { in practice? (serious criminal of- } \\
\text { fence/ termination no longer pos- } \\
\text { sible) }\end{array}$ \\
\hline $\begin{array}{l}\text { Possible offences that } \\
\text { include actions prior to } \\
\text { money laundering }\end{array}$ & $\begin{array}{l}\text { - Has anything changed in practice } \\
\text { due to the fact that the criminal } \\
\text { acts are now possible offences } \\
\text { that include action prior to mo- } \\
\text { ney laundering? }\end{array}$ \\
\hline $\begin{array}{l}\text { Explicit inclusion of the } \\
\text { unlawful application of } \\
\underline{\text { doping methods }}\end{array}$ & $\begin{array}{l}\text { - The law now also covers the un- } \\
\text { lawful application of doping me- } \\
\text { thods. } \\
\text { - How would you evaluate this } \\
\text { legislative amendment? } \\
\text { - Could you give an illustra- } \\
\text { tive case example where the } \\
\text { legislative amendment has } \\
\text { made a legal difference, e.g. } \\
\text { caused a different outcome } \\
\text { of the proceeding? }\end{array}$ \\
\hline $\begin{array}{l}\text { Evaluation and } \\
\text { recommendations }\end{array}$ & $\begin{array}{l}\text { Do you consider the offence suc- } \\
\text { cessful overall? What would you } \\
\text { change if necessary? }\end{array}$ \\
\hline
\end{tabular}




\begin{tabular}{|c|c|c|}
\hline \multicolumn{3}{|c|}{$\begin{array}{l}\text { Part 2: Legal issues arising under section } 3 \text { AntiDopG } \\
\text { Have any legal issues occurred when applying the new law on self-dop- } \\
\text { ing? Which ones? }\end{array}$} \\
\hline $\begin{array}{l}\text { What do we want to } \\
\text { know? }\end{array}$ & Further questions & Notes \\
\hline $\begin{array}{l}\text { Self-doping: General } \\
\text { assessment }\end{array}$ & $\begin{array}{l}\text { Are the offences concerning self- } \\
\text { doping sufficiently precise and } \\
\text { practical? }\end{array}$ & $\begin{array}{l}\text { Prob- } \\
\text { lems re- } \\
\text { garding } \\
\text { competi- } \\
\text { tive ath- } \\
\text { letes? } \\
\text { Rev- } \\
\text { enue? }\end{array}$ \\
\hline circle of addressees & $\begin{array}{l}\text { Has the circle of addressees con- } \\
\text { tained in section } 4 \text { (7) proven it- } \\
\text { self in practice? } \\
\text { - How do you define the term } \\
\text { "significant revenue"? }\end{array}$ & \\
\hline Objective elements & $\begin{array}{l}\text { Which one of the objective ele- } \\
\text { ments causes legal problems } \\
\text { more frequently (circle of ad- } \\
\text { dressees, lack of medical indica- } \\
\text { tion)? Could you give an illustra- } \\
\text { tive example? }\end{array}$ & \\
\hline Subjective elements & $\begin{array}{l}\text { - Do the subjective requirements } \\
\text { cause any particular difficulties? } \\
\text { (for example: awareness of the } \\
\text { circle of addressees, lack of medi- } \\
\text { cal indication, etc.) }\end{array}$ & \\
\hline $\begin{array}{l}\text { Criminal liability for } \\
\text { purchase and possession } \\
\underline{\text { without quantitative }} \\
\underline{\text { restrictions }}\end{array}$ & $\begin{array}{l}\text { - Has the criminalisation of the } \\
\text { purchase or possession of insi- } \\
\text { gnificant quantities for the pur- } \\
\text { pose of self-doping proven itself } \\
\text { in practice? }\end{array}$ & \\
\hline $\begin{array}{l}\text { Lack of criminal } \\
\text { liability for attempted } \\
\text { purchase or possession }\end{array}$ & $\begin{array}{l}\text { Is the lack of criminal liability } \\
\text { for attempt appropriate or do } \\
\text { you regard it as a privilege for } \\
\text { certain athletes? }\end{array}$ & \\
\hline
\end{tabular}




\begin{tabular}{|c|c|}
\hline $\begin{array}{l}\text { Evaluation and } \\
\text { recommendations }\end{array}$ & $\begin{array}{l}\text { - Do you consider the offence suc- } \\
\text { cessful overall? What would you } \\
\text { change if necessary? }\end{array}$ \\
\hline
\end{tabular}

\begin{tabular}{|c|c|c|}
\hline \multicolumn{3}{|c|}{$\begin{array}{l}\text { Part 3: Sentencing range and limitation period } \\
\text { How would you evaluate the provisions on the sentencing ranges and } \\
\text { limitation periods? }\end{array}$} \\
\hline $\begin{array}{l}\text { What do we want to } \\
\text { know? }\end{array}$ & Further questions & Notes \\
\hline Sentencing ranges & $\begin{array}{l}\text { Do you consider the sentencing } \\
\text { ranges consistent and appropri- } \\
\text { ate? Do you consider the privileged } \\
\text { treatment of athletes in terms of sen- } \\
\text { tencing range for the criminal liabi- } \\
\text { lity for possession to be appropriate? }\end{array}$ & \\
\hline Limitation period & $\begin{array}{l}\text { Are the limitation periods rea- } \\
\text { sonably long? }\end{array}$ & \\
\hline $\begin{array}{l}\text { Evaluation and } \\
\text { recommendations }\end{array}$ & $\begin{array}{l}\text { Do you consider the provisions of } \\
\text { the AntiDopG successful in these } \\
\text { points? What changes would you } \\
\text { recommend if necessary? }\end{array}$ & \\
\hline
\end{tabular}

\section{E. Court proceedings}

\section{Court proceedings}

What experiences have you made in the subsequent court proceedings?

\begin{tabular}{|l|l|l|}
\hline $\begin{array}{l}\text { What do we want to } \\
\text { know? }\end{array}$ & Further questions & Notes \\
\hline Duration & $\begin{array}{l}\text { Approximately how long do } \\
\text { court proceedings take? Is there } \\
\text { anything that can cause delays? }\end{array}$ & \\
\hline Evidentiary problems & $\begin{array}{l}\text { What were the challenges in ta- } \\
\text { king evidence at the main hear- } \\
\text { ing? }\end{array}$ & \\
\hline
\end{tabular}




\begin{tabular}{|l|l|l|}
\hline Negotiated agreements & $-\begin{array}{l}\text { Were there any negotiated agree- } \\
\text { ments? } \\
-\end{array}$ & $\begin{array}{l}\text { What were the prosecution's mo- } \\
\text { tives for the negotiated agree- } \\
\text { ment? } \\
\text { Who took the initiative to reach a } \\
\text { negotiated agreement? }\end{array}$ \\
\hline
\end{tabular}

\section{F. General assessments}

\section{Part 1: Opinion on the criminal liability for self-doping}

In the literature, the criminal liability for self-doping is sometimes viewed critically. For example, it is argued that the AntiDopG would lead to a "massive over-criminalisation of popular sport" or would use too many undefined legal concepts. Could you comment on this view?

\begin{tabular}{|l|l|l|}
\hline $\begin{array}{l}\text { What do we want to } \\
\text { know? }\end{array}$ & Further questions & Notes \\
\hline Opinion & $\begin{array}{l}\text { Do you have the impression that } \\
\text { the criminal prosecution of un- } \\
\text { lawful handling of doping sub- } \\
\text { stances or unlawful application } \\
\text { of doping methods (1) is general- } \\
\text { ly useful and (2) that you as a } \\
\text { public prosecutor can identify } \\
\text { with the objective? }\end{array}$ & \\
\hline
\end{tabular}

\section{Part 2: General assessment of the law}

How would you as a practitioner evaluate the AntiDopG? Would you consider the provisions "successful" overall?

\begin{tabular}{|l|l|l|}
\hline $\begin{array}{l}\text { What do we want to } \\
\text { know? }\end{array}$ & Further questions & Notes \\
\hline Section 2 & $\begin{array}{l}\text {... with regard to section 2 Anti- } \\
\text { DopG? }\end{array}$ & \\
\hline Section 3 & $\begin{array}{l}\text {... with regard to section 3 Anti- } \\
\text { DopG? }\end{array}$ & \\
\hline
\end{tabular}




\section{Part 3: Concrete reform proposals}

In your opinion, what are the most urgent areas of improvement on a factual, procedural or practical level?

\begin{tabular}{|l|l|l|}
\hline $\begin{array}{l}\text { What do we want to } \\
\text { know? }\end{array}$ & Further questions & Notes \\
\hline Factual level & & \\
\hline Procedural level & & \\
\hline Practical level & & \\
\hline (institutionally etc.) & & \\
\hline
\end{tabular}

Is there anything else that you think would be relevant to our research project that has not yet been addressed in this conversation? 


\section{Literaturverzeichnis/Bibliography}

\section{Literatur/Literature}

Armenat, Christin: Diskussionsbericht, in: Hoven, Elisa/Kubiciel, Michael (Hrsg.), Korruption im Sport, Baden-Baden 2018, S. 139-146.

Bogner, Alexander/Littig, Beate/Menz, Wolfgang: Interviews mit Experten, Wiesbaden 2014.

Brill, Sebastian: Das neue „Anti-Doping-Gesetz“ und die ungerechtfertigte Privilegierung von Bodybuildingwettkämpfen, SpuRt 2015, S. 153-155.

Cherkeh, Rainer: Stärkung des Hinweisgeberschutzes durch Kronzeugenregelung im AntiDopG, Zeitschrift für Sportrecht (SpuRt) 2019, S. 167- 169.

Eisenberg, Ulrich/Kölbel, Ralf: Kriminologie, 7. Aufl., Tübingen 2017.

Eising, Felix: Die Strafbarkeit des Eigendopings, Münster 2018.

Erbs, Georg/Kohlhaas, Max/ Häberle, Peter (Hrsg.): Kommentar zu Strafrechtlichen Nebengesetzen, 232. Ergänzungslieferung August München 2020.

Fiedler, Adrian: Das Doping minderjähriger Sportler, Berlin 2013.

Finken, Pia: Die neue Anti-Doping-Gesetzgebung: Gesetz zur Bekämpfung von Doping im Sport, Pharmarecht (PharmR) 2016, S. 445-449.

Freund, Georg: Verfassungswidrige Dopingstrafbarkeit nach $₫ 95$ Abs. 1 Nr. 2a AMG - Ein Beitrag zum Gesetzlichkeitsgrundsatz (Art. 103 Abs. 2 GG), in: Bannenberg, Britta (Hrsg.), Über allem: Menschlichkeit. Festschrift für Dieter Rössner, Baden-Baden 2015, S. 579-598.

Graf, Jürgen Peter/Jäger, Markus/Wittig, Petra (Hrsg.): Kommentar zum Wirtschafts- und Steuerstrafrecht, 2. Aufl., München 2017.

Hauptmann, Markus/Klarmann, Max: Whistleblower und Kronzeugen: Bedeutung und Regelungsbedarf im Interesse einer effizienteren Aufdeckung von Dopingverstößen im Sport, Zeitschrift für Sportrecht (SpuRt) 2019, S. 190-197.

Heger, Martin: Die Strafbarkeit von Doping nach dem Anti-Doping-Gesetz (ADG), Zeitschrift für Medizinstrafrecht (medstra), 2017, S. 205-216.

Heger, Martin: Strafrechtliche Besonderheiten im Umgang mit minderjährigen Leistungssportlern, in: Kauerhof, Rico/Nagel, Sven/Zebisch, Mirko (Hrsg.), Olympische Jugendspiele: Chance oder Gefahr? Dokumentation des 3. Leipziger Sportrechtstages 2009, Leipzig 2010, S. 25-42.

Helfferich, Cornelia: Leitfaden- und Experteninterviews, in: Baur, Nina/Blasius, Jörg (Hrsg.), Handbuch Methoden der empirischen Sozialforschung, 4. Aufl., Wiesbaden 2014, S. 559-574. 
Jahn, Matthias: Wir haben alles. Außer Strafverfahren. Erste Praxiserfahrungen mit der Rundumstrafbarkeit nach dem Anti-Doping-Gesetz in Deutschland, in: Hoven, Elisa/Kubiciel, Michael (Hrsg.), Korruption im Sport, Baden-Baden 2018, S. 117-124.

Joecks, Wolfgang/Miebach, Klaus (Hrsg.): Münchener Kommentar zum StGB, Bd. 6, 3. Aufl., München 2017.

Kaiser, Robert: Qualitative Experteninterviews. Konzeptionelle Grundlagen und praktische Durchführung, Wiesbaden 2014.

Kassner, Silke: Die Strafbarkeit des Dopings aus der Perspektive der Athleten, in: Hoven, Elisa/Kubiciel, Michael (Hrsg.), Korruption im Sport, Baden-Baden 2018, S. 135-138.

Körner, Hans/Patzak, Jörn/Volkmer, Mathias/Fabricius, Jochen (Hrsg.): Kommentar zum BtMG, 9. Aufl. 2019, München.

Kosfeld, Reihnhold/Eckey, Hans Friedrich/Türck, Matthias (Hrsg.): Deskriptive Statistik, 6. Aufl., Wiesbaden 2016.

Kreuzer, Arthur: Kriminalisierung des „Eigendoping“ von Sportlern? Desillusionierung und Umdenken in der Sportpolitik statt Ausweitung des Strafrechts, Zeitschrift für Rechtspolitik (ZRP) 2013, S. 181-184.

Kruse, Jan: Einführung in die Qualitative Interviewforschung, Freiburg 2011.

Kubiciel, Michael: Anmerkung zum LG Köln (Dopingnachweis bei geringer Steroidkonzentration), Zeitschrift für Sportrecht (SpuRt) 2020, S. 314-.316.

Kubiciel, Michael: Entwicklung des eSports und Schutz seiner Integrität, Zeitschrift für Rechtspolitik (ZRP) 2019, S. 200-203.

Kubiciel, Michael: Integrität des Sports - Konkretisierung eines Begriffs, Zeitschrift für Kriminalpolitik (KriPoZ) 2018, S. 29-31.

Lehner, Michael/Nolte, Martin/Putzke, Holm (Hrsg.): Nomos-Kommentar zum Anti-Doping-Gesetz, 2017, Baden-Baden.

Lorenz, Henning/Bade, Maximilian: Anm. zu OLG Köln, Beschl. v. 4.4.2019 - 2 Ws 122/19, Juristische Rundschau (JR) 2020, S. 322-327.

Momsen, Carsten: Integrität des Sports - Was sollen neue Tatbestände schützen?, Zeitschrift für Kriminalpolitik (KriPoZ) 2018, S. 21-28.

Peukert, Matthias: Das neue Anti-Doping-Gesetz - Ein erster kritischer Überblick aus strafrechtlicher Sicht, Zeitschrift für das Recht der Non Profit Organisationen (npoR) 2015, S. 95-101.

Rössler, Patrick/Geise, Stephanie: Standardisierte Inhaltsanalyse: Grundprinzipien, Einsatz und Anwendung, in: Möhring, Wiebke/Schlütz, Daniela (Hrsg.), Handbuch standardisierte Erhebungsverfahren in der Kommunikationswissenschaft, Berlin 2013, S. 269-288.

Samson-Baudisch, Marc: Der Missbrauch von anabol-androgenen Steroiden im freizeitorientierten Bodybuilding: eine empirische Untersuchung von hyperinkludierten Körpermodellierern, Hamburg 2014.

Steiner, Udo: Verfassungsfragen des Sports, Neue Juristische Wochenschrift (NJW) 1991, S. 2729-2736. 
Weber, Klaus (Hrsg.): Kommentar zum BtMG, 5. Aufl., München 2017.

\section{Sonstige Dokumente/Other documents}

Athleten Deutschland e.V.: Anhörung des Sportausschusses, 23.10.2019: Stellungnahme, Berlin 2019.

Deutscher Anwaltverein: Stellungnahme zur öffentlichen Anhörung des Sportausschusses im Deutschen Bundestag zum Thema „Änderungs- und Ergänzungsbedarfe im Anti-Doping-Gesetz“, Nr. 38/2019, Berlin 2019.

DOSB: Öffentliche Anhörung des Sportausschusses am 23. Oktober 2019 zum Thema „Änderungs- und Ergänzungsbedarfe im Anti-Doping-Gesetz (AntiDopG)“, Frankfurt a.M 2019.

DOSB: Stellungnahme Anti-Doping Gesetz durch die Athletenkommission, Frankfurt a.M 2015.

NADA: Öffentliche Anhörung des Sportausschusses des Deutschen Bundestages, Änderungs- und Ergänzungsbedarfe im Anti-Doping-Gesetz (AntiDopG), Bonn 2019.

NADA: Übersicht Risikogruppen, Bonn 2016, abrufbar unter: https:/www.nada.de /fileadmin/user_upload/nada/DKS/160729_UEbersicht_Risikogruppen.pdf (letzter Abruf: 26. Mai 2020). 
